\title{
Measuring E-Learning Readiness in the Forestry Research and Development Agency of Indonesia
}

\author{
By \\ Retisa Mutiaradevi
}

\begin{abstract}
A thesis
submitted to the Victoria University of Wellington in fulfilment of the requirements for the degree of Master of Commerce and Administration in Information Systems
\end{abstract}

Victoria University of Wellington 


\section{Abstract}

This research investigates organisational readiness for implementing organisational elearning systems (OES) in the Forestry Research and Development Agency (FORDA) of Indonesia. The study measures the level of organisational readiness for OES implementation at FORDA; and determines factors that need to be addressed in order to implement successful OES at FORDA, in a context with no prior OES knowledge. A mixed-methods approach was employed in this study. A cross-sectional survey was delivered in two forms: online and paper-based. The survey sample consisted of 288 employees of FORDA (policy makers, researchers, research assistants, and general employees). Statistical measures - reliability analysis, factor analysis, and multiple regression - were conducted using SPSS version 16.0. As a complementary method, 12 semi-structured interviews were conducted with 12 policy makers at FORDA.

A comprehensive organisational e-learning readiness instrument (COERI) was developed to assess the level of organisational readiness for OES from the perspective of all target respondents (policy makers, e-learning providers, facilitators, and learners). The study proposes twelve readiness dimensions: technological skills; positive online learning style; negative online learning style; equipment/infrastructure; attitude; human resources; environmental; positive cultural; negative cultural; financial affordability; financial availability; and financial possibility.

Overall, to implement successful OES at FORDA the results show that only five areas are ready but need a few improvements (equipment/infrastructure, attitude, environment, positive online culture, and financial availability); five areas are not ready of which three areas need some improvements (technological skills, positive online learning style, and human resources), and two areas need major improvements (financial affordability and financial possibility). Factors that affect organisational readiness and need to be developed further in order to implement successful OES are: personal characteristics (age, education, gender, and computer experience), Internet access, central support, finance, environment, face-to-face contact, human resources capacity, English proficiency, and prior e-learning knowledge. Further exploration is required to achieve a more reliable instrument and to improve the applicability of COERI to similar research contexts. 
Keywords: e-learning, online learning, e-learning readiness, e-learning readiness measurement, organisational e-learning systems (OES), e-learning knowledge, online training, developing countries 
'Truth is what stands the test of experience"

- Albert Enstein-

\section{Dedicated to}

my mother who nurtured my erly childhood knowledge.. and to

my daughter who will cary on her knowledge to the next generdion.. 


\section{Acknowledgements}

Completing an MCA thesis is challenging. During my study, I received support and help from many people.

I would like to express my sincere gratitude to my research supervisor Professor Pak Yoong for his support, encouragement, and patient supervision. My sincere thanks to Dr. Colin Jeffcoat for giving time and valuable suggestions to me. I also gratefully acknowledge Zaafri Husodo, Ph.D and Rahmadi Murwanto, MBA for sharing their knowledge on drawing a sample from a population for my survey. Appreciation is also given to Dr. Nokuthaba Sibanda for assisting me with the preparation and analysis of the quantitative data.

I would also like to thank my friends and peer reviewers, especially Nicole Braun, Silke Retzer, and Paul Atkinson for providing support, encouragement, and particularly for their valuable feedback. Special thanks also go to my proofreader Jackie Bell. My deepest gratitude goes to Jay Drew, Bridget Nankivell, Inge De Leeuw, and Tiso Ross for giving me all the necessary practical assistance during my studies at Victoria University of Wellington.

I would also like to express my special thanks to policy makers and colleagues at FORDA for taking part in the pilot test survey, providing feedback, support and encouragement, as well as participating in my research.

My greatest appreciation goes to my whole family, especially to my brave little girl, Sara, who always believes in me and gives me the courage and the strength to be all that I can be. My special gratitude also goes to my husband for his patience and effort in keeping things going after all that we have been through.

Last but not least, I would like to acknowledge that I am the only person responsible for errors in this thesis. 


\section{Table of Contents}

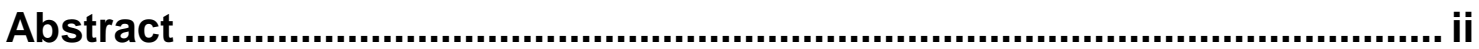

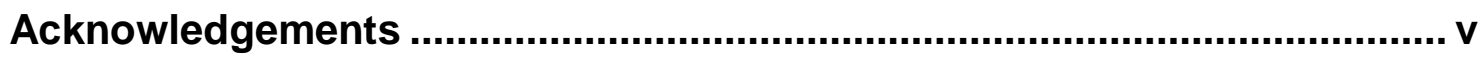

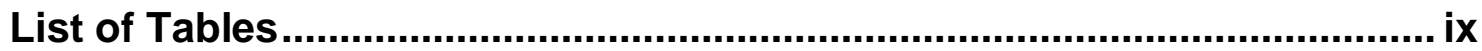

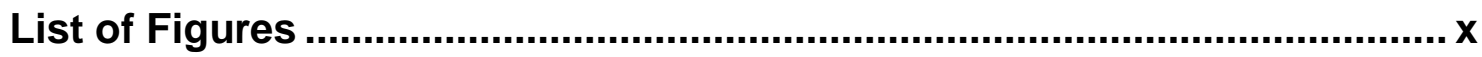

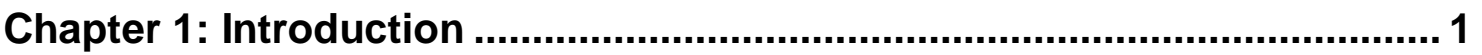

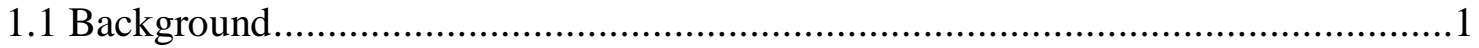

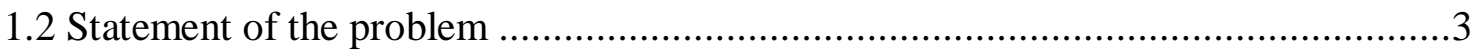

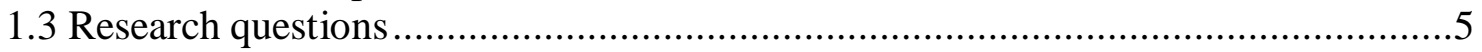

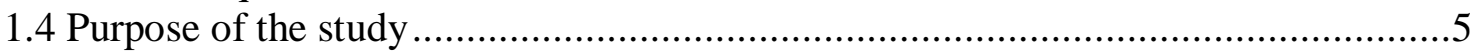

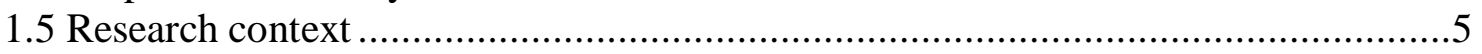

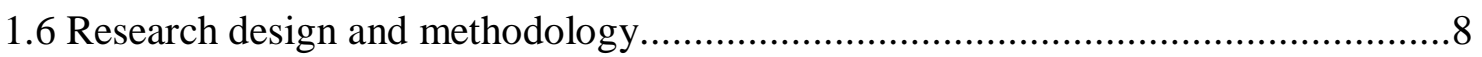

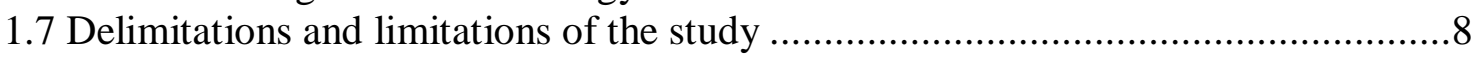

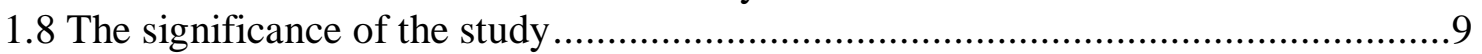

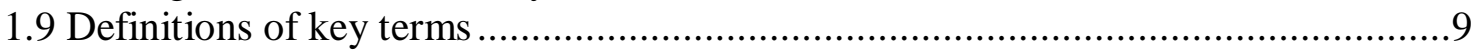

1.10 The structure of the research..........................................................................

Chapter 2: Literature Review .................................................................. 11

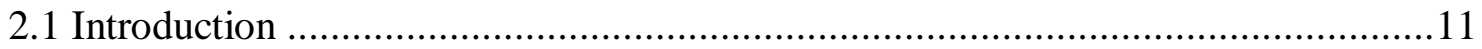

2.2 Justification for examining organisational readiness for OES ................................11

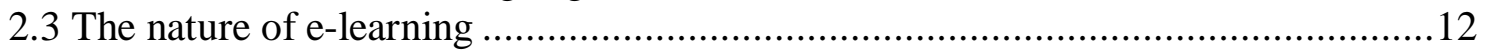

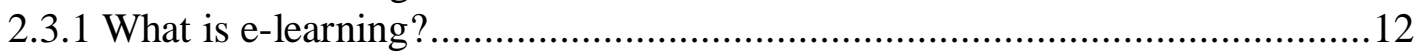

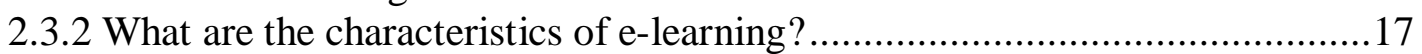

2.3.3 Why do we need organisational e-learning? ……………............................20

2.3.4 What are the downsides of e-learning implementation? ................................24

2.4 Critical success factors for developing and implementing OES .............................28

2.5 Assessing organisational readiness for OES ……………....................................

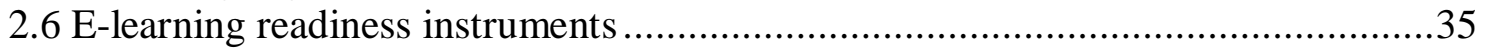

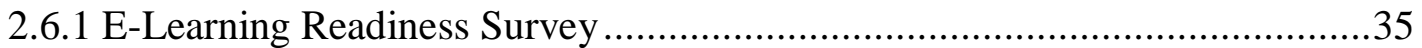

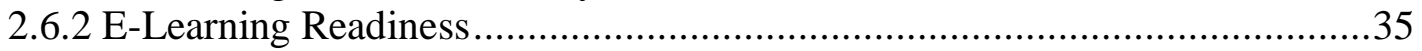

2.6.3 Tertiary Students' Readiness for Online Learning........................................36

2.6.4 Individual Readiness to Develop and Implement E-Learning ..........................36

2.6.5 E-Learning Readiness Self-Assessment .......................................................37

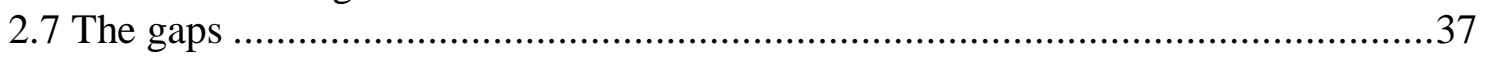

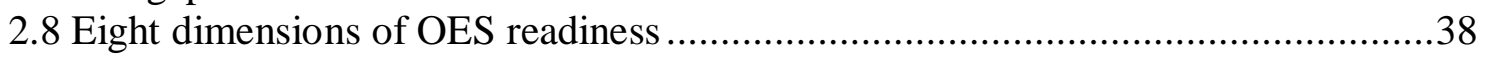

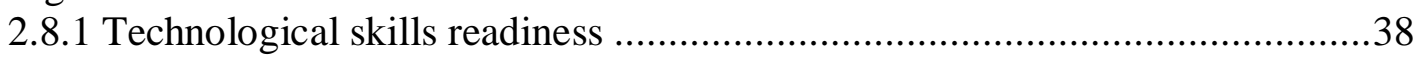

2.8.2 Online learning style readiness ................................................................

2.8.3 Equipment/infrastructure readiness ..............................................................39

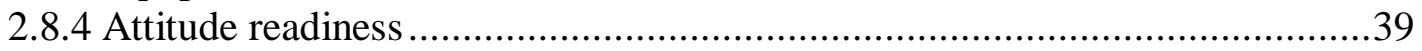

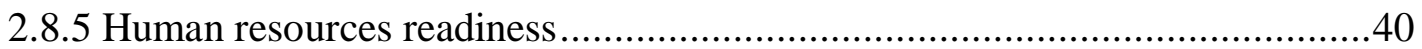

2.8.6 Environmental readiness...................................................................... 41

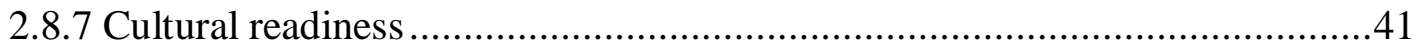

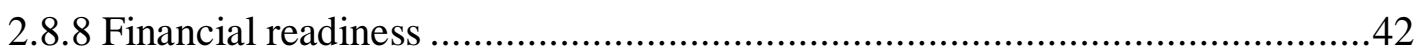

Chapter 3: Research Design and Methods ................................................43

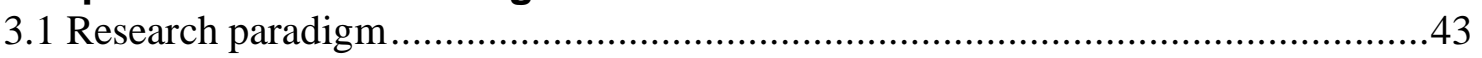




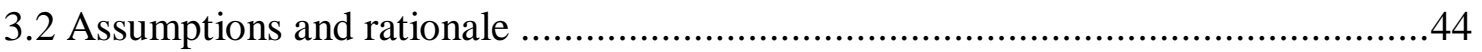

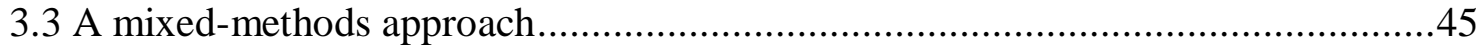

3.3.1 Phase I: Confirmatory - Survey ...................................................................46

3.3.1.1 Quantitative data collection: Population and sample ......................46

3.3.1.2 The instrument .............................................................................48

3.3.1.3 Quantitative data analysis.............................................................48

3.3.1.4 Quantitative validation ...................................................................48

3.3.2 Phase II: Exploratory - Semi-Structured Interviews..................................49

3.3.2.1 Qualitative data collection .............................................................49

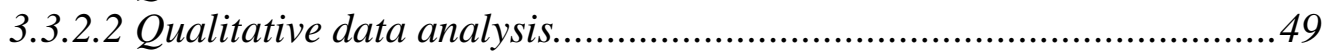

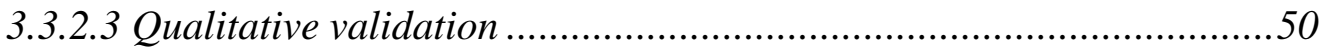

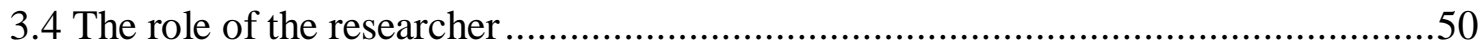

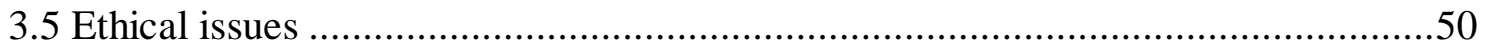

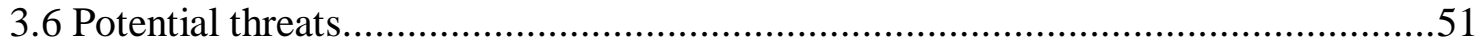

3.7 Written report and presentation using a mixed-methods approach ......................52

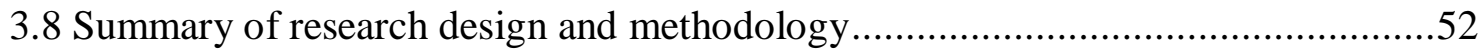

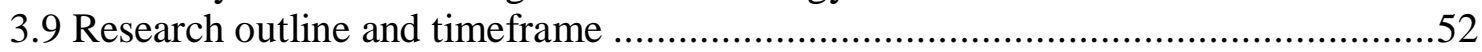

Chapter 4: Phase I - Confirmatory (Survey) ……...................................... 54

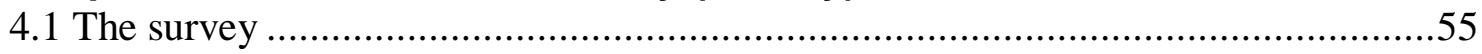

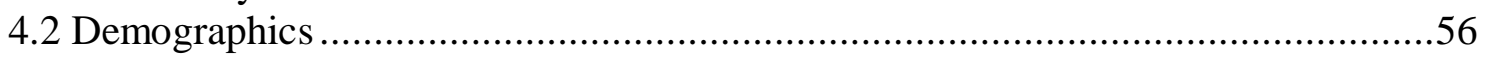

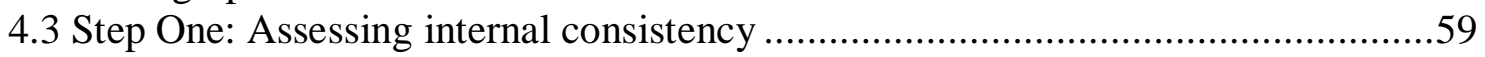

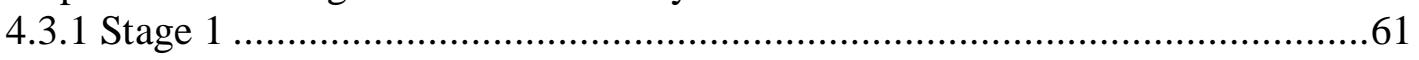

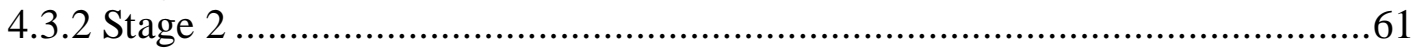

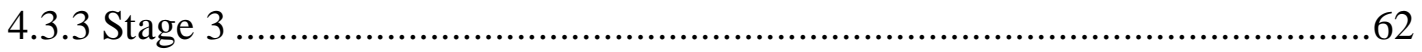

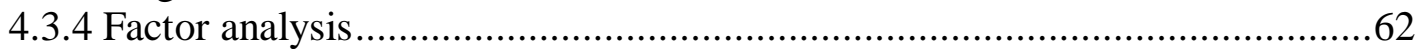

4.3.4.1 Part 1: Assumption checking for factor analysis.............................63

4.3.4.2 Part 2: Factor extraction ..........................................................64

4.3.4.3 Part 3: Factor rotation and interpretation ........................................67

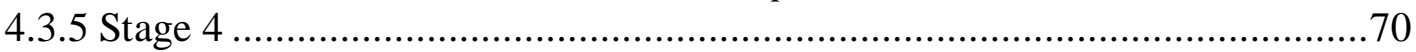

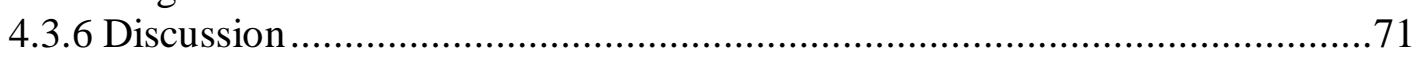

4.4 Step Two: Multiple regression analysis and construct evaluation............................ 72

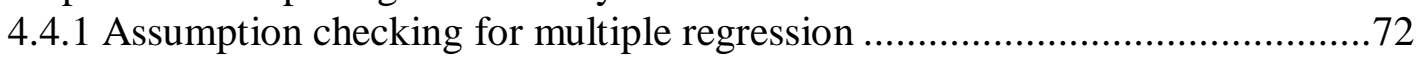

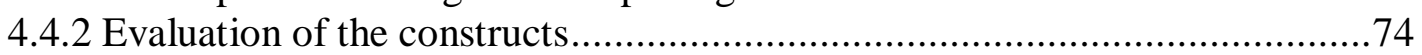

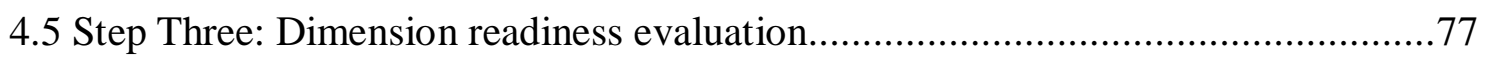

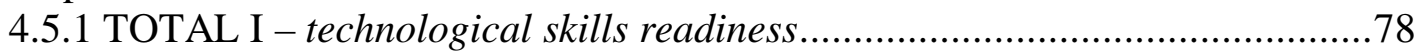

4.5.2 TOTAL IIa - positive online learning style readiness ................................80

4.5.3 TOTAL IIb - negative online learning style readiness................................81

4.5.4 TOTAL III - infrastructurelequipment readiness ......................................8.

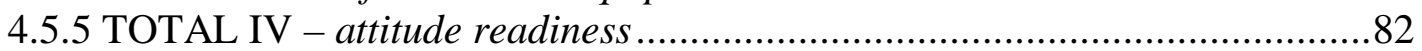

4.5.6 TOTAL V - human resource readiness ....................................................8

4.5.7 TOTAL VI - environmental readiness ....................................................8

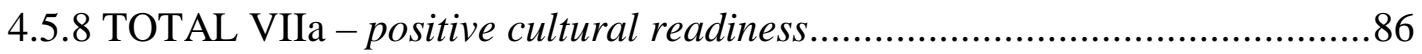

4.5.9 TOTAL VIIb - negative cultural readiness .............................................8

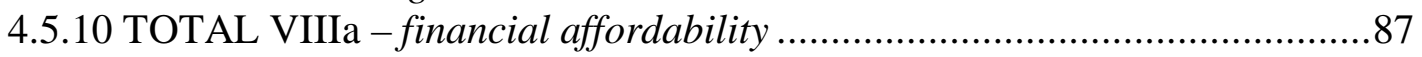

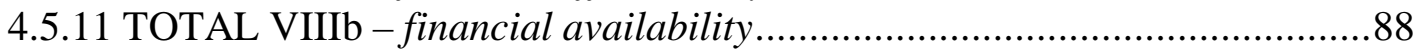

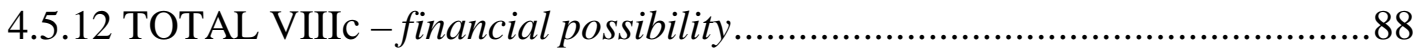

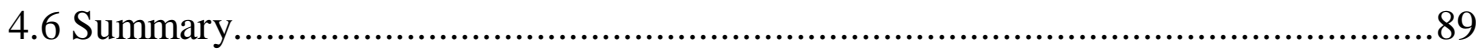

Chapter 5: Phase II - Exploratory (Semi-structured interviews)................93

5.1 Step One: Semi-structured interview development .........................................94 


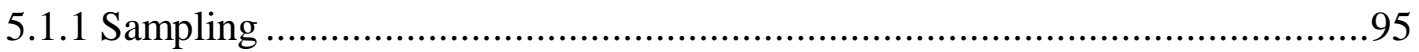

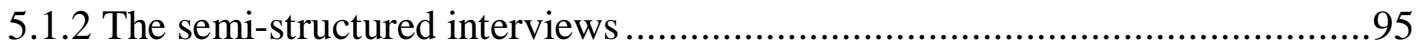

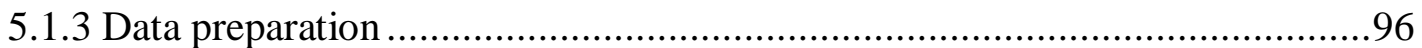

5.2 Step Two: The level of organisational readiness for OES ................................ 97

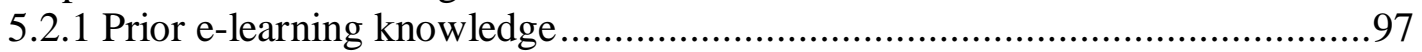

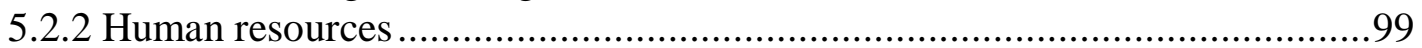

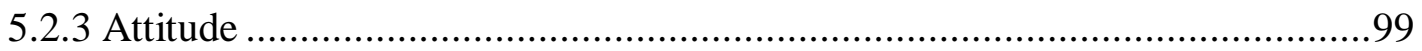

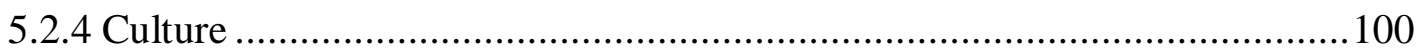

5.2.5 Equipment and infrastructure - Internet connection ................................ 100

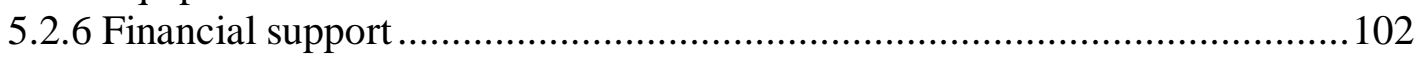

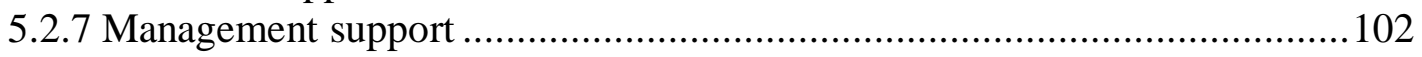

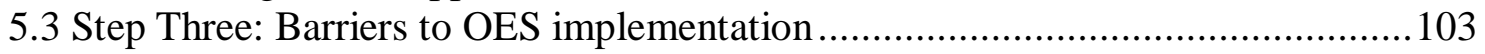

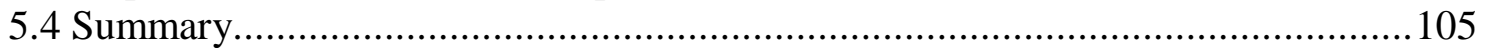

Chapter 6: Conclusions.........................................................................106

6.1 The research findings: Addressing the research questions..................................106

6.1.1 Research question 1: What is the level of organisational readiness for implementing e-learning systems in FORDA?.

6.1.2 Research question 2: What are the critical factors that need to be considered in order to implement successful e-learning systems in FORDA?

6.1.3 Research question 3: What are the key factors that influence organisational readiness for OES in an organisation with no prior elearning knowledge in a developing country context? .............................108

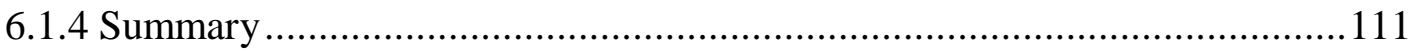

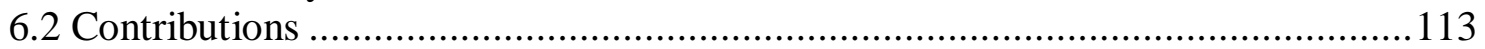

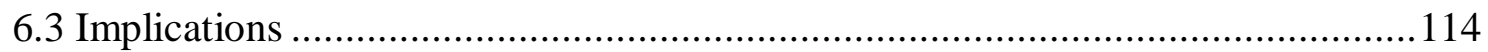

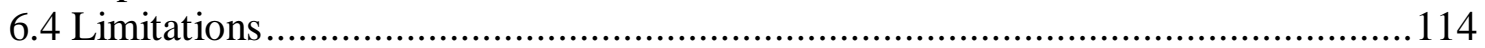

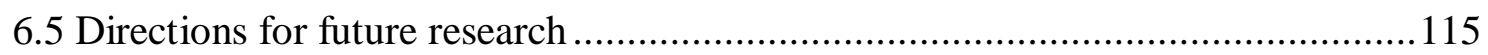

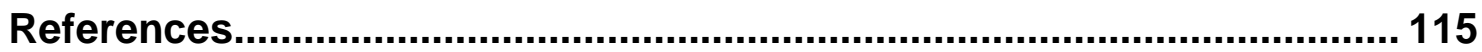

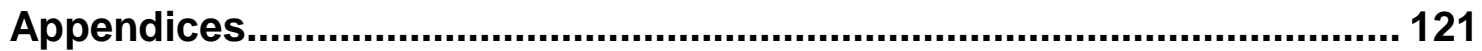

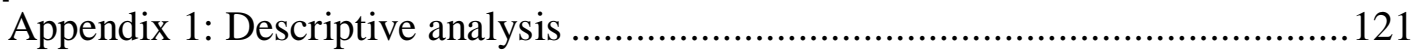

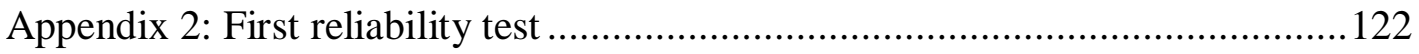

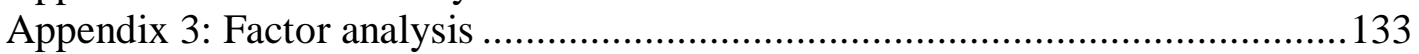

Appendix 4: Fourth reliability test - after factor analysis ................................ 139

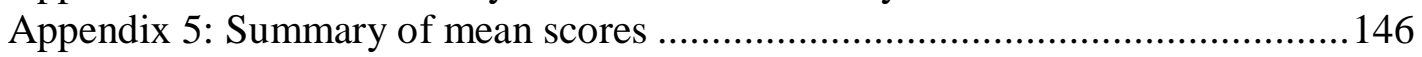

Appendix 6: Assumption checking for multiple regression ..............................148

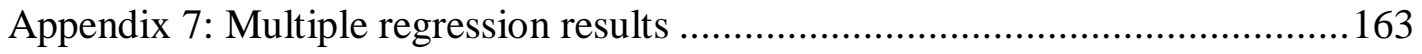

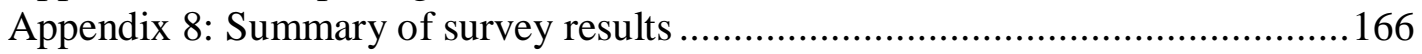

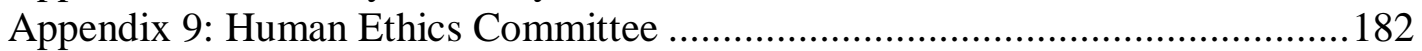

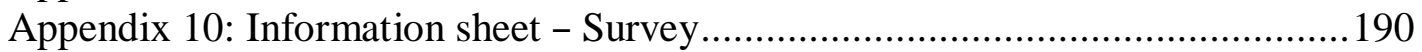

Appendix 11: Information sheet - Semi-structured interview ............................191

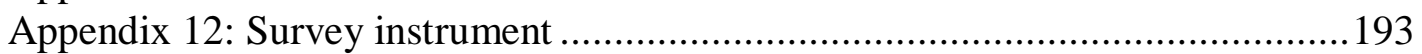

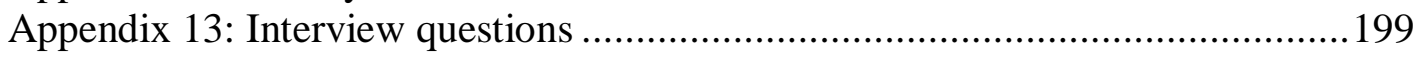




\section{List of Tables}

Table 1. The meanings of E-learning in different contexts......................................13

Table 2. The changing focus of educational technology over the past 30 years ............14

Table 3. The characteristics of Web technologies in effective education.....................15

Table 4. Definitions of various e-learning terms ................................................... 17

Table 5. Summary of e-learning benefits ..........................................................23

Table 6. Critical components in assessing organisational readiness ............................32

Table 7. Summary of e-learning readiness dimensions from nine studies....................33

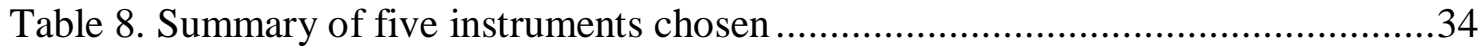

Table 9. Overall means for e-learning readiness among groups of respondents............36

Table 10. Quantitative, qualitative, and mixed methods paradigm assumptions ...........44

Table 11. Sampling frame: Profiles of FORDA .......................................................47

Table 12. Sample size estimation .....................................................................47

Table 13. Minimum returned sample size for a given population size for continuous

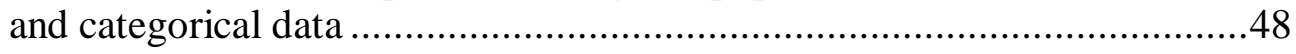

Table 14. Potential threats and how to address them................................................ 51

Table 15. Qualitative and quantitative data collection procedures..............................52

Table 16. Qualitative and quantitative data analysis procedures...............................53

Table 17. Research outline and timeframe (March 2008 - April 2009) .......................53

Table 18. Total number of survey sent/returned and return rate ................................56

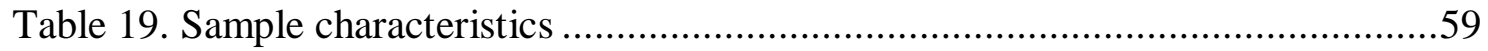

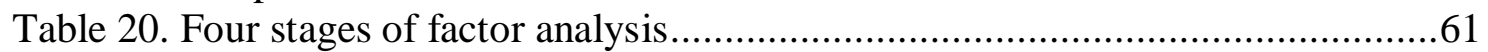

Table 21. Assumption checking for factor analysis..............................................63

Table 22. Scale and subscales after factor analysis ..............................................64

Table 23. Deciding factor extraction ..................................................................65

Table 24. Factor loadings - Dimension II before and after rotation ...........................68

Table 25. Factor loadings - Dimension VII before and after rotation.........................69

Table 26. Factor loadings - Dimension VIII before and after rotation .......................69

Table 27. Component Transformation Matrix .....................................................69

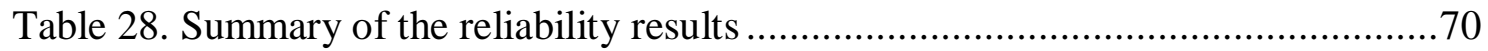

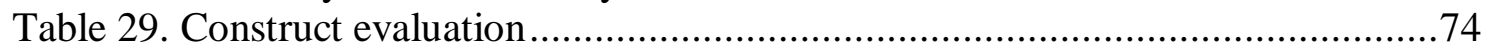

Table 30. Pearson Correlations between twelve scales (dependent variables) ...............76

Table 31. The number of large, medium, and small correlations for each dimension ...76

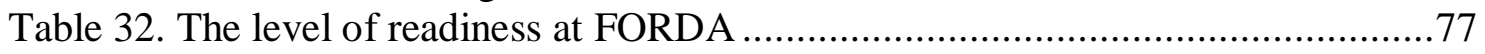

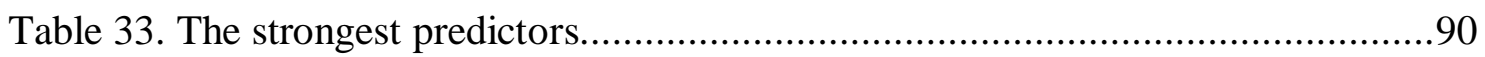

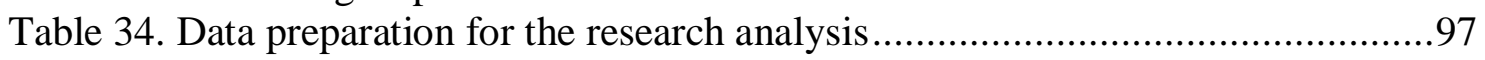

Table 35. Top three barriers for implementing OES in twelve regional offices at

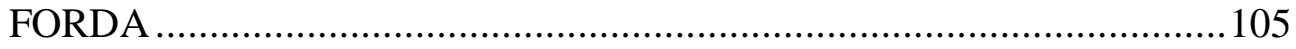




\section{List of Figures}

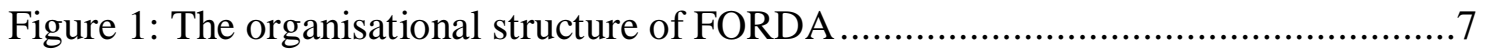

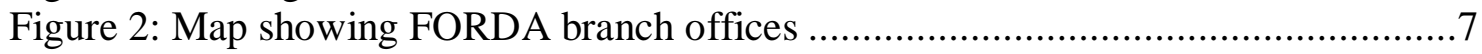

Figure 3: What is e-learning? ............................................................................ 14

Figure 4: Pedagogical stances: Trends and learning paradigm development over

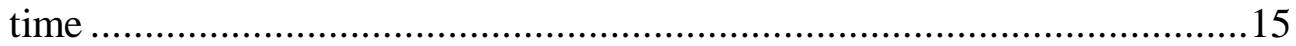

Figure 5: Design framework for the online learning environment ............................ 16

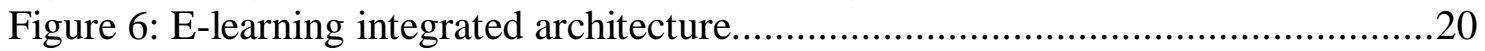

Figure 7: E-Learning Success Model developed (Holsapple \& Lee-Post, 2006, p.

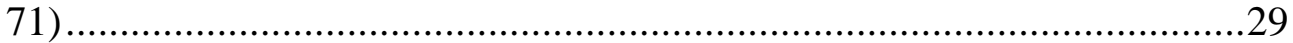

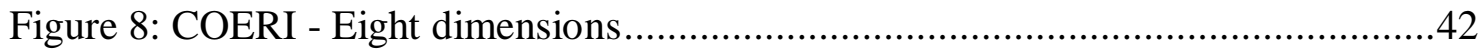

Figure 9: The total number of survey sent and survey returned.................................57

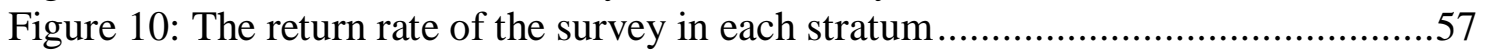

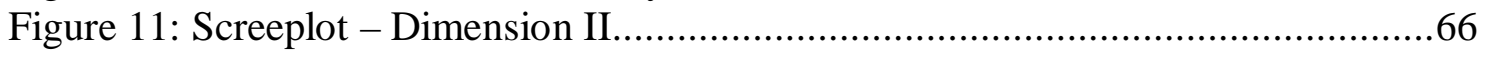

Figure 12: Screeplot - Dimension VII ........................................................66

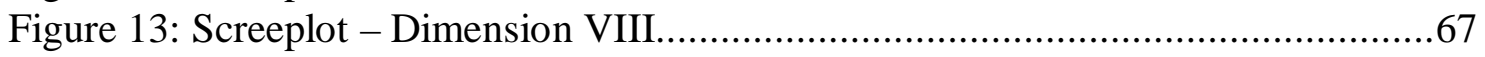

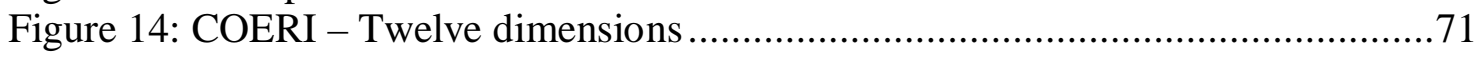

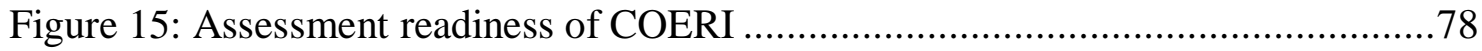

Figure 16: Summary of technological skills readiness ............................................79

Figure 17: Summary of online learning style readiness ...........................................80

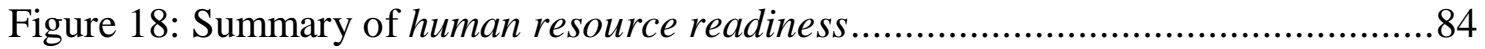

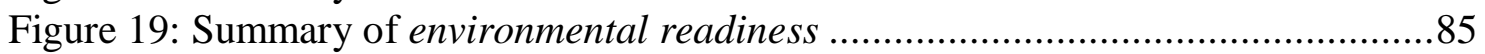

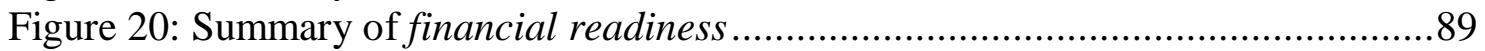

Figure 21: The level of readiness at FORDA......................................................92

Figure 22: Significant predictors that influence e-learning readiness in an organisation with no prior e-learning knowledge - quantitative results.....109

Figure 23: Factors that influence organisational readiness for OES in a developing country context with no prior e-learning knowledge - quantitative results supported by qualitative findings .................................................. 110

Figure 24: Summary of comparison between quantitative results and qualitative findings 


\section{Chapter 1: Introduction}

The purpose of this chapter is to provide a summary of relevant background information for this research. This chapter is organised as follows:

\subsection{Background}

1.2 Statement of the problem

1.3 Research questions

1.4 Purpose of the study

1.5 Research context

1.6 Research design and methodology

1.7 Delimitations and limitations of the study

1.8 The significance of the study

1.9 The structure of the research

\subsection{Background}

E-learning systems are commonly used to boost knowledge and information sharing, $\mathrm{R} \& \mathrm{D}$ collaboration and networks, training and development, and dissemination within organisations. However, before implementing e-learning it is critical to assess the readiness of the organisation for this form of training and development. By identifying factors that need to be considered in developing and implementing e-learning combined with the assessment of organisational readiness, the study attempts to discover successful e-learning implementation strategies particularly at the Forestry Research and Development Agency (FORDA) Indonesia, an R\&D organisation situated in a developing country context with no prior e-learning experience.

This study discusses e-learning in the form of organisational e-learning systems (OES). OES refers to learning activities in an organisation in order to enhance training delivery which is supported by advanced information and communication technology (ICT) without the need for presence in a physical classroom thus across distance and time differences, in which guidance, feedback, assessments, and interaction between learners and instructors are in the form of diverse multimedia through online channels. 
OES has become a significant way to assist knowledge and information sharing throughout organisations. The online components of OES enable people to access information across barriers including physical, time and space in real time and in a timely manner.

At present, training and development has become a significant driver in the spread of knowledge-based organisations. This has forced the growth of OES that allow organisations to continuously engage with a learning environment anywhere and anytime. The role of organisational knowledge and intellectual capital in the competitive strategy of organisations and the desire of organisations to learn more quickly than competitors have encouraged the significant development of e-learning (Bell, Martin, \& Clarke, 2004). One of the key objectives of organisations is creating a culture which enables skilled, enthusiastic and creative people to reach their full potential (Mathieson, 2006). It is widely acknowledged that organisations accomplish training and development in order to be a "learning organisation" that encourages each member of staff to constantly engage in learning with the purpose of continually adapting to the organisation's changing environment (Mathieson, 2006). In 2005, a training and development survey in the Glenmorangie Company in the UK revealed that people potential development has resulted in a $20 \%$ increase in performance; a $50 \%$ reduction in changeover times; and increased operator competency by $40 \%$, leading the company to save $£ 200 \mathrm{k}$ in operations costs (Mathieson, 2006).

In order to successfully implement the systems, it is crucial to assess organisational readiness for e-learning (Chan \& Ngai, 2007; Haney, 2002; Nichols, 2007; Welsh et al, 2003; Wild, Griggs, \& Downing, 2002). Most studies have addressed e-learning readiness measurements in developed countries (Haney, 2002). Research into the topic in the context of developing countries, however, is still lacking (Aydin \& Tasci, 2005). There has been a lack of systematic research on the adoption of e-learning in Asia (Chan \& Ngai, 2007). There have been different rates of adoption and diffusion in the USA, Europe, and Asia (Bell, Martin, \& Clarke, 2004). In this regard, this research attempts to provide an additional insight into the field from the perspective of a developing country. Several studies have developed instruments to assess e-learning readiness for target respondents including: policy makers - managers and CIOs (Abas, Kaur, \& Harun, 2004; Aydin \& Tasci, 2005), providers - e-learning providers (Abas, 
Kaur, \& Harun, 2004), enablers - lecturers, tutors and facilitators (Abas, Kaur, \& Harun, 2004; Sadik, 2007), and receivers - individual learners or trainees (Abas, Kaur, \& Harun, 2004; Pillay, Irving, \& Tones, 2007; Stokes, Cannavina, \& Cannavina, 2004; Watkins, Leigh, \& Triner, 2004). Since most instruments were developed to only assess specific target respondents and/or for developed countries, this study proposes a comprehensive OE readiness instrument (COERI) which can be used to assess all target respondents by combining several existing instruments. COERI is designed to assess OE readiness within a research context with no prior e-learning experience, specifically in a developing country in which OES is still relatively a new topic.

\subsection{Statement of the problem}

FORDA is a large organisation in Indonesia with geographically dispersed offices managing diverse functions and tasks. Each branch office has unique functions and tasks. However, in many cases employees have to work with their counterparts in other offices in order to accomplish their tasks. Efficient and effective ways of communication have become increasingly important to manage the complexity of the organisation.

As a large R\&D organisation, FORDA employs a large number of researchers who work in the forestry field. Some of them work in remote areas, while others may work in more central areas. Some employees may have easy access to online channels, while others may not. Some of the offices have websites and use online means to communicate with their counterparts. However, most of the offices are not fully online. Many offices are still using conventional means of communication such as telephone, fax, and post.

At present, R\&D collaboration, training, and dissemination cannot be provided to the whole organisation. However, collaboration and networking between researchers at FORDA is not fully promoted. Keeping track of who is studying what, which subjects are being investigated, the progress of each study, and the research results is quite problematic. 
Additionally, research collaboration and training are conducted mainly by face-to-face meetings. Researchers mostly use conventional means such as telephone, post, fax, and occasionally email to communicate with their peers. Once the results of a study are published, the institute invites people from other institutions to attend a seminar regarding the research. Some institutions may also ask for assistance with particular subjects and/or particular expertise development. Thus, training may also be held for these specific reasons. This requires people from different places to come together in one place to do the training. The funding for holding these events comes from the organisation's budget. However, it is insufficient for all the researchers to come. Therefore, only one or two people are able to attend the event. This issue is also intensified by time constraints.

The expansion of information and communication technology (ICT), especially the Internet, has significantly fostered the development of online communication among peers. ICT provides opportunities to facilitate the creation, refinement, sharing and use of knowledge effectively between individuals in an online environment. One benefit of using internet-based technology to collaborate online is to decrease the use of paperbased reports. This promotes paperless systems, environmentally friendly systems, and operational efficiencies by reducing the cost of communication in making connections across distributed offices, as well as extending workflows to counterparts throughout the organisation.

However, OES is a complicated system that should be developed carefully and needs a comprehensive approach from all key stakeholders within an organisation that wants to use it. OES involves various aspects: the goals, motivators, resources, and predicted impediments. These aspects will lead to various hurdles for upper level management in making decisions while implementing successful OES in the entire organisation. To address these concerns, it is crucial to assess the organisation's readiness for OES before investing in, developing, and implementing the systems.

This research aims to investigate key issues of organisational readiness faced by FORDA before adopting the new web-based learning techniques. It describes factors that need to be considered before commencing, developing, and implementing OES. Subsequently, a suitable survey instrument is developed to assess FORDA readiness. 
Finally, it identifies these factors as a foundation to prepare suitable learning systems for the FORDA context.

\subsection{Research questions}

The proposed research is designed to assess the readiness of FORDA to implement OES. This will be addressed by three main research questions:

(1) What is the level of organisational readiness for implementing e-learning systems in FORDA?

(2) What are the critical factors that need to be considered in order to implement successful e-learning systems in FORDA?

(3) What are the key factors that influence organisational readiness for OES in an organisation with no prior e-learning knowledge in a developing country context?

\subsection{Purpose of the study}

The research aims to investigate organisational readiness for implementing organisational e-learning systems in FORDA. Subsequently, it intends to determine factors that need to be addressed in order to implement successful OES in the FORDA context. The proposed research will be focused on: reviewing similar instruments from previous studies; developing a comprehensive OE readiness instrument (COERI) for the research context; assessing FORDA's organisational e-learning readiness; and identifying factors that need to be developed further in order to implement successful elearning in FORDA.

\subsection{Research context}

The Forestry Research and Development Agency is part of the Ministry of Forestry in the Republic of Indonesia (MFRI). The main task of MFRI is to administer the natural forest and forestland resources of Indonesia by managing: forest area mapping and configuration; forest exploitation and usability; forest ecological protection (vegetation, land and water conservation); sustainable natural resources and natural environment; and forestry education and training. 
FORDA aims to enhance its function in order to launch its holistic R\&D results to address recent and future issues faced by the forestry sector. One of its strategic plans is to enhance $R \& D$ collaboration and improve the role of knowledge and technology in terms of promoting $\mathrm{R} \& \mathrm{D}$ results in policy making and forestry development.

One of the complementary programmes is to disseminate the results and to promote $R \& D$ collaboration and networks. The main objective is to support forestry R\&D by improving effective dissemination and the benefits of $R \& D$ results as well as developing R\&D networks both nationally and internationally. This includes seeking effective and efficient ways to share, disseminate, and promote the $R \& D$ results. It also incorporates developing and managing productive forestry R\&D networks.

The vision of FORDA is to be the leading institution in providing resources of forestry knowledge and technology in achieving sustainable forest resource management for improving public prosperity. FORDA missions include: improving the capability of forestry knowledge and technology; improving the benefit of information and technology of the R\&D results for decision making and practical forest development; and encouraging organisational, planning and evaluation systems, and the facilities of R\&D.

FORDA has 20 scattered branch offices including one central office (Secretariat of FORDA), four main research and development centres, two research centres, and 13 forestry research institutes. The secretariat office of the FORDA is located in Jakarta, the capital city of Indonesia, whereas the rest of the offices are situated throughout the country.

Building up connections and encouraging collaboration is crucial in a research and development organisation. Therefore, finding effective and efficient ways to build connections, collaboration, and knowledge and information sharing across the organisation is critical. This leads to the significance of building up a powerful means that can connect people and provide knowledge based resources that can be shared throughout the organisation. E-learning allows facilitation of collaboration between individuals despite distance. It enables them to cooperate with their peers and to share 
and transfer their knowledge supported by ICT. The application of e-learning also promotes the social networks between expert individuals throughout the organisation.

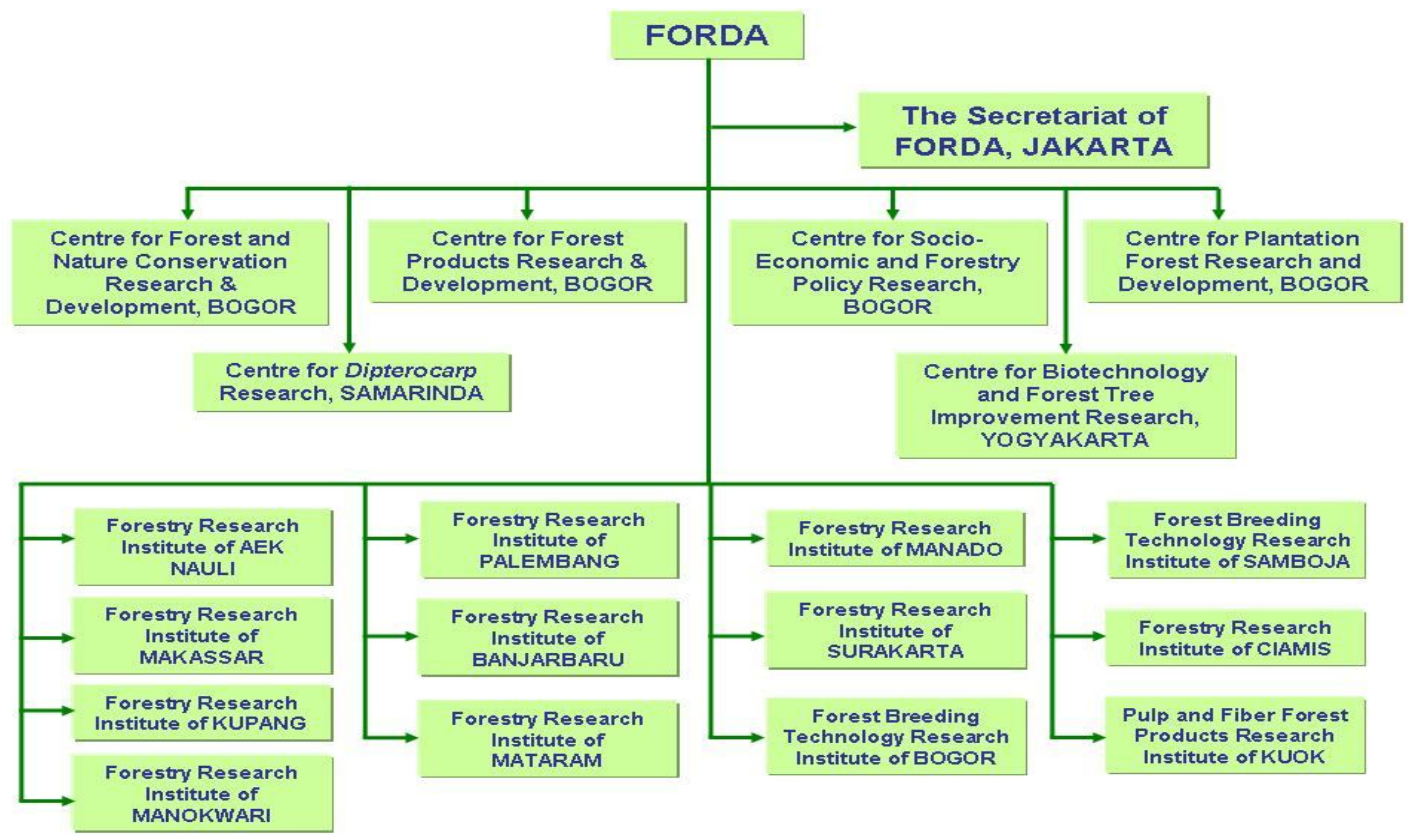

Figure 1: The organisational structure of FORDA

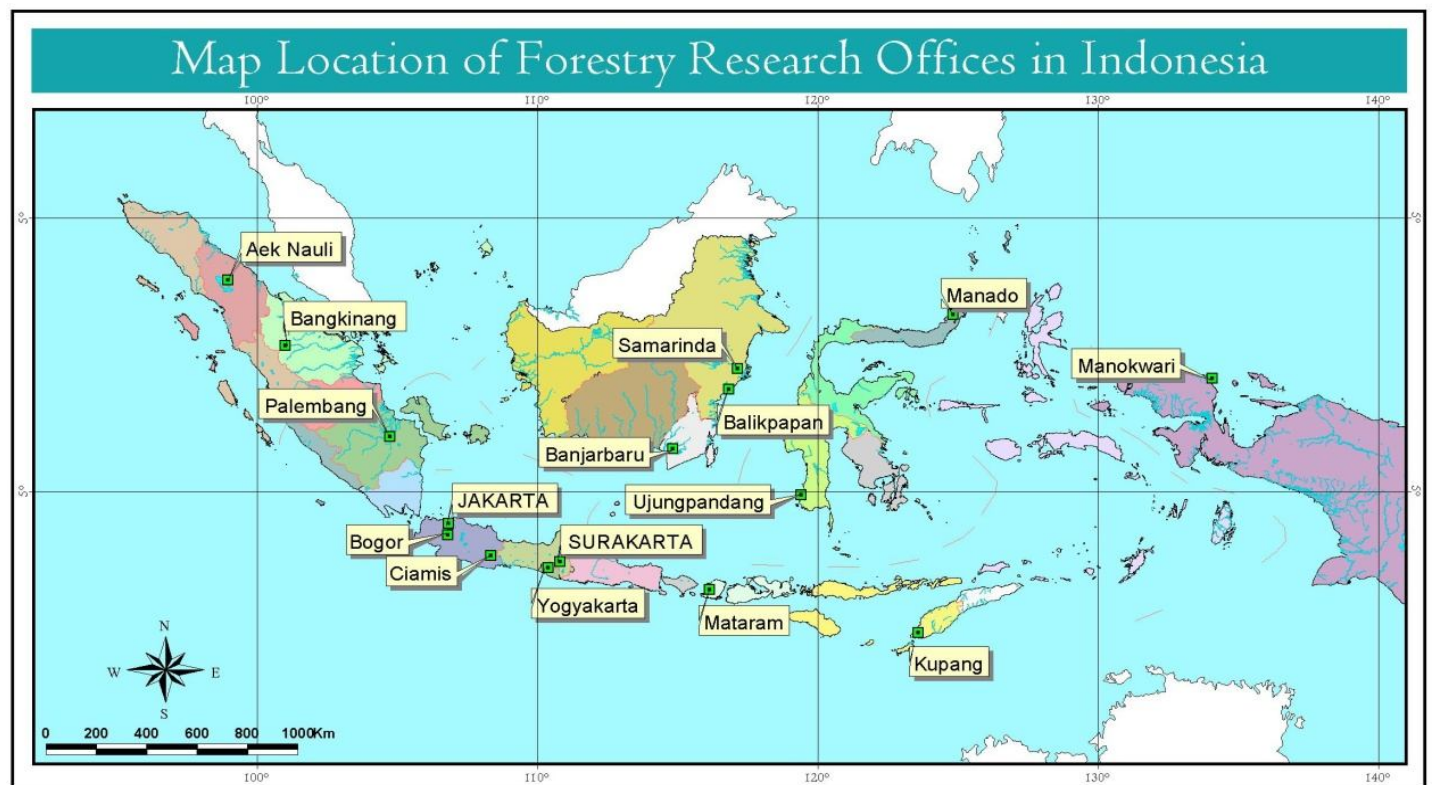

Figure 2: Map showing FORDA branch offices

The researcher conducting this study is an employee of FORDA specifically in the Forestry Research Institute (FRI) of Surakarta located in Central Java, Indonesia. The previous role of the researcher was to organise the communication and dissemination of research results. 


\subsection{Research design and methodology}

The research employed a mixed-method approach. It was a cross-sectional study as it took place at a single point in time. It was based on the Positivist tradition as most of the data were collected through a cross-sectional survey, followed by 12 semi-structured interviews to complement the survey results. Both quantitative and qualitative data were collected, analysed, and interpreted. The quantitative data were collected from all levels of FORDA employees, whereas the qualitative data were collected from policy makers at FORDA. The results of both methods were integrated in the final phase as shown in the discussion and interpretation chapter. The survey was delivered from early August 2008 until late November 2008. The semi-structured interviews were conducted in August 2008. A cover sheet that provides a brief research description, instructions, and an introduction to the researcher were added to the survey instrument and the interview consent sheet. The survey instrument was delivered both online and on paper.

\subsection{Delimitations and limitations of the study}

The study was restricted to the FORDA context in Indonesia. Primarily, it surveyed a number of workers from 20 scattered FORDA branch offices. Secondly, to complement the survey results and to gain deeper understanding of the issue in a natural setting, 12 FORDA policy makers were interviewed. The main limitations of the study were in time and resources. The research samples were taken from 20 different locations across a range of areas. Due to limited funding and time, the survey was delivered by online means and by post. Several issues may arise in relation to the survey delivery. Although it saves time and money, not all participants will be familiar with online surveys. Moreover, the online infrastructure may not yet be available. On the other hand, compared to the online version, paper-based surveys are costly and time consuming. However, they can be used to reach people at remote locations with no online infrastructure and people who are not familiar with online tools. English is not the first language in Indonesia. Therefore the translation (of the information sheet, survey instrument, and interview questions) took considerable time and effort. To save time, the qualitative analysis was done in Indonesian, and then the results were translated into English. It was also quite challenging to find the most suitable respondents in the 
organisation. Due to the nature of developing countries, the research relied heavily on conventional means of communication such as post and telephone.

\subsection{The significance of the study}

There are four main goals of this study. Firstly, by determining the organisation's readiness for OES, it will provide guidance for policy makers to develop OES that are best suited to the level of readiness of the organisation. Secondly, the study will provide deeper understanding of a new OE-based system that can be implemented particularly in the FORDA context to improve organisational practices including providing training, sharing organisational knowledge and information, and promoting dissemination of research results. Thirdly, the study will also be useful as the initial internal assessment is conducted by the researcher who is also an employee of the FORDA. This will provide a better foundation before continuing with external assessment (for example by e-learning vendors or consultants) in order to develop successful OES in the future. Finally, the study will enrich the existing body of knowledge specifically in the information systems field which will be valuable for researchers and practitioners for measuring OE readiness in institutions particularly in the developing countries.

\subsection{Definitions of key terms}

The definitions presented below are based on the literature review that informed the study.

E-learning: Learning activities supported by the use of advanced information and communication technology (ICT) in which during the learning process either learners or instructors are not required to be present in a physical classroom, so they can be conducted across distance and time differences, and in which the assessments, guidance, and feedback can be delivered in the form of diverse multimedia through online channels.

Organisational e-learning systems (OES): Learning activities in an organisation supported by the use of advanced ICT for enhancing organisational training, in which during the learning process either learners or instructors are not required to be present in 
a physical classroom, so they can be conducted across distance and time differences, and in which the assessments, guidance, and feedback can be delivered in the form of diverse multimedia through online channels.

OES readiness: The level of mental and physical preparedness of an organisation to adopt and implement e-learning systems in order to enhance training within the organisation.

OES readiness evaluation: The measurement of the level of mental and physical preparedness of an organisation to adopt and implement e-learning systems in order to enhance training within the organisation.

\subsection{The structure of the research}

The research is organised into seven chapters as follows:

Chapter 1 provides the introduction including the statement of the problem, the purpose of the study, the research context, key definitions, research questions, a brief description of the research design and methods, limitations and delimitations, and the significance of the proposed research.

Chapter 2 presents the literature review to address the nature of e-learning systems and organisational e-learning readiness measurement.

Chapter 3 addresses the research design and methodology for the study.

Chapter 4 presents quantitative results, discussion, and interpretations.

Chapter 5 presents the qualitative results, discussion, and interpretations.

Chapter 6 provides discussion and interpretation of both quantitative results and qualitative findings as well as the conclusions of the study including contributions, initiatives, and future directions for related research. 


\section{Chapter 2: Literature Review}

\subsection{Introduction}

This section presents the nature of organisational e-learning systems (OES) and organisational readiness for OES. It also discusses existing instruments to assess elearning readiness from several studies. The gaps found are also presented in this chapter. This chapter is organised as follows:

2.1 Introduction

2.2 Justification for examining organisational readiness for OES

2.3 The nature of e-learning

2.4 Critical success factors for developing and implementing OES

2.5 Assessing organisational readiness for OES

2.6 E-learning readiness instruments

2.7 The gaps

2.8 Eight dimensions of OES readiness

\subsection{Justification for examining organisational readiness for OES}

The continuing growth in use of ICT, particularly the Internet, has promoted the ability to adopt global e-learning practices. The Internet is an effective tool providing accessible information to diverse users from different places. It is a vital means for the survival and growth of organisations in the competitive global market. It enables organisations to build their image and promote their image internationally. Chan and Ngai (2007) noted that the Internet has revealed a new dimension of distance learning by providing a new mechanism to deliver training involving strategic tools to enhance training delivery and to improve organisations' performance in optimising efficiencies.

The significant expansion of e-learning has been intensified by considerable cost reduction of the technologies, increased processing power, extended network and communications infrastructure, and the utilisation of the Internet and World Wide Web (WWW) (Chan \& Ngai, 2007; Sharma \& Mishra, 2007; Welsh et al., 2003; White, 2007). ICT literacy is increasingly important for future employment. Condie and 
Livingston (2007) stated that people are not only required to have knowledge and skills in handling new technologies but also to learn through computers and the available networks via the Internet. Moreover, Bell, Martin, and Clarke (2004) noted that the vital role of organisational and intellectual capital also affects OES adoption in corporate organisations worldwide.

Currently, the e-learning industry is the fastest growing sub-sector of the approximately $\$ 2.3$ trillion global education market; while the online higher education market is projected to go beyond $\$ 69$ billion by 2015 (Sharma \& Mishra, 2007). The increased demand for e-learning in higher education and training is associated with the change from a labour intensive workforce to a more globalised knowledge intensive workforce (Chan \& Ngai, 2007; Sharma \& Mishra, 2007). This has resulted in rapid growth and expansion of knowledge-based industries in business and academic fields with a transforming influence on education and corporate training and development worldwide over the past decade (Chan \& Ngai, 2007).

To successfully implement OES it is crucial to assess organisational readiness for OES, particularly in organisations with no prior e-learning knowledge. Haney (2002) noted that before initiating, implementing, and using OES, it is important to assess organisational readiness for the systems by recognising the organisation's goals, needs, motivators, resources and constraints. Potential hurdles may appear during OES initiation and implementation. The assessment should include all stakeholders at all levels (from low level employees to policy makers) within an organisation that is considering implementing OES. Before assessing organisational readiness for OES, it is important to understand the nature of OES and the critical success factors for initiating, developing and implementing OES.

\subsection{The nature of e-learning}

\subsubsection{What is e-learning?}

Previous studies provide diverse definitions of and synonyms for e-learning. People may have their own interpretation of each term they choose to use. Nicholson (2007) revealed that since the 1960 s, there have been several evolutionary paths and definitions of e-learning. His study confirms that e-learning has evolved in many different ways in 
different sectors including business, education, training, and the military, and that each sector has different meanings for e-learning. He also found that for the past 40 years, educators and trainers at all levels across all sectors had utilised computers in different ways to support and enhance teaching and learning, thus e-learning has different meanings in different contexts. E-learning in the business, higher education, and training sectors involves Internet-based flexible delivery of content and programmes that focus on sustaining particular communities of practice. Table 1 shows the meanings of e-learning in different contexts.

Table 1. The meanings of E-learning in different contexts

\begin{tabular}{|l|l|}
\hline \multicolumn{1}{|c|}{ Sectors } & \multicolumn{1}{c|}{ Refers to } \\
\hline School & The use of both software-based and online learning \\
\hline Higher education & A range of online practices (Development of meta-cognitive skills; focus on reflective collaboration learning) \\
\hline Business & $\begin{array}{l}\text { A range of online practices (improved productivity; cost reduction specifically for global business environment; } \\
\text { focus on collaborative productivity) }\end{array}$ \\
\hline Training & $\begin{array}{l}\text { A range of online practices (improved productivity and cost reduction specifically for global business } \\
\text { environment) }\end{array}$ \\
\hline Military & A range of online practices \\
\hline
\end{tabular}

(Summarised from Nicholson, 2007)

Various terms have been used including: computer assisted instruction, computer-based training, computer managed instruction, course management system, integrated learning systems, interactive multimedia instruction, learning management system, technology based learning, technology enhanced learning, web-based training, and so forth.

Several studies have attempted to provide a comprehensive definition of e-learning from different perspectives. Researchers have used different methods to define the term including investigating the history of e-learning; comparing the meanings from different contexts and practices; investigating the technologies employed in the systems; developing frameworks; and examining e-learning theoretical positions (pedagogy) in each era.

The technologies and learning paradigms have evolved over a period of time. Nicholson (2007) noted that to understand each technology and to match each technology with appropriate approaches for teaching and learning, e-learning can be differentiated into four eras as shown in Table 2. Sharma and Mishra (2007) revealed that e-learning pedagogy is the science and art of teaching and learning by utilising the Internet and the Web. 


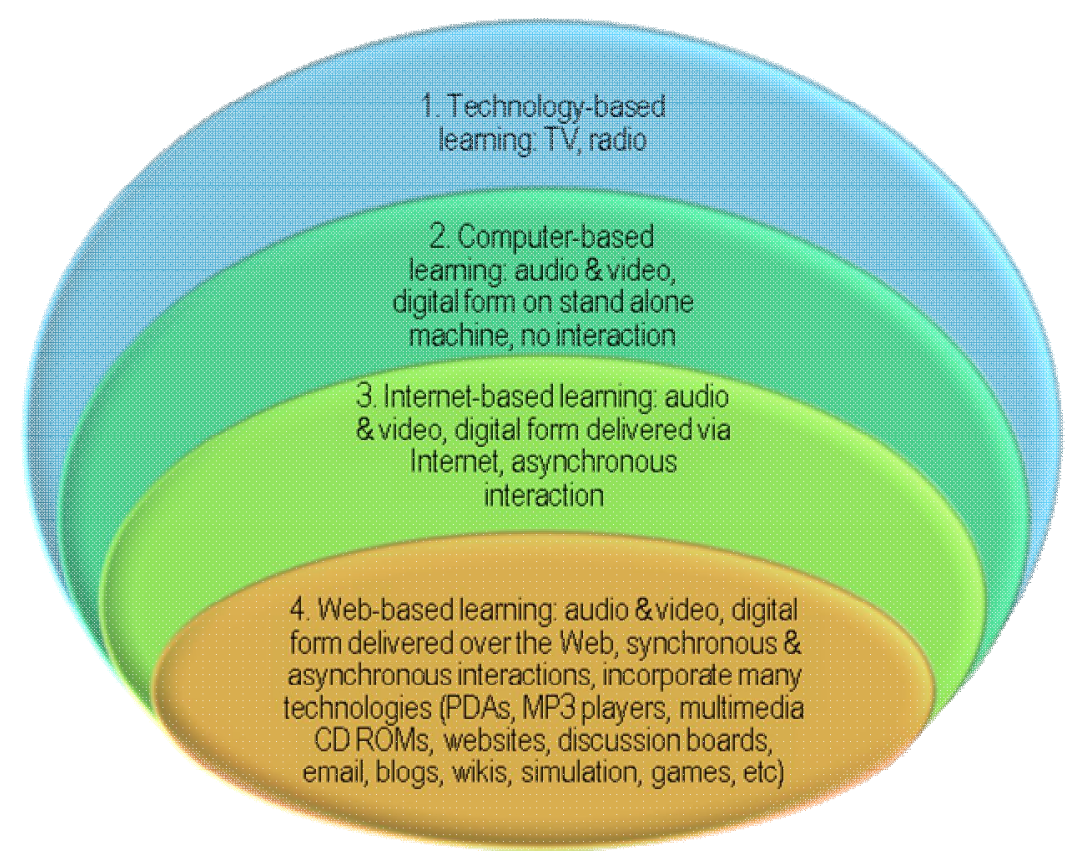

Figure 3: What is e-learning?

(Modified from Sharma \& Mishra, 2007, p. 4)

Table 2. The changing focus of educational technology over the past 30 years

\begin{tabular}{|c|l|l|l|}
\hline \multicolumn{1}{|c|}{ Era } & \multicolumn{1}{|c|}{ Focus } & \multicolumn{1}{c|}{ Approaches } & \multicolumn{1}{c|}{ Educational characteristics } \\
\hline $1975-1985$ & $\begin{array}{l}\text { Programming; drill and } \\
\text { practice; computer- } \\
\text { assisted learning (CAL) }\end{array}$ & Behaviourism & $\begin{array}{l}\text { Behaviourist approaches to learning and instruction; } \\
\text { Programming to build tools and solve problems; } \\
\text { Local user-computer interaction }\end{array}$ \\
\hline $1983-1990$ & $\begin{array}{l}\text { Computer-Based Training; } \\
\text { Multimedia }\end{array}$ & $\begin{array}{l}\text { Constructivism - } \\
\text { passive learner } \\
\text { models } \\
\text { Passive learner models dominant; } \\
\text { Constructivist influences begin to appear in educational software } \\
\text { design and use }\end{array}$ \\
\hline $1990-1995$ & Web-Based Training & $\begin{array}{l}\text { Constructivism - } \\
\text { Active learner } \\
\text { models }\end{array}$ & $\begin{array}{l}\text { Internet-based content delivery; } \\
\text { Active learner models developed; } \\
\text { Constructivist perspective common; } \\
\text { Limited end-user interactions }\end{array}$ \\
\hline $1995-2005$ & E-Learning & $\begin{array}{l}\text { Constructivism and } \\
\text { cognitivism }\end{array}$ & $\begin{array}{l}\text { Internet-based flexible courseware delivery; } \\
\text { Increased interactivity; } \\
\text { Online multimedia courseware; distributed constructivist and } \\
\text { cognitivist models common; } \\
\text { Remote user-user interactions }\end{array}$ \\
& & \multicolumn{2}{|l}{} \\
\hline
\end{tabular}

Adapted and Modified from Nicholson (2007, p. 7)

According to Nicholson (2007) pedagogical stances have changed over a period of time (Figure 4) from didactic, to interactive local, to collaborative distributed; likewise the learning paradigms have also evolved from cognitive, to constructivist, to social constructivist. Constructivism, particularly social constructivism is the most obvious adopted paradigm in all areas including educational, business, and training applications (Nicholson, 2007; Sharma \& Mishra, 2007). 


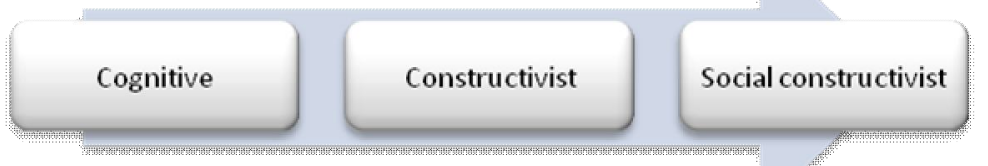

Time

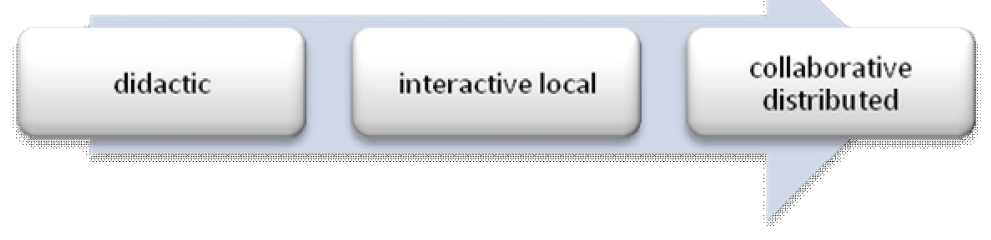

Figure 4: Pedagogical stances: Trends and learning paradigm development over time

Nicholson (2007) categorised the e-learning theoretical position as active learnercentred pedagogies focusing on online contexts and including the full array of computer-based learning platforms and delivery methods, genres, formats, and media (multimedia, educational programming, simulations, games, and new media - either fixed or mobile platforms) across all disciplines. Stokes et al. (2004) also noted that elearning is typically a student-centred approach related to constructivism positing knowledge is constructed by learners through social interaction with others. To date, Web-constructivism is identified as a new e-learning paradigm involving the effective utilisation of the Internet and the WWW in education that provides participants with network technology enabling them to communicate, cooperate, collaborate, and interact with each other (Nicholson, 2007; Sharma \& Mishra, 2007; Stokes et al., 2004). Table 3 shows the unique characteristics of the Web features in effective education.

Table 3. The characteristics of Web technologies in effective education

\begin{tabular}{|l|l|}
\hline Web Characteristics & Description of characteristics \\
\hline Physicality of the media & No longer paper-based \\
\hline Social interactions & Lack of physical co-presence both increases and limits interaction \\
\hline Conversational pragmatics & Being online, time independent, and flexible, increases participation and dialogue \\
\hline Diversity of resources & Available on the net \\
\hline Lack of permanence & Of materials \\
\hline Questionable authenticity & Of materials \\
\hline Multimodality & Of learning resources, available in different forms: text, graphics, audio, video, animations \\
\hline Hypermedia - based & Not linear \\
\hline Customisation and personalisation & Participants can learn from anywhere, anytime, and choose the methods that best suit them \\
\hline
\end{tabular}

Summarised from Sharma and Mishra (2007) 


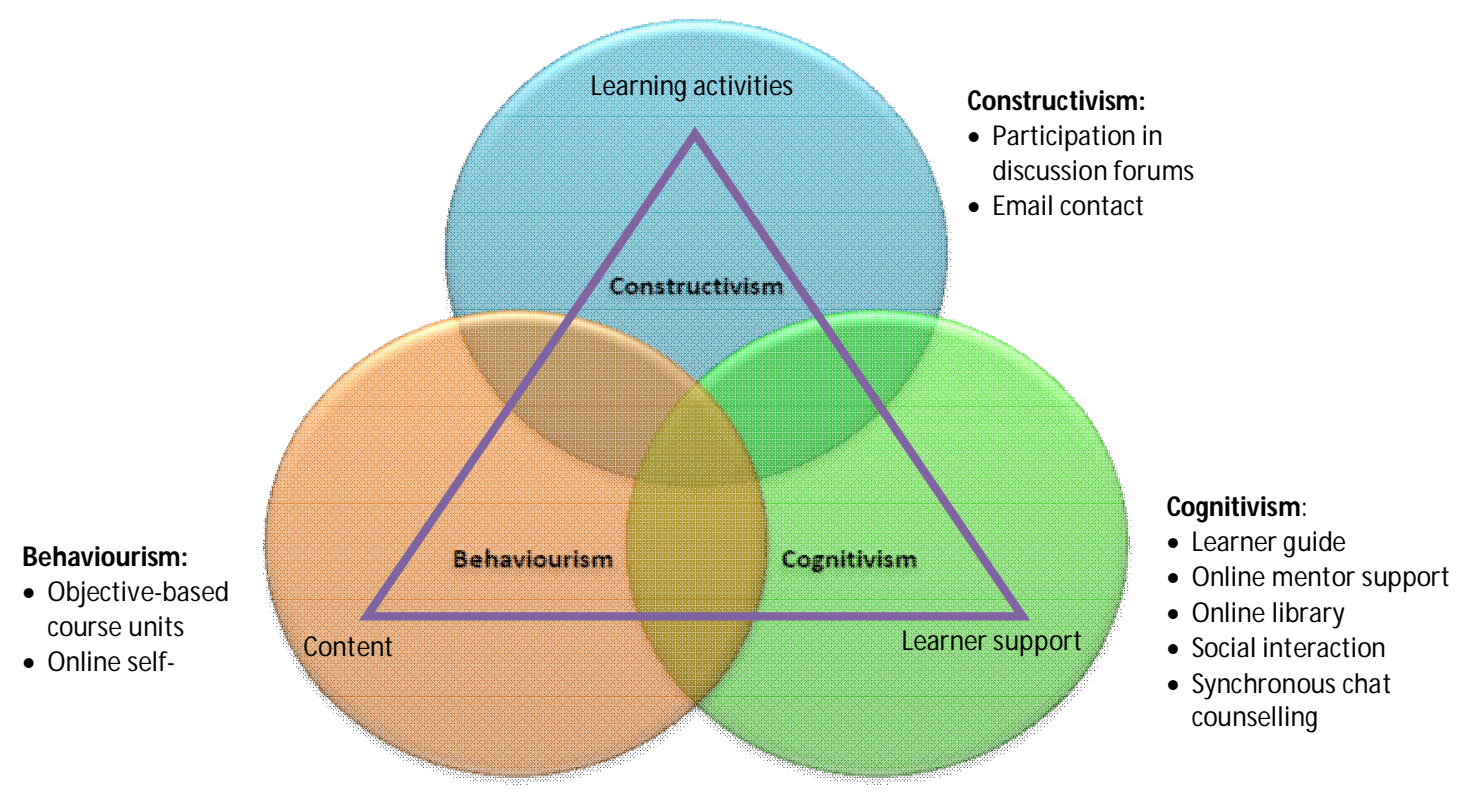

Figure 5: Design framework for the online learning environment (Taken from Sharma \& Mishra, 2007, p. 7)

Sharma and Mishra (2007) noted that the new e-learning environment involves the flexible delivery option and provides learning experiences to be accessed by the learner anywhere anytime including reading materials on the Web, listening to audio, watching video, and experiencing animations and simulations, followed by learner reflection. The learner reflection activity should be supported by both synchronous and asynchronous media to enable interaction and facilitation of knowledge construction through group work, projects, presentations, and other creative activities.

Table 4 presents various definitions of e-learning from previous studies. Having examined all these definitions, the term organisational e-learning systems (OES) in this study refers to learning activities in an organisation supported by the use of advanced information and communication technology (ICT) in which during the learning process either learners or instructors are not required to be present at a physical classroom across distance and time differences, and in which the assessments, guidance, and feedback can be delivered in the form of diverse multimedia through online channels. 
Table 4. Definitions of various e-learning terms

\begin{tabular}{|c|c|}
\hline Terms and Definitions & References \\
\hline \multicolumn{2}{|l|}{ E-learning (Electronic Learning) } \\
\hline $\begin{array}{l}\text { "the use of digital technologies and media to deliver, support, and enhance teaching, learning, } \\
\text { assessment and evaluation" (Armitage \& O'Leary, 2003, p. 4) } \\
\text { "the systematic use of networked information and communication technology in teaching and } \\
\text { learning" (Naidu, 2003, p. 5) }\end{array}$ & Sharma \& Mishra (2007) \\
\hline $\begin{array}{l}\text { A new form of learning that uses the ability of the Internet to deliver customised, often interactive, } \\
\text { learning materials and programmes to diverse local and distant communities of practice }\end{array}$ & Nicholson (2007) \\
\hline $\begin{array}{l}\text { "an instructional content or learning experiences delivered or enabled by electronic technology } \\
\text { particularly computer networks, and standalone computers (The Commission on Technology and } \\
\text { Adult Learning, 2001)" (p. 244) }\end{array}$ & Aydin \& Tasci (2005) \\
\hline $\begin{array}{l}\text { "the process of extending learning or delivering instructional materials to remote sites via the Internet, } \\
\text { intranet/extranet, audio, video, satellite broadcast, interactive TV, and CD-ROM" (p. 68) }\end{array}$ & Holsapple \& Lee-Post (2006) \\
\hline "The acquisition of knowledge and skills through mediated information and instruction" & $\begin{array}{l}\text { United States Distance } \\
\text { Learning Association, } 2001\end{array}$ \\
\hline $\begin{array}{l}\text { "teaching and learning delivered, enabled or mediated by electronic technology for the explicit } \\
\text { purpose of learning" (Rossen \& Hartley, 2001, p. 2). They include online learning, Web-based } \\
\text { learning, and computer-based learning within e-learning }\end{array}$ & $\begin{array}{l}\text { The American Society for } \\
\text { Training and Development } \\
\text { (ASTD) (2001) }\end{array}$ \\
\hline $\begin{array}{l}\text { "the use of computer network technology, primarily over an intranet or through the Internet, to deliver } \\
\text { information and instruction to individuals" p. } 246 \\
\text { "any system that generates and disseminates information and is designed to improve performance" p. } \\
246\end{array}$ & Welsh, et al., 2003 \\
\hline $\begin{array}{l}\text { "A range of activities, from the effective use of digital resources and learning technologies in the } \\
\text { classroom, through to a personal learning experience enabled through individual access at home } \\
\text { or elsewhere" p. } 340\end{array}$ & Condie \& Livingston, 2007 \\
\hline \multicolumn{2}{|l|}{ Rapid e-learning } \\
\hline $\begin{array}{l}\text { "the rapid creation of e-learning content by SMEs (subject matter experts) - those who use easy to } \\
\text { understand software tools to create content and are able to publish finished pieces in numbers of } \\
\text { weeks" }\end{array}$ & Unneberg, 2007 \\
\hline \multicolumn{2}{|l|}{ WBT (Web Based Training) } \\
\hline $\begin{array}{l}\text { "a training method for distance learning that uses the technology of the Web, the Internet, intranets, } \\
\text { and extranets" } \\
\text { "the communication of information via the World Wide Web (WWW) with the intention of providing } \\
\text { instruction" }\end{array}$ & Chan \& Ngai (2007) \\
\hline \multicolumn{2}{|l|}{ Web-Based Instruction } \\
\hline $\begin{array}{l}\text { "a hypermedia based instructional programme, which utilises the attributes and resources of the } \\
\text { World Wide Web to create a meaningful learning environment where learning is fostered and } \\
\text { supported" (Khan, 1997, p. 6) }\end{array}$ & Sharma \& Mishra (2007) \\
\hline \multicolumn{2}{|l|}{ Virtual Learning } \\
\hline $\begin{array}{l}\text { "the educational process of learning over the Internet without having face-to-face contact" However } \\
\text { some virtual learning may also include tele-learning (French, et al., 1999, p. 2) }\end{array}$ & Sharma \& Mishra (2007) \\
\hline \multicolumn{2}{|l|}{ Online Learning } \\
\hline $\begin{array}{l}\text { "synonymous to web-based learning where learning is fostered via WWW only, in an intranet or } \\
\text { Internet. It has been recognised as the new generation in the evolutionary growth of open, } \\
\text { flexible, and distance learning" (Mishra, 2001) }\end{array}$ & Sharma \& Mishra (2007) \\
\hline \multicolumn{2}{|l|}{ Technology-based training } \\
\hline $\begin{array}{l}\text { "Corporate e-learning report defines e-learning as 'a wide set of applications and processes including } \\
\text { computer-based learning, virtual classroom, and digital collaboration' (Hambrecht \& Co., 2000, } \\
\text { p.8) }\end{array}$ & Sharma \& Mishra (2007) \\
\hline \multicolumn{2}{|l|}{ Distance Learning } \\
\hline $\begin{array}{l}\text { "Current telecommunications and information technologies provide the indispensable capabilities for } \\
\text { lifelong education without the need for presence at a physical classroom" }\end{array}$ & $\begin{array}{l}\text { Maglogiannis \& Karpouzis, } \\
2007 \text {, p. } 23\end{array}$ \\
\hline
\end{tabular}

\subsubsection{What are the characteristics of e-learning?}

Several types of distance education courses have been identified: (1) Correspondence conducted via the postal service; (2) Internet conducted either synchronously or asynchronously; (3) Tele-course/Broadcast where content is delivered via radio or television; (4) CD-ROM where the student interacts with computer content stored on a CD-ROM; and (5) Pocket PC/Mobile Learning where the student accesses course content stored on a mobile device or through a wireless server. 
Haney (2002) noted that e-learning is a multifaceted system not only involving webbased courses but also incorporating skill and competency development based in wide set of technology-based applications and processes. The processes include web-based learning, computer-based training, virtual classrooms, and digital collaboration, while the course content is delivered through the Internet, intranet/extranet, local servers, individual computers, and CD-ROMs (Haney, 2002).

The notion of e-learning often relates to open education (Maglogiannis \& Karpouzis, 2007) or an open course programme (Mathieson, 2006) that offers courses matched to individuals' requirements. The programme allows both instructors and learners to not be in the same space nor at the same time, so that the process of teaching and learning is performed and supported by synchronous (chat rooms and scheduled events) and asynchronous (discussion boards, email newsletters, newswires, polls and surveys, and directories) means of communication.

Nichols (2007) and Sharma and Mishra (2007) identified types of e-learning utilisation in educational institutions: (1) On-site campus - integrating classroom teaching that works as a supplement to face-to-face teaching; (2) Mixed-mode (blended learning) complementing face-to-face teaching with distance education methods in a hybrid form of flexible blended delivery; and (3) Distance education - an independent teaching and learning mode that replaces face-to-face teaching. In this regard, e-learning can be categorised into synchronous learning, blended learning, and asynchronous learning.

Maglogiannis and Karpouzis (2007) defined synchronous learning as a learning environment in which both learners and instructors are present at the same time during the instruction in different places. Welsh et al. (2003) noted that the systems require both parties to be in front of their computers at the same time. Synchronous refers to simultaneous two way communication with practically no time delay, allowing participants to interact with each other and respond in real time. Synchronous means includes chat rooms, scheduled event discussions and the use of instant messaging applications (Skype, Yahoo Messenger, MSN) enabling participants to type comments and replies in real time. It can be effectively applied by running scheduled online events involving instructors or guest speakers followed by publishing an edited transcript as a 
follow up. Therefore, it allows other participants to catch up by reading the transcripts if they were not present in the chat room.

Blended learning is a combination of online and face-to-face content delivery using various learning resources and communication options available to learners and instructors (Davis \& Fill, 2007; Kaczynski, Wood, \& Harding, 2008). It involves a mix of delivery options. It combines both asynchronous and synchronous means and uses a combination of technology and classroom-based learning. Blended learning is becoming a very popular form of training (Davis \& Fill, 2007; Welsh, et al., 2003). It aims to address learners' inability to completely adapt to online courses as it provides learners and instructors the benefit and the convenience of online courses without the loss of the conventional face-to-face method. Condie and Livingston (2007) noted that blended learning is designed to complement traditional teaching and learning rather than replace it.

Most e-learning institutions are characteristically asynchronous (Welsh, et al., 2003) enabling people to learn anytime and anywhere, thus learners and instructors do not have person-to-person simultaneous interaction during teaching or learning processes (Maglogiannis \& Karpouzis, 2007). The pre-recorded learning materials or processes can be delivered through an open network or the WWW, private intranets, or home computer-based study applications, and communicated through emails and online messaging. Asynchronous interaction is two way communication that happens with time delay whether it is affected by the communication tools or by choice for participants' convenience in order to deal with time zone differences. The interaction takes place over a period of time and it is typically in the form of discussion groups. Participants are able to post messages in a discussion group. Others can reply over the following days, weeks or not at the same time. Participation is very important in a discussion group, thus it continuously requires stimulation and encouragement so that people are willing to participate.

Maglogiannis and Karpouzis (2007) developed a typical e-learning integrated architecture for military training purposes which was implemented in the School of Research and Informatics for Officers of the Greek Army (Figure 6). Figure 6 shows that e-learning systems involve learners, trainers, support personnel, and systems and 
content developers. Each of them has access to technology including hardware, software, servers, and a network connection or the Internet. The systems provide a rich, collaborative learning environment to end users, and are accessible from physically dispersed locations, 24 hours a day, and 7 days a week.

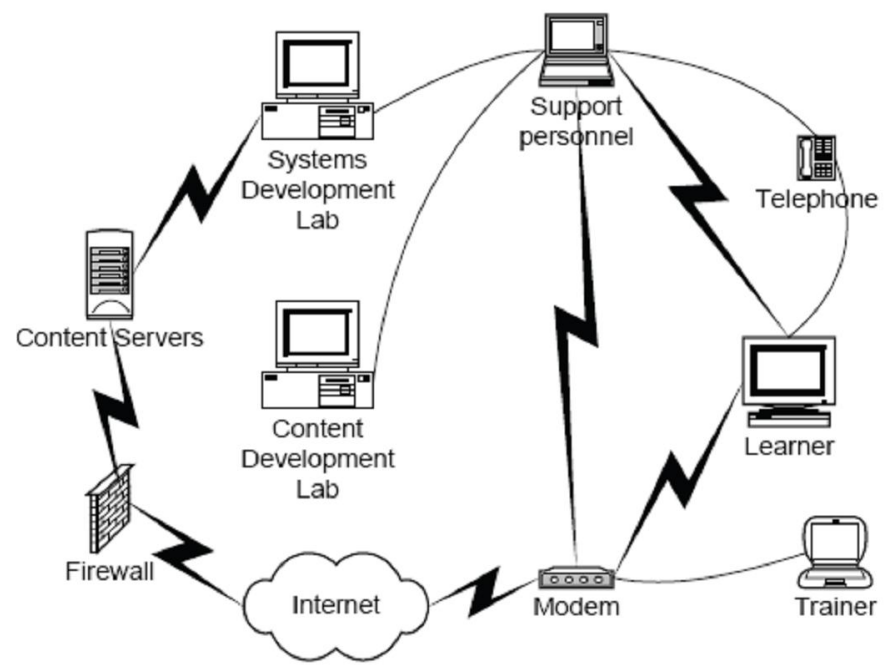

Figure 6: E-learning integrated architecture

(Maglogiannis \& Karpouzis, 2007, p. 27)

\subsubsection{Why do we need organisational e-learning?}

Aydin and Tasci (2005) confirmed that OES is one of the main innovations that are increasingly appearing in corporate settings. Haney (2002) also added that OES is becoming commonplace for instructional designers, human performance technologists, trainers, and human resources professionals, as well as end-user learners. Previous studies identified a number of reasons why organisations implement OES.

Welsh et al. (2003) noted that e-learning has flourished in organisational training as it enables consistent training delivery via the Internet across multiple locations. Most organisations continuously develop their human resources through either training or other higher academic education. It is becoming more critical for businesses to have an experienced and highly skilled workforce in order to survive in the global market. However, large organisations with distributed branch offices are potentially faced with inconsistency and communication problems in delivering training through traditional learning methods across branches. Remote offices often have their own culture of adapting the learning. Moreover, the lessons delivered may be inconsistent with the original lessons as they may be poorly communicated from the central office to branch 
offices. OES which naturally use asynchronous means are designed to enable participants to share standardised information throughout the entire organisation at their own pace.

OES are flexible systems offering powerful tools for organisational knowledge management which can change quickly to keep pace with a shifting business environment (Chan \& Ngai, 2007; Wild, Griggs, \& Downing, 2002). Therefore, it allows organisations to meet their critical and strategic requirements for flexible, welltrained employees in order to survive global competition. The systems allow learners to access educational resources not only from inside the organisation but also from outside the organisation on a global and immediate basis (Sharma \& Mishra, 2007; Wild, Griggs, \& Downing, 2002). This offers opportunities to be connected in a global, crosscultural, and collaborative learning environment which may expand the learners' mindset and enhance their experiences.

E-learning can be delivered to a large number of learners across dispersed areas at the same time (Chan \& Ngai, 2007; Lopez, 2005; Stokes, et al., 2004; Welsh, et al., 2003). This reduces the cycle of delivery time, as the training can be given to a large number of learners across multiple boundaries compared to traditional learning methods which can only train limited numbers of learners in a particular place (a classroom) and particular time. To train a large number of learners through traditional methods requires substantial resources including considerable time, significant effort, and a lot of space. It takes a longer time to deliver in-class training and numerous classrooms are required to accommodate a large number of students. The availability of instructors and classrooms will also affect the delivery cycle time. A large organisation usually requires an extremely broad range of training for a great number of employees. It is critical to assure that knowledge and vital information can be disseminated swiftly throughout the organisation.

OES save resources and diminish organisational barriers by providing online education across the boundaries of time and space. The network technology enables organisations to provide training and information sharing for a large number of learners across those barriers. OES minimise distribution costs for just-in-time training at any time and any location. Welsh et al. (2003) and Lopez (2005) noted that cost savings involve reduced 
travel expenditure, saved time, and reduced classroom allocation. The systems can be deployed to the entire organisation at once and do not require booking auditoriums, making travel reservations, and providing accommodation for large numbers of trainers and trainees (Chan \& Ngai, 2007; Welsh et al., 2003). Welsh et al. (2003) mentioned that OES implementation in Dow Chemical had saved approximately $\$ 30$ million. Roughly \$20 million of that was as a result of a reduction of time employees spent on training, while $\$ 10$ million was saved due to a decrease in administrative time, cost of classroom facilities, facilitators' fees, and the cost of printed materials.

OES enables employees to improve their skills and job-related expertise through eleaning systems without having to leave their job. It offers customised training that takes less time because there is no need to travel to the training site or make time for the training within the employee's regular workday. It enables participants to access information with minimal loss of their productivity (Chan \& Ngai, 2007; Sharma \& Mishra, 2007; Stokes, et al., 2004; Welsh, et al., 2003). Individual learners who cannot undertake the conventional training method of attending classes can use OES to learn from their homes or offices by using the Internet or an organisation's intranet (Bell, Martin, \& Clarke, 2004; Chan \& Ngai, 2007). Maglogiannis and Karpouzis (2007) found that distance learning for military training meant that people could attend the lessons without leaving their positions, and they can communicate with their colleagues located in distant units.

OES is a flexible independent delivery platform that enables the delivery of education from instructors to learners and is accessible through any computer with a simple browser interface (Sharma \& Mishra, 2007; Welsh, et al., 2003). Individual learners and instructors can interact with each other efficiently and effectively through asynchronous and/or synchronous communication channels (Chan \& Ngai, 2007; Sharma \& Mishra, 2007). Participants may choose to communicate, participate, and collaborate at their own time and pace which can be done by exchanging printed or electronic media supported by ICT.

OES facilitates the creation of in-depth trackable training. OES offers the capability to track learners' activities and mastery of the materials as the systems can automatically create, update, revise (Sharma \& Mishra, 2007), track, and store extensive course 
materials through low cost off-the shelf software (Maglogiannis \& Karpouzis, 2007; Unneberg, 2007; Welsh, et al., 2003). Compared to conventional learning methods these capabilities are helpful when such training requires compliance as managing and maintaining training for a large number of learners across dispersed areas could be cumbersome. It is difficult to deliver course materials to all learners and track all learners' activities in remote places using conventional methods and they require significant time, effort, and resources.

OES can be used to efficiently manage the continually increasing amount of information delivered to learners. Welsh et al. (2003) noted that including a vast amount of information often makes training ineffective as learners may be overwhelmed by it in classroom-based training. Thus, blended learning systems can be employed to overcome this issue as the delivery of lessons can be done in three different ways: in-class training (only for the most interactive lessons), asynchronous, and synchronous. The information can be delivered over a longer period of time and learners can obtain the information they need through several methods and choose which are best suited to them. Accordingly, this may reduce learner turnover and improve retention. Table 5 presents a summary of OES benefits according to the previous literature.

Table 5. Summary of e-learning benefits

\begin{tabular}{|l|l|}
\hline \multicolumn{1}{|c|}{ Benefits of e-learning } & \multicolumn{1}{|c|}{ Study } \\
\hline Reduced time & Unneberg, 2007 \\
\hline Reduced cost creating e-learning & Unneberg, 2007 \\
\hline Enabling SME (subject matter expert) as the primary source for content development & Unneberg, 2007 \\
\hline Having easy-to-use software that reduces the barrier of creating content & Unneberg, 2007 \\
\hline Providing consistent worldwide training & Welsh et al., 2003 \\
\hline Reducing delivery cycle time & Welsh et al., 2003 \\
\hline Increasing learner convenience & Welsh et al., 2003 \\
\hline Reducing information overload & Welsh et al., 2003 \\
\hline Improving tracking & Welsh et al., 2003 \\
\hline Creating lower expenses & Welsh et al., 2003 \\
\hline Enabling employees to take training in a timely manner & Lopez, 2005; Unneberg, 2007 \\
\hline Increasing access to instruction and resources for employees & Chan \& Ngai, 2007 \\
\hline $\begin{array}{l}\text { Providing access to all levels of education to individuals that are geographically } \\
\text { dispersed }\end{array}$ & $\begin{array}{l}\text { Bell, Martin, \& Clarke, 2004; Chan \& Ngai, 2007; } \\
\text { Lopez, 2005; Maglogiannis \& Karpouzis, 2007 }\end{array}$ \\
\hline $\begin{array}{l}\text { Delivering instruction or teaching courses in remote locations that are difficult for } \\
\text { trainers/instructors to access }\end{array}$ & $\begin{array}{l}\text { Bell, Martin, \& Clarke, 2004; Lopez, 2005; } \\
\text { Maglogiannis \& Karpouzis, 2007 }\end{array}$ \\
\hline $\begin{array}{l}\text { Providing access to individuals whose absence from their positions may cause } \\
\text { additional problems in the operation of their units }\end{array}$ & Maglogiannis \& Karpouzis, 2007 \\
\hline $\begin{array}{l}\text { Frequently delivering large scale training and education, at high volume and low cost } \\
\text { Developing strong employer brands nationally and internationally }\end{array}$ & $\begin{array}{l}\text { Bell, Martin, \& Clarke, 2004; Unneberg, 2007; } \\
\text { Welsh, et al., 2003 }\end{array}$ \\
\hline $\begin{array}{l}\text { Encouraging the development of generic business skills and knowledge through } \\
\text { business universities }\end{array}$ & Bell, Martin, \& Clarke, 2004; Chan \& Ngai, 2007 \\
\hline
\end{tabular}




\subsubsection{What are the downsides of e-learning implementation?}

Unneberg (2007) identified several challenges in building training programmes in a large organisation including: developing training programmes that can address the business issues within the organisation; assuring the consistency of the training; ensuring the training is approved and delivered throughout the entire enterprise in a timely fashion; and ensuring reasonable costs development. Welsh et al. (2003) identified potential drawbacks of using e-learning including: requiring a considerable amount of resources; lacking interaction; having a static and non-interactive mindset; and requiring significant effort and planning. Ali and Magalhaes (2008) also identified the most significant barriers to e-learning implementation in the West including cost, time, technology, and resistance to change. They also identified other key barriers to OES adoption: lack of appropriate content related to specific needs; language barriers; difficulties in measuring e-learning effectiveness; lack of strategic planning and direction particularly when the system is not aligned with business objectives; lack of elearning awareness; lack of incentives; and lack of management support.

It seems that cost is the foremost barrier especially for initiating and implementing elearning. The initiation of e-learning systems requires considerable up-front costs (Ali \& Magalhaes, 2008; Bell, Martin, \& Clarke, 2004; Unneberg, 2007; Welsh, et al., 2003). The costs required include investment for developing IT infrastructure and human resources; designing and building the actual courses, and providing hardware and software to allow users to access the e-learning systems. Additionally, Ali and Magalhaes (2008) noted that technology is integral, expensive, unpredictable, and can become outdated. Thus, it is necessary to provide additional ongoing costs for upgrading and maintaining the systems as well. Bell, Martin, and Clarke (2004) also noted that learners would be eager to use e-learning if they could afford the technology as individuals.

Nichols (2007) listed the main barriers to sustainable e-learning implementation as poor strategic ownership; insufficient support from senior management; the culture not being ready for innovation; and e-learning misconceptions. Moreover, e-learning systems might be disadvantaged by the existing institutional environment such as inability/unwillingness to change and poor/incompetent professional development. Nichols (2007) confirmed that sustainable embedded e-learning can be achieved by 
proactive activity (permitting forward thinking and further planning); scalability (deploying e-learning rapidly across new programmes so that new approaches can be readily adopted); and self-perpetuation (making e-learning an established part of operations).

According to Ali and Magalhaes (2008) time is a critical barrier amongst the top three barriers against implementing e-learning in the workplace, including lack of time for identifying the main challenges of e-learning, lack of time for conducting training, and lack of time for learning. Time is a significant barrier to e-learning due to increasing workloads (Ali \& Magalhaes, 2008; Nichols, 2007). Indeed, most employees have demanding responsibilities and duties, so they may have inadequate time to devote to workplace learning with all their work interruptions and insufficient time to develop and maintain e-learning. Thus, the time commitment is difficult to manage (Ali \& Magalhaes, 2008; Aydin \& Tasci, 2005). Learners may have difficulty concentrating on learning and allocating time for studying in either their homes or offices.

One of the key barriers for e-learning adoption and implementation is technical issues (Ali \& Magalhaes, 2008; Aydin \& Tasci, 2005; Condie \& Livingston, 2007) including: system crashes; bandwidth and infrastructure upgrading; accessibility; usability; availability of technical support; perceived difficulties in using such a system (users are required to be proficient with online skills); fear of technology; and perceived difficulties in performing online procedures (using passwords or dealing with permissions). Ali and Magalhaes (2008) also noted problems dealing with adjustment, integration, compatibility, and capability. Implementing new technology requires adjustment from both sides: the organisation and the learners. Effective e-learning implementation can be accomplished by integrating e-learning technology with the existing systems, considering software and hardware compatibilities, ensuring appropriate capacity to run e-learning systems, and enhancing employees' technological competency (ability to use, upgrade, and customise the technology). The inadequacy of technical support and/or qualified e-learning suppliers that do not provide technical support will also make an organisation reluctant to use e-learning as a training solution. 
According to Sadik (2007) three main reasons that faculty resist using e-learning materials in university teaching are lack of knowledge, lack of technological skills, and negative attitudes towards technology usage. He identified variables that affect the proper use of technology in university teaching: years of teaching, level of computer literacy, degree held, academic profession, and training received. Mutula and Brakel (2007) specified that an ICT skills shortage remains the greatest obstruction not only in developing countries but also in developed countries. They found that Europe had advocated resources to implement widespread Internet access; train teachers in Internet literacy; reskill the workforce to be able to operate in an increasingly ICT-oriented business environment; and put in place initiatives such as e-learning and online public services.

Maglogiannis and Karpouzis (2007) claimed that learners who are not familiar with new technology tend to have lower participation and lower motivation in the learning process. Condie and Livingston (2007) confirmed that instructors who have low confidence and little understanding of using ICT to promote learning hamper e-learning adoption. Furthermore, those who are sceptical about the benefits of ICT use in their subject and reluctant to relinquish the role of expert transmitter of knowledge hinder elearning adoption. Instructors must be proficient in ICT, especially in the area of adopting OES.

White (2007) confirmed that an institution's internal culture, structure, system, and climate may affect OES implementation. The most visible cultural impediment to OES implementation is internal resistance to using technology (Ali \& Magalhaes, 2008; Aydin \& Tasci, 2005; Condie \& Livingston, 2007; Nichols, 2007; Sadik, 2007). Ali and Magalhaes (2008) confirmed that if the intended users refused to accept the systems, OES is considered unsuccessful whether it was well-designed with the specifications of the job skills or well-aligned with the business goals. Aydin and Tasci (2005) noted that people who are resistant to technology and technophobic would cause an organisation to suffer from reduced work productivity, lower job satisfaction, and decreased profits and efficiency. Ali and Magalhaes (2008) affirmed that it is critical to assure that OES bringing new solutions and practices are embedded in the entire organisation's culture. 
Nichols (2007) notified that managerial challenges may involve recognising differences which occur in the organisation's climate including variations on total assets; management style disparities; variations in motivation levels to innovate teaching; and variations in drivers to change teaching (centralised or decentralised teaching policies). White (2007) noted that to disseminate the notion of e-learning, policy makers are responsible for making a systematic holistic approach of coordination between all stakeholders (managers, administrators, providers and learners) and fostering alignment between technology and the organisation. A clear understanding of the alignment between e-learning objectives, the role of managers, their actions, and approaches that should be adjusted to the organisational context is essential.

Nichols (2007) identified general concerns about OES adoption among many employees: resistance to change; poor leadership; information technologies' selfefficacy; lack of effective staff development; and drawn-out implementation. When employees are expected to embrace innovations besides their existing demanding responsibilities, they may become resistant to adopting new systems. Employees may also feel that the systems create more trouble than benefits. They may be unable to use the technology attached to the systems or the systems may not be applicable to their specific tasks and projects (Ali \& Magalhaes, 2008). In this case, it is likely that they will feel uncomfortable with the new technology and object to using the systems, which is also affirmed by Aydin and Tasci (2005). Welsh et al. (2003) added that change management concerns include how to prepare users and training department staff for the change, and how to gain and sustain senior management support for the initiative.

Welsh et al. (2003) explained that the use of electronic technology to deliver materials in an online environment is mostly in the form of static and non-interactive materials which may lead to a mindset that training or learning is merely electronically-encoded information. There is a possibility that top management become preoccupied with the ability to disseminate information and forget that training involves more than information provision. It is important to recognise that e-learning not only entails providing electronically-encoded information through the network but also involves learning practices, providing interactive feedback and guidance. 
OES may appear less attractive and less useful if it does not include interaction among learners (Bell, Martin, \& Clarke, 2004; Welsh et al., 2003). Peer-to-peer interaction supported by network technology is critical to perform a sustainable OES implementation, though in some cases it may require intensive resources. However, in most circumstances the culture of online communication does not yet exist. Stokes et al. (2004) confirmed that the culture needs to be altered and familiar with ICT or online media throughout an organisation which intends to deploy collaborative online learning. Some learners may be able to access the Internet and use email to communicate, while others may have insufficient experience of using other interactive communication methods. Regardless of the advancement of technology to deliver information, most learners still rely on conventional means in accessing literature. OES require learners to have the ability to use online means to interact within the environment.

Besides the complex and confusing technology, Bell, Martin, and Clarke (2004) confirmed that fear of security and identity problems with the Internet may cause a loss of trust in the integrity of e-learning. Learners will be more likely to use the systems if organisations maintain strong control over intellectual capital.

\subsection{Critical success factors for developing and implementing OES}

Welsh et al. (2003) noted that e-learning implementation requires considerable planning and effort. In this case, e-learning models can be applied to prepare for the development of effective OES. They can be used as a starting point by listing critical aspects that need to be considered in developing OES. DeLone and McLean (2004) introduced an Information Systems Success Model to evaluate the success of e-commerce systems which can be adapted to the measurement challenges of the new e-commerce world. Their model was enhanced by Holsapple and Lee-Post (2006) particularly to define, assess, and promote e-learning systems by developing an E-learning Success Model. They confirmed that this model's application may support training developers to design, develop, and deliver e-learning initiatives. Figure 7 shows the E-Learning Success Model which consisted of three e-learning development stages: system design, system delivery, and system outcome. The model defines e-learning success in six dimensions: system quality, information quality, service quality, use, user satisfaction, and net benefits. 
Wild, Griggs, and Downing (2002) proposed an e-learning value chain comprising four elements of the e-learning planning process: assessing and preparing organisational readiness; determining the appropriate content; determining the presentation modes; and implementing e-learning. Welsh et al. (2003) added three areas that should be considered in planning e-learning implementation: training design; IT infrastructure; and change management.

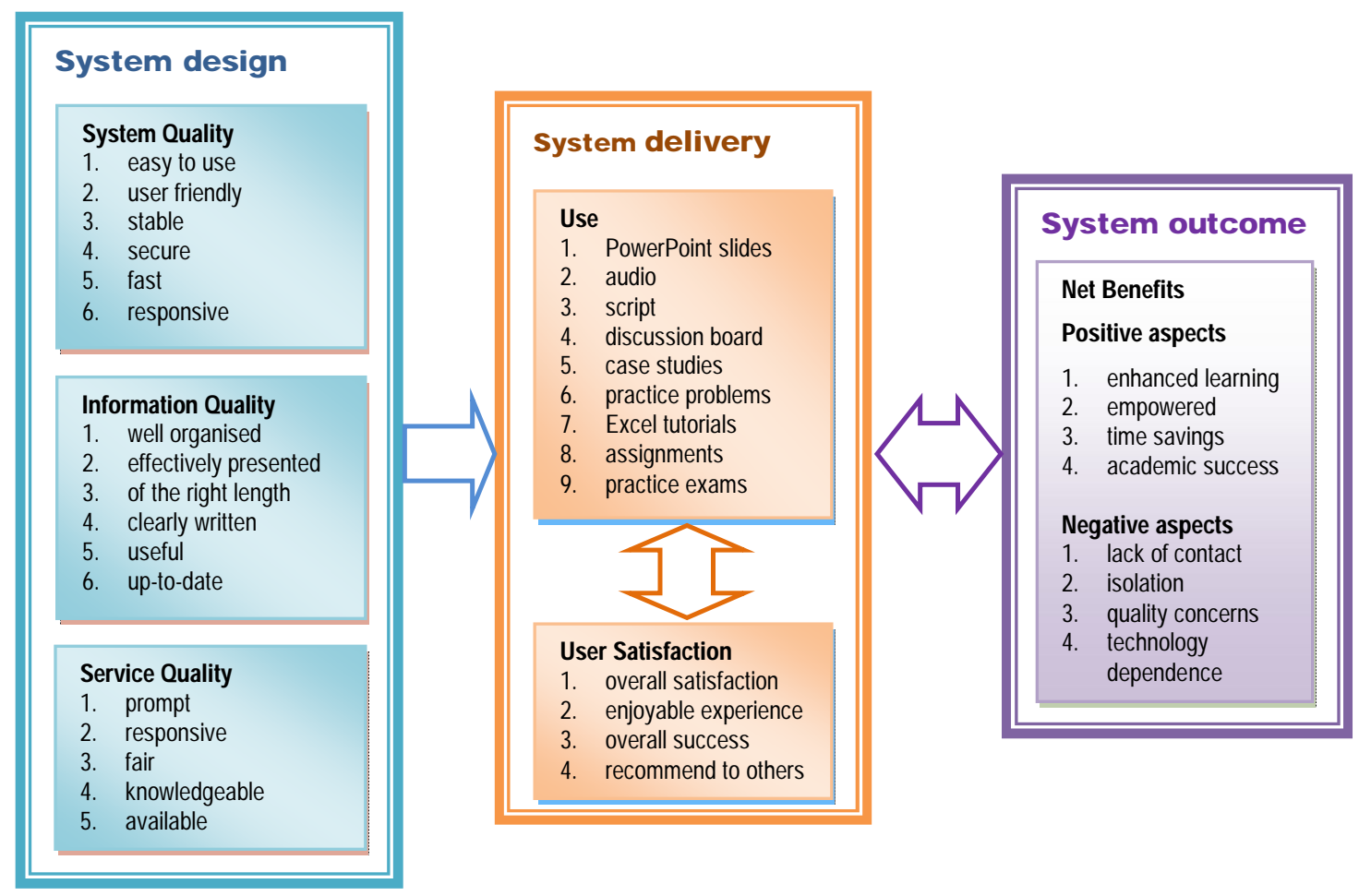

Figure 7: E-Learning Success Model developed (Holsapple \& Lee-Post, 2006, p. 71)

(Holsapple \& Lee-Post, 2006, p. 71):

- The arrows represent the interdependences within the three stages of success assessment.

- A single arrow indicates success in system design which is essential to the success of system delivery, which, in turn, affects the success of system outcome.

- A double arrow linking system delivery and outcome stages indicates that the successful system outcome has an impact on the success of subsequent system delivery.

Cook and Dupras (2004) proposed 10 steps in three major phases of developing effective e-learning: the preparatory, development, and implementation and maintenance phases. Phase 1 involves four parts: (1) performing a needs analysis and specifying goals and objectives; (2) determining technical resources and needs; (3) evaluating commercial software and using it if it fully meets the needs; and (4) securing a commitment from all participants, and identifying and addressing potential barriers to implementation. Phase 2 also has four parts: (5) developing content in close coordination with website design; (6) encouraging active learning - self assessment, 
reflection, self-directed learning, problem-based learning, learning interaction, and feedback; (7) facilitating and planning to encourage use; and (8) evaluating both learners and courses. Phase 3 comprises: (9) conducting a pilot study of the website before full implementation; and (10) planning to monitor online communication and maintaining the site.

Welsh et al. (2003) identified four features that need to be considered in technologydelivered training: effectiveness (does e-learning stimulate learning?); efficiency (to what extent do these programmes use resources efficiently?); attrition (how many users drop out of such training?); and appeal (how do the learners react to the training?).

Nichols (2007) identified six significant factors for successful e-learning diffusion: centres of power; strategic ownership and e-learning acceptance; the level of each institution's readiness for change interventions; alignment between e-learning activity, policy, and systems; professional development; and dynamics of change of different sized institutions (small, medium, and large). He also identified insignificant factors: $e$ learning perceived as expensive; team course development; adopting additional quality assurance criteria; and having a single focus department.

Nichols (2007) found that organisations that make e-learning part of their daily activities achieve sustainable e-learning implementation, while those that perceive elearning as outside their core activities cannot achieve that. The former groups are more likely to be ready for transformation and to provide a platform for further innovation, whilst the latter groups need to address the obstructions to sustainable adoption.

Sharma and Mishra (2007) identified six key dimensions of effective e-learning design: connectivity (global access to information); interactivity (spontaneous assessment); motivation (enhanced learning); flexibility (anytime/anyplace learning); collaboration (through online discussion tools); and extended opportunities (reinforced learning). They formulated guidelines for effective learning involving developing learners' skills and knowledge; engaging learners in the learning processes; developing independent learning skills; and motivating learners. They identified six factors to consider when planning online distance learning programmes in higher education: vision and plan; curriculum; staff training and support; student services; student training and support; 
and copyright and intellectual property. They also revealed other relevant factors such as faculty support; technology selection for online programmes; and collaboration among different components of the systems.

Bohl and Scheer (2007) noted that in order to individualise services and identify target learner segments, integration (between learners-the whole value added process; elearning providers-learners; and learner-learner) is a success factor in academic education products. They noted that high quality education involves up-to-date content; individuality of learning relations; just-in-time learning content provision; method knowledge richness; and integrated educational offers and programme provision. Davis and Fill (2007) added: active involvement of senior managers; the whole curriculum approach; funding; support at the point of need; and collaboration.

These critical success factors can be taken into a consideration as measurement factors while investigating the readiness of an organisation to implement e-learning.

\subsection{Assessing organisational readiness for OES}

According to the Economist Intelligence Unit Limited \& IBM Corporation (EIU \& IBM, 2003) Indonesia scored 3.67 out of 10 and ranked 53rd out of 60 in e-learning readiness. Indonesia ranked 68th out of 70 countries in e-readiness with an overall score of 3.59 out of 10 (EIU \& IBM, 2008). There are several questions that should be asked during preparation for e-learning. Table 6 shows the key questions to answer when assessing organisational readiness for knowledge management (KM). It is possible that some of these dimensions could be adapted for assessing organisational readiness for OES.

Pillay, Irving, and Tones (2007) noted that online learning readiness involves the ability to manage time, to adapt to self-directed learning, to boost motivation, and to understand personal learning styles and experiences. Haney (2002) suggested that assessing organisational readiness involve a set of skills or abilities to harmonise change management, examine multiple aspects of situations, conduct cost-benefit analysis, and identify political problems. 
Table 6. Critical components in assessing organisational readiness

\begin{tabular}{|c|c|c|}
\hline Components & Questions & Questions adapted for e-learning \\
\hline Infrastructure & $\begin{array}{l}\text { Does a knowledge management infrastructure exist? } \\
\text { Does the organisation already support a knowledge } \\
\text { management }(\mathrm{KM}) \text { infrastructure? }\end{array}$ & $\begin{array}{l}\text { Does e-learning infrastructure exist? } \\
\text { Does the organisation already support e-learning } \\
\text { infrastructure? }\end{array}$ \\
\hline $\begin{array}{l}\text { Knowledge } \\
\text { editor }\end{array}$ & $\begin{array}{l}\text { Is the organisation willing to invest in a knowledge editor? } \\
\text { What may be incorporated into the e-learning } \\
\text { environment? } \\
\text { Is the organisation willing to create functional units in } \\
\text { place solely for the purpose of overseeing the fusion of } \\
\text { KM into e-learning courseware and vice versa? }\end{array}$ & $\begin{array}{l}\text { Is the organisation willing to invest in e-learning systems? } \\
\text { What may be incorporated into the e-learning environment? } \\
\text { Is the organisation willing to create functional units solely } \\
\text { for the purpose of overseeing the fusion of traditional } \\
\text { learning into e-learning courseware and vice versa? }\end{array}$ \\
\hline $\begin{array}{l}\text { Organisational } \\
\text { culture }\end{array}$ & $\begin{array}{l}\text { Does the existing culture encourage and promote } \\
\text { knowledge sharing? } \\
\text { Is the organisation willing to invest in a paradigm shift } \\
\text { from knowledge hoarding to knowledge sharing? } \\
\text { Have clearly articulated policies been established to } \\
\text { explain the benefits of e-learning and its ties to KM? }\end{array}$ & $\begin{array}{l}\text { Does the existing culture encourage and promote } \\
\text { knowledge sharing? } \\
\text { Is the organisation willing to invest in a paradigm shift from } \\
\text { knowledge hoarding to knowledge sharing? } \\
\text { Have clearly articulated policies been established to explain } \\
\text { the benefits of e-learning? }\end{array}$ \\
\hline $\begin{array}{l}\text { Employee } \\
\text { attitudes }\end{array}$ & $\begin{array}{l}\text { Do employees accept the notion of sharing knowledge? } \\
\text { Have employees been persuaded to share knowledge, } \\
\text { rather than keep it to themselves in the tradition of } \\
\text { "knowledge is power"? } \\
\text { Are they willing to commit to a policy of self-study? }\end{array}$ & $\begin{array}{l}\text { Do employees accept the concept of e-learning? } \\
\text { Have employees been persuaded to learn using e-learning } \\
\text { systems, rather than using conventional learning? } \\
\text { Are they willing to commit to a policy of self-study? }\end{array}$ \\
\hline $\begin{array}{l}\text { Knowledge } \\
\text { needs }\end{array}$ & Have the strategic knowledge needs been identified? & $\begin{array}{l}\text { Have the strategic e-learning systems needs been } \\
\text { identified? }\end{array}$ \\
\hline $\begin{array}{l}\text { Computer } \\
\text { usage }\end{array}$ & Are employees computer literate? & Are employees computer literate? \\
\hline $\begin{array}{l}\text { Technology } \\
\text { requirements }\end{array}$ & $\begin{array}{l}\text { Is the organisation sufficiently "wired"? } \\
\text { Does the organisation's technology infrastructure support } \\
\text { the bandwidth, multimedia, ISPs, etc, necessary for } \\
\text { creating an e-learning/KM environment? }\end{array}$ & $\begin{array}{l}\text { Is the organisation sufficiently "wired"? } \\
\text { Does the organisation's technology infrastructure support } \\
\text { the bandwidth, multimedia, ISPs, etc, necessary for } \\
\text { creating e-learning? }\end{array}$ \\
\hline
\end{tabular}

Summarised from Wild, Griggs, \& Downing (2002)

Previous studies discussed various aspects that can be used to measure organisational readiness for e-learning implementation. A number of instruments have been developed to assess e-learning readiness. These instruments from previous studies were examined to provide a robust foundation for this research. Nine instruments were selected due to their relevance to the topic being studied. They were chosen as they represent both developed and developing country contexts. They also provide various aspects of elearning in order to conceptualise the domains that directly affect readiness to successfully develop and implement e-learning. Diverse e-learning dimensions were identified (Table 7) from the nine studies. Of these nine instruments only five were selected due to their relevance to this study (Table 8) and used to develop the COERI. 
Table 7. Summary of e-learning readiness dimensions from nine studies

\begin{tabular}{|c|c|c|c|c|c|c|c|c|c|c|}
\hline No & Study & $\begin{array}{c}\text { Chapnick, } \\
2000\end{array}$ & $\begin{array}{l}\text { EIU \& } \\
\text { IBM, } \\
2003\end{array}$ & $\begin{array}{c}\text { Watkins, } \\
\text { Leigh, \& } \\
\text { Triner, } \\
2004 \\
\end{array}$ & $\begin{array}{c}\text { Abas, } \\
\text { Kaur, \& } \\
\text { Harun, } \\
2004 \\
\end{array}$ & $\begin{array}{l}\text { Smith, } \\
2005\end{array}$ & $\begin{array}{l}\text { Aydin \& } \\
\text { Tasci, } \\
2005\end{array}$ & $\begin{array}{l}\text { Karmakar } \\
\text { \& Wahid, } \\
2006\end{array}$ & $\begin{array}{l}\text { Sadik, } \\
2007\end{array}$ & $\begin{array}{c}\text { Pillay, } \\
\text { Irving, \& } \\
\text { Tones, } \\
2007 \\
\end{array}$ \\
\hline 1 & Attitude & & & & & & & & $\sqrt{ }$ & $\sqrt{1}$ \\
\hline 2 & Innovation & & & & & & $\sqrt{ }$ & & & \\
\hline 3 & Psychological & $\sqrt{ }$ & & & & & & & & \\
\hline 4 & People & & & & & & $\sqrt{ }$ & & & \\
\hline 5 & Self-development & & & & & & $\sqrt{ }$ & & & \\
\hline 6 & Sociological & $\sqrt{ }$ & & & & & & & & \\
\hline 7 & Capability & & $\sqrt{ }$ & & & & & & & \\
\hline 8 & Technology & & & & & & $\sqrt{ }$ & & & \\
\hline 9 & Technological skills & $\sqrt{ }$ & & & & & & & & $\sqrt{ }$ \\
\hline 10 & Technical & & & & $\sqrt{ }$ & & & & & \\
\hline 11 & Technological competencies & & & & & & & & $\sqrt{ }$ & \\
\hline 12 & Computer self-efficacy & & & & & & & & & $\sqrt{ }$ \\
\hline 13 & Pedagogical competencies & & & & & & & & $\sqrt{ }$ & \\
\hline 14 & Online skills and relationships & & & $\sqrt{ }$ & & & & & & \\
\hline 15 & Equipment & $\sqrt{ }$ & & & & & & & & \\
\hline 16 & Online audio/video & & & $\sqrt{ }$ & & & & & & \\
\hline 17 & Internet discussion & & & $\sqrt{ }$ & & & & & & \\
\hline 18 & Content & $\sqrt{ }$ & $\sqrt{ }$ & & $\sqrt{ }$ & & & $\sqrt{ }$ & & \\
\hline 19 & Training process & & & & & & & $\sqrt{ }$ & & \\
\hline 20 & Culture & & $\sqrt{ }$ & & $\sqrt{ }$ & & & $\sqrt{ }$ & & \\
\hline 21 & Financial & $\sqrt{ }$ & & & $\sqrt{ }$ & & & $\sqrt{ }$ & & \\
\hline 22 & Human resource & $\sqrt{ }$ & & & & & & & & \\
\hline 23 & Personnel & & & & $\sqrt{ }$ & & & & & \\
\hline 24 & Management & & & & $\sqrt{ }$ & & & & & \\
\hline 25 & Learner & & & & $\sqrt{ }$ & & & & & \\
\hline 26 & Environmental & $\sqrt{ }$ & & & $\sqrt{ }$ & & & & & \\
\hline 27 & Connectivity & & $\sqrt{ }$ & & & & & & & \\
\hline 28 & Technology access & & & $\sqrt{ }$ & & & & & & \\
\hline 29 & Experience & & & & & & & & $\sqrt{ }$ & \\
\hline 30 & Learner preferences & & & & & $\sqrt{ }$ & & & & $\sqrt{ }$ \\
\hline 31 & Self-management learning & & & & & $\sqrt{ }$ & & & & \\
\hline 32 & Motivation & & & $\sqrt{ }$ & & & & & & \\
\hline 33 & Importance to your success & & & $\sqrt{ }$ & & & & & & \\
\hline
\end{tabular}


Table 8. Summary of five instruments chosen

\begin{tabular}{|c|c|c|c|c|c|}
\hline Author(s) & Abas, Kaur, \& Harun (2004) & Aydin \& Tasci (2005) & Pillay, Irving, \& Tones (2007) & Sadik (2007) & Watkins, Leigh, \& Triner (2004) \\
\hline Instrument(s) & E-learning Readiness (ELR) & E-Learning Readiness Survey (eLRS) & $\begin{array}{l}\text { Tertiary students' readiness for online } \\
\text { learning (TSROL) }\end{array}$ & $\begin{array}{l}\text { Individual readiness to develop and } \\
\text { implement e-learning (IRDI-EL) }\end{array}$ & $\begin{array}{l}\text { E-Learning Readiness Self- } \\
\text { Assessment (ELRSA) }\end{array}$ \\
\hline Purpose & $\begin{array}{l}\text { To assess e-learning readiness in } \\
\text { Malaysia }\end{array}$ & To assess e-learning readiness in Turkey & $\begin{array}{l}\text { To assess tertiary students' readiness } \\
\text { for online learning }\end{array}$ & $\begin{array}{l}\text { To assess individual readiness in } \\
\text { developing and implementing e- } \\
\text { learning }\end{array}$ & To assess learners' readiness \\
\hline $\begin{array}{l}\text { Target } \\
\text { respondents }\end{array}$ & $\begin{array}{l}\text { Policy makers, providers, enablers, and } \\
\text { receivers. }\end{array}$ & Policy makers & Receivers & Enablers & $\begin{array}{l}\text { Receivers with no prior e-learning } \\
\text { experience }\end{array}$ \\
\hline Research context & Malaysia & Turkey & Australia & Egypt & United States of America \\
\hline Subscales & $\begin{array}{l}\text { (1) learner; (2) management; (3) content; } \\
\text { (4) personnel; (5) technical; (6) financial; } \\
\text { (7) environmental; and (8) cultural }\end{array}$ & $\begin{array}{l}\text { (1) Ppeople; (2) self-development; (3) } \\
\text { technology; and (4) innovation }\end{array}$ & $\begin{array}{l}\text { (1) technical skills; (2) computer self- } \\
\text { efficacy; (3) learner preferences; and } \\
\text { (4) attitude towards computers }\end{array}$ & $\begin{array}{l}\text { (1) competencies; (2) experience; } \\
\text { and (3) attitudes toward the } \\
\text { development and implementation of } \\
\text { e-learning in university teaching }\end{array}$ & $\begin{array}{l}\text { (1) technology access; (2) online skills } \\
\text { and relationships; (3) motivation; (4) } \\
\text { online audio/video; (5) Internet } \\
\text { discussions; and (6) importance to your } \\
\text { success }\end{array}$ \\
\hline $\begin{array}{l}\text { Number of } \\
\text { questions }\end{array}$ & $\begin{array}{l}\text { A two section survey: Section A contains } \\
\text { demographic variables; Section B contains } \\
\text { readiness assessment } \\
39 \text { questions for policy makers } \\
42 \text { questions for providers } \\
71 \text { questions for enablers } \\
62 \text { questions for receivers }\end{array}$ & $\begin{array}{l}\text { A two section survey: } \\
\text { Section } 1 \text { contains } 10 \text { items of } \\
\text { demographic characteristics } \\
\text { Section } 2 \text { contains } 30 \text { items of readiness } \\
\text { assessment (a five-point Likert scale) }\end{array}$ & 20 items - a seven-point Likert scale & $\begin{array}{l}66 \text { items : } \\
19 \text { items - a four-point Likert scale } \\
3 \text { items - a two-point Likert scale } \\
4 \text { items - a three-point Likert scale } \\
8 \text { items - a four-point Likert } \\
32 \text { items - a five point Likert scale }\end{array}$ & 27 items - a five-point Likert scale \\
\hline Sample & $\begin{array}{l}5779 \text { target respondents: } \\
102 \text { policy makers from private and } \\
\text { government sectors } \\
75 \text { providers (education, training, R\&D, } \\
\text { and ICT) } \\
977 \text { enablers (private and government } \\
\text { higher educational institutions) } \\
4625 \text { receivers (learners, trainees from } \\
\text { private and government higher education } \\
\text { institutions, and participants attending in- } \\
\text { service training programmes) } \\
6615 \text { responses received: } \\
\text { Online survey were emailed to } 3500 \\
\text { respondents, } 836 \text { responses completed } \\
9950 \text { printed copies were sent, } 5779 \\
\text { responses completed }\end{array}$ & $\begin{array}{l}\text { The directors of the human resources } \\
\text { departments in the top } 100 \text { companies of } \\
\text { Turkey } \\
50 \text { responses received }\end{array}$ & $\begin{array}{l}480 \text { target respondents: students in } \\
\text { education courses at a large } \\
\text { metropolitan university in Australia } \\
254 \text { responses received }\end{array}$ & $\begin{array}{l}17 \text { faculties with a total of } 1900 \\
\text { academic staff from various fields } \\
600 \text { surveys were sent randomly to } \\
\text { academic staff } \\
233 \text { responses received }\end{array}$ & $\begin{array}{l}936 \text { participants from the US Coast } \\
\text { Guard: } \\
\text { Sample 1: } 436 \text { respondents completed } \\
\text { initial ELRSA (40 items) } \\
\text { Sample 2: } 500 \text { respondents completed } \\
\text { revised ELRSA (27 items) } \\
\text { Sample 3: } 15 \text { respondents who have } \\
\text { experience in online training from } \\
\text { sample 2, completed revised ELRSA } \\
\text { and a seven-item survey of their self } \\
\text { perceived performance in e-learning }\end{array}$ \\
\hline Validation & & Cronbach's Alpha Statistic & $\begin{array}{l}\text { Cronbach's Alpha Statistic and } \\
\text { SPSS were used to perform factor } \\
\text { analysis }\end{array}$ & $\begin{array}{l}\text { Cronbach's Alpha Statistic and } \\
\text { SPSS } 10 \text { were used to perform } \\
\text { exploratory factors analysis }\end{array}$ & Cronbach's Alpha coefficient \\
\hline
\end{tabular}




\subsection{E-learning readiness instruments}

\subsubsection{E-Learning Readiness Survey}

Aydin and Tasci (2005) developed an E-Learning Readiness Survey (ELRS) to assess how managers perceive their organisation's readiness for e-learning in Turkey and to investigate whether managers' demographic characteristics (gender, age, education, and computer experience) differentiate their perception of organisational readiness for elearning. All respondents were directors of human resources departments or personnel/training departments. The instrument has four subscales: people; selfdevelopment; technology; and innovation. The researchers confirmed that the instrument has been validated for comprehension and is applicable to assess institutional readiness in other emerging countries. Cronbach's Alpha statistic was used to determine the reliability of ELRS. The overall score was quite high at 0.92 . The study revealed that although the companies surveyed were ready for e-learning overall, to successfully implement e-learning they needed to improve their human resources. The results confirmed that gender, age, education level, and computer experience had no effect on participants' overall perception of organisational readiness.

\subsubsection{E-Learning Readiness}

An E-Learning Readiness (ELR) instrument was developed by Abas, Kaur, and Harun (2004) to assess e-learning readiness in Malaysia. The study employed four instruments, one for each target group: policy makers, providers (private corporations, organisations, tertiary educational institutions and major technology providers), enablers (tutors, lecturers, and trainers), and receivers (learners and trainees). Each survey was divided into two sections: (1) demographic variables and (2) perceptions of readiness in eight areas: learner; management; content; personnel; technical; financial; environmental; and cultural. The study assessed policy makers' readiness to enable e-learning programmes within their respective organisations, providers' readiness to embark on elearning programmes, enablers' readiness to deliver e-learning programmes, and receivers' readiness for e-learning. The study revealed that enablers and receivers were less ready than policy makers and providers. The study confirmed that although there was a large amount of resources for management and technical facilities, more financial assistance was still needed to improve the infrastructure in Malaysia. Enablers and 
receivers also needed to improve their readiness in three areas: content, technical, and environmental. To ensure their environmental and cultural readiness, there must be thorough examination of procedures for resource allocation and technical initiatives.

Table 9. Overall means for e-learning readiness among groups of respondents

\begin{tabular}{|l|c|c|c|c|}
\hline \multicolumn{1}{|c|}{ Areas of Readiness } & Policy Maker & Provider & Enabler & Receiver \\
\hline 1. Learner & - & - & 5.73 & ${ }^{*} 6.33$ \\
\hline 2. Management & 5.98 & - & 6.24 & - \\
\hline 3. Personnel & 5.87 & 6.52 & 5.88 & - \\
\hline 4. Content & - & 6.24 & 5.91 & 5.88 \\
\hline 5. Technical & ${ }^{*} 6.14$ & ${ }^{*} 6.95$ & 5.95 & 5.59 \\
\hline 6. Environmental & ${ }^{\star \star} 4.76$ & ${ }^{\star \star} 4.77$ & ${ }^{\star} 5.27$ & ${ }^{\star \star} 5.39$ \\
\hline 7. Cultural & 6.02 & - & ${ }^{*} 6.77$ & 5.99 \\
\hline 8. Financial & 5.26 & 5.97 & 6.39 & 6.06 \\
\hline
\end{tabular}

(Abas, Kaur, \& Harun, 2004, p. 92)

* The highest mean $\quad{ }^{* *}$ The lowest mean

\subsubsection{Tertiary Students' Readiness for Online Learning}

An instrument to examine tertiary students' readiness for online learning (TSROL) was developed by Pillay, Irving, and Tones (2007). The instrument has four subscales: technical skills; computer self-efficacy; learner preferences; and attitude towards computers. Factor analysis was used to confirm the factors within a new sample. The results show an improvement of reliability in three subscales compared to a prior study by Pillay (2006). Three key points were identified: (1) the learner preferences subscale required revision as it had poor reliability and validity; (2) older students had lower technical skills and computer self-efficacy than younger students; and (3) TSROL can be improved by adopting a more multidimensional interpretation of the learning preferences and attitudes towards computers.

\subsubsection{Individual Readiness to Develop and Implement E-Learning}

Sadik (2007) developed an instrument to measure individual readiness to develop and implement e-learning (IRDI-EL). The target respondents were academic staff at South Valley University in Egypt. The study aimed to determine the state of readiness of academic staff at South Valley University in Egypt to implement e-learning strategies in their teaching; and how support systems and procedures for staff could be further developed, enabling the most effective and appropriate use of learning technologies and enhancing the student and staff experience. There were three subscales: (1) competencies; (2) experience; and (3) attitudes toward the development and 
implementation of e-learning in university teaching. The survey instrument was developed in four phases using both quantitative and qualitative methods: (1) delineation of relevant domains for the construct interest; (2) survey assembly and pilot testing; (3) large scale field testing; and (4) validation of instrument scores by using factor analysis and correlation methods. The study revealed that all three of the domains (competencies, experience and attitudes) affect faculty's individual readiness to successfully develop and implement e-learning approaches.

\subsubsection{E-Learning Readiness Self-Assessment}

The E-Learning Readiness Self-Assessment (ELRSA) was developed by Watkins, Leigh, and Triner (2004) to assess the readiness of individual learners who have no previous e-learning experience in an online learning environment. Cronbach's Alpha coefficient was used to determine the strength of the relationships among the items within each scale. Three different samples were used in developing the instrument from the initial self-assessment to the revised instrument. The revised instrument had six selfassessment categories: technology access; online skills and relationships; motivation; online audio/video; Internet discussions; and importance to your success. The researchers claimed that the six scales were reliable; however they only measure readiness from the perspective of learners. It could be valuable to assess perceived and actual success from multiple perspectives (instructors, supervisors, and learners). They also suggested future exploration of the distinction between technology skills and online relationships.

\subsection{The gaps}

Most of the e-learning readiness research has been conducted in developed countries. The instruments for assessing e-learning readiness were mainly formulated for organisations or institutions that were already familiar with OES. Moreover, most of those instruments were developed to assess only one particular type of respondent. Although the ELR (Abas, Kaur \& Harun, 2004) comprised four instruments to separately assess four types of respondent (policy makers, providers, enablers, and learners), the measurements were not comprehensive. Accordingly, it is cumbersome to assess the overall readiness of a population by comparing the levels of readiness in different respondents using four different measurements. 
Furthermore, this study identified 33 dimensions to measure e-learning readiness (Table 7) that had been found in previous research. It seems that some of these dimensions overlap one another. Thus, this study regroups these dimensions into a more concise set of dimensions to assess OES readiness. Additionally, it is important to close the gap by developing a comprehensive instrument that can be used to assess all respondents in a single set of surveys especially for a research context with no prior e-learning knowledge in a developing country context.

\subsection{Eight dimensions of OES readiness}

The 33 e-learning readiness dimensions in Table 7 were grouped into eight dimensions: (1) technological skills; (2) equipment/infrastructure; (3) online learning style; (4) attitude; (5) human resources; (6) cultural; (7) environmental; and (8) financial. These eight dimensions are used to measure the level of readiness for OES implementation in a research context with no prior e-learning knowledge specifically in a developing country context. The characteristics of the eight dimensions will be presented in the next section.

\subsubsection{Technological skills readiness}

Technological skills readiness refers to the observable and measurable technical competencies (Chapnick, 2000) involving users' capabilities using computers and the Internet (Aydin \& Tasci, 2005; Chan \& Ngai, 2007; Condie \& Livingston, 2007). Users with high levels of IT knowledge are more likely to adopt e-learning, whereas those who have a low level of IT knowledge tend to use traditional training methods such as lectures and workshops (Aydin \& Tasci, 2005; Chan \& Ngai, 2007).

\subsubsection{Online learning style readiness}

Users' online learning style readiness is critical to the success of OES implementation. Abas, Kaur, and Harun define learner readiness as "the readiness of the learner or trainee in terms of time commitment to E-learning, discipline and interest in E-learning as well as perception of the status of qualifications obtained via E-learning" (2004, p. 12). Although many private sector organisations are increasingly using the Internet to deliver training, there are still problems with the instructional reliability of e-learning 
and the readiness of learners to engage in an online learning environment (Watkins, Leigh, \& Triner, 2004). Learners are expected to continually adapt to the ever-changing online environment due to advances in technology. Holsapple and Lee-Post (2006) noted that the level of learners' readiness and openness towards the online learning environment can be assessed using several factors: academic preparedness; technical competence; lifestyle aptitudes; and learning preference towards e-learning.

Learners who are new to online learning tend to spend more time familiarising themselves with the online technology and trying to understand new approaches of online teaching, online learning, and online processes (Aydin \& Tasci, 2005; Welsh, et al., 2003). Aydin and Tasci (2005) noted that people in developing countries typically have stronger family bonds and tend to spend their spare time with family members rather than being involved in an online environment. They also noted that people there are more likely to work a fixed number of hours so they tend to spend most of the day in the workplace. Thus, time management skills are crucial for implementing successful OES.

\subsubsection{Equipment/infrastructure readiness}

Equipment and infrastructure are vital when adopting OES. Abas, Kaur, and Harun (2004) defined equipment/infrastructure readiness as the provision of technical support, e-learning content delivery, broadband facilities, and a Learning Management System (LMS) by the organisations who adopt the systems. Chapnick (2000) defined it as having the right equipment. The availability of hardware and software in an organisation should be identified within the assessment instrument (Aydin \& Tasci, 2005; Welsh, et al., 2003; Wild, Griggs, \& Downing, 2002). The questions should be developed to assess the level of IT infrastructure including the capabilities of hardware, software, and network within the organisation. The focus should be on accessible computers, accessible Internet/intranet, and the ease of use of the innovations.

\subsubsection{Attitude readiness}

User attitudes (Aydin \& Tasci, 2005; Chan \& Ngai, 2007; Condie \& Livingston, 2007), and user behaviour (Cook \& Dupras, 2004) are factors that influence the use of 
technology. Attitude readiness in this proposed study involves confidence, enjoyment, importance, motivation, self-development, and anxiety.

How to ensure learner motivation in an online environment is one of the attitude dimensions (Welsh, et al., 2003). Self-development relates to the self-motivation and enthusiasm attitude and behaviour toward the adoption of e-learning involving the eagerness to continuously learn and develop capabilities required for embracing the innovation. Organisations can adopt innovation earlier if they are open to organisational and individual development, actively seeking information about innovation to improve themselves, and have higher self-efficacy beliefs about achievement (Aydin \& Tasci, 2005). E-learning adoption will be easier if the organisations are willing to establish a budget for initiating organisational and individual development, have managers who believe in the power of self-development, and have employees who have positive attitudes towards developing themselves (Aydin \& Tasci, 2005).

\subsubsection{Human resources readiness}

Chapnick (2000) defines human resources readiness as the availability and design of the human support system. Therefore, human resources readiness involves management and personnel. Management readiness refers to the organisation having a vision/mission or formulated policies related to the provision of e-learning and the organisational recognition of qualifications obtained via e-learning (Abas, Kaur, \& Harun, 2004, p. 12). Personnel readiness refers to the readiness of the organisation in terms of having a central unit dedicated to e-learning initiatives with a team of dedicated instructional designers as well as a staff development plan for e-learning (Abas, Kaur, \& Harun, 2004, p. 13).

The people most likely to be involved in the assessment process are human performance technology (HPT) professionals (Haney, 2002). Condie and Livingston (2007) added that instructor, teacher, or trainer readiness is a vital element of successful e-learning implementation. Aydin and Tasci (2005) noted that the people factor involves all human resource characteristics of an organisation and organisations with more skilled staff are more likely to successfully adopt e-learning. They also claimed that users who have a higher level of education tend to adopt the innovation more than others. The education 
level of users may affect the adoption of innovation. Therefore, the education level of users can be used as one of the e-learning readiness predictors.

The appearance of an enthusiastic individual who has the knowledge, skills, responsibility, and authority to inspire and motivate the organisation is positively related to adoption of an innovation (Aydin \& Tasci, 2005; Chan \& Ngai, 2007). Another important predictor is the existence of a supportive individual from top level management who has sufficient IT knowledge and the authority to lead the organisation towards such an innovation (Aydin \& Tasci, 2005; Chan \& Ngai, 2007). E-learning providers (vendors) and/or consultants should also be taken into consideration as predictors of the adoption of e-learning.

\subsubsection{Environmental readiness}

Abas, Kaur, and Harun (2004, p. 12) defined environmental readiness as "a readiness of a society/nation for E-learning as perceived by the policy makers, providers, enablers and learners/trainees". This involves the readiness of the organisation as a whole in terms of government policy, the role of mass media, and intellectual property regulations. Chapnick (2000) defined it as the large scale forces operating on the stakeholders both within and outside the organisation.

\subsubsection{Cultural readiness}

Cultural readiness can be defined as "the enculturation of E-learning in terms of Internet use and networked technologies to disseminate information, communication, interaction and teaching" (Abas, Kaur, \& Harun, 2004, p. 12). Cultural readiness involves the readiness of all stakeholders (policy makers, enablers, and learners/trainees) to make e-learning a way of life, i.e. they accept and use it as a mode for teaching and learning in their organisation. Some organisations are able to easily adopt the innovation, while others may find it difficult. Aydin and Tasci (2005) noted that the past experiences of the users (employees and managers) with innovations in any or similar previous management procedures in an organisation may influence the results of an OES initiative. They suggested several questions to assess cultural readiness including acceptance of the systems among users, and potential barriers to implementation (internal or external, legal and/or political barriers) that may influence 
the applicability of e-learning in the organisation. Therefore, to measure organisational readiness for OES, acceptance or rejection of innovation in an organisation could be used as predictors.

\subsubsection{Financial readiness}

Abas, Kaur, and Harun (2004, p. 12) define financial readiness as "learner/trainee and institutional/organisational readiness to spend or allocate funds to develop and/or acquire E-learning". Chapnick (2000) noted that it generally refers to whether a learner/trainee or an institution/organisation is financially ready for e-learning programmes as perceived by policy makers, enablers, and learners/trainees. Financial readiness involves budget size and the funding allocation process.

The eight dimensions of OES readiness are put together in a single set of surveys, a comprehensive organisational e-learning readiness instrument (COERI) to measure all target respondents (policy makers, enablers, providers, and receivers). These eight dimensions were chosen particularly because they are relevant and well-suited to measure a research context with no prior e-learning knowledge. They are also the most common aspects that occur in most studies.

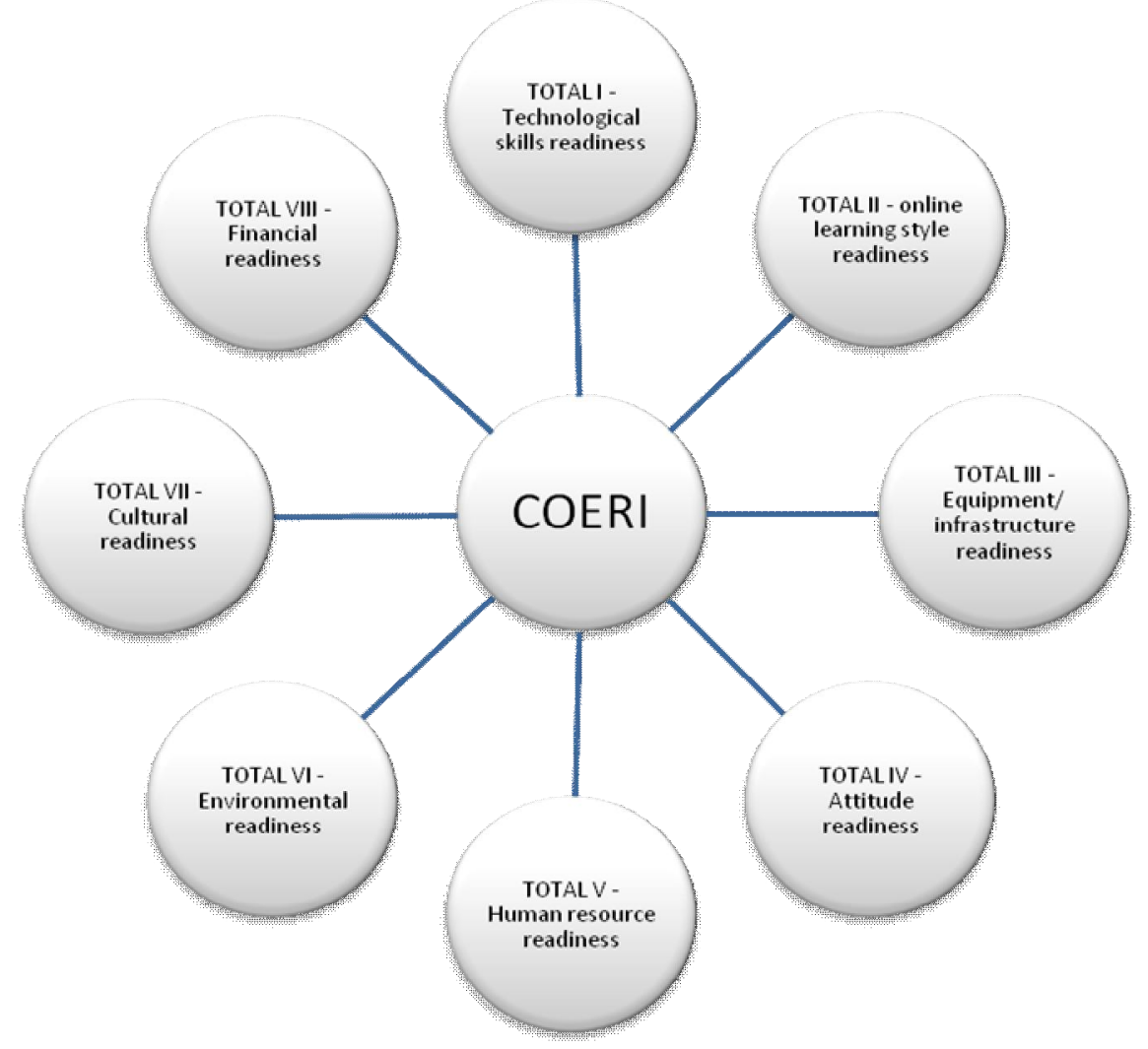

Figure 8: COERI - Eight dimensions 


\section{Chapter 3: Research Design and Methods}

This chapter presents the research design and methods used to perform the research for this study by explaining the methodological approach, research design, validity, reliability, and ethical issues. This chapter is organised as follows:

3.1 Research paradigm

3.2 Assumption and rationale

3.3 A mixed-methods approach

3.4 The role of the researcher

3.5 Ethical issues

3.6 Potential threats

3.7 Written report and presentation using a mixed-methods approach

3.8 Summary of research design and methodology

3.9 Research outline and timeframe

\subsection{Research paradigm}

The ontological approach of this research is pragmatism. The researcher believes that multiple paradigms can be used to address research problems (problem centric) (Creswell \& Plano-Clark, 2007). A mixed methods approach was used with a quantitative approach as the main data collection method complemented by a qualitative approach to enrich the results. Therefore, epistemologically, this study was based on the positivist tradition. Table 10 shows the list of assumptions that underline the study. 
Table 10. Quantitative, qualitative, and mixed methods paradigm assumptions

\begin{tabular}{|c|c|c|c|}
\hline Assumptions & Quantitative & Qualitative & Mixed Methods \\
\hline $\begin{array}{l}\text { Ontological } \\
\text { What is the nature of } \\
\text { reality? }\end{array}$ & $\begin{array}{l}\text { Reality is objective and singular, } \\
\text { apart from the researcher } \\
\text { (singular reality) } \\
\text { Postpositivism }\end{array}$ & $\begin{array}{l}\text { Reality is subjective and multiple as } \\
\text { seen by participants in a study } \\
\text { (multiple realities) } \\
\text { Constructivism }\end{array}$ & $\begin{array}{l}\text { Reality is both subjective and } \\
\text { objective (singular and } \\
\text { multiple realities) } \\
\text { Pragmatism }\end{array}$ \\
\hline $\begin{array}{l}\text { Epistemological } \\
\text { What is the } \\
\text { relationship between } \\
\text { the researcher and } \\
\text { what is being } \\
\text { researched? }\end{array}$ & $\begin{array}{l}\text { The researcher is independent from } \\
\text { what is being investigated } \\
\text { Distance and impartiality (the } \\
\text { researcher objectively collects } \\
\text { data on instruments) }\end{array}$ & $\begin{array}{l}\text { The researcher interacts with what is } \\
\text { being investigated } \\
\text { Closeness (the researcher visits } \\
\text { participants at their sites to collect } \\
\text { data) }\end{array}$ & $\begin{array}{l}\text { The researcher is independent } \\
\text { from the phenomenon being } \\
\text { studied; and also interacts } \\
\text { with what is being researched } \\
\text { Practically (the researcher } \\
\text { collects data by using "what } \\
\text { works" to address research } \\
\text { questions) }\end{array}$ \\
\hline $\begin{array}{l}\text { Axiological } \\
\text { What is the role of } \\
\text { values? }\end{array}$ & $\begin{array}{l}\text { Value-free and unbiased (the } \\
\text { researcher uses checks to } \\
\text { eliminate bias) }\end{array}$ & $\begin{array}{l}\text { Value-laden and biased (the } \\
\text { researcher actively talks about } \\
\text { their biases and interpretations) }\end{array}$ & $\begin{array}{l}\text { Multiple stances (the researcher } \\
\text { includes both biased and } \\
\text { unbiased perspectives) }\end{array}$ \\
\hline $\begin{array}{l}\text { Methodological } \\
\text { What is the process } \\
\text { of research? }\end{array}$ & $\begin{array}{l}\text { Deductive process } \\
\text { Cause and effect } \\
\text { Static design - categories isolated } \\
\quad \text { before study } \\
\text { Context-free } \\
\text { Generalisation leading to prediction, } \\
\quad \text { explanation, and understanding } \\
\text { Accurate and reliable through } \\
\quad \text { validity and reliability }\end{array}$ & $\begin{array}{l}\text { Inductive process } \\
\text { Mutual simultaneous shaping of factors } \\
\text { Emerging design - categories identified } \\
\quad \text { during research process } \\
\text { Context-bound } \\
\text { Patterns and theories developed for } \\
\text { understanding } \\
\text { Accurate and reliable through } \\
\quad \text { verification }\end{array}$ & $\begin{array}{l}\text { Combining deductive and } \\
\text { inductive (the researcher } \\
\text { collects both quantitative and } \\
\text { qualitative data and mixes } \\
\text { them }\end{array}$ \\
\hline $\begin{array}{l}\text { Rhetorical } \\
\text { What is the } \\
\text { language of the } \\
\text { research? }\end{array}$ & $\begin{array}{l}\text { Formal } \\
\text { Based on set definitions } \\
\text { Impersonal voice } \\
\text { Use of accepted quantitative words }\end{array}$ & $\begin{array}{l}\text { Informal } \\
\text { Evolving decisions } \\
\text { Personal voice } \\
\text { Use of accepted qualitative words }\end{array}$ & $\begin{array}{l}\text { Formal or informal (the researcher } \\
\text { may employ both formal and } \\
\text { informal styles of writing) }\end{array}$ \\
\hline
\end{tabular}

Modified from Creswell, 1994, p. 5; Creswell \& Plano-Clark, 2007, p. 24

\subsection{Assumptions and rationale}

A mixed methods approach involves philosophical assumptions that guide the direction of data collection and analysis and the mixture of qualitative and quantitative approaches throughout the research process (Creswell \& Plano-Clark, 2007). The approach focuses on collecting, analysing, and mixing both quantitative and qualitative data in either a single study or series of studies. The primary characteristic is that it uses a combination of quantitative and qualitative approaches to provide a better understanding of research problems than either approach alone (Creswell \& PlanoClark, 2007). All data were collected and analysed separately and independently using each traditional techniques associated with each data type. Afterwards they were integrated within the interpretation.

The mixed methods approach was chosen to overcome weaknesses of each method if they are implemented alone. According to Creswell and Plano-Clark (2007) quantitative methods' weaknesses include: inadequacy for understanding the context or setting in which people talk; not giving the voices of respondents; and the position of the researchers who are in the background and not part of the research so their personal biases and interpretations are seldom discussed. On the other hand, qualitative studies 
are believed to be unscientific, exploratory, entirely personal, and the interpretations made by the researchers are full of biases (Creswell, 1994; Denzin \& Lincoln, 1994). Qualitative methods also focus on studying human experiences, and are not aimed at generalising the study results or generalising the findings to a larger group than the number of participants (Creswell \& Plano-Clark, 2007).

The study aims to generalise from a sample to a population in identifying the level of organisational readiness for OES particularly in a developing country. The level of FORDA's readiness for OES has not been addressed. Little research has been done into assessing OES readiness in a developing country context. Therefore, a mixed methods approach was used to capture the best of both qualitative and quantitative data as the researcher wanted to both generalise the findings to a population and develop a detailed view of the meaning of a phenomenon or concept for individuals.

The researcher surveyed a large number of individuals within the research context, and interviewed twelve policy makers at FORDA to obtain their specific expressions and views about the topic. The survey design was used as it provides a quantitative or numeric description of trends, attitudes, or opinions of a population by investigating a sample of that population (Creswell, 2003 p. 153). Therefore, from the sample results the researcher can generalise or make claims about the population.

The researcher was familiar with the qualitative approach and decided to use mixed methods in order to conduct a more comprehensive study. Finally, the approach was selected to accommodate diverse audiences of this study including colleagues in the field of study, policy makers at FORDA and practitioners. These audiences may have diverse experience including qualitative, quantitative, and mixed methods studies.

\subsection{A mixed-methods approach}

The data collection was conducted in two parts by using quantitative and qualitative methods separately. The main data collection was a cross sectional survey complemented by twelve semi-structured interviews. Both quantitative results and qualitative findings were integrated into the interpretation part. Participation in either part was voluntary for all participants. The Secretary of FORDA provided letters of support which were addressed to all forestry directors in 20 FORDA offices across the 
regions. The letters were attached to the survey instrument, information sheet, and participation consent form for the interview. The researcher had an opportunity to meet all the forestry directors in their annual meeting in early August 2008 to conduct interviews. The survey kits consisted of the survey in both hardcopy and softcopy (CD), and the information sheet (Appendix 10) including the link to the online survey were handed over to each branch office at that time. The survey was available to participants in both paper and online form to maximise the number of participants. Surveys in hardcopy were also sent to each office. The online survey link was also sent via email to key people in each organisation to be disseminated. Follow up notices were sent to each organisation twice to collect the survey.

\subsubsection{Phase I: Confirmatory - Survey}

The online survey was created by using SurveyMonkey.com, an online survey tool. The online survey link and softcopy survey were delivered via email for efficiency. This was also done to observe how many respondents preferred to participate through online means. The paper-based survey was also sent by conventional post to reach respondents who are not familiar with online means or those who cannot be reached by online channels. The email invitations were sent to the same group that received paper surveys. Due to the dispersed locations, and in order to minimise expenses and save time, the emails were sent to several key people including researchers, research assistants, managers, and/or general employees at each branch office. They were asked to forward the online survey link via email or to print the soft copy, then distribute the surveys to their colleagues at their office. Therefore, although the number of specific types of respondents (managers, researchers, research assistants, IT personnel, and general employees) had been previously determined, once the surveys were sent the researcher had no control over which respondents would participate in the research.

\subsubsection{Quantitative data collection: Population and sample}

The target population was employees of FORDA. The size of the population was 1,640. The sample frame was a list of employees within 20 FORDA branch offices across all regions. The study used a stratified sampling design in which the research context was divided into four strata based on the job title: researchers, research assistants, managers, and general employees. 
The reporting unit consisted of individual FORDA employees, including managers (forestry directors, mid-level and low-level managers), researchers, research assistants (including IT personnel), and general employees. The level of observation was each FORDA branch office and employee. Table 11 presents the number in each stratum of each branch office.

Table 11. Sampling frame: Profiles of FORDA

\begin{tabular}{|c|c|c|c|c|c|c|}
\hline \multirow{2}{*}{ No. } & \multirow{2}{*}{ FORDA regional Offices } & \multicolumn{4}{|c|}{ Human Resources } & \multirow{2}{*}{$\begin{array}{l}\text { Tota } \\
\text { Size }\end{array}$} \\
\hline & & $\mathbf{R}$ & RA & M & GE & \\
\hline 1. & Secretariat of FORDA, Jakarta & - & - & 18 & 83 & 101 \\
\hline 2. & Centre for Forest and Nature Conservation Research \& Development (CFNCRD), BOGOR & 57 & 35 & 10 & 77 & 179 \\
\hline 3. & Centre for Forest Products Research \& Development (CFPRD), BOGOR & 51 & 33 & 10 & 61 & 155 \\
\hline 4. & Centre for Socio-Economic and Forestry Policy Research (CSEFPR), BOGOR & 28 & 1 & 9 & 25 & 63 \\
\hline 5. & Centre for Plantation Forest Research and Development (CPFRD), BOGOR & 18 & 9 & 10 & 18 & 55 \\
\hline 6. & Centre for Dipterocarp Research (CDR), SAMARINDA & 26 & 19 & 10 & 39 & 94 \\
\hline 7. & Centre for Biotechnology and Forest Tree Improvement Research (CBFTIR), YOGYAKARTA & 36 & 27 & 9 & 48 & 120 \\
\hline 8. & Forestry Research Institute (FRI) of AEK NAULI & 21 & 23 & 5 & 42 & 91 \\
\hline 9. & FRI of MAKASSAR & 27 & 23 & 5 & 52 & 107 \\
\hline 10. & FRI of KUPANG & 19 & 12 & 4 & 37 & 72 \\
\hline 11. & FRI of MANOKWARI & 22 & 24 & 3 & 18 & 67 \\
\hline 12. & FRI of PALEMBANG & 27 & 12 & 5 & 42 & 86 \\
\hline 13. & FRI of BANJARBARU & 14 & 19 & 5 & 33 & 71 \\
\hline 14. & FRI of MATARAM & 14 & 12 & 4 & 5 & 35 \\
\hline 15. & FRI of MANADO & 10 & 12 & 5 & 5 & 32 \\
\hline 16. & FRI of SURAKARTA & 25 & 20 & 5 & 34 & 84 \\
\hline 17. & FRI of CIAMIS & 17 & 14 & 4 & 26 & 61 \\
\hline 18. & Forest Breeding Technology Research Institute (FBTRI) of BOGOR & 21 & 20 & 4 & 32 & 77 \\
\hline 19. & FBTRI of SAMBOJA & 15 & 16 & 4 & 12 & 47 \\
\hline 20. & Pulp and Fiber Forest Products Research Institute (PFFPRI) of KUOK & 16 & 17 & 4 & 6 & 43 \\
\hline & TOTAL SIZE & 464 & 348 & 133 & 695 & 1640 \\
\hline
\end{tabular}

R = Researchers; RA = Research assistants; $\mathbf{M}$ = Managers ; GE = General employees

The sample size was determined by using Yamane's formula (1967) with a 95\% confidence level which produces approximately 755 samples (Bartlett, Kotrlik, \& Higgins, 2001; Israel, 2003). Table 12 shows the sample size estimation. Afterwards, as shown in Table 13, Cochran's formula was used to determine the minimum returned sample size for the given population size (Bartlett, Kotrlik, \& Higgins, 2001; Israel, 2003).

Table 12. Sample size estimation

\begin{tabular}{|c|c|c|c|c|}
\hline \multirow[t]{2}{*}{ Stratum } & \multirow[t]{2}{*}{$\begin{array}{l}\text { Population } \\
\text { Size } \\
\text { (N) }\end{array}$} & \multirow[t]{2}{*}{$\begin{array}{l}\% \text { each stratum } \\
\text { (A) }\end{array}$} & \multicolumn{2}{|c|}{$\begin{array}{l}\text { Sample size (n) for Precision (e) } \\
\text { using Equation (5) from Israel (2003) } \\
n=N / 1+N(e)^{2} \\
\text { (e) }\end{array}$} \\
\hline & & & $5 \%$ & $3 \%$ \\
\hline Researchers & 464 & 28.292683 & 215 & 327 \\
\hline Research assistants & 348 & 21.219512 & 186 & 265 \\
\hline Managers & 133 & 8.1097561 & 100 & 119 \\
\hline General employees & 695 & 42.378049 & 254 & 428 \\
\hline Total & 1640 & & 755 & 1139 \\
\hline
\end{tabular}


Table 13. Minimum returned sample size for a given population size for continuous and categorical data

\begin{tabular}{|c|c|c|c|c|c|c|}
\hline \multirow{3}{*}{ Size of population (N) } & \multicolumn{6}{|c|}{ Size of sample (n) } \\
\hline & \multicolumn{3}{|c|}{$\begin{array}{l}\text { Continuous data } \\
\text { acceptable error } 3 \% \\
\text { alpha }=.05 ; \mathrm{t}=1.96\end{array}$} & \multicolumn{3}{|c|}{$\begin{array}{l}\text { Categorical data } \\
\text { acceptable error } 5 \% \\
p=0.25 ; t=1.96\end{array}$} \\
\hline & no & $\mathrm{n} 1$ & n2 & no & $\mathrm{n} 1$ & n2 \\
\hline 1640 & 267 & 229 & 353 & 288 & 245 & 377 \\
\hline
\end{tabular}

\subsubsection{The instrument}

To measure organisational readiness for OES, the study proposes eight dimensions of readiness drawn from the literature review: (1) technological skills; (2) online learning style; (3) equipment/infrastructure; (4) attitude; (5) human resource; (6) environmental; (7) cultural; and (8) financial. The instrument is attached in Appendix 12. The questionnaire was divided into three sections: A, B, and C. Section A: Demographic contains nine questions to collect demographic characteristics from the individuals including age, gender, education level, the organisation they belong to, and their position in the organisation. Section B: Prior e-learning knowledge - contains nine questions to collect individuals' prior knowledge about e-learning and their computer literacy. Section C: E-learning readiness dimensions - contains eight variables of elearning readiness dimensions. A five-point Likert scale was used to ask about opinions.

\subsubsection{Quantitative data analysis}

The data was analysed by using the Statistical Package for Social Sciences (SPSS) version 16.0. The number of responses sent and returned is reported in Chapter 4. Factor analyses were performed to evaluate and test the eight scales. Biases were identified, addressed, and reported. Afterwards, descriptive and inferential analyses of the data for all independent and dependent variables in the study were presented including the means, standard deviations, and range of scores for these variables (Appendix 5).

\subsubsection{Quantitative validation}

Data validation was conducted to check the data quality and the research results. Creswell and Plano-Clark (2007) noted that quantitative research typically uses validity to draw meaningful inferences from the results to a population; and reliability to check the consistency and stability of the scores received from participants over time. They also noted that the standards of quantitative validity can be drawn from a source external to the researcher and the participants, statistical procedures or external experts. The survey had been pilot tested by sending the initial draft to the research supervisor and several peer reviewers. Subsequently, in order to gain validity and reliability from the research context, a field test had also been conducted by sending the initial survey to 
a number of colleagues at FORDA. The reliability of the scales was analysed by using Cronbach's Alpha statistic.

\subsubsection{Phase II: Exploratory - Semi-Structured Interviews}

The research was a triangulation study using multiple data sources in a single study in which data from surveys were compared with data obtained from individuals' interviews for consistency. The research employed a single case research strategy in which the researcher investigated a case with clear boundaries in the FORDA context (Creswell, 1998; Yin, 2003). This design was chosen to explore the nature of organisational readiness for OES from the viewpoint of policy makers at FORDA.

\subsubsection{Qualitative data collection}

Denzin and Lincoln (1994) and Creswell (1998) noted that qualitative data may consist of various empirical materials describing habitual and problematic moments and meaning in the lives of individuals including: case studies, personal experiences, introspection, life stories, interviews, and observational, historical, interactional, and visual texts. The qualitative data were collected through 12 semi-structured interviews to complement the quantitative results. The respondents were policy makers at FORDA. Interviewees could also participate in the survey. One-on-one interviews were conducted face-to-face for approximately one hour and were recorded by using a recording device. The data collection was based on open-ended questions. The researcher also observed the natural setting and used available documents that were relevant to the research purpose. However, only one other office was visited to interview a policy maker. The researcher has contextual material available and a wide array of information about the case to provide an in-depth picture and setting for the case as the researcher is an employee of FORDA.

\subsubsection{Qualitative data analysis}

Creswell (2003) noted that qualitative data analysis involves making a detailed description of the case and its setting, making an interpretation, drawing conclusions about its meaning personally and theoretically, stating the lessons learned, and offering further questions to be asked. The data was analysed and interpreted in an ongoing process throughout the research process involving continual reflection about the data including asking analytical questions, writing memos, mind mapping, categorising, 
discerning themes, examining their relationships, and putting them into a matrix in order to generate a plausible conclusion (Creswell, 2003; Yin, 1994).

\subsubsection{Qualitative validation}

Creswell and Plano-Clark (2007) noted that qualitative validation is focused on assessing whether the information gathered from the data is accurate. Creswell (2003) explained that qualitative research outweighs validity by determining whether the findings are accurate from the perspective of the researchers, the participants, or the readers of an account. It mainly deals with trustworthiness, authenticity, and credibility in verifying the research results. Member checking was used in this study by sending back the interview transcripts to each participant for confirmation. Potential biases were presented in relation to the researcher's personal judgment. Any negative or discrepant information captured in the process was revealed. Peer reviewers and external auditors were employed to assess the research project.

\subsection{The role of the researcher}

The researcher is bound to the research context. The researcher is an employee of FORDA who is currently on study leave, and her previous main duty was organising communication and dissemination in the Forestry Research Institute of Solo. Her main responsibility was to administer publication and dissemination of the research and development results. Due to researcher introspection, it is likely that biases, values, and personal interests (reflexivity) in how the study was shaped might occur (Creswell, 2003). It is highly likely that the researcher recognised or had a connection with some of the participants. Therefore, data collection might be convenient and easy, but the issues of reporting data that are biased, incomplete, or compromised are still relevant.

\subsection{Ethical issues}

A formal letter and brief research proposal were sent to the Secretary of FORDA to request approval and support to conduct research in the FORDA setting involving a survey and semi-structured interviews. The researcher is obliged to respect the rights, needs, values, and desires of the research participants in both qualitative and quantitative methods of data collection. Therefore the Human Ethics Committee (HEC) application was submitted to Victoria University's Human Ethics Committee in order to 
evaluate the ethical considerations of the research (Appendix 9). All data collected from both quantitative and qualitative methods will remain strictly private and confidential. The data collection was carried out with the understanding of ensuring the confidentiality and security of materials. Sensitive materials obtained from the interviews were considered confidential - i.e., no one except the principal researcher and the research supervisor, both at Victoria University, is permitted to see it. The research participants were not identified in the final report. The materials (interview tapes, notes, and questionnaire results) will be kept secured and will be destroyed within two years of the completion of the research project. No copies will be made. Interview participants were able to withdraw from the research project without explanation, and any data provided would be returned to them or destroyed. The results of this study may be published and disseminated in a conference report at academic or professional conferences or through publication in academic or professional journals.

\subsection{Potential threats}

The mixed-methods approach is complicated, takes time, and requires a considerable amount of resources in collecting, analysing, and interpreting both quantitative and qualitative data. Possible threats may arise during the data collection and analysis processes. Creswell and Plano-Clark (2007) noted that a mixed-methods approach is prone to issues such as if the results from both quantitative and qualitative results do not agree, leading to the requirement for additional data collection. Table 14 shows potential threats to a mixed methods approach and how to minimise them.

Table 14. Potential threats and how to address them

\begin{tabular}{|c|c|c|}
\hline Processes & Threats & Minimising Threat \\
\hline \multirow[t]{4}{*}{$\begin{array}{l}\text { Data } \\
\text { collection }\end{array}$} & $\begin{array}{l}\text { Selecting different individuals for the qualitative and } \\
\text { quantitative data collection }\end{array}$ & Draw quantitative and qualitative samples from same population \\
\hline & $\begin{array}{l}\text { Unequal sample sizes: a small number of qualitative } \\
\text { and a large number of quantitative data }\end{array}$ & Use large qualitative samples or weight the cases \\
\hline & Not following up on contradictory results & Follow up on contradictory results or re-examine data \\
\hline & Introducing potential bias through data collection & Use unobtrusive data collection procedures \\
\hline \multirow[t]{4}{*}{ Data analysis } & Inadequate data transformation approaches & $\begin{array}{l}\text { Keep the transformation straightforward (e.g. count codes or } \\
\text { themes and weight them) }\end{array}$ \\
\hline & $\begin{array}{l}\text { Inadequate approaches to converging the data (e.g. } \\
\text { uninterruptible matrix) }\end{array}$ & $\begin{array}{l}\text { Develop a matrix with quantitative categorical data and } \\
\text { qualitative themes }\end{array}$ \\
\hline & Not addressing validity issues & Address potential issues for quantitative and qualitative validity \\
\hline & Two types of data do not address the same question & $\begin{array}{l}\text { Address the same question in both quantitative and qualitative } \\
\text { approaches }\end{array}$ \\
\hline
\end{tabular}

Modified from Creswell \& Plano-Clark, 2007, p. 147 


\subsection{Written report and presentation using a mixed-methods approach}

Creswell and Plano-Clark (2007) affirmed that presenting quantitative analysis involves summarising statistical results that depict trends and distributions of the data in a visual form including figures, bar charts, scatter plots, line graphs, or charts. Presenting qualitative results entails a discussion of the evidence for the themes or categories, and the presentation of figures that illustrate the physical setting of the study or frameworks, models, or theories. The structure of this written report was made as concise as possible. Clarity is particularly important in presenting the results of mixed-methods research. Therefore, the explanation of research processes and results has been divided into several separate sections. Visual images are used to simplify and summarise the ideas including tables, figures, and graphs. The researcher combined the writing styles of both quantitative and qualitative methods.

\subsection{Summary of research design and methodology}

Table 15 presents the summary of the data collection procedures of the mixed-methods approach. Subsequently, Table 16 provides the summary of qualitative and quantitative data analysis procedures.

\subsection{Research outline and timeframe}

The research was conducted approximately within fourteen months from March 2008 to April 2009. Table 17 provides an outline and the time frame of the research.

Table 15. Qualitative and quantitative data collection procedures

\begin{tabular}{|l|l|l|}
\hline $\begin{array}{l}\text { Phases in the Process of } \\
\text { Research }\end{array}$ & \multicolumn{1}{|c|}{ Qualitative } & \multicolumn{1}{c|}{ Quata Collection } \\
\cline { 2 - 3 } & $\begin{array}{l}\text { Qurposeful sampling strategies: selecting } \\
\text { participants who have experience with the central } \\
\text { phenomenon being studied } \\
\text { Small number of participants and sites: providing in- } \\
\text { depth information about each person or site }\end{array}$ & $\begin{array}{l}\text { Random sampling: Individuals are randomly selected } \\
\text { so that the resulting sample on average are } \\
\text { representative of a population (each individual in the } \\
\text { population has an equal chance of being selected) } \\
\text { Adequate size to reduce sampling error and provide } \\
\text { sufficient power }\end{array}$ \\
\hline Permissions needed & $\begin{array}{l}\text { From individuals providing access to sites; } \\
\text { Institutional review boards; Individuals }\end{array}$ & $\begin{array}{l}\text { From individuals providing access to sites; Institutional } \\
\text { review boards; Individuals }\end{array}$ \\
\hline Information to be collected & $\begin{array}{l}\text { Open-ended interviews; Open-ended observations; } \\
\text { Documents; Audiovisual materials }\end{array}$ & Survey \\
\hline Recording the data & Interview protocols; Observational protocols & Instruments with scores that are reliable and valid \\
\hline $\begin{array}{l}\text { Administering data } \\
\text { collection }\end{array}$ & $\begin{array}{l}\text { Attending to field issues } \\
\text { Attending to ethical issues }\end{array}$ & $\begin{array}{l}\text { Standardisation of procedures } \\
\text { Attending to ethical issues }\end{array}$ \\
\hline
\end{tabular}

Modified from Creswell \& Plano-Clark, 2007, p. 111 
Table 16. Qualitative and quantitative data analysis procedures

\begin{tabular}{|c|c|c|}
\hline \multirow{2}{*}{$\begin{array}{l}\text { General Procedures in } \\
\text { Data Analysis }\end{array}$} & \multicolumn{2}{|c|}{ Data Analysis Procedures } \\
\hline & Qualitative & Quantitative \\
\hline $\begin{array}{l}\text { Preparing the data for } \\
\text { analysis }\end{array}$ & $\begin{array}{l}\text { Organising documents and visual data } \\
\text { Transcribing text } \\
\text { Preparing the data for computer analysis }\end{array}$ & $\begin{array}{l}\text { Coding data by assigning numeric values } \\
\text { Cleaning the database } \\
\text { Recoding or computing new variables for computer } \\
\text { analysis } \\
\text { Establishing a codebook }\end{array}$ \\
\hline Exploring the data & $\begin{array}{l}\text { Reading through the data } \\
\text { Writing memos } \\
\text { Developing a qualitative codebook }\end{array}$ & $\begin{array}{l}\text { Visually inspecting data } \\
\text { Conducting a descriptive analysis } \\
\text { Checking for trends and distributions }\end{array}$ \\
\hline Analysing the data & $\begin{array}{l}\text { Coding the data } \\
\text { Assigning labels to codes } \\
\text { Grouping codes into themes (or categories) } \\
\text { Interrelating themes (or categories) or abstracting to } \\
\text { a smaller set of themes }\end{array}$ & $\begin{array}{l}\text { Choosing an appropriate statistical test } \\
\text { Analysing the data to answer research questions } \\
\text { Reporting inferential tests, effect sizes, and confidence } \\
\text { intervals } \\
\text { Using quantitative statistical software programs }\end{array}$ \\
\hline $\begin{array}{l}\text { Representing the data } \\
\text { analysis }\end{array}$ & $\begin{array}{l}\text { Representing findings in discussions of themes or } \\
\text { categories } \\
\text { Presenting visual models, figures, and tables }\end{array}$ & $\begin{array}{l}\text { Representing results in statements of results } \\
\text { Providing results in tables and figures }\end{array}$ \\
\hline Validating the data & $\begin{array}{l}\text { Using researcher, participant, and reviewer } \\
\text { standards } \\
\text { Employing validation strategies (member-checking, } \\
\text { peer review) }\end{array}$ & $\begin{array}{l}\text { Using external standards } \\
\text { Validating and checking the reliability of scores from past } \\
\text { instrument use } \\
\text { Establishing validity and reliability of current data }\end{array}$ \\
\hline $\begin{array}{l}\text { Specific mixed-methods } \\
\text { analysis techniques }\end{array}$ & \multicolumn{2}{|c|}{$\begin{array}{l}\text { Keeping the analysis of both quantitative and qualitative data separate during the research process. } \\
\text { Merging the two datasets in the interpretation phase in order to answer the mixed-methods questions. } \\
\text { Considering specific questions in order to answer the research questions including: to what extent do the } \\
\text { quantitative and qualitative data converge? How and why? To what extent do the same types of data confirm each } \\
\text { other? To what extent do open-ended themes support the survey results? What similarities and differences exist } \\
\text { across levels of analysis? }\end{array}$} \\
\hline $\begin{array}{l}\text { Validating the data in } \\
\text { mixed-methods } \\
\text { research }\end{array}$ & \multicolumn{2}{|c|}{$\begin{array}{l}\text { Drawing meaningful and accurate conclusions from all of the data in the study } \\
\text { Reinforcing "inference quality" or the accuracy with which the researcher draws inductive and deductive } \\
\text { conclusions from a study } \\
\text { Triangulation validity can be achieved by drawing evidence from different datasets (quantitative and qualitative) } \\
\text { that provide better results than either dataset alone }\end{array}$} \\
\hline
\end{tabular}

Modified and summarised from Creswell \& Plano-Clark, 2007, p. 129

Table 17. Research outline and timeframe (March 2008 - April 2009)

\begin{tabular}{|l|c|}
\hline \multicolumn{1}{|c|}{ Activities } & Timeframe \\
\hline Literature and library database search & March 2008 \\
\hline Initial annotated bibliography & March 2008 \\
\hline Research method selection & March 2008 \\
\hline Applying NZAID support for HLR & March 2008 \\
\hline Brief research proposal & April 2008 \\
\hline Literature review (database search) & April 2008 \\
\hline Developing in-depth methodology & April - May 2008 \\
\hline Interim research proposal & May - July 2008 \\
\hline Peer review, editing, and submitting final research proposal & December 2008 \\
\hline Re-submitting final research proposal & May - July 2008 \\
\hline Draft questionnaire/survey, final draft questionnaire & June - July 2008 \\
\hline Pilot testing at PHD group and FORDA & June - August 2008 \\
\hline Applying to HEC & July - November 2008 \\
\hline Contacting research participants & August 2008 \\
\hline Interviewing key people & August - November 2008 \\
\hline Sending online surveys & August - November 2008 \\
\hline Sending paper-based surveys & August - December 2008 \\
\hline Collecting survey results, transcribing interviews & December 2008 - February 2009 \\
\hline Statistical consultation & November - March 2009 \\
\hline Analysing quantitative data and writing up findings & February - March 2009 \\
\hline Analysing qualitative data and writing up findings & February - April 2009 \\
\hline Interim final written report & February - April 2009 \\
\hline Reviewing, editing, validation, and verification & February - April 2009 \\
\hline Writing up final written report & February - April 2009 \\
\hline Reviewing and editing & April 2009 \\
\hline Photocopying and binding & \\
\hline Submitting hard copies & - \\
\hline
\end{tabular}




\section{Chapter 4: Phase I - Confirmatory (Survey)}

This chapter describes the first phase of the research. It explains data collection and analysis procedures, findings, and discussion of the quantitative data. The software package SPSS version 16.0 was used to analyse the quantitative data. This chapter presents a detailed explanation of the analysis which was conducted in three main steps:

\section{Step ONE: Assessing internal consistency}

This stage involved the assessment of reliability and factor analysis of the eight constructs/dimensions (scale TOTAL I - TOTAL VIII). Factor analysis was performed to ensure that the instrument was sufficiently valid and reliable for the subsequent analysis.

\section{Step TWO: Performing multiple regression analysis and construct evaluation}

This stage entailed multiple regression analysis to determine significant predictors of dimensions of COERI. It also involved the evaluation of the twelve constructs of COERI.

Step THREE: Evaluating each readiness dimension and addressing the first and second research questions

This stage involved the evaluation of each readiness dimension. It also addressed the first research question: What is the level of organisational readiness to implement OES at FORDA?, and the second research question: What are the critical factors that need to be considered in order to implement successful OES at FORDA?

This chapter is organised as follows:

4.1 The survey

4.2 Demographics

4.3 Step One: Assessing internal consistency

4.4 Step Two: Multiple regression analysis and construct evaluation

4.5 Step Three: Dimension readiness evaluation

4.6 Summary 


\subsection{The survey}

The data was carefully gathered before conducting data analysis. The development of COERI has been discussed thoroughly in Chapter 2 and Chapter 3. The questions within the survey were adapted from five studies (Table 8, page 34). They were modified and customised specifically to the research context. The determination of sample for this research has been discussed thoroughly in Chapter 3.

The survey was pilot tested before being used for data collection. Before conducting pilot testing, the COERI was reviewed by Victoria University's Human Ethics Committee. The questionnaire was revised according to their comments. Subsequently, the first pilot tests were performed using this revised online survey (English version) in the $\mathrm{PhD}$ group of the School of Information Management at Victoria University of Wellington and in FORDA. The online survey was sent to a number of policy makers and colleagues at FORDA via email and through ICT Dephut, an online information and communication technology group administered by the Ministry of Forestry of Indonesia.

After these pilot tests, the COERI was again revised according to the feedback received. Afterwards, the revised COERI was translated from English into Indonesian. Several reviewers from FORDA were involved in the translation process to make sure that they understood the context of the Indonesian version, and to assure that both versions were as precise as possible without becoming too difficult to understand.

Next, the second pilot test of the Indonesian version of the questionnaire was performed in FORDA. This version was again revised. In order to maintain accuracy, any revision that occurred within the Indonesian version led to the same revision of the English version. The English version was also revised and adjusted according to the feedback received from this pilot test.

Once the data were gathered, duplicate responses were removed. Several respondents submitted both online and paper-based surveys. It was decided to use the online submissions for data analysis, so the paper duplicates were removed. 
The data were gathered by sending email invitations (online surveys) and by sending paper-based surveys by post. The online surveys were sent to the same group that received paper-based surveys. A total of 20 survey kits were sent to 20 regional FORDA offices. Each survey kit contained a set of paper-based surveys, a soft copy of the survey (on a CD) a covering letter and an information sheet with the online survey link on it. The content of the paper-based and online surveys was identical. The survey kits were delivered to 20 forestry directors or their subordinates who attended the FORDA annual meeting in Bali. Afterwards, a set of paper-based surveys were delivered to 20 regional offices by post.

\subsection{Demographics}

This section presents information on the respondents who participated in the study. The number of paper-based surveys sent varied across branch offices depending on the number of samples needed in each group within each branch office. In total, 672 paperbased survey forms were sent to 20 offices. Of those, 422 responses $(62.80 \%)$ were received whether online or paper-based. However, only 288 responses $(42.86 \%)$ were valid and used for data analysis. Of the 422 responses returned, 376 respondents (89.10\%) submitted paper-based survey forms, whilst only 46 respondents $(10.90 \%)$ submitted online survey forms (Figures 9 and 10). A more detailed descriptive analysis is presented in Appendix 1. Table 18 summarises the total number of surveys sent and returned, as well as the calculation of the return rate in each stratum. Figure 9 depicts the total number of surveys sent and returned in each stratum. Figure 10 illustrates the survey return rate in each stratum.

Table 18. Total number of survey sent/returned and return rate

\begin{tabular}{|c|c|c|c|c|c|c|c|}
\hline \multirow[b]{2}{*}{ Stratum } & \multicolumn{4}{|c|}{ Total Number } & \multicolumn{3}{|c|}{ Return rate in each stratum } \\
\hline & $\begin{array}{c}\text { Paper- } \\
\text { based } \\
\text { survey } \\
\text { sent }\end{array}$ & $\begin{array}{l}\text { Paper- } \\
\text { based } \\
\text { survey } \\
\text { returned }\end{array}$ & $\begin{array}{l}\text { Online } \\
\text { survey } \\
\text { returned }\end{array}$ & $\begin{array}{l}\text { Paper- } \\
\text { based+online } \\
\text { survey } \\
\text { returned } \\
\text { (c+d) }\end{array}$ & $\begin{array}{l}\text { Paper-based } \\
\text { return rate of } \\
\text { the total } \\
\text { number of } \\
\text { the paper- } \\
\text { based sent } \\
\text { (c/b x 100) }\end{array}$ & $\begin{array}{l}\text { Paper-based } \\
\text { return rate of } \\
\text { the total number } \\
\text { of the survey } \\
\text { returned (paper- } \\
\text { based+online) } \\
\text { (cle } \times 100 \text { ) }\end{array}$ & $\begin{array}{l}\text { Online return } \\
\text { rate of the total } \\
\text { number of the } \\
\text { survey returned } \\
\text { (paper- } \\
\text { based+online) } \\
\text { (d/e } \times 100)\end{array}$ \\
\hline a & b & C & d & e & $f$ & $g$ & $\mathrm{~h}$ \\
\hline Researchers & 198 & 111 & 16 & 127 & $56.06 \%$ & $87.40 \%$ & $12.60 \%$ \\
\hline Research assistants & 173 & 79 & 3 & 82 & $45.66 \%$ & $96.34 \%$ & $3.66 \%$ \\
\hline Managers & 72 & 47 & 10 & 57 & $65.28 \%$ & $82.46 \%$ & $17.54 \%$ \\
\hline General employees & 229 & 139 & 17 & 156 & $60.70 \%$ & $89.10 \%$ & $10.90 \%$ \\
\hline TOTAL & 672 & 376 & 46 & 422 & $55.95 \%$ & $89.10 \%$ & $10.90 \%$ \\
\hline
\end{tabular}




\section{Total Number of Survey Sent and Survey Returned}

- Total number of paper-based survey sent

Total number of paper-based survey returned

- Total number of online survey returned

- Total number of paper-based+online survey returned

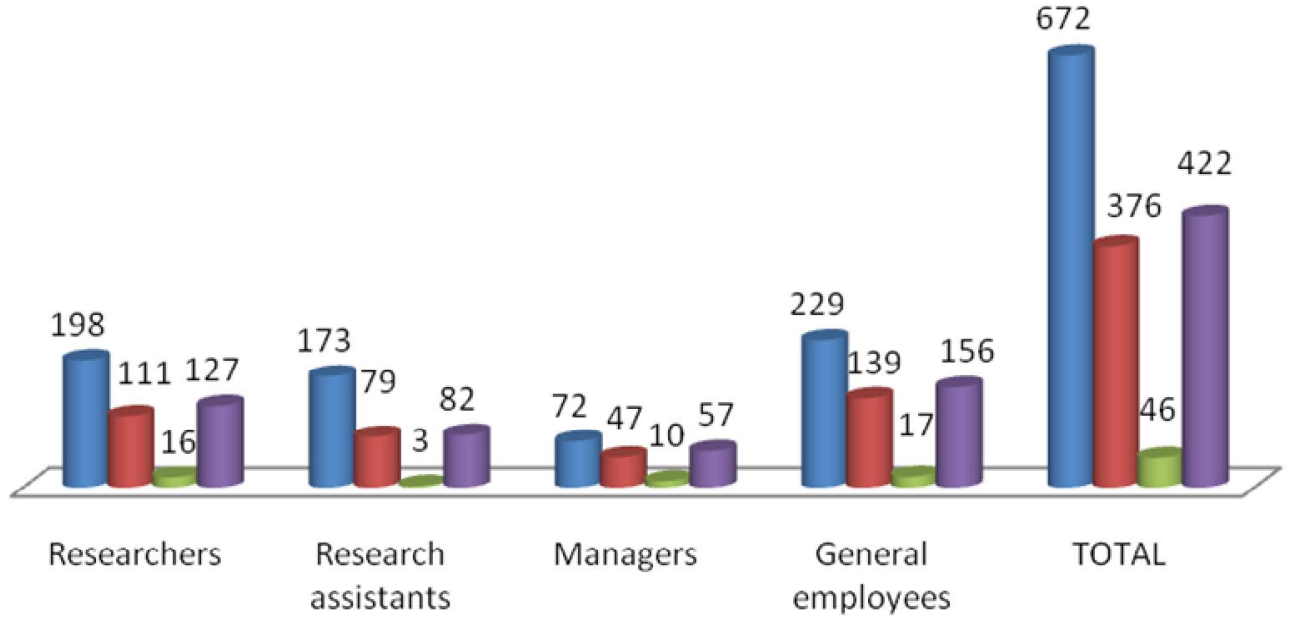

Figure 9: The total number of survey sent and survey returned

\section{Survey Return Rate in each stratum (\%)}

\footnotetext{
Eaper-based return rate of the total number of the paper-based sent in each stratum

a Paper-based return rate of the total number of the survey returned (paper-based + online) in each stratum

Online return rate of the total number of the survey returned (paper-based+online) in each stratum
}

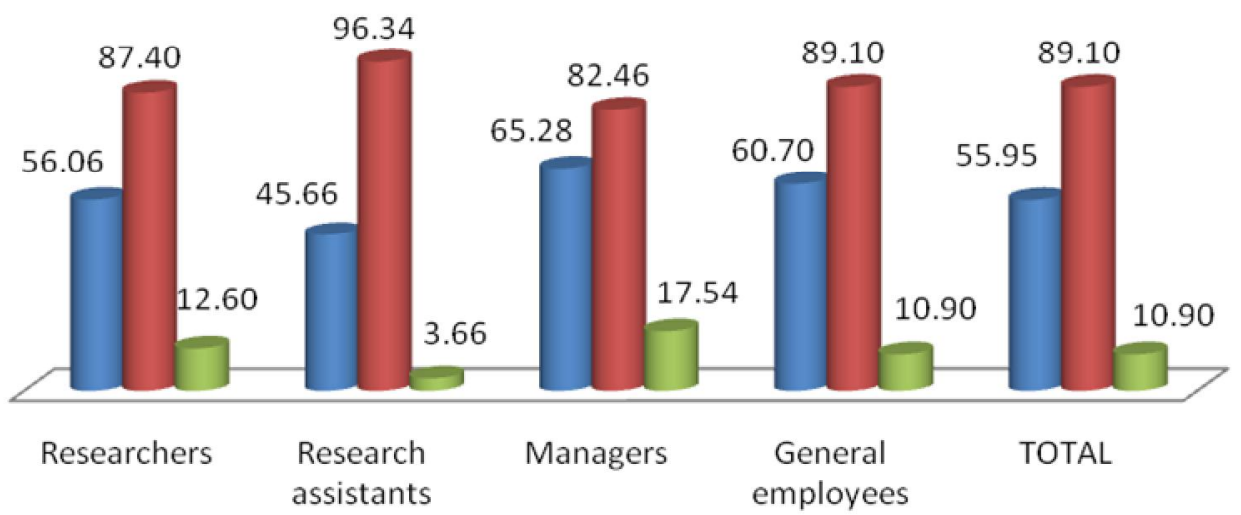

Figure 10: The return rate of the survey in each stratum

Distributing, calculating, and rating the online surveys was quite problematic. The online survey link was distributed to the same groups in all regional offices that received paper-based surveys. Besides including the link within the survey kits, email 
invitations were sent to all 20 FORDA offices. Some surveys were delivered to forestry directors' email addresses and several office email addresses. Additionally, about 20 individuals from all groups (researchers, research assistants, managers, and general employees) had been emailed to distribute the link to their colleagues. A problem of duplication then occurred. Although the information sheet explains that a respondent can choose to submit the survey online or on paper, several respondents submitted both ways. In this research, the online surveys submissions were used, and the paper-based duplicates were removed. This indicates that once the online surveys were delivered, the researcher could not fully control their distribution. For future research it is vital to emphasise that a respondent can only submit one form of survey, either online or paperbased.

From the 40 email invitations sent, 46 participants submitted online. It appears that managers have the highest paper-based return rate, followed by general employees, researchers, and research assistants at $65.28 \%, 60.70 \%, 56.06 \%$, and $45.66 \%$, respectively (Figure 10).

Although the results only show a small proportion of online submission, it appears that managers had the highest proportion of online submission (17.54\%), followed by researchers $(12.60 \%)$, and general employees $(10.90 \%)$. Conversely, research assistants had the lowest proportion of online submission (3.66\%). This indicates that managers are more exposed to the online environment compared to other groups. The proportion of online submission of each group can be taken into consideration while developing and implementing OES at FORDA.

Table 19 presents the sample characteristics. Most (70\%) of the respondents were males. Nearly $50 \%$ of the respondents were between 26 and 35 years old. The proportions of respondents' highest qualification were: $24 \%$ had postgraduate degrees; 53.5\% had Bachelors and Diplomas degrees; and 22.6\% had high school or lower qualifications. Almost all managers and researchers had tertiary level qualifications. Nearly half of these qualifications are at a postgraduate level. Over 50\% of IT personnel/research assistants and about a third of general employees did not have a tertiary level qualification. 
Table 19. Sample characteristics

\begin{tabular}{|c|c|c|c|}
\hline No. & Descriptions & $\begin{array}{l}\text { Frequency } \\
\text { (N) }\end{array}$ & $\begin{array}{c}\text { Percentage } \\
(\%)\end{array}$ \\
\hline \multirow[t]{3}{*}{1.} & Gender: & & \\
\hline & Male & 204 & 70.8 \\
\hline & Female & 84 & 29.2 \\
\hline \multirow[t]{5}{*}{2.} & Age groups: & & \\
\hline & $18-25$ & 22 & 7.6 \\
\hline & $26-35$ & 140 & 48.6 \\
\hline & $36-45$ & 68 & 23.6 \\
\hline & $46-55$ & 58 & 20.1 \\
\hline \multirow[t]{4}{*}{3.} & Education: & & \\
\hline & High school or lower & 65 & 22.6 \\
\hline & Bachelors degrees and Diplomas & 154 & 53.5 \\
\hline & Postgraduates & 69 & 24.0 \\
\hline \multirow[t]{3}{*}{4.} & Having Internet access at work (B1atwork) & & \\
\hline & Yes & 250 & 86.8 \\
\hline & No & 38 & 13.2 \\
\hline \multirow[t]{3}{*}{5.} & Having Internet access at home (B2athome) & & \\
\hline & Yes & 56 & 19.4 \\
\hline & No & 232 & 80.6 \\
\hline \multirow[t]{5}{*}{6.} & Job title: & & \\
\hline & Manager & 42 & 14.6 \\
\hline & IT professional and research assistant * & 55 & 19.1 \\
\hline & Researcher & 91 & 31.6 \\
\hline & General employee & 100 & 34.7 \\
\hline
\end{tabular}

* Of the 288 respondents, there are only three IT personnel. As the number of IT personnel is too small, they were included in the research assistant group. Therefore, it is important to treat the data interpretation carefully as research assistants may have different levels of education compared to IT personnel and may be less familiar with online technology. It is also important to note that some job descriptions do not require employees to work or to be involved in an online environment. Some of them may never need to use such online technology to accomplish their tasks.

Nearly $90 \%$ of respondents have Internet access at work; whilst only about $20 \%$ have Internet access at home. About $60 \%$ of respondents who have Internet access at home are researchers and managers. Almost all managers, researchers, and IT personnel/research assistants have an Internet connection at work; whilst only a few general employees have Internet access at work.

\subsection{Step One: Assessing internal consistency}

This section explains the first step of the quantitative data analysis - assessing internal consistency of the eight constructs.

Internal consistency refers to "the degree to which the items that make up the scale 'hang together' " (Pallant, 2007, p. 95). The assessment was performed to ensure that all items within the scale are measuring the same underlying construct or to make sure that the scale is reliable (Pallant, 2007, p. 95). 
Cronbach (1951) noted that any research based on measurement must be concerned with the accuracy or dependability or reliability of measurement. He confirmed that a reliability coefficient shows whether the test designer was correct in expecting a certain collection of items to yield interpretable statements about individual differences.

Santos (1999) defined Cronbach's alpha as an index of reliability associated with the variation accounted for by the true score of the underlying construct in which it determines the internal consistency or average correlation of items in a survey instrument to test its reliability. Additionally, Meyer (2009) noted Cronbach's alpha as a scale reliability coefficient assessing the reliability of a rating summarising a group of test or survey answers which measure some underlying factor. The scale is defined by the sum of scores over all the test items: a score is computed from each test item and the overall rating (Meyer, 2009). Subsequently, he defined reliability as the square of the correlation between the measured scale and the underlying factor the scale was supposed to measure. Moreover, UCLA-ATS (2009) defined Cronbach's alpha as a coefficient of reliability or consistency measuring how well a set of items/variables measures a single unidimensional or multidimensional underlying construct. The formula for the standardised Cronbach's alpha coefficient is (UCLA-ATS, 2009):

$$
a=\frac{N \cdot \bar{c}}{\bar{v}+(N-1) \cdot \bar{c}}
$$

$\alpha=$ Cronbach's alpha coefficient

$\mathrm{N}=$ the number of items

c-bar $=$ the average inter-item covariance among the items

$\mathrm{v}$-bar $=$ the average variance

If the number of items increases, Cronbach's alpha increases; if the average inter-item correlation is low, alpha will be low; furthermore as the average inter-item correlation increases, Cronbach's alpha increases (UCLA-ATS, 2009). Therefore, if the inter-item correlations are high, this implies that the items are measuring the same underlying construct which means that they have "high" or "good" reliability (UCLA-ATS, 2009). An ideal $\alpha$ for a scale would be above 0.7 (Pallant, 2007, p. 95). The reliability of the eight scales in this study was tested by using Cronbach's Alpha statistic in four stages. Table 20 presents the results of the internal consistency tests in four stages. 
Table 20. Four stages of factor analysis

\begin{tabular}{|c|c|c|c|c|c|}
\hline \multirow[t]{2}{*}{ Dimensions } & \multirow[t]{2}{*}{ Scale name } & \multicolumn{4}{|c|}{$\begin{array}{c}\text { Cronbach's Alpha coefficient in each } \\
\text { stage }\end{array}$} \\
\hline & & 1 & 2 & 3 & 4 \\
\hline I. Technological skill readiness & TOTAL I & 0.927 & 0.927 & - & 0.927 \\
\hline II. Online learning style readiness & & 0.576 & 0.639 & 0.725 & - \\
\hline (1) TOTAL Ila - positive online learning style readiness & TOTAL Ila & - & - & - & 0.725 \\
\hline (2) TOTAL Ilb - negative online learning style readiness & TOTAL Ilb & - & - & - & 0.534 \\
\hline III. Equipment/infrastructure readiness & TOTAL III & 0.774 & 0.774 & - & - \\
\hline (1) Component $1-$ Hardware and software adequacy & & - & - & - & 0.849 \\
\hline (2) Component 2 - Network/Internet usage & & - & - & - & 0.784 \\
\hline (3) Component 3 - Other infrastructure support & & - & - & - & 0.828 \\
\hline IV. Attitude readiness & TOTAL IV & 0.899 & 0.748 & 0.886 & - \\
\hline (1) Component 1 - Confidence in the benefits of e-learning & & - & - & - & 0.881 \\
\hline (2) Component 2 - Confidence in coping with online environment & & - & - & - & 0.806 \\
\hline (3) Component $3-$ Fear of e-learning usage & & - & - & - & 0.757 \\
\hline (4) Component 4 - Motivation & & - & - & - & 0.732 \\
\hline V. Human resource readiness & TOTAL V & 0.865 & 0.865 & - & - \\
\hline (1)Component 1 - Personnel & & - & - & - & 0.901 \\
\hline (2)Component 2 - Management & & - & - & - & 0.807 \\
\hline VI. Environmental readiness & TOTAL VI & 0.063 & 0.464 & 0.335 & \\
\hline (1) Component 1 - Negative & & - & - & - & 0.565 \\
\hline (2) Component 2 - Positive & & - & - & - & 0.335 \\
\hline VII. Cultural readiness & & 0.439 & 0.674 & 0.735 & \\
\hline (1)TOTAL Vlla positive cultural readiness & TOTAL VIIa & - & - & - & 0.735 \\
\hline (2)TOTAL VIlb negative cultural readiness & TOTAL VIIb & & & & 0.735 \\
\hline VIII. Financial readiness & & 0.626 & 0.626 & - & - \\
\hline (1)TOTAL VIIIa - Financial affordability & TOTAL VIIIa & - & - & - & 0.729 \\
\hline (2) TOTAL VIIIb - Financial availability & TOTAL VIIIb & - & - & - & 0.389 \\
\hline (3) TOTAL VIIIc - Financial possibility & TOTAL VIIIC & - & - & - & 0.796 \\
\hline
\end{tabular}

Column 1 = Stage 1 - all negative items reversed "Strongly agree" = 1; "Strongly disagree" = 5

Column 2 = Stage 2 - all items use original values "Strongly agree" = 5; "Strongly disagree" = 1 (all negative items were not reversed)

Column 3 = Stage 3 - negative items removed

Column 4 = Stage 4 - items separated into different scales as suggested by factor analysis

\subsubsection{Stage 1}

There are twelve negatively worded questions: Dimension II (CII5, CII7, CII8), Dimension IV (CIV2, CIV5, CIV13, CIV22, CIV23), Dimension VI (CVI2, CVI3), and Dimension VII (CVII3, CVII4). One question (CI7) was deleted as it was incorrectly set up and could not be answered.

The values of all twelve negatively worded questions were reversed from "Strongly agree" $=1$ to "Strongly disagree" $=5$. The first reliability test was conducted on all dimensions. The results show that Dimensions I, III, IV, and V have acceptable $\alpha$ (above 0.7). Conversely, Dimensions VIII, II and VII have low $\alpha$ (below 0.7), and Dimension VI has a very low $\alpha$ at 0.063 . Appendix 2 presents detailed information about the first test.

\subsubsection{Stage 2}

The second reliability test was performed to check whether respondents had mistakenly answered the negatively worded questions while completing the survey, particularly in Dimensions II, VI, and VII which had low $\alpha$. Therefore, in this second test, the rest of 
dimensions including those which do not have negative items and those which have negative items but have acceptable $\alpha$ were not discussed.

The second test was conducted by setting back the values of all the twelve negatively worded items to the initial values from "Strongly agree" $=5$ to "Strongly disagree" $=1$. The results from the second test show that $\alpha$ increased in Dimension II (from 0.576 to 0.639 ) and Dimension VII (from 0.439 to 0.674 ). A significant increase was also seen in Dimension VI (from 0.063 to 0.464 ). These indicate that some respondents might not have answered the negatively worded questions correctly.

\subsubsection{Stage 3}

Since the results from Stage 2 are below 0.7, a third reliability test was performed involving four dimensions: II (.639), VI (.464), VII (.674), and VIII (.626). The third reliability test was conducted to test whether the reliability of each scale would improve by removing the negatively worded items. Again, other dimensions (Dimensions I, III, IV, and V) with acceptable $\alpha$ values were not discussed further in this stage.

The third test was performed by removing all the negative items in Dimension II, VI, and VII. The results show that the $\alpha$ in Dimension II (.725) and VII (.735) reached acceptable levels. However, $\alpha$ in Dimension VI decreased from .464 to .335 , but this was higher than that of the first test result when all items were included. In this case, a factor analysis was performed to evaluate and test the construct validity of the relationships between items within each of the eight dimensions. The decision was made because the removal of negatively worded items had improved $\alpha$.

\subsubsection{Factor analysis}

It is important to note that, although factor analysis was conducted with all dimensions, only three dimensions will be discussed thoroughly: Dimensions II, VII, and VIII. Additional justifications for this decision will be discussed further, alongside other explanation in this section.

According to Field (2005, p. 731), "factor analysis is a multivariate technique for identifying whether the correlations between a set of observed variables stem from their relationship to one or more latent variables in the data, each of which takes the form of 
a linear model". Although the main reasons for conducting factor analysis are to identify the underlying dimensions and to reduce the dataset to a smaller, more parsimonious set of uncorrelated factors (Field, 2000, p. 423), this study did not reduce the number of items within scales or subscales.

As suggested by Pallant (2007), the factor analysis in this study was conducted in three main parts: (1) assumption checking; (2) factor extraction; and (3) factor rotation and interpretation.

\subsubsection{Part 1: Assumption checking for factor analysis}

To help assess the factorability of the data, SPSS generates two statistical measures: Bartlett's Test of Sphericity and the Kaiser-Meyer-Olkin (KMO) measure of sampling adequacy. Pallant (2007, p. 185) suggested that to be considered appropriate for factor analysis, the correlation matrix should show at least some correlations of $r=0.3$ or greater. Moreover, Bartlett's test of Sphericity should be statistically significant at $\mathrm{p}<$ 0.05 , and the KMO value should be 0.6 or above (Pallant (2007). Appendix 3 presents a more detailed output of factor analysis provided by SPSS.

Table 21 presents the summary of assessment results of the two statistical measures. The results show that eight dimensions had $\mathrm{p}$ values of less than 0.05. Additionally, only six dimensions had KMO values above 0.6. The KMO values in Dimension VI (0.535) and Dimension VIII (0.595) were below the acceptable level. Therefore, only six dimensions met these two criteria and were considered suitable for factor analysis. However, factor analysis was performed on all dimensions. Pallant (2007) added that ideally the sample size should be 150 or more and there should be a ratio of at least five cases for each of the variables. The data in this study have met these criteria.

Table 21. Assumption checking for factor analysis

\begin{tabular}{|l|c|c|c|}
\hline \multicolumn{1}{|c|}{ Dimensions } & Score Range & $\begin{array}{c}\text { Kaiser-Meyer-Olkin } \\
\text { Measure of Sampling } \\
\text { Adequacy (KMO) }\end{array}$ & $\begin{array}{c}\text { Bartlett's Test of } \\
\text { Sphericity } \\
\text { (p value) }\end{array}$ \\
\hline I. Technological skill readiness & 6 to 30 & 0.890 & 0.000 \\
\hline II. Online learning style readiness & 9 to 45 & 0.757 & 0.000 \\
\hline III. Equipment/ infrastructure readiness & 8 to 40 & 0.746 & 0.000 \\
\hline IV. Attitude readiness & 23 to 115 & 0.904 & 0.000 \\
\hline V. Human resource readiness & 11 to 55 & 0.861 & 0.000 \\
\hline VI. Environmental readiness & 4 to 20 & 0.535 & 0.000 \\
\hline VII. Cultural readiness & 6 to 30 & 0.632 & 0.000 \\
\hline VIII. Financial readiness & 7 to 35 & 0.595 & 0.000 \\
\hline
\end{tabular}

Appropriate/ideal for doing factor analysis $=\mathrm{KMO}$ value $\geq 0.6$; and $\mathrm{p}$ value $\leq 0.05$ 
As mentioned before, four dimensions (Dimensions I, III, IV, and V) with acceptable $\alpha$ (the results from Stage 1) will not be discussed at length in this study. Dimension VI did not meet the standard for doing a factor analysis, therefore it will not be discussed either. Although the KMO value in Dimension VIII did not meet the standard, a factor analysis was conducted on this dimension as the KMO value was only slightly below the standard at 0.595 . Therefore, in this stage, only three dimensions which have low $\alpha$ as depicted in the previous stage will be discussed thoroughly: Dimensions II, VII, and VIII. However, in order to give an overview, all results of the extraction suggested by SPSS are presented in Table 22. The justification for breaking up scales in three dimensions is presented in the following section. Appendix 3 presents more detailed information about the factor analysis including the correlation between items within Dimensions II, VII, and VIII.

Table 22. Scale and subscales after factor analysis

\begin{tabular}{|c|c|c|}
\hline Dimensions & Factor extraction & $\begin{array}{l}\text { PVC } \\
(\%)\end{array}$ \\
\hline $\begin{array}{l}\text { I. Technological skill } \\
\text { readiness }\end{array}$ & No suggestion for breaking the scale into parts & 73.713 \\
\hline \multirow{2}{*}{$\begin{array}{l}\text { II. Online learning style } \\
\text { readiness }\end{array}$} & (1) Component 1 - Positive online learning style (items: 1, 2, 3, 4, 6, 9); & 29.871 \\
\hline & (2) Component 2 - Negative online learning style (items: 5,7,8) & 22.645 \\
\hline \multirow{3}{*}{$\begin{array}{l}\text { III. Equipment/ } \\
\text { infrastructure } \\
\text { readiness }\end{array}$} & (1) Component 1 - Hardware and software adequacy (items: 1, 2, 3); & 29.292 \\
\hline & (2) Component 2 - Network/ Internet usage (items: 4,5); & 28.172 \\
\hline & (3) Component 3 - Other infrastructure support (items: $6,7,8$ ) & 20.535 \\
\hline \multirow[t]{4}{*}{ IV. Attitude readiness } & (1) Component 1 - Confidence in the benefits of e-learning (items: 18, 19, 6, 12, 17, 4, 20, 13, 11) & 22.430 \\
\hline & (2) Component 2 - Confidence in coping with online environment (items: $9,8,3,10,7,21,1$ ) & 13.599 \\
\hline & (3) Component 3 - Fear of e-learning usage (item 5, 22, 23, 2) & 11.098 \\
\hline & (4) Component 4 - Motivation (items: $15,16,14$ ) & 9.286 \\
\hline \multirow{2}{*}{$\begin{array}{l}\text { V. Human resource } \\
\text { readiness }\end{array}$} & (1) Component 1 - Personnel (items: 10, 8, 9, 6, 7, 5, 11) & 40.117 \\
\hline & (2) Component 2 - Management (items: 2, 3, 1, 4) & 23.650 \\
\hline \multirow{2}{*}{$\begin{array}{l}\text { VI. Environmental } \\
\text { readiness }\end{array}$} & (1) Component 1 - Negative (items: 2, 3) & 34.641 \\
\hline & (2) Component 2 - Positive (items: 1, 4) & 31.220 \\
\hline \multirow[t]{2}{*}{ VII. Cultural readiness } & (1) Component 1 - E-learning culture - positive cultural readiness (items: 1, 2, 5, 6) & 36.759 \\
\hline & (2) Component 2 - Conventional culture - negative cultural readiness (items: 3,4 ) & 29.157 \\
\hline \multirow[t]{3}{*}{ VIII. Financial readiness } & (1) Component 1 - Financial affordability (items: 1, 2, 3) & 28.357 \\
\hline & (2) Component 2 - Financial availability (items: 4, 5) & 23.795 \\
\hline & (3) Component 3 - Financial investment possibility (items: 6,7) & 18.135 \\
\hline
\end{tabular}

PVC = Percent of the variance of each scale - contribution \%

Dimensions that were split in this study after factor analysis are in bold

\subsubsection{Part 2: Factor extraction}

Pallant (2007, p. 181) noted that "Factor extraction involves determining the smallest number of factors that can be used to best represent the interrelations among the set of variables". Of the various techniques available, this study employed component analysis, the most commonly used approach to identify (extract) the number of underlying factors (Pallant, 2007, p. 182). Two procedures from this approach were selected to assist in the decision making of identifying (extracting) the number of 
underlying factors: Kaiser's criterion or eigenvalue rule and Cattel's scree test. These two procedures were provided by SPSS.

Pallant (2007, p. 182) noted that "The eigenvalue of a factor represents the amount of the total variance explained by that factor". Furthermore, Pallant (2007, p. 182) and Field (2000, p. 436) suggested that only factors with an eigenvalue of 1.0 or more can be retained for further examination. Therefore, in this study, factors that have an eigenvalue of 1.0 or above will be retained for further examination. Table 23 presents the summary of Kaiser's criterion and the percentage of total cumulative components retained in each dimension.

Table 23. Deciding factor extraction

\begin{tabular}{|c|c|c|c|c|c|}
\hline \multirow[b]{2}{*}{ Dimensions } & \multirow[b]{2}{*}{$\begin{array}{l}\text { Eigenvalue } \\
\text { rule (E) }\end{array}$} & \multicolumn{3}{|c|}{ Initial Eigenvalues } & \multirow{2}{*}{$\begin{array}{c}\text { Total } \\
\text { Cumulative } \\
(\%)\end{array}$} \\
\hline & & Total & $\begin{array}{c}\% \text { of } \\
\text { Variance }\end{array}$ & $\begin{array}{c}\text { Cumulative } \\
\%\end{array}$ & \\
\hline A & B & C & D & $E$ & $\mathrm{~F}$ \\
\hline I. Technological skill readiness & 1 & * & * & * & 73.713 \\
\hline II. Online learning style readiness & 2 & $\begin{array}{l}2.722 \\
2.004\end{array}$ & $\begin{array}{l}30.248 \\
22.268\end{array}$ & $\begin{array}{l}30.248 \\
52.517\end{array}$ & 52.517 \\
\hline III. Equipment/ infrastructure readiness & 3 & * & * & * & 77.999 \\
\hline IV. Attitude readiness & 4 & * & * & * & 56.413 \\
\hline V. Human resource readiness & 2 & * & * & * & 63.767 \\
\hline VI. Environmental readiness & 2 & $\star \star$ & $\star \star$ & ** & 65.851 \\
\hline VII. Cultural readiness & 2 & $\begin{array}{l}2.419 \\
1.536\end{array}$ & $\begin{array}{l}40.309 \\
25.608\end{array}$ & $\begin{array}{l}40.309 \\
65.917\end{array}$ & 65.917 \\
\hline VIII. Financial readiness & 3 & $\begin{array}{l}2.256 \\
1.657 \\
1.007\end{array}$ & $\begin{array}{l}32.225 \\
23.670 \\
14.392\end{array}$ & $\begin{array}{l}32.225 \\
55.895 \\
70.287\end{array}$ & 70.287 \\
\hline
\end{tabular}

The second procedure for factor retention was Cattel's scree test (Pallant, 2007, p.182) involving plotting each of the eigenvalues of the factor and inspecting the plot to locate a point at which the shape of the curve changes direction and becomes flat. Pallant (2007, p. 182) suggested retaining all factors above the elbow (a change in the shape of the plot), or breaking the plot, because these factors contribute the most to the explanation of the variance in the data set.

Dimension II has two components with an eigenvalue above 1 (Table 23 Column C). These two components explain a total of $52.517 \%$ of the variance (Table 23 Column F). The Screeplot in Figure 11 also shows that two components were above the elbow. Thus, the researcher considered retaining two components in Dimension II for further investigation. 


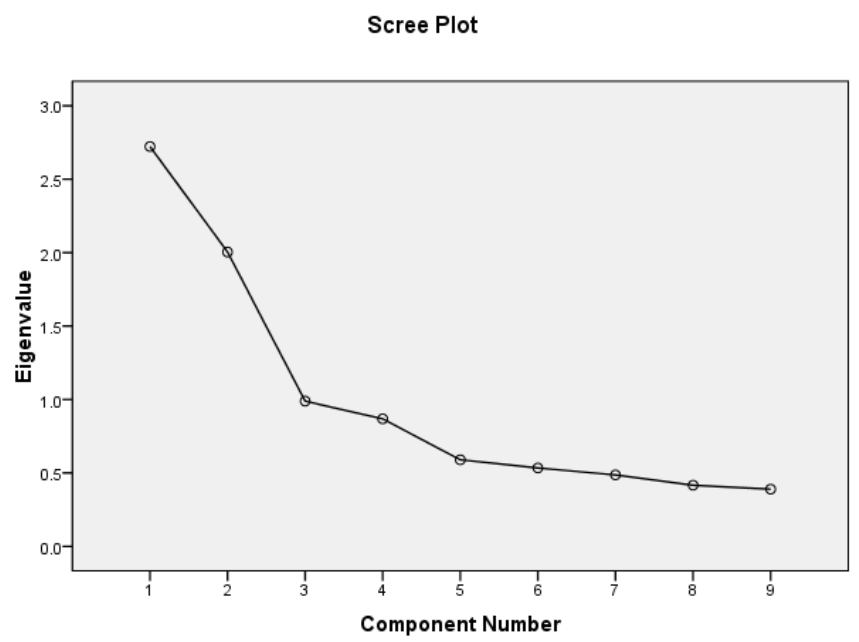

Figure 11: Screeplot - Dimension II

Dimension VII has two components with an eigenvalue above 1 (Table 23 Column C), and these two components explain a total of $65.917 \%$ of the variance. The Screeplot shows a clear break after the second component (Figure 12). Thus, the researcher considered extracting two components in Dimension VII for further investigation.

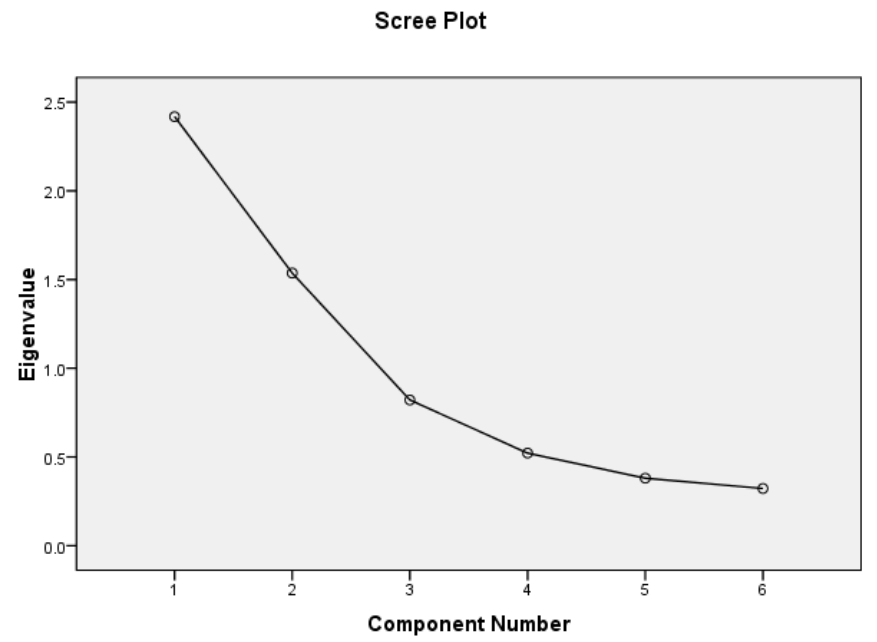

Figure 12: Screeplot - Dimension VII

Dimension VIII has three components with an eigenvalue above 1 (Table 23 Column C), and these three components explain a total of $70.287 \%$ of the variance. The Screeplot also revealed a clear break after three components (Figure 13). Thus, the researcher considered extracting three components in Dimension VIII for further examination. 


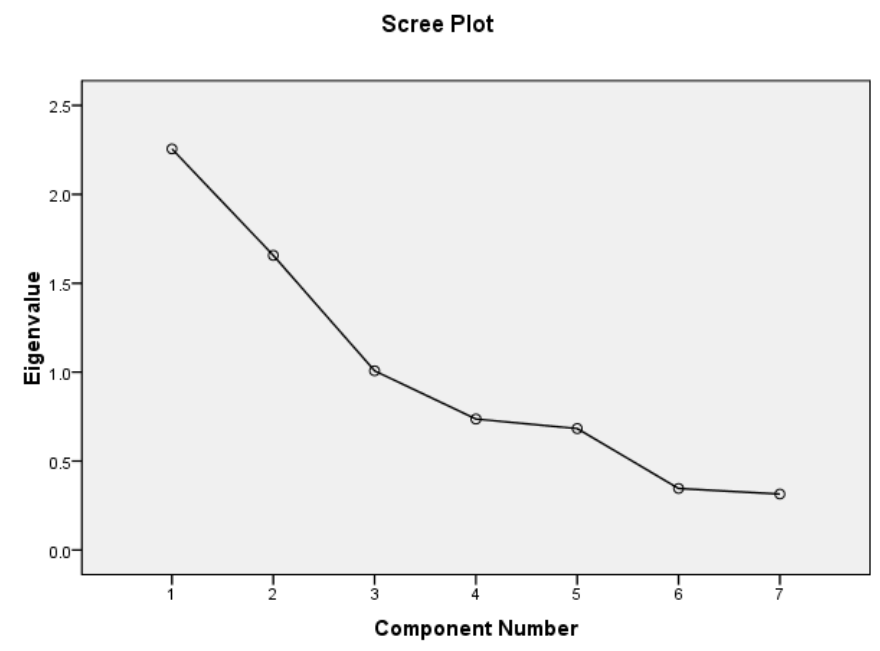

Figure 13: Screeplot - Dimension VIII

In this study the factorability of the data was assessed by using two approaches: Kaiser's criterion and scree tests. It would be better for future research to include Horn's parallel analysis (Horn, 1967) which can generate a more accurate approach in identifying the correct number of components retained as suggested by Pallant (2007).

\subsubsection{Part 3: Factor rotation and interpretation}

After determining the number of components (factors), the factors were rotated to presents the pattern of loadings in a manner that is easier to interpret. The rotation method used in this study was Varimax with Kaiser Normalisation which is provided by SPSS. Pallant (2007, p. 183) noted that the Varimax technique attempts to minimise the number of variables that have high loadings on each factor. Pallant (2007) also noted that SPSS does not label or interpret each of the factors; it only reveals which variables cluster together. Thus the researcher has to decide where to break up the scales.

\subsection{Dimension II}

The factor loadings before and after rotation for Dimension II are presented in Table 24. After factor rotation, the factors in Dimension II cluster together in two components as suggested by SPSS: Component 1 (items CII2, CII4, CII1, CII3, and CII6) and Component 2 (CII7, CII8, CII9, and CII5). In Appendix 3, a Table Component Transformation Matrix presents the correlations between components. The strength of the relationship between the two components was quite low at .217: a small correlation is if $\mathrm{r}= \pm .10$ to \pm .29 (Cohen, 1992, p. 99). 
Table 24. Factor loadings - Dimension II before and after rotation

\begin{tabular}{|c|c|c|c|c|}
\hline & \multicolumn{2}{|c|}{ Component Matrix a } & \multicolumn{2}{|c|}{ Rotated Component Matrix } \\
\hline & 1 & 2 & 1 & 2 \\
\hline $\mathrm{Cll} 2^{*}$ & .811 & & .828 & \\
\hline $\mathrm{Cll} 4^{*}$ & .786 & & .796 & \\
\hline $\mathrm{ClI} 1^{*}$ & .780 & & .795 & \\
\hline $\mathrm{Cll} 3^{*}$ & .682 & & .682 & \\
\hline $\mathrm{Cll} 6^{*}$ & .419 & & .464 & \\
\hline $\mathrm{Cll} 7^{\star \star}$ & & .818 & & .827 \\
\hline $\mathrm{Cll} 8^{\star *}$ & & .778 & & .811 \\
\hline $\mathrm{Cll9* \star}$ & .348 & -.686 & & -.746 \\
\hline $\mathrm{Cll5} 5^{\star *}$ & & .346 & & .327 \\
\hline
\end{tabular}

a Extraction Method: Principal Component Analysis. Two components extracted.

b Extraction Method: Principal Component Analysis. Rotation Method: Varimax with Kaiser Normalisation.

Rotation converged in three iterations.

* Component 1

** Component 2

Pallant (2007) suggested that a communalities value below .3 is considered low, indicating that the item does not fit well with the other items in its component. The results show that items CII5 and CII6 have low communalities values at .122 and .240 respectively (Appendix 3 Table Communalities) indicating that that these items do not fit well with the other items in their component. Additionally, item CII5 has the lowest loading on Component 2 at .346 (Appendix 3 Table Rotated Component Matrix). Thus, for future researchers may consider removing this item to improve the scale.

Based on this information provided by SPSS, the researcher decided to break the scale into two subscales. However, as noted by Pallant, the SPSS cannot interpret the results. The researcher decided to put item CII9 in Component 1 instead of Component 2 because the question tended to be positive towards online learning style. Accordingly, Component 1 includes items CII2, CII4, CII1, CII3, CII6, and CII9; whilst Component 2 includes items CII7, CII8, and CII5.

\subsection{Dimension VII}

The factor loadings before and after rotation for Dimension VII are presented in Table 25. After factor rotation, the factors in Dimension VII cluster together in two components as suggested by SPSS: Component 1 (CVII5, CVII2, CVII1) and Component 2 (CVII3, and CVII4). Therefore, the scale was split into two components. The strength of the relationship between these two components was medium at .491: a medium correlation is if $\mathrm{r}= \pm .30$ to \pm .49 (Cohen, 1992, p. 99) (Appendix 3 Table Component Transformation Matrix). All items have communalities values above .3 (Appendix 3 Table Communalities) indicating that that all items fit well with the other items in their component. Therefore, the researcher decided to break Dimension VII into these two components as suggested by SPSS. 
Table 25. Factor loadings - Dimension VII before and after rotation

\begin{tabular}{|l|c|c|c|c|}
\hline \multirow{2}{*}{} & \multicolumn{2}{|c|}{ Component Matrix ${ }^{\mathrm{a}}$} & \multicolumn{2}{c|}{ Rotated Component Matrix $^{\mathrm{b}}$} \\
\cline { 2 - 5 } & $\mathbf{1}$ & $\mathbf{2}$ & $\mathbf{1}$ & $\mathbf{2}$ \\
\hline CVII5* $^{*}$ & .771 & & .674 & -.374 \\
\cline { 2 - 5 } CVII2 $^{*}$ & .695 & .460 & .831 & \\
CVII1* $^{*}$ & .680 & .523 & .849 & \\
\cline { 2 - 5 } CVII6* $^{*}$ & .673 & & .574 & -.352 \\
\hline CVIII $^{* *}$ & -.413 & .753 & & .859 \\
\cline { 2 - 5 } CVIII $^{* *}$ & -.506 & .695 & & .854 \\
\hline
\end{tabular}

a Extraction Method: Principal Component Analysis. Two components extracted.

b Extraction Method: Principal Component Analysis. Rotation Method: Varimax with Kaiser Normalisation.

Rotation converged in three iterations.

* Component 1

** Component 2

\subsection{Dimension VIII}

The factor loadings before and after rotation for Dimension VIII are presented in Table 26. After factor rotation, the factors in Dimension VIII cluster together in three components as suggested by SPSS: Component 1 (CVIII1, CVIII2, CVII3); Component 2 (CVIII6 and CVIII7); and Component 3(CVIII4 and CVIII5). Component 2 has weak relationships with other components. There is a medium relationship between Component 1 and Component 3. The strength of the relationship between these three components is presented in Table 27 Component Transformation Matrix. All items have communalities values above .3, (Appendix 3 Table Communalities) indicating that all items fit well with the other items in the component. Based on this information, the researcher decided to break Dimension VIII into three components as suggested by SPSS.

Table 26. Factor loadings - Dimension VIII before and after rotation

\begin{tabular}{|c|c|c|c|c|c|c|}
\hline & \multicolumn{3}{|c|}{ Component Matrix $^{\text {a }}$} & \multicolumn{3}{|c|}{ Rotated Component Matrix ${ }^{b}$} \\
\hline & 1 & 2 & 3 & 1 & 2 & 3 \\
\hline CVIII2* & .819 & -.038 & -.317 & .873 & .055 & .093 \\
\hline $\mathrm{CVIII}^{*}$ & .778 & -.059 & -.123 & .749 & .037 & 249 \\
\hline $\mathrm{CVIIII}^{*}$ & .674 & -.100 & -.308 & .746 & & .041 \\
\hline CVIIII*** & .563 & -.145 & .433 & .311 & -.051 & .653 \\
\hline CVIIII** & .116 & .901 & .125 & -.040 & .913 & .074 \\
\hline CVIII6* & .157 & .899 & -.070 & .086 & .907 & -.080 \\
\hline 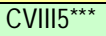 & .412 & -.049 & .767 & & .039 & .871 \\
\hline
\end{tabular}

a Extraction Method: Principal Component Analysis. Three components extracted.

b Extraction Method: Principal Component Analysis. Rotation Method: Varimax with Kaiser Normalisation. Rotation converged in four iterations.

* Component 1

** Component 2

*** Component 3

Table 27. Component Transformation Matrix

\begin{tabular}{|c|c|c|c|}
\hline Component & $\mathbf{1}$ & $\mathbf{2}$ & $\mathbf{3}$ \\
\hline $\mathbf{1}$ & .882 & .130 & .453 \\
\hline $\mathbf{2}$ & -.095 & .991 & -.099 \\
\hline $\mathbf{3}$ & -.461 & .045 & .886 \\
\hline
\end{tabular}




\subsubsection{Stage 4}

The fourth reliability test was conducted to check all dimensions after being broken down by factor analysis. Appendix 4 presents detailed information about the fourth test. As mentioned above, the results from Stage 1 show ideal $\alpha$ in Dimensions I (0.927), III (0.774), IV (0.899), and V (0.865); thus, the researcher decided to use these original four scales from the Stage 1 test. Although Dimension VI had a very low $\alpha(0.063)$ in Stage 1, the researcher also decided to use the initial Dimension VI. The decision was made because the number of items in this scale was too small. Moreover, Dimension VI did not meet the criteria for conducting factor analysis.

For the next regression analysis, the researcher decided to use these five subscales as suggested by factor analysis because they had acceptable $\alpha$ : TOTALs IIa (0.725), VIIa (0.735), VIIb (0.735), VIIIa (0.729), and VIIIc (0.796). Consequently two subscales were also used although they had low $\alpha$ : IIb (0.534) and VIIIb (0.389). Table 28 presents the final results of reliability tests that were going to be used for the multiple regression analysis which is discussed in the next section. In the final stage, there are twelve scales within the COERI: TOTALs I, IIa, IIb, III, IV, V, VI, VIIa, VIIb, VIIIa, VIIIb, and VIIIc (Figure 14).

Table 28. Summary of the reliability results

\begin{tabular}{|c|c|c|c|}
\hline Dimensions & Scale name & $\begin{array}{c}\text { Cronbach's } \\
\text { Alpha } \\
\text { coefficient }\end{array}$ & Item descriptions \\
\hline 1. Technological skill readiness & TOTAL I & 0.927 & $\mathrm{Cl} 1$ - Cl6; range 6 to 30 \\
\hline 2. Positive online learning style readiness & TOTAL IIa & 0.725 & Cll1, Cll2, Cll3, Cll4, Cll6, \& Cll9; range: 6 to 30 \\
\hline 3. Negative online learning style readiness & TOTAL IIb & 0.534 & Cll5, Cll7, \& Cll8; range 3 to 15 \\
\hline 4. Equipment/infrastructure readiness & TOTAL III & 0.774 & CIII1 - Clll8; range 8 to 40 \\
\hline 5. Attitude readiness & TOTAL IV & 0.899 & CIV1 - CIV23; range 23 to 115 \\
\hline 6. Human resource readiness & TOTAL V & 0.865 & CV1 - CV11; range 11 to 55 \\
\hline 7. Environmental readiness & TOTAL VI & 0.063 & CVI1 - CVI4; range 4 to 20 \\
\hline 8. $\quad$ Positive cultural readiness & TOTAL VIIa & 0.735 & CVII1, CVII2, CVII5, \& CVII6; range 4 to 20 \\
\hline 9. Negative cultural readiness & TOTAL VIIb & 0.735 & CVII3 \& CVII4; range 2 to 10 \\
\hline 10. Financial affordability & TOTAL VIIIa & 0.729 & CVIII1, CVIII2, \& CVIII3; range 3 to 15 \\
\hline 11. Financial availability & TOTAL VIIIb & 0.389 & CVIII4 \& CVIIII; range 2 to 10 \\
\hline 12. Financial possibility & TOTAL VIIIC & 0.796 & CVIII6 \& CVIIII; range 2 to 10 \\
\hline
\end{tabular}




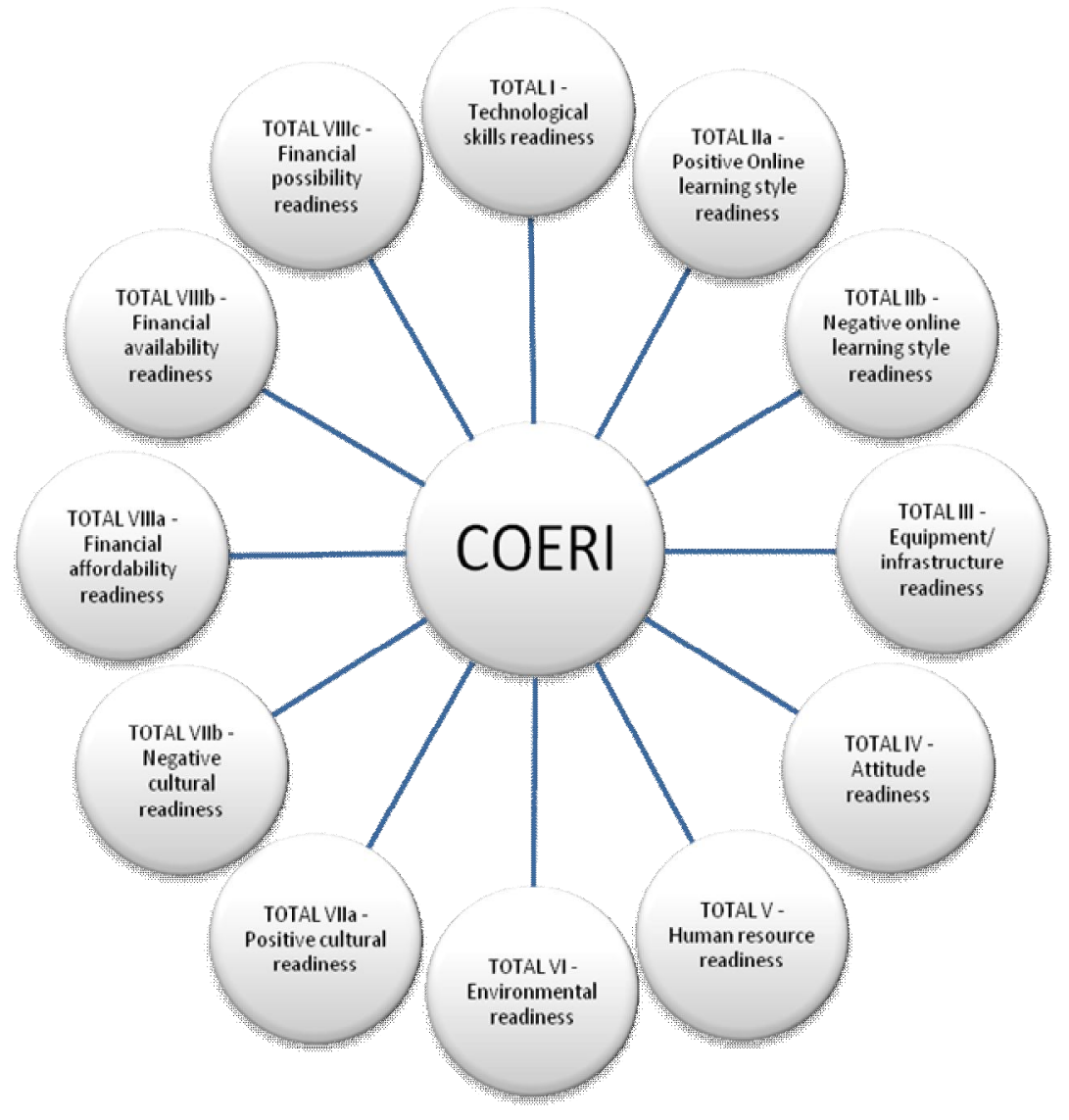

Figure 14: COERI - Twelve dimensions

\subsubsection{Discussion}

It is important to note that in this study, no items were deleted after factor analysis. All items were used for the next multiple regression analysis.

Three scales: TOTALs IIb, VI, and VIIIb require revision as they possess poor reliability. It is necessary to further explore these scales using the ones with better levels of alpha coefficients. Particularly, Dimension VI - environmental readiness needs to be redeveloped and retested to improve its reliability. It might also be significant to explore the level of readiness using the dimensions as suggested by the factor analysis as they possess more reliable results. For example Dimension III - equipment/infrastructure readiness has higher alpha coefficients after being broken down into three subscales.

In addition to that, it is important to note that at the end of the analysis stage, the researcher realised that two items within the TOTAL VIIIc - financial possibility dimension were in fact negatively worded questions: CVIII6 "I think high initial investment may hinder my organisation from developing and implementing e-learning" and CVIII7 "I think high operating costs may hinder my organisation from developing 
and implementing e-learning”. However, the whole analysis used these two items without reversing their values. In this case, the researcher presented the results by reversing the percentage level of readiness which will be discussed further in Section 4.4.12. This indicates that Dimension VIII also needs revision by reversing the score for these two negatively phrased questions.

\subsection{Step Two: Multiple regression analysis and construct evaluation}

This second step explains the multiple regression analysis and the evaluation of the twelve constructs. Multiple regression can be used to address: (1) how well a set of variables is able to predict a particular outcome; (2) which variable in a set of variables is the best predictor of an outcome; and (3) whether a particular predictor variable is still able to predict an outcome when the effects of another variable are controlled for (Pallant, 2007, p. 147). Multiple regression analysis was performed in this study to address how well six independent variables (IVs): age, gender, education, Blatwork (having an Internet connection at work), B2athome (having an Internet connection at home), and job title predict the twelve dependent variables (DVs): scale TOTALs I, IIa, IIb, III, IV, V, VI, VIIa, VIIb, VIIIa, VIIIb and VIIIc.

Before analysing the data, it is important to note that the job title variable was coded into three dummy variables, with general employees as the reference category. The job title variable contained managers, IT personnel/research assistants, researchers, and general employees. The dummy variables are: jobtitle1 (IT personnel/research assistants), jobtitle2 (researchers), and jobtitle3 (managers).

Multiple regression was used to determine whether the six IVs made a significant contribution to the DVs. A standard multiple regression was used in which jobtitle1, jobtitle2, and jobtitle3 were entered into the first block, whilst age, gender, education, Blatwork, and B2athome were entered in the second block of the equation simultaneously. Each IV was evaluated regarding its predictive power, over and above that offered by all the other IVs (Pallant, 2007, p. 147).

\subsubsection{Assumption checking for multiple regression}

The assumptions of no significant outliers, normality of errors, homoscedasticity and independence of errors, and multicollinearity have been checked with no significant 
violation of the assumptions. The assumption checking procedures are available in Appendix 6.

Outliers are cases that have a standardised residual value above 3.3 or below 3.3 (Pallant, 2007, p. 156). Fourteen cases of outliers had been identified in the data. To check that the outliers were within the acceptable distance, Pallant, (2007, p. 157) suggested that the Mahalanobis distance values for the six IVs should be below the critical value of Chi-square for six IVs (22.458). The Mahalanobis distance values were 20.534 which was below the critical value indicating that there is no need to be concerned about these fourteen cases and no need to remove these cases from the data (Pallant, 2007, p. 158).

The assumption of normality of errors has probably been met if the histograms appear to be reasonably normally distributed (Field, 2005, p. 204; Pallant, 2007, p. 149). In addition, most of the Probability Plot of the Regression Residual has to be in a reasonably straight diagonal line from bottom left to top right indicating that there are no major deviations from normality (Field, 2005; Pallant, 2001, p. 137). The results show that these assumptions had probably been satisfied for this study.

The assumptions of homoscedasticity and independence error have probably been met if the variance of the residual about predicted DV scores are the same for all predicted scores (Pallant, 2007, p. 149). Moreover, the scatterplot of the standardised residuals should be roughly rectangularly distributed, with most of the scores concentrated in the centre or along the zero point (Pallant, 2001, p. 156). The Durbin-Watson statistic values should also fall within the recommended boundaries of 1-3, suggesting that errors are reasonably independent indicating that the assumptions of homoscedasticity and independence of errors have been met (Field, 2005, p. 190). The results show that these assumptions have not been violated for the research.

Multicollinearity or a highly correlated relationship among independent variables $(\mathrm{r} \geq .9)$ leads to a poor regression model (Pallant, 2007, p. 149). The assumption of multicollinearity has not been violated if the tolerance values recorded are not near zero (Field, 2005, p. 175; Pallant, 2007, p. 156). Additionally, the variance inflation factor (VIF) value for each independent variable should be less than 10 and the average of VIF values should not be substantially greater than 1 indicating that there is no 
multicollinearity (Field, 2005, p. 175; Pallant, 2007, p. 156). The results show that these assumptions had probably not been violated for this study.

\subsubsection{Evaluation of the constructs}

Table 29 provides the summary of the construct evaluation. Of the twelve DVs, eight scales (TOTALs I, IIa, III, IV, V, VIIa, VIIIa, and VIIIb) reached statistical significance ( $\mathrm{p}<.05)$. TOTAL I has the highest $\mathrm{R}^{2}$ change (percent of variance) at $44.4 \%$, followed by TOTALs IV, III, and IIa indicating that all IVs (age, gender, education, job title, Blatwork, and B2athome) explain $44.4 \%$ of the variance in technological skills readiness; $27.7 \%$ in attitude readiness; $27 \%$ in equipment/infrastructure readiness; and $24.3 \%$ in positive online learning style readiness. Three DVs (TOTALs V, VIIa, and VIIIa) have considerably low $\mathrm{R}^{2}$ change indicating that all IVs explain $19.3 \%$ of the variance in positive cultural readiness; $12.3 \%$ in human resource readiness; and $11.7 \%$ in financial affordability readiness. Five DVs (TOTALs IIb, VI, VIIb, VIIIb, and VIIIc) have a very low $\mathrm{R}^{2}$ change indicating that all IVs only explain $5.4 \%$ of the variance in financial availability readiness; $4.1 \%$ in financial possibility readiness; $2.1 \%$ in negative cultural readiness; and $1.2 \%$ in negative online learning style readiness.

Table 29. Construct evaluation

\begin{tabular}{|c|c|c|c|}
\hline \multirow{2}{*}{$\begin{array}{c}\text { DVs } \\
\text { (Scale) }\end{array}$} & \multirow{2}{*}{$\begin{array}{c}\text { Percent of } \\
\mathrm{R}^{2} \text { change } \\
\text { (\%) }\end{array}$} & \multicolumn{2}{|c|}{ ANOVA - df $(8,279)$} \\
\hline & & $\mathbf{F}$ & $p$ value \\
\hline 1. TOTAL I & 44.4 & 27.810 & 0.000 \\
\hline 2. TOTAL IIa & 24.3 & 11.184 & 0.000 \\
\hline 3. TOTAL IIb & 1.5 & .536 & 0.829 \\
\hline 4. TOTAL III & 27 & 12.895 & 0.000 \\
\hline 5. TOTAL IV & 27.7 & 13.344 & 0.000 \\
\hline 6. TOTAL V & 12.3 & 4.913 & 0.000 \\
\hline 7. TOTAL VI & 4.3 & 1.586 & 0.129 \\
\hline 8. TOTAL VIIa & 19.3 & 8.318 & 0.000 \\
\hline 9. TOTAL VIIb & 2.2 & .772 & 0.627 \\
\hline 10. TOTAL VIIIa & 11.7 & 4.643 & 0.000 \\
\hline 11. TOTAL VIIIb & 5.8 & 2.143 & 0.032 \\
\hline 12. TOTAL VIIIC & 3.6 & 1.300 & 0.243 \\
\hline
\end{tabular}

The influences of IVs on each DV are presented in Section 4.5. A standardised coefficient Beta (ß) depicts which of the IVs give the strongest or the weakest contribution to each DV. A higher $ß$ value indicates a stronger contribution to the DV (by ignoring any negative signs). The strongest predictor has the highest $B$ value. Only significant predictors $(\mathrm{p}<.05)$ are presented in this report. The results of the multiple regression analysis can be seen in Appendix 7. 
The relationships between DVs were analysed using Pearson correlations (r). Pallant (2007, p. 132) noted that the correlation size indicates the strength of the relationship between variables which can range from -1.0 (indicating perfect negative correlation) to 1.0 (indicating perfect positive correlation) and the direction of a linear relationship between two variables. A correlation of 0 indicates that the two variables are uncorrelated or have no linear relationship.

Cohen (1992, p. 99) divided the strength of relationships between variables into three groups: large $(\mathrm{r}= \pm .50$ to \pm 1.0$)$; medium $(\mathrm{r}= \pm .30$ to \pm .49$)$; and small $(\mathrm{r}= \pm .10$ to \pm .29 ). Nine large positive correlations (blue shaded) between DVs were identified in this research. It seems that TOTALs I, IV, and V have strong relationships with a few dimensions. Eighteen positive medium correlations (green shaded) were also identified in this study. TOTALs IIa, III, V, VIIa, and VIIIa have medium relationships with many dimensions. The remaining 38 relationships had small correlations (yellow shaded). Some of these weak relationships were negatively correlated. TOTAL VIIIc had weak relationships with all dimensions. Other dimensions which had small relationships are TOTALs IIb, VI, VIIb, and VIIIb. Furthermore, no linear relationship is exhibited between TOTALs VI and VIIb (pink shaded). Tables 30 and 31 present the results of identified correlations between DVs.

The results of the model evaluation indicate that overall age, gender, education, Internet access at work, Internet access at home, and job title only explained quite low proportions of the variance in all twelve scales. Although they only accounted for quite a small percentage of the variance, they made significant contributions to the scales. Thus, they can still be used to investigate OES readiness, particularly in a developing country context.

The relationships between scales were varied. The majority of relationships between DVs were weak and some had negative correlations. Conversely, nine strong and eighteen medium relationships all had positive correlations. The TOTAL VIIIc scale needs to be explored further as it had weak relationships with all dimensions. TOTAL VI and TOTAL VIIb also need further exploration as they were uncorrelated or had no linear relationship. 
Table 30. Pearson Correlations between twelve scales (dependent variables)

\begin{tabular}{|c|c|c|c|c|c|c|c|c|c|c|c|c|c|}
\hline \multicolumn{2}{|c|}{ TOTAL } & 1 & Ila & Ilb & III & IV & V & VI & VIIa & Vllb & VIIIa & VIIIlb & VIIIC \\
\hline \multirow[t]{4}{*}{ I } & $r$ & 1.000 & $.574^{*+\mathrm{L}}$ & $.038^{\mathrm{S}}$ & $.565^{* * L}$ & $.663^{*+\mathrm{L}}$ & $.535^{* \mathrm{~L}}$ & $.145^{*} \mathrm{~S}$ & $.456^{* * \mathrm{M}}$ & $.157^{\text {*t } \mathrm{S}}$ & $.374^{* * \mathrm{M}}$ & $.228^{* * \mathrm{~S}}$ & $.036^{\mathrm{S}}$ \\
\hline & Sig. & & .000 & .522 & .000 & .000 & .000 & .014 & .000 & .008 & .000 & .000 & .544 \\
\hline & $\mathrm{r}^{2}$ & 1 & 0.329 & 0.001 & 0.319 & 0.440 & 0.286 & 0.021 & 0.208 & 0.025 & 0.140 & 0.052 & 0.001 \\
\hline & PV & 100 & 32.95 & 0.14 & 31.92 & 43.96 & 28.62 & 2.10 & 20.79 & 2.46 & 13.99 & 5.20 & 0.13 \\
\hline \multirow[t]{4}{*}{ Ila } & $r$ & $.574^{*+\mathrm{L}}$ & 1.000 & $-.066^{\mathrm{S}}$ & $.470^{* * \mathrm{M}}$ & $.676^{*+\mathrm{L}}$ & $.446^{* * \mathrm{M}}$ & $.206^{* * S}$ & $.470^{* * \mathrm{M}}$ & $.103^{S}$ & $.370^{+* \mathrm{M}}$ & $.225^{* * \mathrm{~S}}$ & $.005^{\mathrm{S}}$ \\
\hline & Sig. & .000 & & .266 & .000 & .000 & .000 & .000 & .000 & .081 & .000 & .000 & .926 \\
\hline & $\mathrm{r}^{2}$ & .329 & 1.000 & .004 & .221 & .457 & .199 & .042 & .221 & .011 & .137 & .051 & .000 \\
\hline & PV & 32.95 & 100 & .43 & 22.09 & 45.70 & 19.89 & 4.24 & 22.09 & 1.06 & 13.69 & 5.06 & .00 \\
\hline \multirow[t]{4}{*}{$\mathrm{Ilb}$} & $r$ & $.038^{\mathrm{S}}$ & $-.066^{\mathrm{S}}$ & 1.000 & $-.131^{*} \mathrm{~S}$ & $-.094^{\mathrm{S}}$ & $.004^{\mathrm{S}}$ & $-.078^{\mathrm{S}}$ & $-.115^{\mathrm{S}}$ & $.381^{* * \mathrm{M}}$ & $-.015^{\mathrm{S}}$ & $-.096^{\mathrm{S}}$ & $-.102^{\mathrm{S}}$ \\
\hline & Sig. & .522 & .266 & & .026 & .110 & .945 & .189 & .051 & .000 & .801 & .106 & .083 \\
\hline & $\mathrm{r}^{2}$ & .001 & .004 & 1.000 & .017 & .009 & .000 & .006 & .013 & .145 & .000 & .009 & .010 \\
\hline & PV & .14 & .43 & 100 & 1.72 & .89 & .00 & .60 & 1.32 & 14.52 & .02 & .91 & 1.05 \\
\hline \multirow[t]{4}{*}{ III } & $r$ & $.565^{+\mathrm{L}}$ & $.470^{*+\mathrm{M}}$ & $-.131^{*} \mathrm{~S}$ & 1.000 & $.557^{* \mathrm{~L}}$ & $.437^{* * \mathrm{M}}$ & $.228^{* *} \mathrm{~S}$ & $.441^{*+\mathrm{M}}$ & $-.020 \mathrm{~s}$ & $.368^{*+\mathrm{M}}$ & $.269^{* * \mathrm{~S}}$ & $.065^{\mathrm{s}}$ \\
\hline & Sig. & .000 & .000 & .026 & & .000 & .000 & .000 & .000 & .735 & .000 & .000 & .269 \\
\hline & $\mathrm{r}^{2}$ & .319 & .221 & .017 & 1.000 & .310 & .191 & .052 & .194 & .000 & .135 & .072 & .004 \\
\hline & PV & 31.92 & 22.09 & 1.72 & 100 & 31.02 & 19.10 & 5.20 & 19.45 & .04 & 13.54 & 7.24 & .43 \\
\hline \multirow[t]{4}{*}{ IV } & $r$ & $.663^{*+\mathrm{L}}$ & $.676^{* \mathrm{~L}}$ & $-.094^{\mathrm{S}}$ & $.557^{* * \mathrm{~L}}$ & 1.000 & $.572^{*+\mathrm{L}}$ & $.248^{* *} \mathrm{~S}$ & $.673^{* \star \mathrm{L}}$ & $-.066^{\mathrm{S}}$ & $.385^{+\mathrm{M}}$ & $.311^{* * \mathrm{M}}$ & $.051^{\mathrm{S}}$ \\
\hline & Sig. & .000 & .000 & .110 & .000 & & .000 & .000 & .000 & .266 & .000 & .000 & .386 \\
\hline & $r^{2}$ & .440 & .457 & .009 & .310 & 1.000 & .327 & .062 & .453 & .004 & .148 & .097 & .003 \\
\hline & PV & 43.96 & 45.70 & .89 & 31.02 & 100 & 32.72 & 6.15 & 45.29 & .43 & 14.82 & 9.67 & .26 \\
\hline \multirow[t]{4}{*}{ V } & $r$ & $.535^{* \star \mathrm{L}}$ & $.446^{* *} \mathrm{M}$ & $.004^{\mathrm{S}}$ & $.437^{* \mathrm{M}}$ & $.572^{* * \mathrm{~L}}$ & 1.000 & $.352^{* * \mathrm{M}}$ & $.512^{* * \mathrm{~L}}$ & $.001^{\mathrm{s}}$ & $.394^{* * M}$ & $.373^{* * \mathrm{M}}$ & $.017^{\mathrm{s}}$ \\
\hline & Sig. & .000 & .000 & .945 & .000 & .000 & & .000 & .000 & .988 & .000 & .000 & .780 \\
\hline & $r^{2}$ & .286 & .199 & .000 & .191 & .327 & 1.000 & .124 & .262 & .000 & .155 & .139 & .000 \\
\hline & PV & 28.62 & 19.89 & .00 & 19.10 & 32.72 & 100.00 & 12.39 & 26.21 & .00 & 15.52 & 13.91 & .03 \\
\hline \multirow[t]{4}{*}{$\mathrm{VI}$} & $\mathrm{r}$ & $.145^{*} \mathrm{~S}$ & $.206^{* * S}$ & $-.078^{\mathrm{S}}$ & $.228^{*+5} \mathrm{~S}$ & $.248^{*+} \mathrm{S}$ & $.352^{* * \mathrm{M}}$ & 1.000 & $.242^{*+5} s$ & $.000^{\mathrm{N}}$ & $.107^{\mathrm{s}}$ & $.205^{*+\mathrm{S}}$ & $-.054^{\mathrm{S}}$ \\
\hline & Sig. & .014 & .000 & .189 & .000 & .000 & .000 & & .000 & .995 & .069 & .000 & .360 \\
\hline & $r^{2}$ & .021 & .042 & .006 & .052 & .062 & .124 & 1.000 & .059 & .000 & .012 & .042 & .003 \\
\hline & PV & 2.10 & 4.24 & .60 & 5.20 & 6.15 & 12.39 & 100.00 & 5.86 & .00 & 1.15 & 4.20 & .29 \\
\hline \multirow[t]{4}{*}{ VIIIa } & $\mathrm{PC}$ & $.456^{*+\mathrm{M}}$ & $.470^{*+\mathrm{M}}$ & $-.115^{\mathrm{s}}$ & $.441^{* \mathrm{M} M}$ & $.673^{*+\mathrm{L}}$ & $.512^{*+\mathrm{L}}$ & $.242^{* * \mathrm{~S}}$ & 1.000 & $-.188^{*+\mathrm{S}}$ & $.382^{++\mathrm{M}}$ & $.326^{* * \mathrm{M}}$ & $.166^{*+\mathrm{S}}$ \\
\hline & Sig. & .000 & .000 & .051 & .000 & .000 & .000 & .000 & & .001 & .000 & .000 & .005 \\
\hline & $r^{2}$ & .208 & .221 & .013 & .194 & .453 & .262 & .059 & 1.000 & .035 & .146 & .106 & .028 \\
\hline & PV & 20.79 & 22.09 & 1.32 & 19.45 & 45.29 & 26.21 & 5.86 & 100.00 & 3.53 & 14.59 & 10.63 & 2.76 \\
\hline \multirow[t]{4}{*}{ VIllb } & $r$ & $.157^{* *} \mathrm{~S}$ & $.103^{\mathrm{s}}$ & $.381^{* \mathrm{M}}$ & $-.020^{\mathrm{s}}$ & $-.066^{\mathrm{s}}$ & $.001^{\mathrm{s}}$ & $.000^{\mathrm{N}}$ & $-188^{* *} \mathrm{~S}$ & 1.000 & $.007^{\mathrm{s}}$ & $-.010^{\mathrm{s}}$ & $-.120^{*} \mathrm{~S}$ \\
\hline & Sig. & .008 & .081 & .000 & .735 & .266 & .988 & .995 & .001 & & .909 & .861 & .042 \\
\hline & $r^{2}$ & .025 & .011 & .145 & .000 & .004 & .000 & .000 & .035 & 1.000 & .000 & .000 & .014 \\
\hline & PV & 2.46 & 1.06 & 14.52 & .04 & .43 & .00 & .00 & 3.53 & 100.00 & .00 & .01 & 1.44 \\
\hline \multirow[t]{4}{*}{ VIIIa } & $r$ & $.374^{* * M}$ & $.370^{* * \mathrm{M}}$ & $-.015^{\mathrm{s}}$ & $.368^{* * \mathrm{M}}$ & $.385^{* * \mathrm{M}}$ & $.394^{* * \mathrm{M}}$ & $.107^{\mathrm{s}}$ & $.382^{*+1 M}$ & $.007^{\mathrm{s}}$ & 1.000 & $.347^{* \times M}$ & $.048^{\mathrm{s}}$ \\
\hline & Sig. & .000 & .000 & .801 & .000 & .000 & .000 & .069 & .000 & .909 & & .000 & .416 \\
\hline & $r^{2}$ & .140 & .137 & .000 & .135 & .148 & .155 & .012 & .146 & .000 & 1.000 & .120 & .002 \\
\hline & PV & 13.99 & 13.69 & .02 & 13.54 & 14.82 & 15.52 & 1.15 & 14.59 & .00 & 100.00 & 12.04 & .23 \\
\hline \multirow[t]{4}{*}{ VIIIb } & $r$ & $.228^{* * S}$ & $.225^{* * S}$ & $-.096^{\mathrm{s}}$ & $.269^{* * \mathrm{~S}}$ & $.311^{* * \mathrm{M}}$ & $.373^{* * \mathrm{M}}$ & $.205^{* *} S$ & $.326^{* * M}$ & $-.010^{\mathrm{S}}$ & $.347^{*+\mathrm{M}}$ & 1.000 & $-.003^{\mathrm{s}}$ \\
\hline & Sig. & .000 & .000 & .106 & .000 & .000 & .000 & .000 & .000 & .861 & .000 & & .964 \\
\hline & $r^{2}$ & .052 & .051 & .009 & .072 & .097 & .139 & .042 & .106 & .000 & .120 & 1.000 & .000 \\
\hline & PV & 5.20 & 5.06 & .91 & 7.24 & 9.67 & 13.91 & 4.20 & 10.63 & .01 & 12.04 & 100.00 & .00 \\
\hline \multirow[t]{4}{*}{ VIIIIC } & $r$ & $.036^{\mathrm{s}}$ & $.005^{\mathrm{s}}$ & $-.102^{\mathrm{S}}$ & $.065^{\mathrm{s}}$ & $.051^{\mathrm{s}}$ & $.017^{\mathrm{s}}$ & $-.054^{\mathrm{S}}$ & $.166^{*+\mathrm{S}}$ & $-.120^{*} \mathrm{~S}$ & $.048^{\mathrm{S}}$ & $-.003^{\mathrm{S}}$ & 1.000 \\
\hline & Sig. & .544 & .926 & .083 & .269 & .386 & .780 & .360 & .005 & .042 & .416 & .964 & \\
\hline & $\mathrm{r}^{2}$ & .001 & .000 & .010 & .004 & .003 & .000 & .003 & .028 & .014 & .002 & .000 & 1.000 \\
\hline & PV & 0.13 & 0.00 & 1.05 & 0.43 & 0.26 & 0.03 & 0.29 & 2.76 & 1.44 & 0.23 & 0.00 & 100.00 \\
\hline
\end{tabular}

$\mathbf{r}=$ Pearson Correlation; Sig. = Sig. (2-tailed); $\mathbf{r}^{2}=$ r squared; $\mathbf{P V}=$ percent shared variance

**. Correlation is significant at the 0.01 level (2-tailed);

*. Correlation is significant at the 0.05 level (2-tailed); a. Listwise $\mathrm{N}=288$

$\mathrm{L}=$ large; $\mathrm{M}=$ medium; $\mathrm{S}=$ small; $\mathrm{N}=$ no linear correlation

Table 31. The number of large, medium, and small correlations for each dimension

\begin{tabular}{|c|c|c|c|c|}
\hline Scales & $\begin{array}{c}\text { Large } \\
(\mathrm{r}= \pm .50 \text { to } \pm \mathbf{1 . 0})\end{array}$ & $\begin{array}{c}\text { Medium } \\
(\mathrm{r}= \pm .30 \text { to } \pm .49)\end{array}$ & $\begin{array}{c}\text { Small } \\
(\mathrm{r}= \pm .10 \text { to } \pm .29)\end{array}$ & $\begin{array}{c}\text { No linear } \\
\text { correlation/ } \\
\text { uncorrelated }\end{array}$ \\
\hline 1. TOTALI I & 4 & 2 & 5 & - \\
\hline 2. TOTAL III & 2 & 4 & 5 & - \\
\hline 3. TOTAL Ilb & - & 1 & 10 & - \\
\hline 4. TOTAL III & 2 & 4 & 5 & - \\
\hline 5. TOTAL IV & 5 & 2 & 4 & - \\
\hline 6. TOTAL V & 3 & 5 & 3 & - \\
\hline 7. TOTAL VI & - & 1 & 9 & - \\
\hline 8. TOTAL VIIIa & 2 & 5 & 4 & - \\
\hline 9. TOTAL VIIb & - & 1 & 9 & - \\
\hline 10. TOTAL VIIIla & - & 7 & 4 & - \\
\hline 11. TOTAL VIIIb & - & 4 & 7 & \\
\hline 12. TOTAL VIIIC & - & - & 11 & \\
\hline
\end{tabular}




\subsection{Step Three: Dimension readiness evaluation}

This section presents the third step of the quantitative data analysis. It evaluates each dimension of readiness at FORDA. This section also addresses the level of organisational readiness for OES in each dimension, and identifies critical factors that need to be considered in order to implement successful OES at FORDA.

Table 32 presents the percentages of organisational readiness for implementing OES at FORDA. The level of readiness in each dimension was assessed individually. Each percentage of readiness was calculated by using this formula as presented in Table 32, Column F:

$$
\text { Percentage of readiness }=\frac{\text { the mean score of dimension } n}{\text { the maximum scale range of dimension } n} \times 100
$$

\begin{tabular}{|c|c|c|c|c|c|}
\hline \multirow{2}{*}{$\begin{array}{c}\text { DVs } \\
\text { (scale) }\end{array}$} & \multirow{2}{*}{ Dimensions } & \multirow{2}{*}{ Mean } & \multicolumn{2}{|c|}{ Scale range } & \multirow{2}{*}{$\begin{array}{c}\% \text { of } \\
\text { readiness } \\
((\mathrm{C} / \mathrm{E}) \times 100))\end{array}$} \\
\hline & & & Min & Max & \\
\hline$A$ & B & C & D & E & $\mathbf{F}$ \\
\hline TOTAL I & Technological skills readiness & 19.6875 & 6 & 30 & 65.63 \\
\hline TOTAL IIa & Positive online learning style readiness & 19.0104 & 6 & 30 & 63.36 \\
\hline TOTAL IIb & Negative online learning style readiness & 6.8785 & 3 & 15 & $45.86^{*}$ \\
\hline TOTAL III & Equipment/infrastructure readiness & 31.3715 & 8 & 40 & 78.43 \\
\hline TOTAL IV & Attitude readiness & 89.5347 & 23 & 115 & 77.86 \\
\hline TOTAL V & Human resource readiness & 29.3611 & 11 & 55 & 53.38 \\
\hline TOTAL VI & Environmental readiness & 13.3924 & 4 & 20 & 66.96 \\
\hline TOTAL VIIa & Positive cultural readiness & 15.7083 & 4 & 20 & 78.54 \\
\hline TOTAL VIIb & Negative cultural readiness & 4.7188 & 2 & 10 & 47.19 ** \\
\hline TOTAL VIIIa & Financial affordability readiness & 7.2118 & 3 & 15 & 48.08 \\
\hline TOTAL VIIIb & Financial availability readiness & 6.7813 & 2 & 10 & 67.81 \\
\hline TOTAL VIIIC & Financial possibility readiness & 6.5903 & 2 & 10 & $65.90 / 34.1^{\text {*t+ }}$ \\
\hline
\end{tabular}

Table 32. The level of readiness at FORDA

* The percent of readiness on negative online learning style was $45.86 \%$. This scale explains conventional learning rather than e-learning implying that FORDA has less preference for conventional learning style.

** The percent of readiness on negative cultural was $47.19 \%$. This scale explains conventional learning rather than e-learning implying that FORDA has less preference for conventional learning culture.

*** The initial result of readiness on financial possibility was $65.90 \%$. Nevertheless, it is critical to note that in fact the type of two items (CVIII6 and CVIII7) within the scale were negative worded question. Nonetheless, they were not reversed during the analysis. This implies that the result should be read on the other way around. In other word, the level of readiness on financial possibility was actually the lowest at $34.1 \%$.

The assessment of readiness in this study was developed based on an process used by Aydin and Tasci (2005). The COERI used a five-point Likert scale in which each answer was coded into 1, 2, 3, 4, and 5 (Figure 15 Line A); therefore the critical level 
was 0.8 (4 intervals divided by 5 categories). Aydin and Tasci (2005, p. 250) added this critical level iteratively from the lowest category "1" until reaching the highest point "5"; accordingly five intervals of readiness were obtained and they considered 3.4 as the expected level of readiness (Figure 15 line B). In this study however, the level of readiness was calculated using a percentage of readiness as calculated in Table 32. Therefore, the levels of readiness were determined as presented in Figure 15 Line C. In the next few sections, the level of readiness of each dimension will be described.

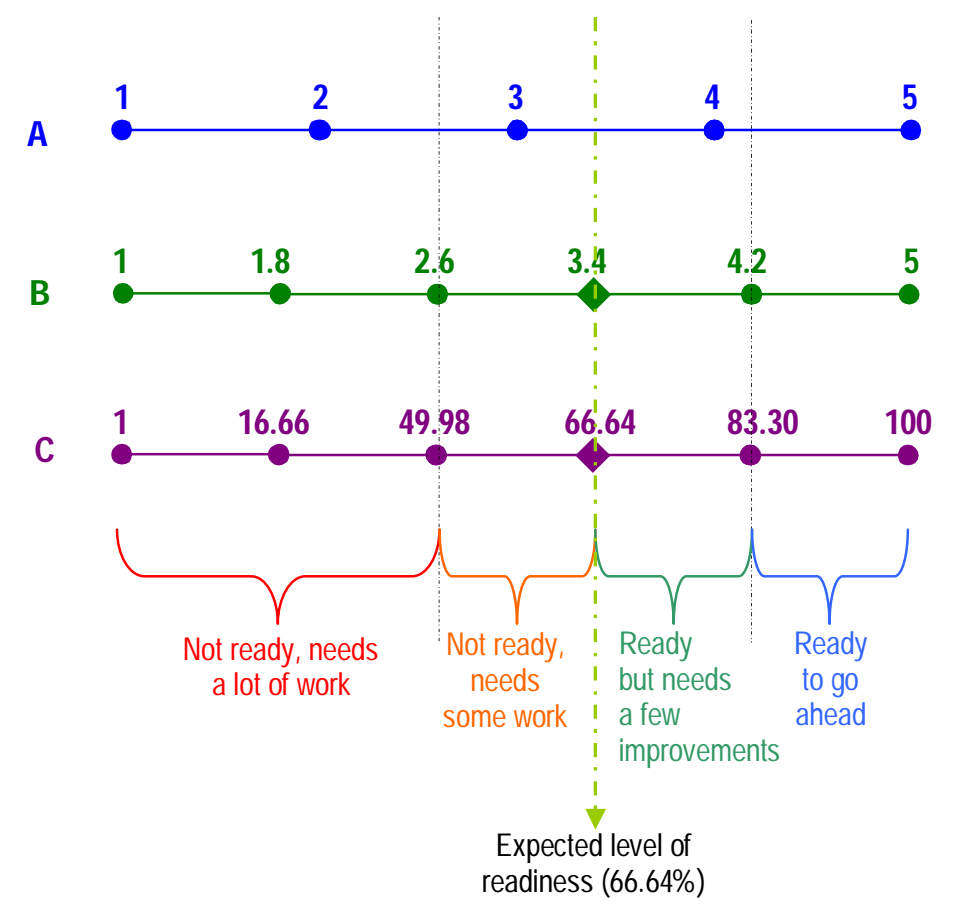

Figure 15: Assessment readiness of COERI

(Adapted from Figure 1, Aydin \& Tasci, 2005, p. 250)

\subsubsection{TOTAL I - technological skills readiness}

Technological skills readiness refers to observable and measurable technical competencies. Previous studies indicate that this dimension is very important for adopting e-learning (Abas, Kaur, \& Karun, 2004; Aydin \& Tasci, 2005; Chapnick, 2000; EIU \& IBM, 2003; Pillay et al., 2007; Sadik, 2007; Watkins et al., 2004). However, the score of readiness in technological skills was only $65.63 \%$; thus it is considered not ready and needs some work to improve it.

Figure 16 presents the summary of technological skills readiness. More than half of the respondents $(68.4 \%)$ had the basic skills to operate a computer. Less than half of the respondents (47.3\%) were good at using basic software packages. A third of the respondents $(33 \%)$ could install software on a computer to support their learning. Less 
than half of the respondents $(43.3 \%)$ were familiar with Internet skills (browsing, searching materials, entering passwords, etc). Slightly over half of the respondents $(58.4 \%)$ could use basic email. In addition, only $10.4 \%$ of respondents could troubleshoot most problems associated with using a computer. Age is the strongest and a statistically significant predictor ( $\beta=-.479 ; \mathrm{p}=.000)$ of TOTAL I, followed by education $(\beta=.258 ; \mathrm{p}=.000)$, B2athome $(\beta=-.209 ; \mathrm{p}=.000)$, Blatwork $(\beta=-.208 ; \mathrm{p}=.000)$, and gender $(\beta=.208 ; \mathrm{p}=.000)$. Older respondents have lower scores in technological skills readiness. Those with a higher level of education also have a higher level of technological skills. Respondents who have an Internet connection at home and at work have a higher level of technological skills readiness. Males are more ready than females in this scale. In this regard, these results suggest that older employees, employees with lower level qualifications, and females could be targeted for training to improve technological skills readiness. In addition, the provision of Internet access is also important to improve their readiness.

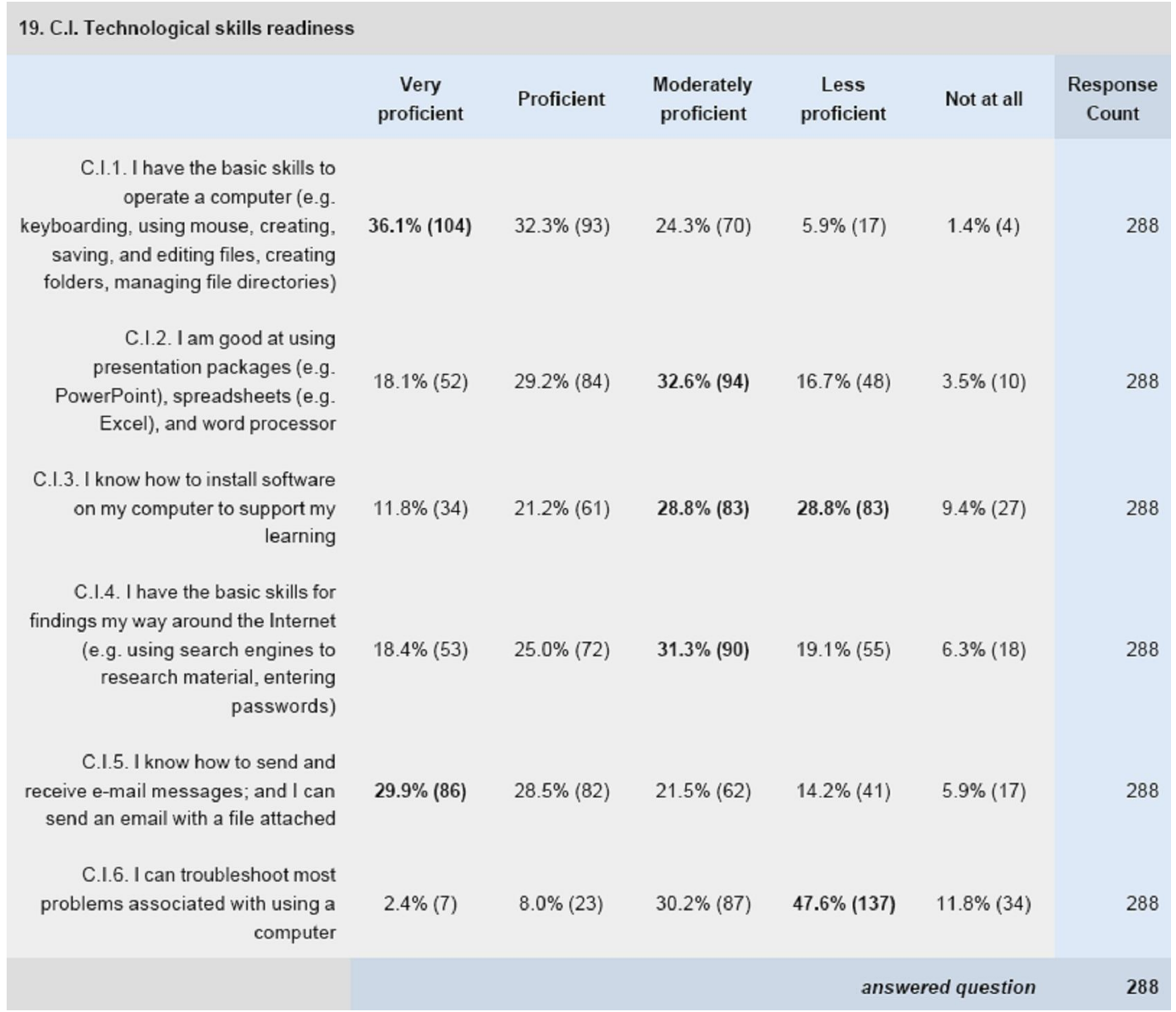

Figure 16: Summary of technological skills readiness 


\subsubsection{TOTAL Ila - positive online learning style readiness}

Positive online learning style readiness involves learners' readiness in terms of time commitment to e-learning, discipline and interest in e-learning, and perception of the status of qualifications obtained via e-learning. Previous studies considered this dimension to be important (Pillay et al., 2007; Smith, 2005; Watkins et al., 2004). The results show that the level of positive online learning style readiness at FORDA was not ready at $63.36 \%$, and needs some work to improve its readiness. Figure 17 presents the summary of online learning style readiness.

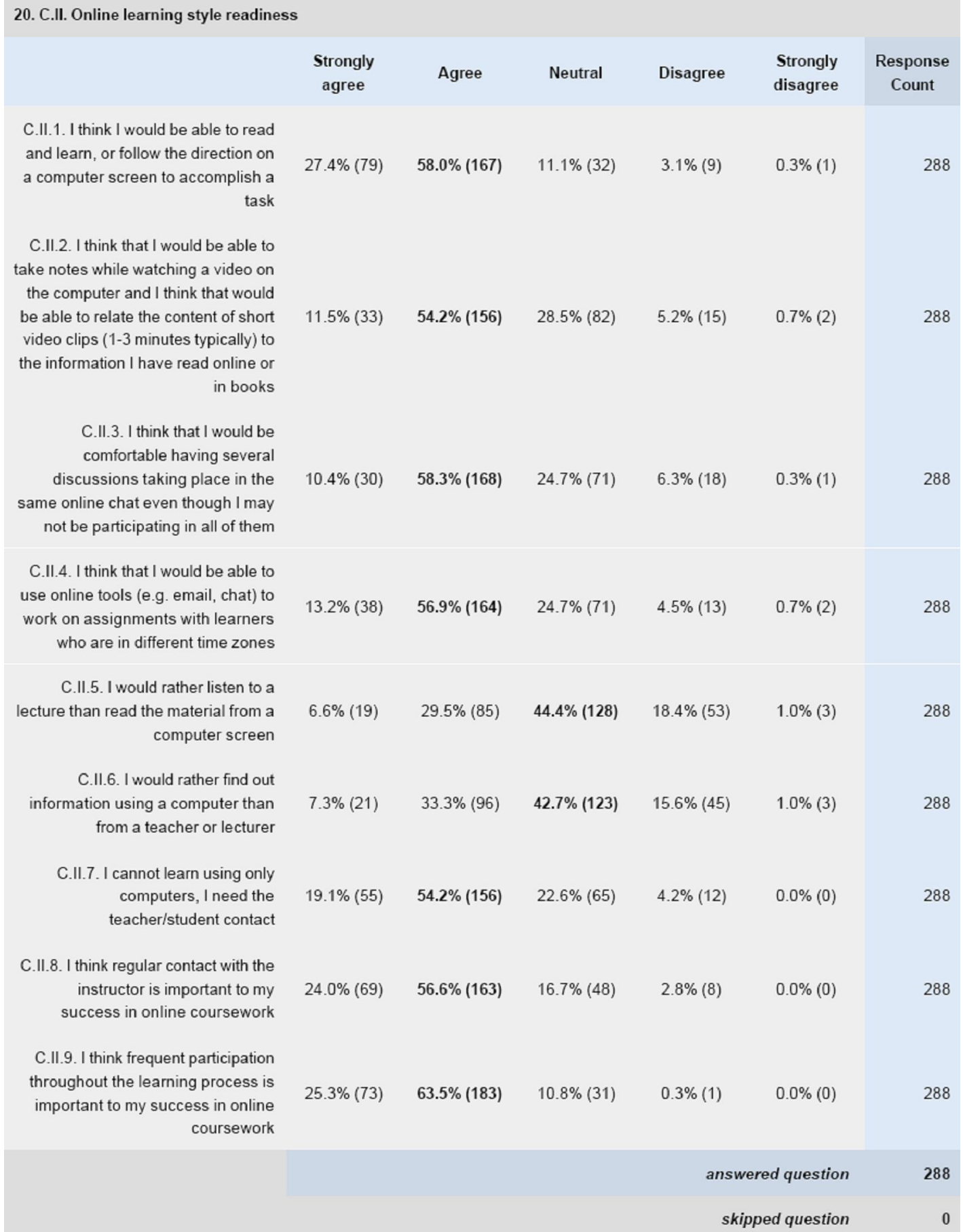

\section{Strongly}

agree

Agree

Neutral

Disagree

Strongly

disagree

Response

Count

$27.4 \%(79) \quad 58.0 \%(167) \quad 11.1 \%(32) \quad 3.1 \%(9) \quad 0.3 \%(1)$

$28.5 \%(82)$

$5.2 \%(15)$

$0.7 \%(2)$

$10.4 \%(30) \quad 58.3 \%(168) \quad 24.7 \%(71) \quad 6.3 \%(18) \quad 0.3 \%(1) \quad 288$

$13.2 \%(38) \quad 56.9 \%(164) \quad 24.7 \%(71) \quad 4.5 \%(13) \quad 0.7 \%(2) \quad 288$

$6.6 \%(19) \quad 29.5 \%(85) \quad 44.4 \%(128) \quad 18.4 \%(53) \quad 1.0 \%(3) \quad 288$

$\begin{array}{llllll}7.3 \%(21) & 33.3 \%(96) & 42.7 \%(123) & 15.6 \%(45) & 1.0 \%(3) & 288\end{array}$

$19.1 \%(55) \quad 54.2 \%(156) \quad 22.6 \%(65) \quad 4.2 \%(12) \quad 0.0 \%(0) \quad 288$

$24.0 \%(69) \quad 56.6 \%(163) \quad 16.7 \%(48) \quad 2.8 \%(8) \quad 0.0 \%(0) \quad 288$

$25.3 \%(73) \quad 63.5 \%(183) \quad 10.8 \%(31) \quad 0.3 \%(1) \quad 0.0 \%(0) \quad 288$

answered question 
Most (73.3\%) of the respondents believed that they cannot learn using computers only; they needed contact with other students or facilitators. Only $19.4 \%$ of the respondents preferred to read material from a computer screen rather than listen to a lecture. Additionally, only $40.6 \%$ of respondents agreed that they would rather find out information using a computer than from a teacher or lecturer. Many (80.6\%) of the respondents perceived regular contact with instructors as important to their success in online coursework. These indicate that to some extent the online learning style at FORDA was not ready and requires some effort to improve it.

Age $(\beta=-.351 ; \mathrm{p}=.000)$ is the strongest and a significant predictor of TOTAL IIa, followed by B2athome ( $\beta=-.212 ; \mathrm{p}=.000)$, and education $(\beta=.188 ; \mathrm{p}=.009)$. These indicate that older respondents have lower scores on the positive online learning readiness scale; respondents who have an Internet connection at home have a higher level of readiness; and those with higher level of education have higher scores. Thus, to improve positive online learning style readiness at FORDA, it is important to target older employees and employees with lower level qualifications. Providing Internet access is also important to improve the online learning style.

\subsubsection{TOTAL Ilb - negative online learning style readiness}

Negative online learning style readiness refers to the lack of learner readiness in terms of time commitment to e-learning, discipline and interest in e-learning, and perception of the status of qualifications obtained via e-learning. FORDA had a low percentage of readiness on negative online learning style (45.86\%). However, this scale explains conventional learning readiness rather than e-learning. Thus this implies that almost half of the respondents have a preference for conventional learning styles rather than online learning. In this case, the online learning style needs to be improved considerably which confirms the results from TOTAL IIa.

Additionally, none of the IVs make a significant contribution to TOTAL IIb. All IVs have low $ß$ values with $\mathrm{p}$ values greater than 0.05 . This indicates that education, gender, age, Internet access, and job title do not have a significant influence on negative online learning style. 


\subsubsection{TOTAL III - infrastructure/equipment readiness}

Infrastructure/equipment readiness refers to the provision of technical support, elearning content delivery, broadband facilities, and a Learning Management System (LMS) by the organisations who adopt the systems. Connectivity and physical communications infrastructure are the foundation of electronic-readiness for a country (EIU \& IBM, 2008). Although Indonesia had very low scores of e-readiness in connectivity at about 2.3 out of 20 (EIU \& IBM, 2008), the results of this study show that more than half of the respondents $(55.3 \%)$ had used Internet technology to perform daily operations at FORDA. Most (86.8\%) of the respondents had Internet access at work, but only $19.4 \%$ had Internet access at home. Infrastructurelequipment readiness ranked second at FORDA (78.43\%). Thus, it is considered ready but still needs a few improvements. Blatwork $(\beta=-.321 ; \mathrm{p}=.000)$ is the strongest significant predictor of TOTAL III, followed by age ( $(=-.290 ; \mathrm{p}=.000)$, education $(\beta=.261 ; \mathrm{p}=.000)$, and gender $(\beta=.109 ; p=.043)$. These indicate that respondents who have an Internet connection at work have higher scores in equipment/infrastructure readiness; older respondents have lower scores; respondents with a higher level of education have higher scores; and males have higher scores than females. In this regard, to maintain equipment/infrastructure readiness it is vital to improve Internet access at work. Additionally, training should target older employees, and employees with lower education levels.

\subsubsection{TOTAL IV - attitude readiness}

Attitude readiness involves confidence, enjoyment, importance, motivation, selfdevelopment, and anxiety. Attitude readiness ranked third at FORDA (77.86\%), indicating that it is ready but a few improvements are required. Previous studies noted that attitude directly affects individual readiness for e-learning (Aydin \& Tasci, 2005; Chapnick, 2000; Pillay et al., 2007; Sadik, 2007; Smith, 2005; Watkins et al., 2004). Age $(\beta=-.349 ; \mathrm{p}=.000)$ is the strongest and a significant predictor of TOTAL IV, followed by education $(\beta=.244 ; p=.001)$, gender $(\beta=.161 ; p=.003)$, B2athome $(\beta=-.137$; $\mathrm{p}=.011)$, and Blatwork $(\beta=-.125 ; \mathrm{p}=.021)$. Older people have lower scores in their attitude readiness. Respondents who have higher education levels have higher scores. Males are more ready than females. Respondents who have an Internet connection at home and at work have higher levels of attitude. Thus, to uphold attitude readiness, it is 
important to deliver training to older employees, employees with lower levels of qualifications, and females. It is also important to improve Internet access.

\subsubsection{TOTAL V - human resource readiness}

Human resources readiness is the availability and design of the human support system including management (having a vision/mission or formulated policies related to the provision of e-learning and the organisational recognition of qualifications obtained via e-learning) and personnel (having a central unit dedicated to e-learning initiatives with a team of dedicated instructional designers and a staff development plan for e-learning). This dimension is vital (Abas, Kaur, \& Karun, 2004; Aydin \& Tasci, 2005; Chapnick, 2000; Sadik, 2007). Yet, the score of human resource readiness at FORDA was low $(53.08 \%)$, indicating that human resources were not ready and some work is needed to improve their readiness. Education $(\beta=.220 ; \mathrm{p}=.004)$ is the strongest and a significant predictor of TOTAL V, followed by Blatwork $(\beta=-.202 ; p=.001)$, and gender $(\beta=.160$; $\mathrm{p}=.007)$. These indicate that a higher level of education corresponds to a higher level of readiness; respondents who have an Internet connection at work have higher scores; and males are more ready than females. Figure 18 provides the summary of human resource readiness.

Although more than half the respondents believed that their upper level management was ready for supporting and engaging themselves in e-learning, the levels of vital proficiency or abilities to organise and facilitate OES were considerably low. Only about $11.8 \%$ of respondents were experienced in organising and evaluating training and able to help other employees with career development. About 13.2\% rof espondents were able to facilitate the acceptance and implementation of e-learning initiatives at FORDA. Similarly, only $13.2 \%$ of respondents were familiar with computer-based training or multimedia-based learning. Respondents who believed that they could design web-pages for e-learning comprised only $2.7 \%$. Only $4.9 \%$ of respondents believed that they could moderate online discussions. Similarly, only $4.2 \%$ of respondents thought that they could write effective study guides for e-learning purposes. Finally, only $1.7 \%$ of respondents were able to deal with legal issues related to e-learning (e.g. copyright and privacy). 


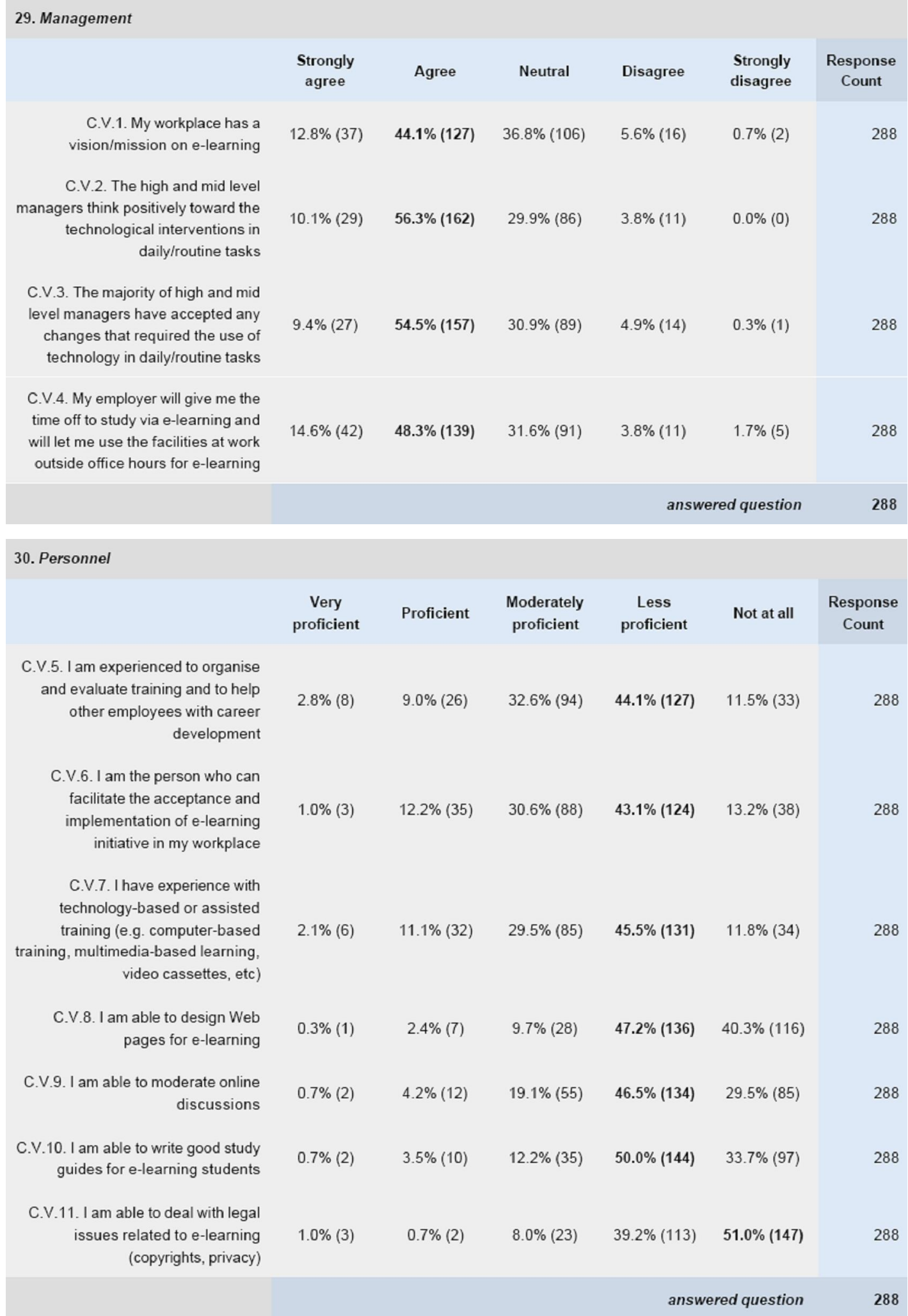

Figure 18: Summary of human resource readiness

In view of these, it is clear that training is vital to improve personnel skills in order to successfully implement OES at FORDA. Education, Blatwork, and gender are significant predictors of human resource readiness. Employees with a higher level of education and males scored higher on this scale suggesting that employees with lower 
levels of qualifications and females should be targeted for training. Moreover, those who have Internet access have higher scores of readiness suggesting the necessity to develop Internet access.

\subsubsection{TOTAL VI - environmental readiness}

Environmental readiness refers to the level of readiness of a society/nation for elearning as perceived by stakeholders (policy makers, providers, enablers, and learners/trainees) from within and outside the organisation, and involves the readiness of the organisation as a whole in terms of government policy, the role of mass media, and intellectual property regulations. According to EIU and IBM (2008), Indonesia had very low scores of electronic readiness (e-readiness) in its social and cultural environment (3.53 out of 15), legal environment (3.20 out of 10), and government policy and vision (3.40 out of 15). Yet, this research reveals that the level of environmental readiness at FORDA was overall ready at $66.96 \%$ but needs a few improvements. None of the IVs (age, gender, education, Blatwork, and B2athome) are important for environmental readiness and they explain a very low percentage of variance on environmental readiness.

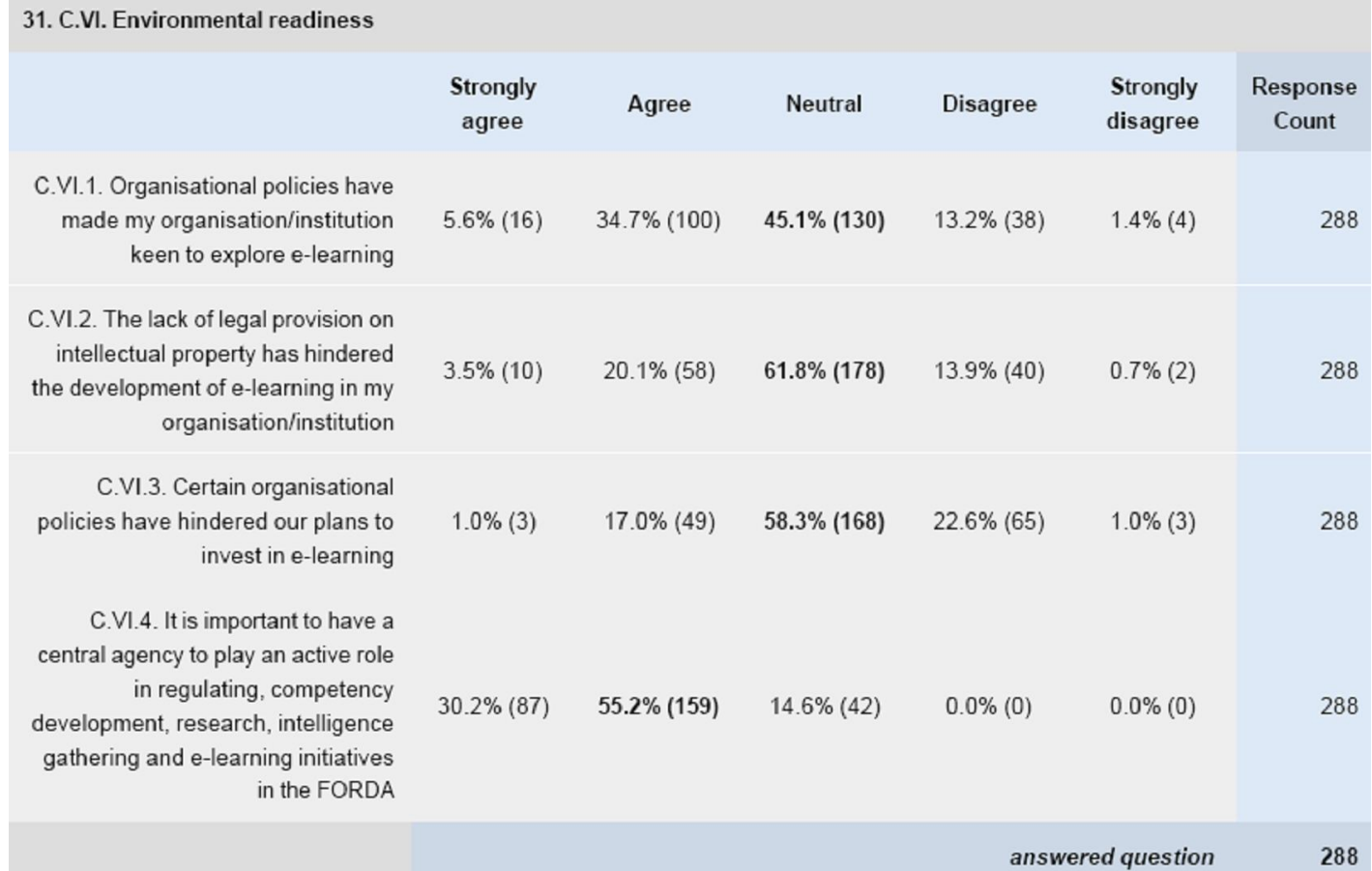

Figure 19: Summary of environmental readiness 
Figure 19 presents the summary of environmental readiness. Some $(40.3 \%)$ respondents believed that organisational policies have made the organisation keen to explore elearning. Most (85.4\%) respondents agreed that it is vital to have a central e-learning agency at FORDA. Almost a quarter (23.6\%) of respondents believed that the lack of legal provision on intellectual property has hindered e-learning development and implementation at FORDA. A smaller proportion (18\%) of respondents agreed that certain organisation policies have hindered their plans to invest in e-learning.

The results suggest that a centralised e-learning group which is sufficiently supported by the organisation is required. Additionally, the legal provisions for the protection of intellectual property were not yet available. Intellectual property regulations also need to be formulated and disseminated to the whole organisation before implementing the systems. There is a need to guide employees and to make sure that they know how to cite and to choose and use valid information gained from the online environment. A similar situation was revealed by Abas, Kaur, and Karun (2004) who measured elearning readiness in the developing country context of Malaysia. However, it is necessary to further explore the environmental readiness of FORDA as the scale has a very poor reliability result.

\subsubsection{TOTAL VIla - positive cultural readiness}

Positive cultural readiness refers to stakeholders' (policy makers, enablers, and learners/trainees) acceptance of Internet use and network technologies as a mode for information dissemination, communication, interaction, and teaching; and the readiness for making e-learning a way of life in their organisation. Positive culture towards OES significantly affects OES implementation (Ali \& Magalhaes, 2008; Aydin \& Tasci, 2005; Condie \& Livingston, 2007; Nichols, 2007; Sadik, 2007; Stokes et al., 2004; White, 2007). The area most prepared to implement OES at FORDA was positive cultural readiness at $78.54 \%$, indicating that the online culture at FORDA is overall ready but a few improvements are needed. Education $(\beta=.259 ; \mathrm{p}=.001)$ is the strongest and a significant predictor of TOTAL VIIa, followed by Blatwork ( $\beta=-.246 ; p=.000)$. These indicate that respondents who have higher levels of education have higher levels of positive cultural readiness; and respondents who have an Internet connection at work have higher level of readiness for online culture. In this regard, to maintain positive cultural readiness training should be delivered to employees with low levels of 
qualifications. Providing Internet access at work is also vital to enhance their positive cultural readiness.

\subsubsection{TOTAL VIIb - negative cultural readiness}

Negative cultural readiness is the inverse of positive online culture readiness which means that it measures the conventional culture rather than online culture. This dimension had a low percentage of readiness at $47.19 \%$. As this scale explains conventional culture rather than online culture readiness thus it implies that almost half of the respondents have a preference for conventional culture rather than online culture. In this regard, the online culture needs to be improved considerably which to some extent confirms the results from TOTAL VIIa about the improvements needed. Therefore, it means that positive online culture was not the most prepared area at FORDA.

Moreover, none of the IVs make a significant contribution to TOTAL VIIb. All IVs have considerably low $\beta$ values with $p$ values greater than 0.05 . This indicates that education, gender, age, Internet access, and job title do not have a significant influence on negative cultural readiness.

\subsubsection{TOTAL VIIla - financial affordability}

Financial affordability readiness refers to the ability to afford the required equipment and facilities for OES implementation as perceived by policy makers, enablers, and learners/trainees. The level of readiness on financial affordability was a low $48.08 \%$ indicating that it was not ready and a lot of effort is needed to improve its readiness. Blatwork $(\beta=-.156 ; \mathrm{p}=.010)$ is the strongest and a significant predictor of TOTAL VIIIa, followed by B2athome ( $\beta=-.144 ; \mathrm{p}=.016)$. These indicate that respondents who have an Internet connection at work or at home have higher levels of readiness in this scale. The summary of financial affordability is presented in Figure 20 items CVIII1, CVIII2, and CVIII3. Only $11.8 \%$ of respondents were confident that FORDA would provide a computer loan to employees. A quarter (24.7\%) of respondents were confident to take out a loan to buy a computer to support their learning. Slightly fewer $(23.3 \%)$ respondents could afford to buy a computer and pay for Internet access. These imply that to improve e-learning readiness, it is important to provide a computer loan for employees and to provide them with Internet access. 


\subsubsection{TOTAL VIIIb - financial availability}

Financial availability readiness refers to the availability of funds to develop and/or acquire OES in the organisation as perceived by policy makers, enablers, and learners/trainees. The percentage of financial availability readiness was $67.81 \%$, indicating that it is ready but needs a few improvements. The summary of financial availability is presented in Figure 20 items CVIII4 and CVIII5. Many (69.1\%) respondents believed that FORDA can create a budget for implementing OES. However, less than a third (31.6\%) of respondents were confident that FORDA provides sufficient funds for employees to attend training and conferences. This implies that to successfully implement OES at FORDA, it is important to allocate more funds for training and conferences. None of the IVs make a significant contribution to TOTAL VIIIb. All IVs have considerably low $B$ values with $\mathrm{p}$ values greater than 0.05 indicating that education, gender, age, Internet access, and job title do not have a significant influence on financial availability readiness.

\subsubsection{TOTAL VIIIc - financial possibility}

Financial possibility readiness refers to the possibility of allocating funds to develop and/or acquire OES in the organisation as perceived by policy makers, enablers, and learners/trainees. Of the three financial readiness subscales, financial possibility had the lowest level of readiness at $34.1 \%$, indicating that it was not ready and needs a lot of work to improve its readiness. The summary of financial possibility is presented in Figure 20 items CVIII6 and CVIII7. Only $21.5 \%$ of respondents did not think that high initial investment may hinder the organisation in developing and implementing elearning. Moreover, only $15.2 \%$ of respondents did not think that high operating costs may hinder the organisation from developing and implementing e-learning. Gender ( $\beta=-$ $.157 ; \mathrm{p}=.012)$ is the strongest and a significant predictor of TOTAL VIIIc, indicating that the level of financial possibility readiness is significantly predicted by gender as females are more ready than males. 


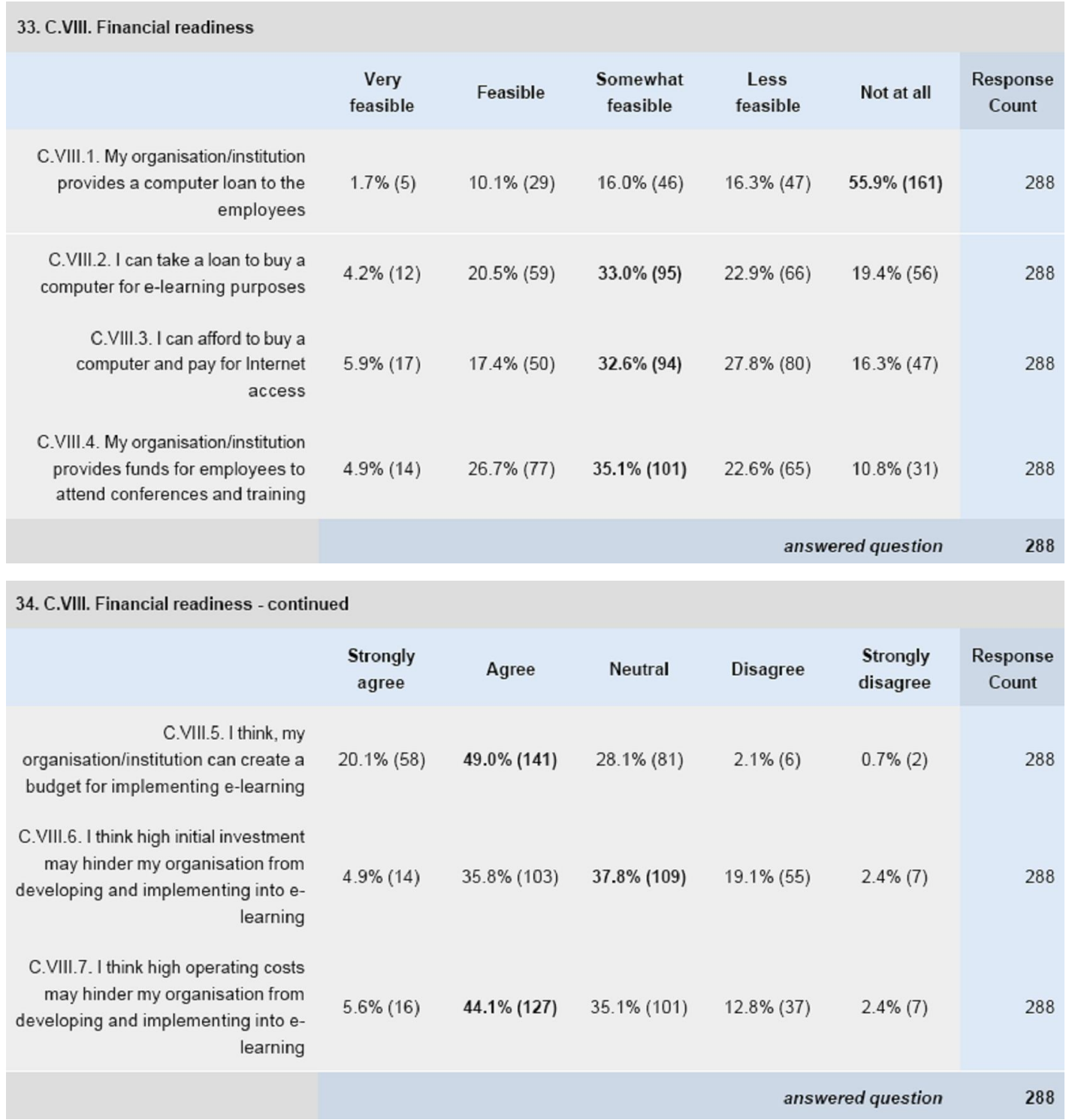

Figure 20: Summary of financial readiness

\subsection{Summary}

Table 33 presents the summary of the strongest predictors of the scales. Firstly, it is important to note that TOTALs IIb and VIIb are scales that measure conventional learning. Additionally, these two scales and TOTAL VIIIc consisted of negatively worded questions. Therefore the results of readiness in these three scales demonstrated a different direction from the rest of the scales. 
Table 33. The strongest predictors

\begin{tabular}{|c|c|c|c|}
\hline \multirow{2}{*}{ IVs } & \multicolumn{3}{|c|}{ STRONGEST CONTRIBUTION TO } \\
\hline & DVs & Scale & Significant \\
\hline \multirow[t]{3}{*}{ Age } & TOTAL I & Technological skills & YES \\
\hline & TOTAL \|la & Positive online learning style & YES \\
\hline & TOTAL IV & Attitude & YES \\
\hline Gender & TOTAL VIIIC & Financial possibility & YES \\
\hline \multirow[t]{4}{*}{ Education } & TOTAL V & Human resource & YES \\
\hline & TOTAL VI & Environmental & NO \\
\hline & TOTAL VIla & Positive cultural readiness & YES \\
\hline & TOTAL VIIIIb & Financial availability & NO \\
\hline Job title & - & - & - \\
\hline \multirow[t]{3}{*}{ B1atwork } & TOTAL Ilb & Negative online learning style & NO \\
\hline & TOTAL III & Equipment/infrastructure & YES \\
\hline & TOTAL VIIIIa & Financial affordability & YES \\
\hline B2athome & TOTAL VIIb & Negative cultural readiness & NO \\
\hline
\end{tabular}

Age is the strongest and a significant predictor of TOTALs I, IIa, and IV. Ten scales have negative relationships indicating that younger respondents have higher scores in the scales. This shows that younger people are more ready for OES, whilst older people tend to be less ready to adopt OES. This result verifies a previous study by Pillay et al. (2007) confirming that older students had lower technical skills and computer selfefficacy than younger students.

Education is the strongest and a significant predictor of TOTALs V and VIIa. It is also the strongest but not a significant predictor of TOTALs VI and VIIIb. Eleven scales have positive relationships indicating that respondents who have higher levels of education have higher levels of readiness. Only one scale, TOTAL IIb, has a negative sign indicating that those with higher levels of education have lower scores in this scale. Blatwork is the strongest and a significant predictor of TOTALs III and VIIIa. It is also the strongest but not a significant predictor of TOTAL IIb. Eleven scales have negative relationships indicating that respondents who have an Internet connection at work have higher scores in the scale. Only one scale, TOTAL VIIb, has a positive relationship indicating that respondents who have an Internet connection at work have lower scores in this scale.

B2athome is the strongest but not a significant predictor of TOTAL VIIb. Eight scales have negative relationships indicating that respondents who have Internet access at home have higher scores in the scale. Four scales have positive relationships (TOTALs IIb, VI, VIIIb, and VIIIc), indicating that respondents who have Internet access at home have lower scores in these scales. 
Gender has the strongest and a significant contribution only for TOTAL VIIIc. Four scales have negative relationships (TOTALs VIIb, VIIIa, VIIIb, and VIIIc), indicating that females have higher scores than males in these four scales. On the other hand, the other eight scales show that males have higher scores. Although gender is the strongest predictor, it is not clear what this means. Further exploration might be needed to specifically assess OES readiness between genders.

When age, gender, education and Internet access are accounted for, job title was not a significant predictor in any of the twelve scales. Job title had low $\beta$ values and the $p$ values were greater than 0.05 indicating that there were no statistically significant differences among the different types of employees when their age, gender, education and Internet access are adjusted for.

To sum up, age is significant for technological, positive online learning style and attitude readiness. Education is also significant for human resource and positive cultural readiness. Internet access at work is significant for equipment/infrastructure readiness and financial affordability readiness; whilst Internet access at home is not a significant predictor in any of the scales. Gender is significant for financial possibility: females have higher scores than males. Further research may be needed to investigate this finding. Overall, these findings contradicted previous findings by Aydin and Tasci (2005) which confirmed that gender, age, education level, and computer experience have no effect on the overall perception of organisational readiness.

The results show that overall five areas are ready at FORDA but need a few improvements: equipment/infrastructure, attitude, environment, positive online culture, and financial availability. Three areas are not ready and need some work to improve their readiness: technological skills, positive online learning style, and human resources. The areas that are not ready and still need a lot of work for improvement are: financial affordability, and financial possibility. As discussed above, two dimensions, negative online learning style and negative culture explain conventional learning style and conventional culture rather than online learning style and online culture. The percentages of readiness in these two areas were nearly 50\%, implying that the level of readiness in online learning style and online culture still need to be improved. Figure 21 presents the percentage of organisational readiness for OES at FORDA. 


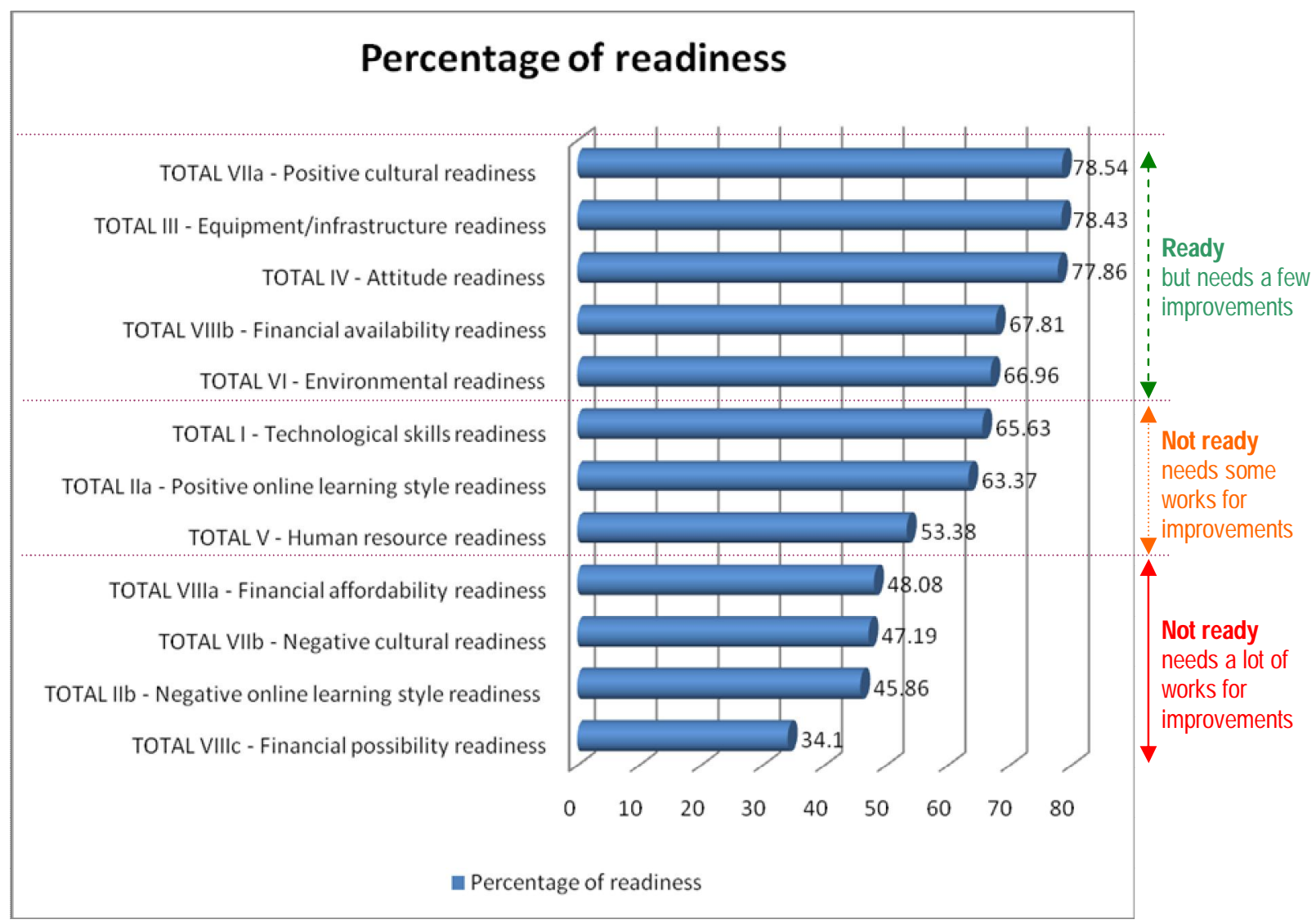

Figure 21: The level of readiness at FORDA 


\section{Chapter 5: Phase II - Exploratory (Semi-structured interviews)}

The main purpose of this second phase is to support the first phase of the research by gaining a more depth exploration of the phenomenon being researched. It attempts to gather important information that might have been missed out from the quantitative data collection in order to address the research questions. The first goal of this phase was to investigate the level of organisational readiness to implement OES at FORDA. Moreover, the second goal was to determine critical factors that need to be considered in order to implement successful OES at FORDA. This chapter describes the second phase of the research. It provides a detailed explanation of the qualitative data analysis which was conducted in three main steps:

\section{Step ONE: Semi-structured interview procedure development}

This step comprised the preparation for the qualitative data collection for this research.

\section{Step TWO: Recognising the level of organisational readiness for OES}

This step involved the investigation of organisational readiness for OES in each regional office. It addressed the first research question: the level of organisational readiness to implement OES at FORDA.

\section{Step THREE: Identifying potential barriers to OES implementation}

Step three entailed the examination of factors or barriers that may occur in each regional office which would hinder the implementation of OES. It addressed the second research question: the critical factors that need to be considered in order to implement successful OES at FORDA.

This chapter is organised as follows:

5.1 Step One: Semi-structured interview development

5.2 Step Two: The level of organisational readiness for OES

5.3 Step Three: Barriers to OES implementation

\subsection{Summary}




\subsection{Step One: Semi-structured interview development}

The semi-structured interviews were designed to meet the goals of this phase involving the literature review on assessing organisational readiness for OES implementation. Other constructs revealed by previous studies were used to set up the questions. The interview questions were mostly taken from a study by Ali and Magalhaes (2008). However, the structure was customised to a research context with no prior e-learning knowledge. Appendix 13 shows the interview questions.

The goals of the semi-structured interviews were to assist the investigation and the explanation of the level of organisational readiness for OES and the identification of critical factors that need to be considered in order to implement OES particularly at a research context with no prior e-learning knowledge. Thus, five questions were developed to gain deeper insights into these issues. The questions were particularly set up to gather information about:

(1) the possibility of OES implementation and the awareness of OES at FORDA;

(2) the availability of support such as incentives to encourage people at FORDA to be involved in OES;

(3) the existence of potential challenges in setting up and implementing OES at FORDA;

(4) the availability of investment to implement OES at FORDA; and

(5) participants' perception of the level of their organisation's readiness for OES implementation.

Since this research involves human participants, before collecting data for the purpose of this research, the researcher had been given approval from the Human Ethics Committee (HEC) of the Victoria University of Wellington. Appendix 9 presents the HEC application. Initially, the researcher had sought approval and support from the research context in order to collect data from 20 regional offices at FORDA. The Secretary of FORDA had given consent for conducting the research by sending letters of approval and support to the heads of each regional office confirming that the researcher would contact the forestry directors directly for data collection. Thus, the researcher could appropriately access the research context. 


\subsubsection{Sampling}

Initially, the intention was to interview six policy makers from 20 regional offices at FORDA. To reach a total of six participants, interview kits were sent via email to the heads of nineteen regional offices. Each interview kit contained an information sheet about the study, a letter requesting participation in this study, interview procedure, and a participation consent form (Appendix 11), as well as an approval and support letter from the Secretary of FORDA for data collection. Follow-up emails and text messages were sent to the forestry directors within a week to set up interview appointments.

The researcher was present at the FORDA Annual Meeting which was held in Bali from 10 to 12 August 2008. Therefore, the survey kits and interview kits could be delivered directly to the heads of FORDA regional offices who attended the meeting. The researcher met the forestry directors in person and asked them to take part in the research as well as to address any issues regarding the survey. In total, twelve policy makers (forestry directors) from twelve regional offices were willing to take part in this research.

\subsubsection{The semi-structured interviews}

Before gathering data, the interview questions were checked by peer review and the research supervisor. The feedback was taken into consideration to improve comprehensiveness and clarity. An interview checklist was also prepared to standardise the interviews and to ensure that all the main questions had been asked and answered appropriately. Additionally, responses did not always follow the original sequence of the interview procedure as sometimes the interviewees covered the topics in a different order.

Each interview was transcribed thoroughly. To legitimately judge the credibility of the results the transcripts were sent back to each participant for verification, thus ensuring the validity of the data collected. The researcher could not predict the transferability of the findings; therefore, sufficient information about the research context was essential so that others could verify the applicability of the findings to a new situation (Lincoln \& Guba, 1985). To generalise or transfer the research findings to other contexts or settings, the research context and the assumptions that were fundamental to the research was described in detail. 
Twelve forestry directors from twelve regional offices were interviewed. Each of them was the head of their regional office. Of the twelve interviews, nine were conducted between 9 and 13 August 2008 in Bali. Due to limited time, two participants were interviewed a week later: one at his regional office, and one at his residence. Each interviewee was provided with an interview kit identical to the one which had already been sent to them, in case they had not received it. However, all forestry directors were aware of the research as they had already received the letter of approval and support from the Secretary of FORDA. A participation consent form was presented to each of them to be signed. All interviews were conducted by the researcher and on average each lasted for about 30 minutes. The interviews were recorded by using both digital and tape recorders. Additional notes were also taken to record unexpected new facts and which could be used to develop new questions to ask participants in other interviews. Although the researcher had planned to conduct a one-on-one interview with each participant, one respondent was accompanied by one of his staff. This is because the participant had only recently become the head of the regional office, thus the second person was able to provide additional information that the head might not know.

\subsubsection{Data preparation}

In the second phase of the research, the phenomenon being studied was examined from the perspective of policy makers. Data gathered were transcribed, codified and analysed thoroughly. Each transcript was analysed individually. The key points were highlighted and labelled. Subsequently they were put into different arrays to be categorised according to each main question or important topic being explored. The categorisation was conducted by placing facts within a matrix in a table by using Microsoft Word as suggested by Yin (1994). The questions were divided into three main categories: (1) the level of available and possible investment within each regional office; (2) potential challenges to OES implementation faced by each regional office; and (3) the critical factors that need to be developed in each research context to successfully implement OES. Each category required a different type of response. Table 34 shows eleven stages of data preparation approaches for the research analysis. 
Table 34. Data preparation for the research analysis

\begin{tabular}{|c|c|c|}
\hline Stage & Activity & Description \\
\hline 1 & Assessing the organisation & Seeking approval and support from the central office \\
\hline 2 & Contacting participants & $\begin{array}{l}\text { Sending a letter requesting participation and interview kits to nineteen FORDA regional } \\
\text { offices }\end{array}$ \\
\hline 3 & Profiling participants & $\begin{array}{l}\text { Gaining information and making important notes about each willing participant and the } \\
\text { research context }\end{array}$ \\
\hline 4 & $\begin{array}{l}\text { Making appointments for } \\
\text { interviews }\end{array}$ & Making a schedule for interviews \\
\hline 5 & Interviewing & Conducting twelve semi-structured interviews \\
\hline 6 & Transcribing the interviews & Listening to each participant's recorded interview and transcribing into writing \\
\hline 7 & $\begin{array}{l}\text { Validating the interview } \\
\text { transcripts }\end{array}$ & Sending back the transcripts to each participant for verification \\
\hline 8 & Open coding & $\begin{array}{l}\text { Analysing the text by using matrix (tables) in MS Word and commenting, labelling, } \\
\text { highlighting, and grouping the emergence of key facts (themes) }\end{array}$ \\
\hline 9 & Creating categories & Dividing concepts into three main categories \\
\hline 10 & $\begin{array}{l}\text { Integrating categories and } \\
\text { relationships }\end{array}$ & Integrating categories and finding relationships between them \\
\hline 11 & Writing up findings & $\begin{array}{l}\text { Generating hypotheses for future research, investigating little understood areas, and } \\
\text { Identifying/discovering important categories of meaning }\end{array}$ \\
\hline
\end{tabular}

Next, the data were tabulated and their relationships were examined (Yin, 1994). Finally, the information was put in sequential order to generate a plausible conclusion (Yin, 1994). The raw data was in the Indonesian language; therefore the data was prepared and analysed mostly using Indonesian. However, in some parts of the analysis stages, mostly in the last four stages (open coding, concept grouping, participants' speeches, etc.), the data were translated and analysed in English. At the final stage, English was used to present the findings, discussion, and interpretations.

\subsection{Step Two: The level of organisational readiness for OES}

All participants had positive attitudes about the possibility of implementing e-learning in their organisations. Almost all participants believed that e-learning is obligatory for a research and development organisation as it allows people to economically access and exchange information swiftly, effectively, and efficiently. In a large R\&D organisation such as FORDA, it is important to implement OES to support advanced learning as the core function of the organisation.

\subsubsection{Prior e-learning knowledge}

Three forestry directors believed that the nature of OES was not equally understood by employees across FORDA (Participants 2, 5, and 7). Yet there was no formal training for improving their knowledge about OES (Participants 3, 4, 5, 6, 7, 8, 9, 10, 11, and 12). Most employees improved their capacity through self-directed learning (Participants 2, 3, and 5). Some employees might not have been introduced to OES before. They may have not yet seen the benefit of OES; thus they do not perceive it as 
an interesting thing (Participant 11). In this regard OES training or introduction is necessary.

\begin{abstract}
"It should have been specific methods that need to be deployed in implementing elearning systems, so primarily we need to know [the methods]... Personally I am not really familiar with what the major requisites are for [implementing] e-learning systems; so I am keen to know more information [about e-learning]... Poor knowledge about elearning is the main factor that may hamper e-learning implementation, followed by limited human resources, and also I do not know how much we should spend on facilities and equipment or the amount of financial resources that we should have..." Participant 7
\end{abstract}

"So far formal training had not been delivered, but individually they [employees] had learnt by themselves to improve their capacity about e-learning. So even though only a minority, a few of them had properly used the available facilities [equipment, the Internet] to learn well" - Participant 5

Some programmes but no formal training had been set up to improve employees' capacity such as research schools, English courses, and online database training. However, these courses were not consistently delivered to all offices. This again suggests that prior e-learning knowledge is a significant factor within FORDA. The implementation of OES also seems important as it allows more people from dispersed locations to participate in the learning process. Training and development for employees is critically required to improve human resource capacity.

\footnotetext{
"So far, there was no specific training, but the central office has facilitated seminars for example an online database seminar, and in 2007 there had been a seminar about tips and guidelines of how to seek information and to have access to libraries or free electronic journals. However, only few employees can attend the seminar as it was held in Bogor" - Participant 3
}

These results confirm that e-learning is a relatively new concept to most people in a developing country like Indonesia. It appears that information about e-learning needs to be disseminated across FORDA. Although a large number of employees are Internet users, not all of them have in-depth knowledge about e-learning. E-learning need to be promoted across FORDA. This confirms that for a research context with no prior elearning knowledge, it is important to develop in-depth understanding about e-learning, so that the systems can be successfully initiated. 


\subsubsection{Human resources}

In general, the levels of qualifications were satisfactory across FORDA. Compared to branch offices, central offices were likely to have employees with a higher level of qualifications. However, in order to deploy successful OES, the human resources capacity was not sufficient. The results indicate that there was a lack of IT personnel at FORDA. Only a small number of regional offices had capable IT personnel (Participants 1, 5, and 9), and most had no IT personnel at all (Participants 2, 3, 4, 7, and 12).

\footnotetext{
"I did not see staff who are capable and enthusiastic [towards technology],... new recruitment is necessary, I have only one IT graduate employee, and he ends up dealing with all the applications or systems implemented within the organisation such as human resource information systems [HRIS] and institution accounting systems [IAS],... and that is impossible [for him to do the all the tasks by himself]..." - Participant 1

"I think human resource capacity should be developed gradually and constantly, because it is a new technology... information, and probably it is necessary to upgrade knowledge... because maybe not all are familiar with it [OES technology]..." Participant 11
}

Moreover, the study shows that there were very few potential champions (people who can facilitate and/or encourage employees to improve their self-development through OES) at FORDA.

These findings verify that current staff are not ready and able to implement successful OES. Thus, targeted professional development for developing and using OES need to be provided in order to implement sustainable OES as suggested by Nichols (2007).

\subsubsection{Attitude}

Overall, participants reported that their employees were enthusiastic about technology (Participants 2, 3, 6, 8, 11 and 12). They also believed that their employees were highly motivated and willing to improve their capacity through self-directed learning (Participants 2, 5, 6, 8, 9, and 11). Participant 2 claimed that about 50\% of employees who were familiar with the online environment were younger staff. In addition to that, $75 \%$ of the Internet users were researchers and research assistants; whereas about 25$30 \%$ of Internet users were managers and general employees. Participants 8 and 11 also clarified that technology is popular with most young employees. These statements verify that younger employees seem to be more technologically ready and have more enthusiasm for the online environment. 
Despite that, participants believed that a few of their employees were resistant to using OES technology. They might have concerns about the negative side of freely available information from the Internet (Participant 5). Moreover, to some degree, employees lack motivation to be engaged in an online environment: Participant 11 noted that although most employees were already familiar with Internet technology, they mostly used the Internet only for browsing information and sending email. Yet they had not been eager to be involved within online conferences or discussions (Participants 11 and 12).

\subsubsection{Culture}

The results confirm that culture significantly affects OES readiness in a developing country context. Participants believed that before developing and implementing OES, it is important to encourage the acceptance of online culture throughout FORDA (Participants 1, 2, 4, 8, and 12). Additionally, the study found that organisations in Asian countries tend to have more formal bureaucracies in their daily routines. Although most believed that face-to-face interaction is important, it is necessary to encourage self-awareness to change the conventional method to more online habits.

\footnotetext{
"It seems that culture is something that may slow down employees' adoption [of elearning]. It is perhaps because they [employees] are not accustomed to playing around using the Internet and so on, therefore it may be one factor that influences [slow adoption]..." - Participant 4
}

\footnotetext{
"We had tried to utilise a LAN and encourage people to communicate through it, but apparently it did not work. Because, perhaps, our culture is a written culture, with faceto-face habits... and formal traditions [for dealing and communicating with upper level managers]" - Participant 2
}

These results indicate that to some extent, familiarity with and adaptation to online habits or the online environment need to be improved as previously discussed in three other sections: prior e-learning knowledge, human resources, and attitudes. These confirm the findings of a study by Nichols (2007) suggesting that in order to achieve a sustainable OES, organisational culture needs to be ready.

\subsubsection{Equipment and infrastructure - Internet connection}

The most noticeable barrier to OES implementation was Internet connection. The level or availability of Internet connection was quite varied across FORDA. Only a few offices - mostly central and nearby - had 24/7 Internet access through wireless hotspots 
and/or ASDL. However, one office had only one landline which was used for both office phone and Internet access. Three remote offices were only connected through satellite. Some offices still had very limited Internet access. Accordingly, most areas are prone to problems such as poor connection speed, low Internet capacity (Participants 1, $3,4,6,7$, and 9) and costly Internet connection.

Equipment was not a significant concern for a few offices. Considerable investment had been made to set up Internet connection in three very remote regional offices. However, one of the participants said that this advanced technology has not been yet utilised maximally (Participant 11). Whilst the other two participants (Participants 3 and 4) said that obstructions were still common such as good signals relying on weather conditions and insufficient infrastructure (electricity supply). Participant 10 mentioned that FORDA needs to support the rest of its regional offices to have this advanced equipment as well. This implies that FORDA need to improve the Internet connection across regional offices.

On average the availability of software, hardware and other facilities were moderately sufficient across FORDA (Participants 1, 2, 5, 8, and 11). Yet facilities needed to be improved or upgraded constantly in all offices (Participant 5). Participant 6 said that there were insufficient hardware, very limited facilities, and an urgency to develop Internet connection. To some degree the infrastructure were quite poor (Participants 7 , 8 , and 9). It seems that only two central offices and three remote regional offices had advanced LAN, whilst most regional offices had basic LAN. This means that equipment including hardware, software, and other facilities supporting OES, need to be continually improved to keep up to date with the constantly changing environment.

Furthermore, power supply was an obvious external obstruction particularly for most offices in remote areas. Some areas have limited and unreliable electricity supply (Participants 4 and 6). One regional office could not occupy the new building that has been built recently as there was no power line in the new office location (Participant 1).

“...electricity supply is a problem. I can afford to buy computers, financially it's not a problem, the central office can provide financial support for that, however apparently the power supply is insufficient, I want to improve the power capacity but it is not available. So we have [can only operate] limited computers..." - Participant 1 
Participant 10 mentioned that their office building was still under construction, and they were temporarily operating in one of the Ministry of Forestry offices. Thus, it seems that very little information can be found to assess the level of readiness of this particular research context. Participant 4 also said that their office was housed in five buildings, thus, Participant 4 thought that the network capacity was to some extent inappropriately distributed.

It seems that although financial and equipment support from the central office were adequate, external barriers such as scarce electricity supply, poor infrastructure, office construction, and weather conditions may obstruct OES implementation.

\subsubsection{Financial support}

Lack of financial support was revealed by most participants (Participants 1, 2, 3, 4, and 9). The results show that there were insufficient funds for software maintenance such as upgrading antiviral software (Participant 2), and systems updating and facilities maintenance (Participants 1, 2, 3, 4 and 9). There was also an indication of lack of financial readiness for facilities and equipment establishment, upgrading, maintenance, and operational costs. These conditions confirm that the level of financial readiness is low across FORDA; therefore it is critical to improve the financial resources. As other studies have found, cost is the biggest impediment for initiating, implementing, and maintaining e-learning (Ali \& Magalhaes, 2008; Bell, Martin, \& Clarke, 2004; Welsh et. al., 2003).

\subsubsection{Management support}

To some extent there was a lack of policy support from the upper level management. No policy makers have taken any initiative in promoting OES to be implemented at FORDA. It was suggested that a centralised pilot e-learning community needs to be set up immediately (Participants 4, 8, and 11).

Although an IT department was seen as important (Participant 3), there was no specific unit dedicated to managing or maintaining IT within regional offices across FORDA. Thus, before implementing OES, it is important to consider that an IT department needs to be established across regional offices at FORDA to provide appropriate support. 
The results indicate that to encourage employees to be involved in OES, incentives could be given in many different forms including: providing advanced and up-to-date equipment or facilities such as hardware and software technology so that people can communicate with their peers (Participants 2, 3, 10, 11, and 12); improving infrastructure including Internet connection (Participants 1, 2, and 11); giving rewards and recognition (Participants 1 and 4); improving skills and competency by facilitating training (Participants 4, 5, 7, and 12); providing financial incentives (Participants 2 and 4); giving it an important role (Participant 5); and facilitating or budgeting the programme development (Participants 3 and 7).

However, other participants believed that incentives such as financial stimulus were not necessary. They noted that OES provide a great opportunity for them, particularly for researchers and research assistants to learn; thus, this great advantage was seen as sufficient incentive (Participants 6, 7, 8, and 9). Participant 9 added that incentives could also be in the form of benefits that the systems provide including the system allowing them to perform their routines (e.g. accessing, delivering, and disseminating information) efficiently and effectively. Participants also believed that those who cannot embrace OES will be left behind in this field.

Overall, FORDA needs to improve central support, and the role of upper level management is critical to successfully implement OES. This verifies that sufficient support from senior management is vital to successful OES implementation (Nichols, 2007).

\subsection{Step Three: Barriers to OES implementation}

Several barriers that may slow down employees' adoption of OES were identified. The obstructions were varied across FORDA. The results confirm that age was seen as one of the barriers. Other barriers that slow down employees' adoption are the fact that some job descriptions do not require employees to be in touch with online technology, and that employees tend to perform tasks that are likely to only benefit their own career or specific needs (Participant 8). Lack of English proficiency (Participants 3, 6, and 11) and poor communication skills (Participant 6) were also seen to hinder employees' adoption. Lack of willingness to learn (Participants 1, 3 and 7), low education levels 
(Participant 7), and lack of interest in e-learning (Participants 2, 8, and 11) were also considered as impediments to adoption.

\begin{abstract}
"age might be a barrier of adoption... learning could be difficult for those above 45 or 50, however for us under 40, it seems that the curiosity or willingness to learn new skills involving e-learning is very important, without being asked, they learn by themselves to be able to ... so age is a problem" - Participant 2
\end{abstract}

Difficulties of controlling and maintaining the online environment were also mentioned by several participants (Participants 1,2,3,8, and 11). The problems include privacy and security breaches as a result of inappropriate firewall protection (Participants 2 and 8), viruses (Participant 3), and the ability to filter the abundance of information on the Internet and to decide what is appropriate or relevant (Participant 3). Poor information maintenance was also revealed as some websites do not provide the information required or up-to-date information (Participant 9).

\footnotetext{
"There is an absurd concern, I think... but it could be true or false, that some people may perceive e-learning leads to the leak of classified issues or confidential data. It is about how each person deals with it. Some believe that it is better to spread information rather than it being stolen by others, it could be leaked but still in a controlled way... therefore, this may be a challenge in the future... of how to overcome this..." - Participant 11
}

These obstacles indicate that FORDA needs capable personnel to manage and maintain the online environment. Certain policies should be addressed prior to dealing with privacy, security, and legal issues.

Participants were asked to name the top three barriers to implementing OES within their regional offices. Eleven obstacles emerged during the interviews (Table 35). A lack of human resources capacity was ranked first by four participants, followed by limited knowledge about e-learning, which was ranked first by three participants. Afterwards, two participants ranked inadequate facilities/equipment first. However, the three most common barriers given by participants were inadequate human resources, followed by inadequate facilities/equipment, and limited OES knowledge. Other barriers are also included in Table 35. 
Table 35. Top three barriers for implementing OES in twelve regional offices at FORDA

\begin{tabular}{|c|c|c|c|c|c|c|c|c|c|c|c|c|}
\hline Participants & 1 & 2 & 3 & 4 & 5 & 6 & 7 & 8 & 9 & 10 & 11 & 12 \\
\hline 1. inadequate knowledge about OES & & & & & 1 & & 1 & 1 & & 3 & & \\
\hline 2. Inadequate facilities/equipment/hardware/software & & 3 & 2 & & 2 & 1 & & 2 & 1 & & & 2 \\
\hline 3. Lack of human resources & 1 & 1 & & 3 & & & 2 & & 2 & 1 & & 1 \\
\hline 4. $\quad$ Poor Internet connection/capacity/speed & 2 & & & 1 & & & & & 3 & & & 3 \\
\hline $\begin{array}{l}\text { 5. Poor infrastructure (electricity supply, landline, office } \\
\text { building construction) }\end{array}$ & 3 & & & 2 & & & & & & 2 & 1 & \\
\hline 6. Lack of financial support & & 2 & & & & 2 & 3 & & & & & \\
\hline 7. Poor English skills & & & 3 & & & 3 & & & & & 3 & \\
\hline 8. Resistant to using technology & & & & & 3 & & & & & & & \\
\hline $\begin{array}{l}\text { 9. Not all job descriptions require employees to use } \\
\text { online technology }\end{array}$ & & & & & & & & 3 & & & & \\
\hline 10. Unwillingness to learn & & & 1 & & & & & & & & & \\
\hline 11. Unwillingness to be involved in an online environment & & & & & & & & & & & 2 & \\
\hline
\end{tabular}

\subsection{Summary}

Overall the findings indicate that FORDA has the ability to implement OES, particularly in their attitude towards OES. However, most dimensions still need to be developed further including technological skills, online learning styles, equipment and infrastructure, human resources capacity, culture, environment, and finance. Of these dimensions, human resources, equipment/infrastructure, and finance were considered critical factors that need to be addressed further in implementing successful OES. In addition, one important finding was that prior knowledge about OES needed to be spread across FORDA. Central support, and the role of upper level management were also critical to successfully implement OES. 


\section{Chapter 6: Conclusions}

This final chapter provides a closure of the study. Firstly, it explains the integration of discussion and interpretations of the findings from both quantitative and qualitative analyses to address each of the three main research questions. Then, the integration and comparison between quantitative results and qualitative findings are discussed. The contributions, limitations, implications, and future directions of this research are also examined subsequently.

This chapter is organised as follows:

6.1 The research findings: Addressing the research questions

6.2 Contributions

6.3 Implications

6.4 Limitations

6.5 Directions for future research

\subsection{The research findings: Addressing the research questions}

The three main research questions were addressed by using a mixed-method approach. A brief summary of each approach used and the findings is presented below.

\subsubsection{Research question 1: What is the level of organisational readiness for implementing e-learning systems in FORDA?}

The level of organisational readiness for OES was assessed by using both quantitative and qualitative analyses. The quantitative results show that overall five areas are ready but need a few improvements at FORDA: equipment/infrastructure, attitude, environment, positive online culture, and financial availability. Three areas are not ready and need some work for improvement: technological skills, positive online learning style, and human resources. Moreover, the areas that are not ready and still need a lot of work for improvement are: financial affordability, and financial possibility. As discussed in the previous chapter, two dimensions, negative online learning style and negative culture explain conventional learning style and conventional culture rather than online learning style and online culture. The readiness percentages in 
these two areas were nearly 50\%. This implies that the level of readiness in online learning style and online culture still need to be improved.

On the other hand, the qualitative findings show that FORDA was most ready in terms of attitude. The findings also demonstrate that the other dimensions still need to be improved. Three dimensions were considered the most critical: human resources, equipment/infrastructure, and finance. Furthermore, one significant finding was revealed which is the importance of prior knowledge about OES which can affect successful OES implementation.

\subsubsection{Research question 2: What are the critical factors that need to be considered in order to implement successful e-learning systems in FORDA?}

The critical factors for implementing successful OES were determined by using both quantitative and qualitative analyses. The second research question has been answered by addressing the first research question of determining the level of organisational readiness beforehand. Thus, the critical factors that need to be considered in order to implement successful OES at FORDA could be identified.

The quantitative results revealed five critical factors or areas that are not ready: three areas are not ready and needs some work for improvement (technological skills, positive online learning style, and human resources), and two areas are not ready and still need a lot of work for improvement (financial affordability, and financial possibility). The scores of readiness in these five areas were particularly low. Thus, they were considered critical factors that FORDA should make priorities in order to implement successful OES. Of these five factors, finance is the most critical as it had the lowest scores of all. In addition, the results confirmed that age, education, and Internet access at work were significant predictors of the overall perception of their organisation's readiness for OES.

Overall, the qualitative findings verify the quantitative results. In addition, the qualitative phase added that all dimensions except attitude readiness were believed to require improvement. Three critical barriers were identified including: inadequate human resources, inadequate facilities/equipment, and finance. The important factor of limited OES knowledge was also identified. 


\subsubsection{Research question 3: What are the key factors that influence organisational readiness for OES in an organisation with no prior e-learning knowledge in a developing country context?}

Both quantitative and qualitative analyses were used to determine the key factors that influence organisational readiness for OES particularly in a research context with no prior e-learning knowledge. The research question was addressed by examining and comparing the results and findings from both analyses.

Aydin and Tasci (2005) claimed that personal characteristics (gender, age, education levels, and computer experience) have no effect on respondents' perceptions of organisational readiness for e-learning. Conversely, the quantitative results in this study reveal that age, education, and gender are important factors that influence readiness towards OES at FORDA. Both quantitative and qualitative findings verify that older learners had lower technical skills and computer self-efficacy than younger learners as confirmed by Pillay et al. (2007). It seems that these factors need to be considered in a research context with no prior e-learning knowledge. This study also shows that respondents' computer experience and internet access are important factors that affect organisational readiness for e-learning. Figure 22 presents significant predictors that influence OES readiness from twelve dimensions.

Aydin and Tasci (2005) revealed that in order to successfully implement e-learning in emerging countries, human resources in particular need to be improved. Both quantitative and qualitative findings in this study confirm that human resources was a key factor that needs to be dealt with thoroughly. In addition, Sadik (2007) identified barriers for e-learning implementation including lack of knowledge, lack of training, and lack of technical support. The qualitative findings confirm that the lack of effective human resources readiness can easily be associated with the current status of human resources training programmes at FORDA. Currently, there is no specific programme focusing on online training. There is also a lack of understanding about e-learning. 


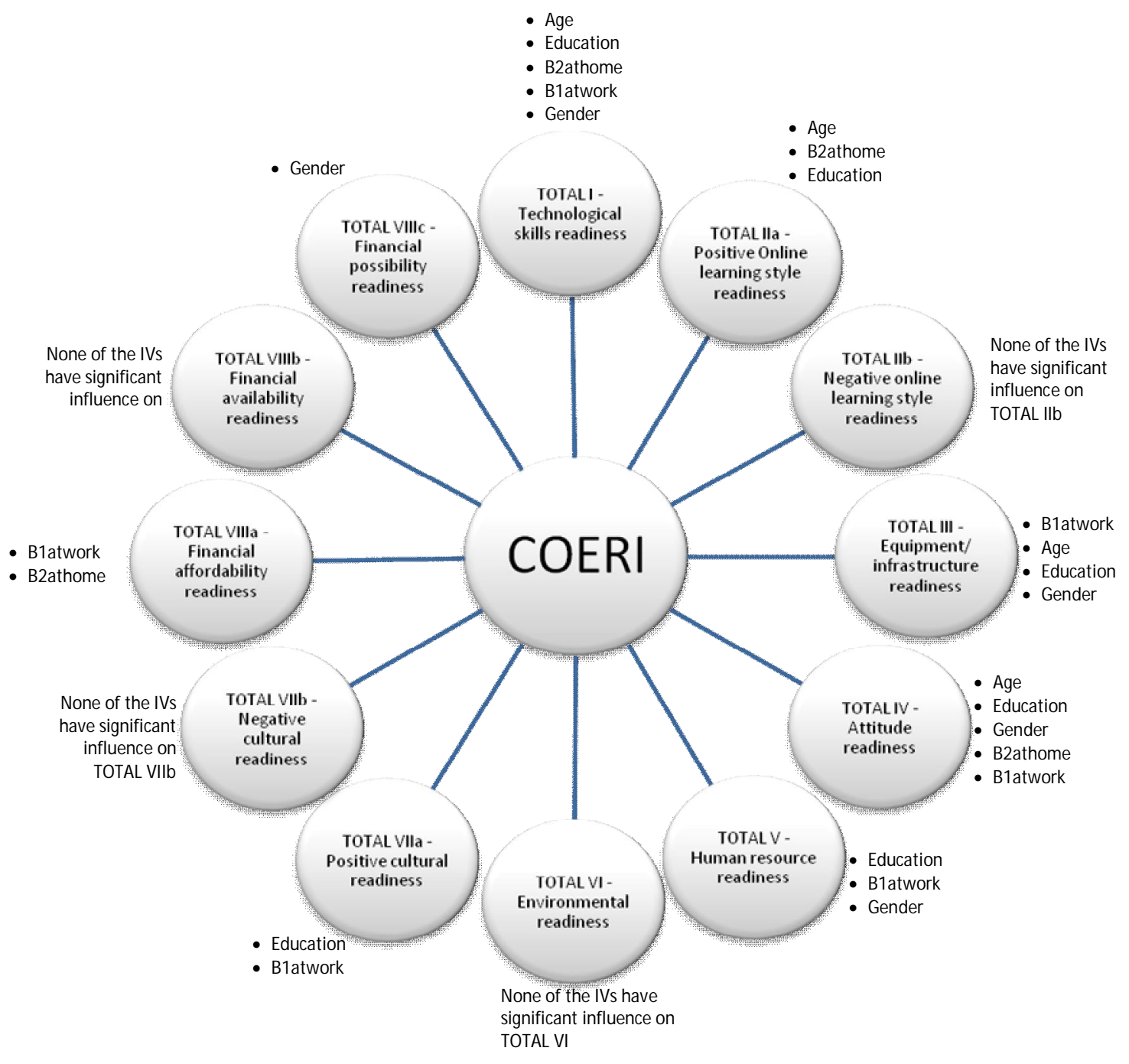

Figure 22: Significant predictors that influence e-learning readiness in an organisation with no prior elearning knowledge - quantitative results

English proficiency was seen as a factor that hampers OES implementation in Malaysia (Abas, Kaur, \& Karun, 2004). The qualitative findings confirm that poor English proficiency and poor communication skills hamper OES implementation in Indonesia. This suggests that this problem is common in such research contexts particularly where English is not the first language.

Another impediment that is commonly found in a developing country context with no prior OES knowledge was the inadequacy of equipment and infrastructure including poor access to the Internet. This study identifies this impediment from both analyses and thus confirms a study by Sadik (2007). 
It seems that nurturing online culture and making staff familiar with online learning styles are key factors that influence organisational readiness for OES particularly in a context with no prior OES knowledge. Both the survey results and the qualitative findings show that online culture was ready but needs a few improvements. Additionally, the survey results reveal that online learning styles was not ready and needs some work for improvement; the qualitative findings also reveal that this dimension still needs to be improved to some degree.

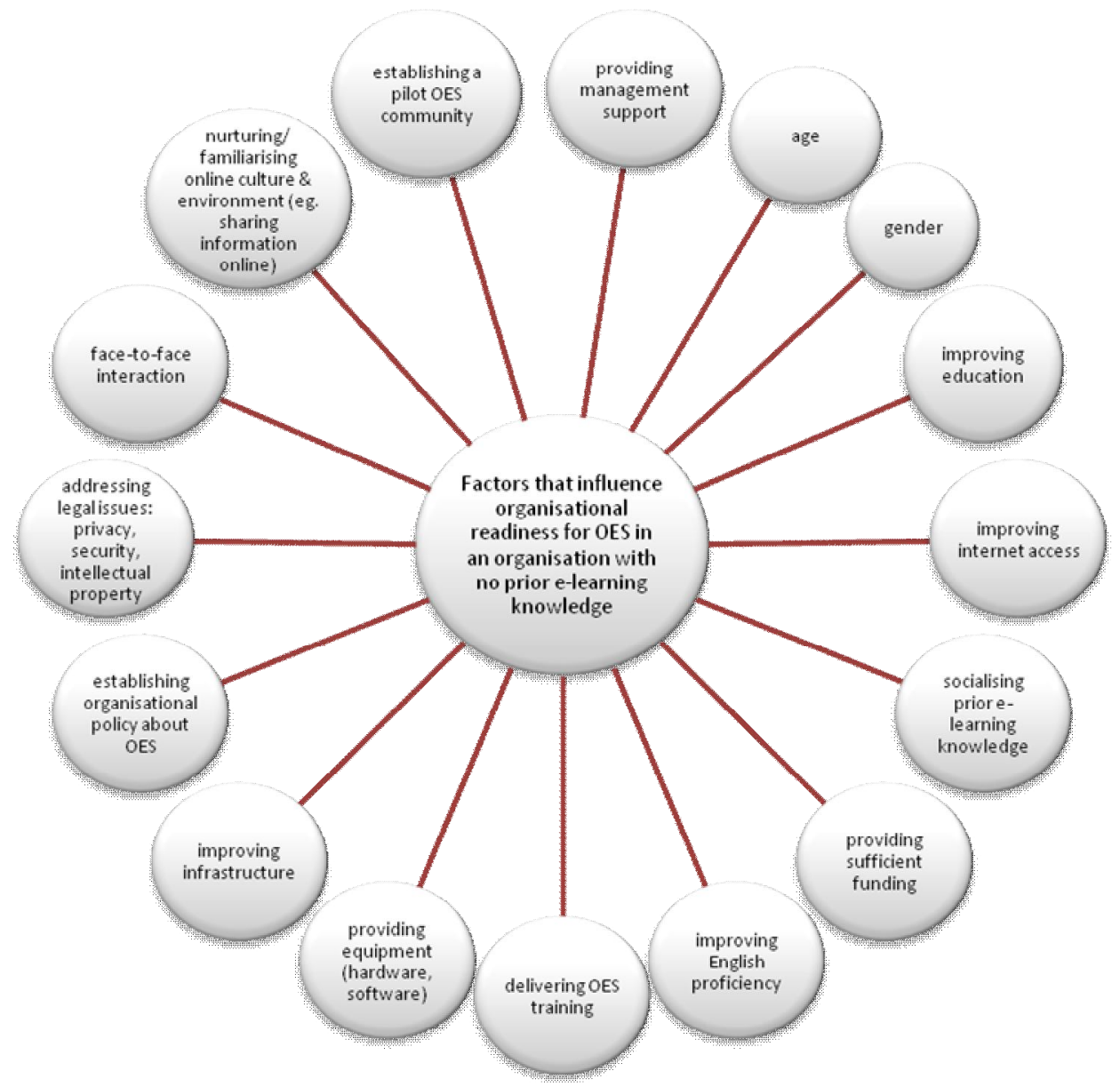

Figure 23: Factors that influence organisational readiness for OES in a developing country context with no prior e-learning knowledge - quantitative results supported by qualitative findings

However, the study confirms that face-to-face is still the preferred way to deliver training. The survey results indicate that the most preferred channel of communication for learning was face-to-face (62.2\%), followed by email (29.2\%), SMS (21.9\%), chat on the internet (22.9\%), and written memo (35.1). The least preferred medium was postal mail (40.6\%). Moreover, the qualitative findings also show that face-to-face cannot be totally removed from a learning process. Figure 23 provides the key factors from both analyses and depicts the summary of factors that influence organisational readiness in a research context with no prior e-learning knowledge. 
Finance is the most important factor that affects successful OES implementation not only in developing countries, but also developed countries. The survey results show that finance was the lowest dimension of readiness. The qualitative findings also indicate that financial support needs to be improved considerably across FORDA particularly to provide investment for initiating OES. Finance was to some extent related to other dimensions of readiness. For example, finance was crucial to develop equipment and infrastructure, to improve human resources capacity, and to improve motivation and attitudes towards OES. Therefore, all stakeholders will be involved and affected by financial readiness. This confirms previous studies which suggested financial readiness of the organisation is predicted to be a key factor by all stakeholders (Abas, Kaur, \& Karun, 2004; Chapnick, 2000; Karmakar \& Wahid, 2006).

The study confirms that environmental readiness for OES is also one of the key factors that affect successful OES implementation in a research context with no prior OES knowledge. This factor may include: inadequate organisational policies to support OES, legal issues, privacy and security issues, and intellectual property.

Finally, central support is also seen as a key factor that affects successful OES implementation. Without it, it seems impossible to initiate the development and implementation of OES.

\subsubsection{Summary}

Overall, to implement OES at FORDA the quantitative results show that only five areas are ready but need a few improvements, and five areas are not ready with three areas needing some improvements and two areas needing major improvements. The qualitative findings confirm that most dimensions need to be improved further. Figure 24 shows the summary of comparison between quantitative and qualitative findings. The comparison between the two phases indicates that all areas of readiness had similar results. The qualitative findings reveal that three areas considered critical were human resources, equipment/infrastructure, and finance. Both quantitative and qualitative results show that there was a lack of human resources readiness. Both analyses also indicate that financial support was seen as insufficient at FORDA. 


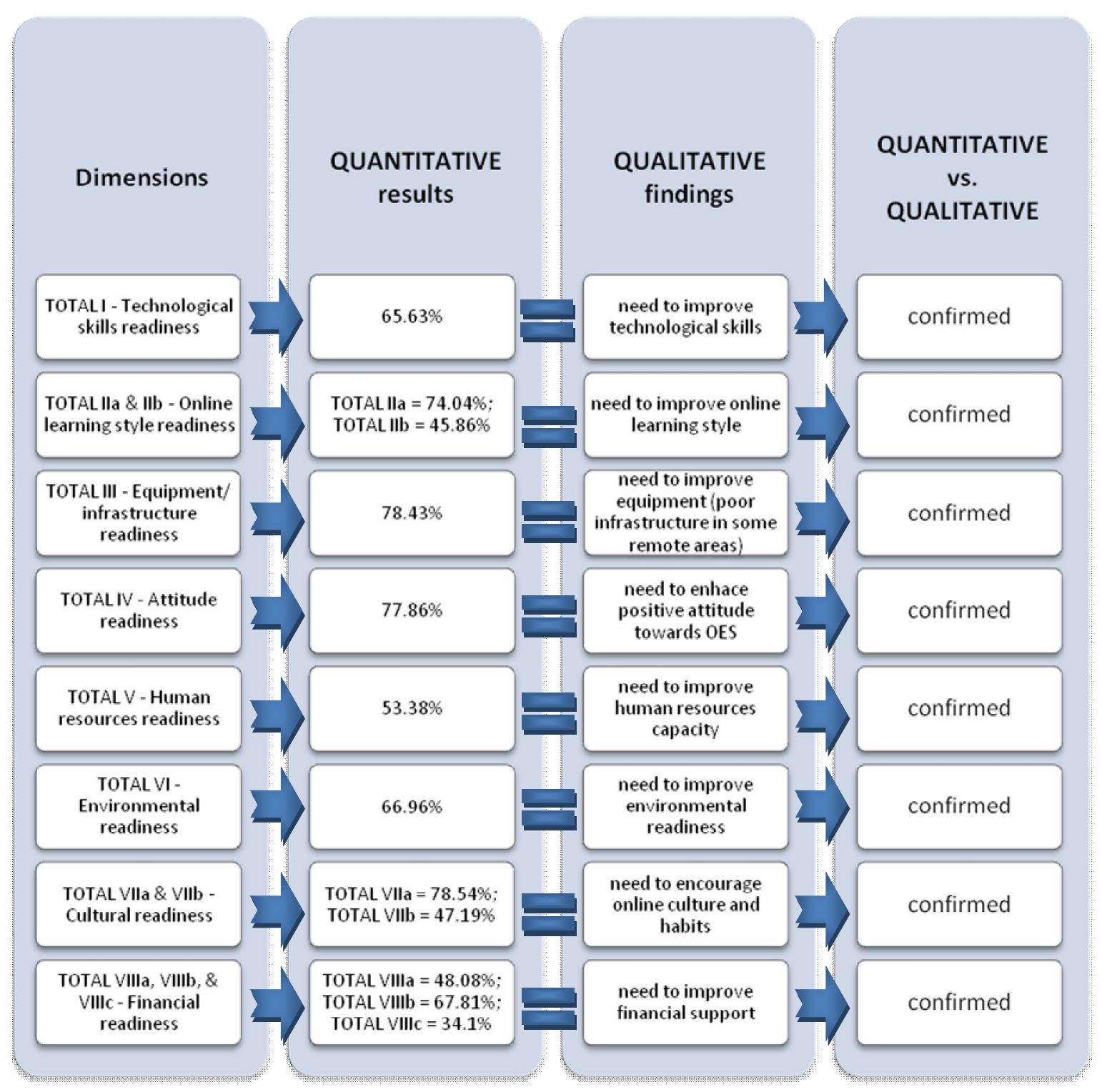

Figure 24: Summary of comparison between quantitative results and qualitative findings

Both findings indicate that technological skills and online learning style readiness need to be improved considerably. The survey results show that equipment/infrastructure was overall ready and needs a few improvements, while the qualitative findings confirm that equipment/infrastructure is one of the critical areas that may need some work for improvement particularly in terms of inadequate facilities and equipment. Furthermore, one additional critical factor revealed by the qualitative phase was limited prior knowledge about OES. 
The percentage of organisational readiness for OES at FORDA is presented in Figure 21 (p. 93). At this stage, overall FORDA is not ready for OES implementation on a nationwide basis. However, it is possible to pilot an OES project in one or two offices especially where the technological and human resources are more ready. Funding for this pilot project may be found from global funding agencies e.g. Asian Development Bank, etc.

\subsection{Contributions}

This study provides a number of contributions for both academics and practitioners in the information systems field. Firstly, this research provides a comprehensive measurement to examine the level of readiness for initiating, developing, and implementing OES particularly in a research context with no prior e-learning knowledge.

Secondly, COERI offers a multidimensional interpretation of measuring e-learning readiness by providing twelve dimensions to examine all stakeholders. Watkins et al. (2004) noted that there is a need to measure readiness from multiple perspectives which could be vital in supporting the broad use of the instrument as a predictive and prescriptive tool for those considering e-learning opportunities. COERI was designed to assess multiple perspective of e-learning readiness.

Thirdly, COERI extends TSROL (Pillay et al, 2007) through positive online learning style and attitude readiness scales by providing more dependable results. In particular they improve the reliability of learning preferences and attitudes towards computers presented within TSROL.

Finally, this study successfully deployed a mixed-methods approach with regard to the research design and methodology used. This approach was conducted concurrently but not in the same phase. The qualitative phase was conducted in the same period of time to support the main quantitative phase. Triangulation verified the findings, as each method improved and confirmed the other. 


\subsection{Implications}

A number of more general implications of this study are identified. Firstly the research has promoted the benefit of a comprehensive survey instrument that can be used to assess organisational readiness for OES implementation for different stakeholders. Thus, the instrument offers a much less cumbersome assessment compared to other instruments that assess each stakeholder differently. The study also provides twelve dimensions to assess OES readiness which are customised to a research context with no prior OES knowledge.

Finally, the study reveals critical factors that need to be developed further to implement OES in a developing country context. Moreover, it identifies key factors that influence organisational readiness for OES in an organisation with no prior e-learning knowledge in a developing country context. Thus, these can be used for helping policy makers and other stakeholders who are keen to embrace OES to make decisions, particularly those in similar circumstances to the research context.

\subsection{Limitations}

Several limitations were identified in this study. Firstly, the mixed-methods research was a complicated approach that requires considerable time and effort. This study, however, was completed in about fourteen months. Therefore, although the researcher attempted to conduct the research in an acceptable manner, it is still prone to flaws.

Secondly, English was not the first language in the research context. Therefore, the researcher had to provide translations during the process of data collection and data analysis. The interviews and surveys were conducted in the Indonesian language; thus it took significant effort and time. Moreover, some cultural contexts in the use of both languages might not be able to be interpreted accurately. Although the researcher attempted to be as accurate as possible, biases or misinterpretations were possible.

Finally, the COERI developed was not perfect. It needs to be improved to achieve a more reliable instrument and to be tested to increase the applicability of the instrument. For example, two items within the financial possibility scale (C.VIII.6 and C.VIII.7) are negatively worded questions. However, they were not reversed during the analysis. This 
implies that the result should be read the other way around. In other word, the level of readiness in financial possibility was actually the lowest at about $34.1 \%$.

\subsection{Directions for future research}

As mentioned before, the COERI is a first step. Further improvement is required to achieve a more reliable instrument and to increase the applicability of the instrument in other research contexts which have similar circumstances. Further explorations should be performed, particularly in some scales and/or subscales that have low scores of internal consistency, to achieve a more reliable instrument. The scales and subscale after factor analysis also need to be investigated further. Moreover, some of the survey questions need to be addressed, particularly those with negatively worded questions, to minimise the risk of respondents answering the question wrongly.

Although the data was carefully collected, the issue of English proficiency for translation of the questionnaire and the interview questions should be addressed in future research. Additionally, communication and education jargon were used without considering clarity for all respondents (e.g. Question B5). Therefore, future research should limit the use of jargon within the COERI so it can be understood by all participants.

Finally, the data gathered from both methods were valuable and quite substantial. However, not all of them were used in the study. Thus, it could be useful to conduct further analysis as it may generate valuable information for future research.

\section{References}

Abas, Z.W., Kaur, K. \& Harun, H. (2004). E-learning Readiness in Malaysia 2004. A Join Study by the Ministry of Energy, Water and Communications (MEWC), Malaysia and Open University Malaysia (OUM).

Ali, G.E. \& Magalhaes, R. (2008). Barriers to implementing e-learning: A Kuwaiti case study. International Journal and Development, 12(1), 36-53.

Aydin, C.H. \& Tasci, D. (2005). Measuring readiness for e-learning: Reflections from an emerging country. Educational Technology \& Society, 8(4), 244-257. 
Bartlett II, J.E., Kotrlik, J.W., \& Higgins, C.C. (2001). Organizational research: Determining appropriate sample size in survey research. Information Technology, Learning, and Performance Journal, 19(1), 43-50. http://www.osra.org/itlpj/bartlettkotrlikhiggins.pdf

Bell, M., Martin, G., \& Clarke, T. (2004). Engaging in the future of e-learning: A scenarios-based approach. Education + Training, 46(6/7), 296-307.

Bohl, O. \& Scheer, A.W. (2007). A case study on education networks and brokerage. Sharma, R.C. \& Mishra, S. (Eds.), Cases in Global E-Learning Practices: Successes and Pitfalls (pp. 35-51). Hershey, Pennsylvania: Idea Group Inc.

Chan, S.C.H. \& Ngai, E.W.T. (2007). A qualitative study of information technology adoption: How ten organizations adopted Web-based training. Information Systems Journal, 17(3), 289-315.

Chapnick, S. (2000). Are you ready for e-learning? Learning Circuits: ASTD's Online Magazine All About ELearning. http://www.learningcircuits.org/2000/nov2000/Chapnick.htm

Cohen, J. (1992). Statistical power analysis. Current Directions in Psychological Science, 1(3), 98-101.

http://helicon.vuw.ac.nz/login?url=http://search.ebscohost.com/login.aspx?direct=t rue $\& \mathrm{db}=$ pbh $\& \mathrm{AN}=10768783 \&$ site $=$ ehost-live

Condie, R. \& Livingston, K. (2007). Blending online learning with traditional approaches: Changing practices. British Journal of Educational Technology, $38(2), 337-348$.

Cook, D.A. \& Dupras, D.M. (2004). A practical guide to developing effective Webbased learning. Journal of General Internal Medicine, 19(6), 698-707.

Creswell, J.W. \& Plano-Clark, V.L. (2007). Designing and Conducting Mixed Methods Research. Thousand Oaks, California: Sage Publications.

Creswell, J.W. (2003). Research Design: Qualitative, Quantitative, and Mixed Method. $2^{\text {nd }}$ ed. Thousand Oaks, California: Sage Publications. 
Cronbach, L. J. (1951). Coefficient alpha and the internal structure of tests. Psychometrika. 16(3), 297-334.

Davis, H.C. \& Fill, K. (2007). Embedding blended learning in a university's teaching culture: Experiences and reflections. British Journal of Educational Technology, $38(5), 817-828$.

DeLone, W.H. \& McLean, E.R. (2004). Measuring e-commerce success: Applying the DeLone \& McLean Information Systems Success Model. International Journal of Electronic Commerce, 9(1), 31-47.

Economist Intelligence Unit Limited \& IBM Corporation. (2003). The 2003 e-learning readiness. http://graphics.eiu.com/files/ad_pdfs/eReady_2003.pdf

Economist Intelligence Unit Limited \& IBM Corporation. (2008). E-readiness rankings 2008: Maintaining momentum. http://a330.g.akamai.net/7/330/25828/20080331202303/graphics.eiu.com/upload/i bm_ereadiness_2008.pdf

Field, A. (2000). Discovering Statistics using SPSS for Windows. Thousand Oaks, California: Sage Publications.

Field, A. (2005). Discovering Statistics using SPSS. $2^{\text {nd }}$ ed. Thousand Oaks, California: Sage Publications.

Golafshani, N. (2003). Understanding reliability and validity in qualitative research. The Qualitative Report, 8(4) 597-607.

Haney, D. (2002). Assessing organizational readiness for e-learning: 70 questions to ask. Performance Improvement, 41(4), 8-13.

Holsapple, C.W. \& Lee-Post, A. (2006). Defining, assessing, and promoting e-learning success: An information systems perspective. Decision Sciences Journal of Innovative Education, 4(1), 67-85. http://www.blackwellsynergy.com/doi/pdf/10.1111/j.1540-4609.2006.00102.x

Israel, G.D. (2003). Determining sample size. The Agricultural Education and Communication Department, Florida Cooperative Extension Services, Institute of 
Food and Agricultural Sciences, University of Florida, 1-5.

http://edis.ifas.ufl.edu/PD006

Kaczynski, D., Wood, L., \& Harding, A. (2008). Using radar charts with qualitative evaluation: Techniques to assess change in blended learning. Active Learning in Higher Education, 9(23), 23-41.

http://alh.sagepub.com/cgi/content/abstract/9/1/23

Karmakar, C. K. \& Wahid, C.M.M. (2006). Recommendations for Bangladesh Towards E-Learning Readiness. http://www.codewitz.net/papers/MMT_97102_Karmakar_Wahid\%20.pdf

Lincoln, Y. \& Guba, E. (1985). Naturalistic Inquiry. Beverly Hills, California: Sage Publications.

Maglogiannis, I. \& Karpouzis, K. (2007). Combining synchronous and asynchronous distance learning for adult training in military environments. In Sharma, R.C. \& Mishra, S. (Eds.), Cases in Global E-Learning Practices: Successes and Pitfalls (pp. 23-34). Hershey, Pennsylvania: Idea Group.

Mathieson, M. (2006). Improving organisational performance through developing our people. Industrial and Commercial Training, 38(2), 70-77.

Meyer, P. B. (2009). Online Glossary of Research Economics: Definition of Cronbach's Alpha. Retrieved April 20, 2009, from http://economics.about.com/cs/economicsglossary/g/cronbachalpha.htm

Mutula, S.M. \& Brakel, P.V. (2007). ICT skills readiness for the emerging global digital economy among small businesses in developing countries: Case study of Botswana. Library Hi Tech, 25(2), 231-245.

National Institute of Standards and Technology (NIST)/SEMATECH. (2006). EHandbook of Statistical Methods: Critical values of the chi-square distribution. http://www.itl.nist.gov/div898/handbook/eda/section3/eda3674.htm

Nichols, M. (2007). Institutional perspectives: The challenges of e-learning diffusion. British Journal of Educational Technology. http://www.blackwellsynergy.com/doi/pdf/10.1111/j.1467-8535.2007.00761.x 
Nicholson, P. (2007). A history of e-learning. In Manjón, B.F., Pérez, J.M.S., Pulido, J.A.G., Rodríguez, M.A.V., \& Rodríguez, J.B. (Eds.), Computers and Education: E-learning, from Theory to Practice (pp. 1-9). Dordrecht: Springer.

Pallant, J. (2001). SPSS Survival Manual: A Step by Step Guide to Data Analysis Using SPSS for Windows. Maidenhead: Open University Press.

Pallant, J. (2007). SPSS Survival Manual: A Step by Step Guide to Data Analysis Using SPSS for Windows. 3rd ed. Maidenhead: Open University Press.

Pillay, H., Irving, K., \& Tones, M. (2007). Validation of the diagnostic tool for assessing tertiary students' readiness for online learning. Higher Education Research \& Development, 26(2), 217-234.

Rhee, B.V.D., Verma, R., Plaschka, G.R., \& Kickul, J.R. (2007). Technology readiness, learning goals, and e-learning: Searching for synergy. Decision Sciences Journal of Innovative Education, 5(1), 127-149.

Sadik, A. (2007). The readiness of faculty members to develop and implement ELearning: The case of an Egyptian university. International Journal of ELearning, 6(3), 433-453.

Santos, J. R. A. (1999). Cronbach's Alpha: A Tool for Assessing the Reliability of Scales. The Journal of Extension, 37(2), 1-5. http://www.joe.org/joe/1999april/tt3.php

Sharma, R.C. \& Mishra, S. (2007). Cases in Global E-Learning Practices: Successes and Pitfalls. Hershey, Pennsylvania: Idea Group Inc.

Smith, P.J. (2005). Learning preferences and readiness for online learning. Educational Psychology, 25(1), 3-12.

Stokes, C.W., Cannavina, C., \& Cannavina, G. (2004). The state of readiness of student health professionals for web-based learning environments. Health Informatics Journal, 10(3), 195-204. http://jhi.sagepub.com/cgi/content/abstract/10/3/195

UCLA Academic Technology Services [UCLA-ATS]. SPSS FAQ: What does Cronbach's alpha mean? Retrieved April 20, 2009, from http://www.ats.ucla.edu/stat/Spss/faq/alpha.html 
Unneberg, L. (2007). Grand designs for e-learning - can e-learning make the grade for our biggest corporates? Industrial and Commercial Training, 39(4), 201-207.

Watkins, R., Leigh, D., \& Triner, D. (2004). Assessing readiness for e-learning. Performance Improvement Quarterly, 17(4), 66-79.

Welsh, E.T., Wanberg, C.R., Brown, K.G., \& Simmering, M.J. (2003). E-learning: Emerging uses, empirical results and future directions. International Journal of Training and Development, 7(4), 245-258.

White, S. (2007). Critical success factors for e-learning and institutional change - some organisational perspectives on campus-wide e-learning. British Journal of Educational Technology, 38(5), 840-850.

Wild, R.H., Griggs, K.A., \& Downing, T. (2002). A framework for e-learning as a tool for knowledge management. Industrial Management \& Data Systems, 102(7), 371-380.

Yin, R.K. (1994). Case Study Research: Design and Methods. Thousand Oaks, California: Sage Publications.

Yin, R.K. (2003). Case Study Research. Thousand Oaks, California: Sage Publications. 


\section{Appendices}

\section{Appendix 1: Descriptive analysis}

The summary of valid and complete returned surveys

\begin{tabular}{|c|c|c|c|c|c|c|c|c|c|c|c|c|c|c|c|c|c|c|c|}
\hline \multirow[t]{2}{*}{ No. } & \multirow[t]{2}{*}{ Regional offices } & \multicolumn{3}{|c|}{ Researchers } & \multicolumn{3}{|c|}{ Research assistants } & \multicolumn{3}{|c|}{ Managers } & \multicolumn{3}{|c|}{ General employees } & \multirow[t]{2}{*}{ TPS } & \multicolumn{2}{|c|}{ TR } & \multirow[t]{2}{*}{ TRS } & \multicolumn{2}{|c|}{$\begin{array}{l}\text { TOTAL number of } \\
\text { returned surveys }\end{array}$} \\
\hline & & PS & PR & OR & PS & PR & OR & PS & PR & OR & PS & PR & OR & & TOR & TPR & & $\mathrm{I}$ & $\mathrm{c}$ \\
\hline 1 & $\begin{array}{l}\text { Secretariat of FORDA, } \\
\text { JAKARTA }\end{array}$ & 0 & 0 & 0 & 0 & 0 & 0 & 10 & 6 & 2 & 25 & 27 & 1 & 35 & 3 & 33 & 36 & 9 & 27 \\
\hline 2 & CFNCRD, BOGOR & 15 & 3 & 1 & 15 & 4 & 1 & 5 & 4 & 0 & 20 & 11 & 2 & 55 & 4 & 22 & 26 & 10 & 16 \\
\hline 3 & CFPRD, BOGOR & 15 & 0 & 0 & 15 & 0 & 0 & 5 & 0 & 0 & 15 & 0 & 0 & 50 & 0 & 0 & 0 & 0 & 0 \\
\hline 4 & CSEFPR ,BOGOR & 10 & 1 & 0 & 10 & 0 & 0 & 5 & 3 & 0 & 10 & 8 & 1 & 35 & 1 & 12 & 13 & 4 & 9 \\
\hline 5 & CPFRD, BOGOR & 15 & 11 & 0 & 5 & 3 & 0 & 5 & 0 & 2 & 10 & 7 & 1 & 35 & 3 & 21 & 24 & 14 & 10 \\
\hline 6 & CDR, SAMARINDA & 10 & 7 & 0 & 10 & 4 & 0 & 5 & 4 & 0 & 10 & 7 & 0 & 35 & 0 & 22 & 22 & 10 & 12 \\
\hline 7 & CBFTIR, YOGYAKARTA & 10 & 5 & 2 & 10 & 4 & 0 & 5 & 6 & 1 & 15 & 1 & 5 & 40 & 8 & 16 & 24 & 5 & 19 \\
\hline 8 & FRI AEK NAULI & 10 & 11 & 0 & 10 & 9 & 0 & 3 & 2 & 0 & 15 & 4 & 0 & 38 & 0 & 26 & 26 & 7 & 19 \\
\hline 9 & FRI MAKASSAR & 10 & 10 & 0 & 10 & 10 & 0 & 3 & 3 & 0 & 15 & 14 & 0 & 38 & 0 & 37 & 37 & 10 & 27 \\
\hline 10 & FRI KUPANG & 10 & 10 & 1 & 5 & 6 & 0 & 2 & 2 & 0 & 10 & 9 & 0 & 27 & 1 & 27 & 28 & 9 & 19 \\
\hline 11 & FRI MANOKWARI & 10 & 7 & 0 & 10 & 4 & 0 & 2 & 2 & 1 & 10 & 8 & 0 & 32 & 1 & 21 & 22 & 7 & 15 \\
\hline 12 & FRI PALEMBANG & 10 & 12 & 0 & 5 & 0 & 0 & 3 & 2 & 0 & 15 & 12 & 0 & 33 & 0 & 26 & 26 & 7 & 19 \\
\hline 13 & FRI BANJARBARU & 10 & 9 & 1 & 10 & 9 & 1 & 3 & 3 & 0 & 15 & 13 & 2 & 38 & 4 & 34 & 38 & 14 & 24 \\
\hline 14 & FRI MATARAM & 10 & 8 & 0 & 5 & 7 & 0 & 2 & 2 & 0 & 3 & 3 & 0 & 20 & 0 & 20 & 20 & 7 & 13 \\
\hline 15 & FRI MANADO & 5 & 3 & 0 & 5 & 2 & 0 & 3 & 3 & 0 & 3 & 1 & 0 & 16 & 0 & 9 & 9 & 3 & 6 \\
\hline 16 & FRI SOLO & 10 & 4 & 4 & 10 & 6 & 0 & 3 & 2 & 2 & 10 & 4 & 1 & 33 & 7 & 16 & 23 & 9 & 14 \\
\hline 17 & FRI CIAMIS & 10 & 0 & 7 & 10 & 0 & 1 & 2 & 0 & 2 & 10 & 0 & 3 & 32 & 13 & 0 & 13 & 2 & 11 \\
\hline 18 & FBTRI BOGOR & 10 & 1 & 0 & 10 & 2 & 0 & 2 & 1 & 0 & 10 & 4 & 0 & 32 & 0 & 8 & 8 & 4 & 4 \\
\hline 19 & FBTRI SAMBOJA & 8 & 2 & 0 & 8 & 5 & 0 & 2 & 0 & 0 & 5 & 3 & 1 & 23 & 1 & 10 & 11 & 2 & 9 \\
\hline 20 & PFFPRI KUOK & 10 & 7 & 0 & 10 & 4 & 0 & 2 & 2 & 0 & 3 & 3 & 0 & 25 & 0 & 16 & 16 & 1 & 15 \\
\hline & TOTAL SAMPLE & 198 & 111 & 16 & 173 & 79 & 3 & 72 & 47 & 10 & 229 & 139 & 17 & 672 & 46 & 376 & 422 & 134 & 288 \\
\hline
\end{tabular}

$P S=$ Paper-based survey sent for each stralum

$P R=$ Paper-based survey returned for each stratum

$\mathrm{OR}=$ Online survey returned for each stratum

TPS = TOTAL number of paper-based survey sent

TOR = TOTAL number of online survey returned

TPR = TOTAL number of paper-based survey returned

TRS = TOTAL number of surveys returned (Online + Paper-based)

$I=$ Incomplete returned surveys

$\mathrm{C}=$ Complete returned surveys which were used for data analysis in this research 


\section{Appendix 2: First reliability test}

\section{TOTAL I - Technological skills readiness - inter-item correlation}

\begin{tabular}{|c|c|c|c|}
\hline & & $\mathrm{N}$ & $\%$ \\
\hline \multirow[t]{3}{*}{ Cases } & Valid & 288 & 100 \\
\hline & Excludeda & 0 & 0 \\
\hline & Total & 288 & 100 \\
\hline
\end{tabular}

Reliability Statistics

\begin{tabular}{|c|c|c|}
\hline Cronbach's Alpha & $\begin{array}{c}\text { Cronbach's Alpha Based } \\
\text { on Standardised Items }\end{array}$ & No. of Items \\
\hline .927 & .928 & 6 \\
\hline
\end{tabular}

Item Statistics

\begin{tabular}{|c|c|c|c|}
\hline & Mean & Std. Deviation & $\mathrm{N}$ \\
\hline $\mathrm{Cl} 1$ & 3.96 & .983 & 288 \\
$\mathrm{Cl} 2$ & 3.42 & 1.072 & 288 \\
$\mathrm{Cl} 3$ & 2.97 & 1.162 & 288 \\
$\mathrm{Cl} 4$ & 3.30 & 1.158 & 288 \\
$\mathrm{Cl} 5$ & 3.62 & 1.215 & 288 \\
$\mathrm{Cl} 6$ & 2.42 & .887 & 288 \\
\hline
\end{tabular}

Inter-Item Correlation Matrix

\begin{tabular}{|r|r|r|r|r|r|r|}
\hline & \multicolumn{1}{c|}{$\mathrm{Cl} 1$} & \multicolumn{1}{c|}{$\mathrm{Cl} 2$} & \multicolumn{1}{c|}{$\mathrm{Cl} 3$} & \multicolumn{1}{c|}{$\mathrm{Cl} 4$} & \multicolumn{1}{c|}{$\mathrm{Cl} 5$} & \multicolumn{1}{c|}{$\mathrm{Cl} 6$} \\
\hline $\mathrm{Cl} 1$ & 1.000 & .773 & .612 & .727 & .768 & .519 \\
$\mathrm{Cl} 2$ & .773 & 1.000 & .722 & .763 & .718 & .579 \\
$\mathrm{Cl} 3$ & .612 & .722 & 1.000 & .710 & .644 & .660 \\
$\mathrm{Cl} 4$ & .727 & .763 & .710 & 1.000 & .805 & .664 \\
$\mathrm{Cl} 5$ & .768 & .718 & .644 & .805 & 1.000 & .570 \\
$\mathrm{Cl} 6$ & .519 & .579 & .660 & .664 & .570 & 1.000 \\
\hline
\end{tabular}

Summary Item Statistics

\begin{tabular}{|l|c|c|c|c|c|c|c|}
\hline & Mean & Minimum & Maximum & Range & $\begin{array}{c}\text { Maximum / } \\
\text { Minimum }\end{array}$ & Variance & No. of Items \\
\hline Item Means & 3.281 & 2.417 & 3.958 & 1.542 & 1.638 & .287 & 6 \\
Inter-Item Correlations & .682 & .519 & .805 & .286 & 1.550 & .007 & 6 \\
\hline
\end{tabular}

\begin{tabular}{|c|c|c|c|c|c|}
\hline & $\begin{array}{l}\text { Scale Mean if } \\
\text { Item Deleted }\end{array}$ & $\begin{array}{l}\text { Scale Variance } \\
\text { if Item Deleted }\end{array}$ & $\begin{array}{c}\text { Corrected Item- } \\
\text { Total } \\
\text { Correlation }\end{array}$ & $\begin{array}{l}\text { Squared Multiple } \\
\text { Correlation }\end{array}$ & $\begin{array}{l}\text { Cronbach's } \\
\text { Alpha if Item } \\
\text { Deleted }\end{array}$ \\
\hline $\mathrm{Cl} 1$ & 15.73 & 22.721 & .792 & .694 & .914 \\
\hline $\mathrm{Cl} 2$ & 16.27 & 21.669 & .831 & .722 & .908 \\
\hline $\mathrm{Cl} 3$ & 16.72 & 21.473 & .770 & .629 & .917 \\
\hline $\mathrm{Cl} 4$ & 16.39 & 20.684 & .863 & .758 & .904 \\
\hline $\mathrm{Cl} 5$ & 16.07 & 20.619 & .817 & .722 & .911 \\
\hline $\mathrm{Cl} 6$ & 17.27 & 24.365 & .681 & .513 & .928 \\
\hline
\end{tabular}

Scale Statistics

\begin{tabular}{|c|c|c|c|}
\hline Mean & Variance & Std. Deviation & No. of Items \\
\hline 19.69 & 31.115 & 5.578 & 6 \\
\hline
\end{tabular}




\section{TOTAL II - Online learning style - inter-item correlation}

Case Processing Summary

\begin{tabular}{|ll|c|c|}
\hline & $\mathrm{N}$ & $\%$ \\
\hline Cases & Valid & 288 & 100 \\
& Excludeda & 0 & 0 \\
& Total & 288 & 100 \\
\hline
\end{tabular}

a. Listwise deletion based on all variables in the procedure.

\begin{tabular}{|c|c|c|}
\hline \multicolumn{3}{|c|}{ Reliability Statistics } \\
\hline Cronbach's Alpha & $\begin{array}{c}\text { Cronbach's Alpha Based } \\
\text { on Standardised Items }\end{array}$ & No. of Items \\
\hline .576 & .562 & 9 \\
\hline
\end{tabular}

Item Statistics

\begin{tabular}{|l|c|c|c|}
\hline & Mean & Std. Deviation & $\mathrm{N}$ \\
\hline CII1 & 4.09 & .732 & 288 \\
CII2 & 3.70 & .765 & 288 \\
CII3 & 3.72 & .746 & 288 \\
CII4 & 3.77 & .757 & 288 \\
CII5 & 2.78 & .859 & 288 \\
CII6 & 3.30 & .857 & 288 \\
CII7 & 2.12 & .756 & 288 \\
CII8 & 1.98 & .720 & 288 \\
CII9 & 4.14 & .597 & 288 \\
\hline
\end{tabular}

Inter-Item Correlation Matrix

\begin{tabular}{|c|c|c|c|c|c|c|c|c|c|}
\hline & Cll1 & $\mathrm{Cll} 2$ & Cll3 & Cll4 & Cll5 & CII6 & $\mathrm{Cll} 7$ & Cll8 & Cll9 \\
\hline Cll1 & 1.000 & .602 & .410 & .515 & .076 & .256 & .000 & -.057 & .131 \\
\hline CII2 & .602 & 1.000 & .454 & .547 & .080 & .291 & .012 & -.047 & .151 \\
\hline Cll3 & .410 & .454 & 1.000 & .475 & -.015 & .137 & -.047 & -.061 & .102 \\
\hline $\mathrm{Cll} 4$ & .515 & .547 & .475 & 1.000 & .024 & .267 & .028 & -.084 & .162 \\
\hline Cll5 & .076 & .080 & -.015 & .024 & 1.000 & .054 & .244 & .101 & -.041 \\
\hline Cll6 & .256 & .291 & .137 & .267 & .054 & 1.000 & .090 & .042 & .006 \\
\hline CII7 & .000 & .012 & -.047 & .028 & .244 & .090 & 1.000 & .522 & -.445 \\
\hline Cll8 & -.057 & -.047 & -.061 & -.084 & .101 & .042 & .522 & 1.000 & -.496 \\
\hline ClI9 & .131 & .151 & .102 & .162 & -.041 & .006 & -.445 & -.496 & 1.000 \\
\hline
\end{tabular}

Summary Item Statistics

\begin{tabular}{|l|c|c|c|c|c|c|c|}
\hline & Mean & Minimum & Maximum & Range & $\begin{array}{c}\text { Maximum / } \\
\text { Minimum }\end{array}$ & Variance & No. of Items \\
\hline Item Means & 3.290 & 1.983 & 4.139 & 2.156 & 2.088 & .662 & 9 \\
Inter-Item Correlations & .125 & -.496 & .602 & 1.098 & -1.213 & .060 & 9 \\
\hline
\end{tabular}


Item-Total Statistics

\begin{tabular}{|l|c|c|c|c|c|}
\hline & $\begin{array}{c}\text { Scale Mean if } \\
\text { Item Deleted }\end{array}$ & $\begin{array}{c}\text { Scale Variance } \\
\text { if Item Deleted }\end{array}$ & $\begin{array}{c}\text { Corrected Item- } \\
\text { Total } \\
\text { Correlation }\end{array}$ & $\begin{array}{c}\text { Squared } \\
\text { Multiple } \\
\text { Correlation }\end{array}$ & $\begin{array}{c}\text { Cronbach's } \\
\text { Alpha if Item } \\
\text { Deleted }\end{array}$ \\
\hline CII1 & 25.52 & 7.895 & .526 & .425 & .472 \\
CII2 & 25.91 & 7.597 & .572 & .473 & .453 \\
CII3 & 25.89 & 8.406 & .377 & .296 & .516 \\
CII4 & 25.84 & 7.816 & .521 & .419 & .471 \\
CII5 & 26.83 & 9.164 & .133 & .075 & .590 \\
CII6 & 26.31 & 8.382 & .298 & .115 & .538 \\
CII7 & 27.49 & 9.429 & .128 & .370 & .586 \\
CII8 & 27.63 & 10.025 & .011 & .362 & .613 \\
CII9 & 25.47 & 10.612 & -.096 & .322 & .625 \\
\hline
\end{tabular}

Scale Statistics

\begin{tabular}{|c|c|c|c|}
\hline Mean & Variance & Std. Deviation & No. of Items \\
\hline 29.61 & 10.594 & 3.255 & 9 \\
\hline
\end{tabular}

TOTAL III - Equipment/infrastructure readiness - inter-item correlation

\begin{tabular}{|c|c|c|c|}
\hline \multicolumn{4}{|c|}{ Case Processing Summary } \\
\hline & & $\mathrm{N}$ & $\%$ \\
\hline \multirow[t]{3}{*}{ Cases } & Valid & 288 & 100 \\
\hline & Excludeda & 0 & 0 \\
\hline & Total & 288 & 100 \\
\hline
\end{tabular}

a. Listwise deletion based on all variables in the procedure.

Reliability Statistics

\begin{tabular}{|c|c|c|}
\hline Cronbach's Alpha & $\begin{array}{c}\text { Cronbach's Alpha Based } \\
\text { on Standardised Items }\end{array}$ & No. of Items \\
\hline .774 & .793 & 8 \\
\hline
\end{tabular}

Item Statistics

\begin{tabular}{|c|c|c|c|}
\hline \multicolumn{4}{|c|}{ Item Statistics } \\
\hline & Mean & Std. Deviation & $\mathrm{N}$ \\
\hline CIII1 & 4.23 & 1.203 & 288 \\
CII2 & 3.83 & 1.423 & 288 \\
CIII3 & 4.13 & 1.251 & 288 \\
CIII4 & 3.38 & 1.422 & 288 \\
CIII5 & 2.88 & 1.516 & 288 \\
CIII6 & 4.38 & .646 & 288 \\
CIII7 & 4.23 & .631 & 288 \\
CIII8 & 4.32 & .626 & 288 \\
\hline
\end{tabular}

Inter-Item Correlation Matrix

\begin{tabular}{|l|r|r|r|r|r|r|r|r|}
\hline & \multicolumn{1}{|c|}{ CIII1 } & \multicolumn{1}{c|}{ CIII2 } & \multicolumn{1}{c|}{ CIII3 } & \multicolumn{1}{c|}{ CIII4 } & \multicolumn{1}{c|}{ CIII5 } & \multicolumn{1}{c|}{ CIII6 } & \multicolumn{1}{c|}{ CIII7 } & \multicolumn{1}{c|}{ CIII8 } \\
\hline CIII1 & 1.000 & .632 & .637 & .353 & .211 & .294 & .224 & .285 \\
CIII2 & .632 & 1.000 & .698 & .391 & .200 & .295 & .214 & .246 \\
CIII3 & .637 & .698 & 1.000 & .387 & .172 & .335 & .276 & .271 \\
CIII4 & .353 & .391 & .387 & 1.000 & .646 & .111 & .179 & .132 \\
CIII5 & .211 & .200 & .172 & .646 & 1.000 & -.005 & .030 & .013 \\
CIII6 & .294 & .295 & .335 & .111 & -.005 & 1.000 & .738 & .561 \\
CIII7 & .224 & .214 & .276 & .179 & .030 & .738 & 1.000 & .548 \\
CIII8 & .285 & .246 & .271 & .132 & .013 & .561 & .548 & 1.000 \\
\hline
\end{tabular}


Summary Item Statistics

\begin{tabular}{|l|c|c|c|c|c|c|c|}
\hline & Mean & Minimum & Maximum & Range & $\begin{array}{c}\text { Maximum / } \\
\text { Minimum }\end{array}$ & Variance & No. of Items \\
\hline Item Means & 3.921 & 2.875 & 4.378 & 1.503 & 1.523 & .285 & 8 \\
Inter-Item Correlations & .324 & -.005 & .738 & .743 & -150.916 & .044 & 8 \\
\hline
\end{tabular}

Item-Total Statistics

\begin{tabular}{|l|c|c|c|c|c|}
\hline & $\begin{array}{c}\text { Scale Mean if } \\
\text { Item Deleted }\end{array}$ & $\begin{array}{c}\text { Scale Variance if } \\
\text { Item Deleted }\end{array}$ & $\begin{array}{c}\text { Corrected Item- } \\
\text { Total Correlation }\end{array}$ & $\begin{array}{c}\text { Squared Multiple } \\
\text { Correlation }\end{array}$ & $\begin{array}{c}\text { Cronbach's Alpha } \\
\text { if Item Deleted }\end{array}$ \\
\hline CIII1 & 27.14 & 23.953 & .619 & .491 & .723 \\
CIII2 & 27.55 & 22.200 & .631 & .559 & .718 \\
CIII3 & 27.24 & 23.291 & .649 & .573 & .716 \\
CIII4 & 27.99 & 22.951 & .566 & .520 & .732 \\
CIII5 & 28.50 & 25.247 & .338 & .433 & .785 \\
CIII6 & 26.99 & 29.477 & .398 & .603 & .765 \\
CIII7 & 27.15 & 29.714 & .374 & .584 & .768 \\
CIII8 & 27.05 & 29.854 & .357 & .371 & .769 \\
\hline
\end{tabular}

Scale Statistics

\begin{tabular}{|c|c|c|c|}
\hline Mean & Variance & Std. Deviation & No. of Items \\
\hline 31.37 & 32.687 & 5.717 & 8 \\
\hline
\end{tabular}


TOTAL IV - Attitude readiness - inter-item correlation

Case Processing Summary

\begin{tabular}{|c|c|c|c|}
\hline & & $\mathrm{N}$ & $\%$ \\
\hline \multirow[t]{3}{*}{ Cases } & Valid & 288 & 100 \\
\hline & Excludeda & 0 & 0 \\
\hline & Total & 288 & 100 \\
\hline
\end{tabular}

a. Listwise deletion based on all variables in the procedure.

\begin{tabular}{|c|c|c|}
\hline \multicolumn{3}{|c|}{ Reliability Statistics } \\
\hline Cronbach's Alpha & $\begin{array}{c}\text { Cronbach's Alpha Based } \\
\text { on Standardised Items }\end{array}$ & No. of Items \\
\hline .899 & .907 & 23 \\
\hline
\end{tabular}

\begin{tabular}{|l|c|c|c|}
\hline \multicolumn{4}{|c|}{ Item Statistics } \\
\hline & Mean & Std. Deviation & $\mathrm{N}$ \\
\hline CIV1 & 4.01 & .691 & 288 \\
CIV2 & 4.06 & .701 & 288 \\
CIV3 & 3.00 & .905 & 288 \\
CIV4 & 4.26 & .644 & 288 \\
CIV5 & 3.89 & .872 & 288 \\
CIV6 & 4.24 & .613 & 288 \\
CIV7 & 4.01 & .783 & 288 \\
CIV8 & 3.95 & .748 & 288 \\
CIV9 & 2.98 & .901 & 288 \\
CIV10 & 3.90 & .681 & 288 \\
CIV11 & 3.93 & .685 & 288 \\
CIV12 & 4.44 & .563 & 288 \\
CIV13 & 4.25 & .595 & 288 \\
CIV14 & 3.62 & .723 & 288 \\
CIV15 & 3.40 & .853 & 288 \\
CIV16 & 3.30 & .853 & 288 \\
CIV17 & 4.06 & .644 & 288 \\
CIV18 & 4.31 & .599 & 288 \\
CIV19 & 4.22 & .651 & 288 \\
CIV20 & 4.13 & .599 & 288 \\
CIV21 & 3.98 & .668 & 288 \\
CIV22 & 3.79 & .692 & 288 \\
CIV23 & 3.82 & .774 & 288 \\
\hline
\end{tabular}


Inter-Item Correlation Matrix

\begin{tabular}{|c|c|c|c|c|c|c|c|c|c|c|c|c|c|c|c|c|c|c|c|c|c|c|c|}
\hline & IV1 & $\mathrm{CIV}$ & CIV3 & CIV4 & CIV5 & CIV6 & CIV7 & CIV8 & $\mathrm{CIV}$ & CIV10 & CIV11 & CIV12 & CIV13 & CIV14 & CIV15 & CIV16 & CIV17 & CIV18 & CIV19 & CIV20 & CIV21 & CIV22 & CIV23 \\
\hline CIV1 & 1.000 & .351 & .346 & .307 & .164 & .290 & .373 & .372 & .286 & .328 & .303 & .329 & .299 & .196 & .164 & .219 & .335 & .329 & .382 & .333 & .348 & .340 & .323 \\
\hline CIV2 & .351 & 1.000 & .285 & .292 & .375 & .302 & .310 & .450 & .212 & .347 & .393 & .264 & .310 & .207 & .173 & .135 & .263 & .225 & 309 & .223 & .345 & 491 & .448 \\
\hline CIV3 & .346 & .285 & 1.000 & .160 & .031 & .124 & .266 & 247 & .325 & .283 & .220 & .147 & .102 & .236 & .156 & .170 & .167 & .062 & .141 & .147 & .237 & .196 & .185 \\
\hline CIV4 & .307 & .292 & .160 & 1.000 & .144 & .525 & .342 & .321 & .101 & .383 & .436 & .446 & .353 & .144 & .068 & .055 & .427 & .437 & .378 & .336 & .296 & .207 & .203 \\
\hline CIV5 & .164 & .375 & .031 & .144 & 1.000 & .140 & .164 & .137 & -.039 & .152 & .092 & .121 & .295 & .076 & .022 & -.006 & .191 & .205 & .148 & 101 & .086 & .389 & .384 \\
\hline CIV6 & .290 & .302 & .124 & .525 & .140 & 1.000 & .410 & .403 & .193 & .405 & .412 & .484 & .451 & .149 & .120 & .090 & .425 & .495 & .479 & .456 & .463 & .272 & .309 \\
\hline CIV7 & .373 & .310 & .266 & .342 & .164 & .410 & 1.000 & .620 & .331 & .530 & .527 & |451 & .370 & .294 & 163 & .127 & .400 & .411 & .427 & .429 & .514 & .382 & .364 \\
\hline CIV8 & .372 & .450 & .247 & .321 & .137 & .403 & .620 & 1.000 & .402 & .552 & .552 & .395 & .284 & .136 & .110 & .153 & .403 & .357 & .450 & .402 & .487 & .352 & .341 \\
\hline CIV9 & .286 & .212 & .325 & .101 & -.039 & .193 & .331 & .402 & 1.000 & .359 & .257 & .248 & .187 & .221 & .144 & .091 & .231 & .201 & .259 & .251 & .422 & .238 & .204 \\
\hline CIV10 & .328 & .347 & .283 & .383 & .152 & .405 & .530 & .552 & .359 & 1.000 & .560 & .385 & .326 & .165 & .090 & .117 & .425 & .397 & .410 & 399 & .501 & .252 & .28 \\
\hline CIV11 & .303 & .393 & .220 & .436 & .092 & .412 & .527 & .552 & .257 & .560 & 1.000 & .432 & .342 & .178 & 161 & .096 & .435 & .374 & .472 & .405 & .469 & .388 & .351 \\
\hline CIV12 & .329 & .264 & 147 & .446 & .121 & .484 & .451 & .395 & .248 & .385 & .432 & 1.000 & .538 & .227 & .143 & .106 & .461 & .559 & .454 & .395 & .395 & .263 & .252 \\
\hline CIV13 & .299 & .310 & .102 & .353 & .295 & .451 & .370 & 284 & .187 & .326 & .342 & .538 & 1.000 & .277 & .178. & .141 & .410 & .453 & 398 & .388 & .347 & .371 & .307 \\
\hline CIV14 & .196 & .207 & .236 & .144 & .076 & .149 & .294 & .136 & .221 & .165 & .178 & .227 & 277 & 1.000 & .478 & .323 & .270 & .158 & .233 & .237 & .243 & .209 & .240 \\
\hline CIV15 & .164 & .173 & .156 & .068 & .022 & .120 & .163 & 110 & .144 & .090 & .161 & 143 & .178 & .478 & 1.000 & .616 & .277 & .097 & 186 & 109 & .204 & .075 & 154 \\
\hline CIV16 & .219 & .135 & .170 & .055 & -.006 & .090 & .127 & .153 & .091 & .117 & .096 & .106 & .141 & .323 & .616 & 1.000 & .299 & .112 & .217 & .201 & .189 & .030 & .097 \\
\hline CIV17 & .335 & .263 & .167 & .427 & .191 & .425 & .400 & .403 & .231 & .425 & .435 & .461 & .410 & .270 & .277 & .299 & 1.000 & .524 & .568 & 478 & .489 & .307 & .243 \\
\hline CIV18 & .329 & .225 & .062 & .437 & 205 & .495 & .411 & 357 & .201 & 397 & .374 & .559 & .453 & .158 & .097 & .112 & .524 & 1.000 & .655 & .460 & .425 & 280 & .275 \\
\hline CIV19 & .382 & .309 & .141 & .378 & .148 & .479 & .427 & .450 & .259 & .410 & .472 & .454 & .398 & .233 & .186 & .217 & .568 & .655 & 1.000 & .559 & .516 & .289 & .279 \\
\hline CIV20 & .333 & .223 & .147 & .336 & 101 & .456 & .429 & .402 & .251 & 399 & .405 & 395 & .388 & .237 & 109 & .201 & .478 & .460 & .559 & 1.000 & .599 & .285 & .321 \\
\hline CIV21 & .348 & .345 & .237 & .296 & .086 & .463 & .514 & .487 & .422 & .501 & .469 & .395 & .347 & .243 & 204 & .189 & .489 & .425 & .516 & .599 & 1.000 & .390 & .337 \\
\hline CIV22 & .340 & .491 & .196 & .207 & .389 & .272 & .382 & .352 & .238 & .252 & .388 & .263 & .371 & .209 & .075 & .030 & .307 & .280 & 289 & 285 & .390 & 1.000 & .614 \\
\hline CIV23 & .323 & .448 & 185 & .203 & .384 & .309 & .364 & 341 & 204 & 284 & .351 & .252 & .307 & .240 & .154 & .097 & .243 & .275 & .279 & 321 & .337 & 614 & 1.00 \\
\hline
\end{tabular}


Summary Item Statistics

\begin{tabular}{|l|c|c|c|c|c|c|c|}
\hline & Mean & Minimum & Maximum & Range & $\begin{array}{c}\text { Maximum / } \\
\text { Minimum }\end{array}$ & Variance & No. of Items \\
\hline Item Means & 3.893 & 2.976 & 4.441 & 1.465 & 1.492 & .156 & 23 \\
Inter-Item Correlations & .299 & -.039 & .655 & .694 & -16.839 & .019 & 23 \\
\hline
\end{tabular}

Item-Total Statistics

\begin{tabular}{|c|c|c|c|c|c|}
\hline & $\begin{array}{l}\text { Scale Mean if } \\
\text { Item Deleted }\end{array}$ & $\begin{array}{l}\text { Scale Variance } \\
\text { if Item Deleted }\end{array}$ & $\begin{array}{l}\text { Corrected Item- } \\
\text { Total Correlation }\end{array}$ & $\begin{array}{l}\text { Squared Multiple } \\
\text { Correlation }\end{array}$ & $\begin{array}{l}\text { Cronbach's Alpha if } \\
\text { Item Deleted }\end{array}$ \\
\hline CIV1 & 85.52 & 78.431 & .536 & .334 & .894 \\
\hline CIV2 & 85.48 & 78.278 & .540 & .449 & .894 \\
\hline CIV3 & 86.53 & 79.170 & .340 & .250 & .900 \\
\hline CIV4 & 85.28 & 79.491 & .485 & .417 & .895 \\
\hline CIV5 & 85.65 & 80.724 & .254 & .318 & .902 \\
\hline CIV6 & 85.30 & 78.886 & .570 & .473 & .893 \\
\hline CIV7 & 85.53 & 75.964 & .651 & .541 & .891 \\
\hline CIV8 & 85.58 & 76.649 & .631 & .568 & .891 \\
\hline CIV9 & 86.56 & 78.220 & .404 & .322 & .898 \\
\hline CIV10 & 85.63 & 77.725 & .606 & .499 & .892 \\
\hline CIV11 & 85.60 & 77.550 & .618 & .525 & .892 \\
\hline CIV12 & 85.09 & 79.333 & .580 & .492 & .894 \\
\hline CIV13 & 85.29 & 79.251 & .554 & .436 & .894 \\
\hline CIV14 & 85.92 & 79.826 & .396 & .343 & .897 \\
\hline CIV15 & 86.14 & 79.946 & .314 & .500 & .900 \\
\hline CIV16 & 86.23 & 80.353 & .287 & .443 & .901 \\
\hline CIV17 & 85.48 & 77.881 & .631 & .501 & .892 \\
\hline CIV18 & 85.23 & 78.986 & .575 & .570 & .893 \\
\hline CIV19 & 85.31 & 77.700 & .640 & .587 & .892 \\
\hline CIV2O & 85.40 & 78.883 & .585 & .502 & .893 \\
\hline CIV21 & 85.56 & 77.293 & .659 & .560 & .891 \\
\hline CIV22 & 85.74 & 78.512 & .528 & .523 & .894 \\
\hline CIV23 & 85.71 & 77.788 & .519 & .468 & .894 \\
\hline
\end{tabular}

Scale Statistics

\begin{tabular}{|c|c|c|c|}
\hline Mean & Variance & Std. Deviation & No. of Items \\
\hline 89.53 & 85.469 & 9.245 & 23 \\
\hline
\end{tabular}

\section{TOTAL V - Human resource readiness - inter-item correlation}

\begin{tabular}{|c|c|c|c|}
\hline & & $\mathrm{N}$ & $\%$ \\
\hline \multirow[t]{3}{*}{ Cases } & Valid & 288 & 100 \\
\hline & Excludeda & 0 & 0 \\
\hline & Total & 288 & 100 \\
\hline
\end{tabular}

a. Listwise deletion based on all variables in the procedure.

Reliability Statistics

\begin{tabular}{|c|c|c|}
\hline Cronbach's Alpha & $\begin{array}{c}\text { Cronbach's Alpha Based } \\
\text { on Standardised Items }\end{array}$ & No. of Items \\
\hline .865 & .863 & 11 \\
\hline
\end{tabular}


Item Statistics

\begin{tabular}{|l|c|c|c|}
\hline & Mean & Std. Deviation & $\mathrm{N}$ \\
\hline CV1 & 3.63 & .803 & 288 \\
CV2 & 3.73 & .692 & 288 \\
CV3 & 3.68 & .725 & 288 \\
CV4 & 3.70 & .827 & 288 \\
CV5 & 2.48 & .910 & 288 \\
CV6 & 2.45 & .905 & 288 \\
CV7 & 2.46 & .914 & 288 \\
CV8 & 1.75 & .755 & 288 \\
CV9 & 2.00 & .847 & 288 \\
CV10 & 1.88 & .804 & 288 \\
CV11 & 1.61 & .752 & 288 \\
\hline
\end{tabular}

Inter-Item Correlation Matrix

\begin{tabular}{|l|r|r|r|r|r|r|r|r|r|r|r|}
\hline & \multicolumn{1}{|c|}{ CV1 } & \multicolumn{1}{c|}{ CV2 } & \multicolumn{1}{c|}{ CV3 } & \multicolumn{1}{c|}{ CV4 } & \multicolumn{1}{c|}{ CV5 } & \multicolumn{1}{c|}{ CV6 } & \multicolumn{1}{c|}{ CV7 } & CV8 & CV9 & CV10 & CV11 \\
\hline CV1 & 1.000 & .618 & .433 & .498 & .214 & .206 & .083 & .182 & .164 & .246 & .212 \\
CV2 & .618 & 1.000 & .587 & .465 & .180 & .186 & .135 & .150 & .137 & .245 & .218 \\
CV3 & .433 & .587 & 1.000 & .507 & .091 & .126 & .173 & .070 & .062 & .134 & .059 \\
CV4 & .498 & .465 & .507 & 1.000 & .291 & .296 & .303 & .233 & .179 & .221 & .184 \\
CV5 & .214 & .180 & .091 & .291 & 1.000 & .705 & .585 & .465 & .492 & .562 & .478 \\
CV6 & .206 & .186 & .126 & .296 & .705 & 1.000 & .672 & .489 & .536 & .556 & .444 \\
CV7 & .083 & .135 & .173 & .303 & .585 & .672 & 1.000 & .555 & .549 & .520 & .417 \\
CV8 & .182 & .150 & .070 & .233 & .465 & .489 & .555 & 1.000 & .670 & .713 & .643 \\
CV9 & .164 & .137 & .062 & .179 & .492 & .536 & .549 & .670 & 1.000 & .691 & .558 \\
CV10 & .246 & .245 & .134 & .221 & .562 & .556 & .520 & .713 & .691 & 1.000 & .669 \\
CV11 & .212 & .218 & .059 & .184 & .478 & .444 & .417 & .643 & .558 & .669 & 1.000 \\
\hline
\end{tabular}

Summary Item Statistics

\begin{tabular}{|l|c|c|c|c|c|c|c|}
\hline & Mean & Minimum & Maximum & Range & Maximum / Minimum & Variance & No. of Items \\
\hline Item Means & 2.669 & 1.615 & 3.726 & 2.111 & 2.308 & .728 & 11 \\
Inter-Item Correlations & .365 & .059 & .713 & .654 & 12.182 & .043 & 11 \\
\hline
\end{tabular}

Item-Total Statistics

\begin{tabular}{|l|c|c|c|c|c|}
\hline & $\begin{array}{c}\text { Scale Mean if } \\
\text { Item Deleted }\end{array}$ & $\begin{array}{c}\text { Scale Variance } \\
\text { if Item Deleted }\end{array}$ & $\begin{array}{c}\text { Corrected Item- } \\
\text { Total Correlation }\end{array}$ & $\begin{array}{c}\text { Squared Multiple } \\
\text { Correlation }\end{array}$ & $\begin{array}{c}\text { Cronbach's Alpha } \\
\text { if Item Deleted }\end{array}$ \\
\hline CV1 & 25.73 & 30.029 & .409 & .467 & .864 \\
CV2 & 25.64 & 30.546 & .426 & .524 & .862 \\
CV3 & 25.68 & 31.165 & .320 & .439 & .869 \\
CV4 & 25.66 & 29.389 & .469 & .416 & .860 \\
CV5 & 26.89 & 27.342 & .642 & .565 & .847 \\
CV6 & 26.91 & 27.146 & .669 & .618 & .845 \\
CV7 & 26.90 & 27.429 & .629 & .574 & .849 \\
CV8 & 27.61 & 28.441 & .655 & .628 & .848 \\
CV9 & 27.36 & 27.911 & .631 & .575 & .848 \\
CV10 & 27.49 & 27.582 & .717 & .671 & .842 \\
CV11 & 27.75 & 28.859 & .601 & .524 & .851 \\
\hline
\end{tabular}

Scale Statistics

\begin{tabular}{|c|c|c|c|}
\hline Mean & Variance & Std. Deviation & No. of Items \\
\hline 29.36 & 34.280 & 5.855 & 11 \\
\hline
\end{tabular}




\section{TOTAL VI - Environmental - inter-item correlation}

\begin{tabular}{|c|c|c|c|}
\hline \multicolumn{4}{|c|}{ Case Processing Summary } \\
\hline & & $\mathrm{N}$ & $\%$ \\
\hline \multirow{3}{*}{ Cases } & Valid & 288 & 100 \\
\hline & Excludeda & 0 & 0 \\
\hline & Total & 288 & 100 \\
\hline
\end{tabular}

a. Listwise deletion based on all variables in the procedure.

Reliability Statistics

\begin{tabular}{|c|c|c|}
\hline Cronbach's Alpha & $\begin{array}{c}\text { Cronbach's Alpha Based } \\
\text { on Standardised Items }\end{array}$ & No. of Items \\
\hline .063 & .074 & 4 \\
\hline
\end{tabular}

\begin{tabular}{|l|c|c|c|}
\multicolumn{4}{|c|}{ Item Statistics } \\
\hline & Mean & Std. Deviation & $\mathrm{N}$ \\
\hline CVI1 & 3.30 & .819 & 288 \\
CVI2 & 2.88 & .703 & 288 \\
CVI3 & 3.06 & .691 & 288 \\
CVI4 & 4.16 & .652 & 288 \\
\hline
\end{tabular}

Inter-Item Correlation Matrix

\begin{tabular}{|l|c|c|c|c|}
\hline & CVI1 & CVI2 & CVI3 & CVI4 \\
\hline CVI1 & 1.000 & -.223 & -.036 & .206 \\
CVI2 & -.223 & 1.000 & .393 & -.157 \\
CVI3 & -.036 & .393 & 1.000 & -.066 \\
CVI4 & .206 & -.157 & -.066 & 1.000 \\
\hline
\end{tabular}

Summary Item Statistics

\begin{tabular}{|l|c|c|c|c|c|c|c|}
\hline & Mean & Minimum & Maximum & Range & $\begin{array}{c}\text { Maximum / } \\
\text { Minimum }\end{array}$ & Variance & No. of Items \\
\hline Item Means & 3.348 & 2.882 & 4.156 & 1.274 & 1.442 & .319 & 4 \\
Inter-Item Correlations & .020 & -.223 & .393 & .616 & -1.764 & .050 & 4 \\
\hline
\end{tabular}

Item-Total Statistics

\begin{tabular}{|c|c|c|c|c|c|}
\hline & $\begin{array}{c}\text { Scale Mean if } \\
\text { Item Deleted }\end{array}$ & $\begin{array}{c}\text { Scale Variance } \\
\text { if Item Deleted }\end{array}$ & $\begin{array}{c}\text { Corrected Item- } \\
\text { Total Correlation }\end{array}$ & $\begin{array}{c}\text { Squared Multiple } \\
\text { Correlation }\end{array}$ & $\begin{array}{c}\text { Cronbach's Alpha if } \\
\text { Item Deleted }\end{array}$ \\
\hline CVI1 & 10.09 & 1.577 & -.038 & .083 & .170 \\
CVI2 & 10.51 & 1.693 & -.010 & .207 & .107 \\
CVI3 & 10.34 & 1.409 & .172 & .158 & $-.193^{a}$ \\
CVI4 & 9.24 & 1.728 & .010 & .056 & .074 \\
\hline
\end{tabular}

a. The value is negative due to a negative average covariance among items. This violates reliability model assumptions. You may want to check item codings.

Scale Statistics

\begin{tabular}{|c|c|c|c|}
\hline Mean & Variance & Std. Deviation & No. of Items \\
\hline 13.39 & 2.170 & 1.473 & 4 \\
\hline
\end{tabular}




\section{TOTAL VII - Cultural - inter-item correlation}

\begin{tabular}{|c|c|c|c|}
\hline \multicolumn{4}{|c|}{ Case Processing Summary } \\
\hline & & $\mathrm{N}$ & $\%$ \\
\hline \multirow{3}{*}{ Cases } & Valid & 288 & 100 \\
\hline & Excludeda & 0 & 0 \\
\hline & Total & 288 & 100 \\
\hline
\end{tabular}

a. Listwise deletion based on all variables in the procedure.

Reliability Statistics

\begin{tabular}{|c|c|c|}
\hline Cronbach's Alpha & $\begin{array}{c}\text { Cronbach's Alpha Based } \\
\text { on Standardised Items }\end{array}$ & No. of Items \\
\hline .439 & .480 & 6 \\
\hline
\end{tabular}

\begin{tabular}{|c|c|c|c|}
\multicolumn{4}{|c|}{ Item Statistics } \\
\hline & Mean & Std. Deviation & $\mathrm{N}$ \\
\hline CVII1 & 3.91 & .714 & 288 \\
CVII2 & 3.92 & .671 & 288 \\
CVII3 & 2.43 & .908 & 288 \\
CVII4 & 2.29 & .830 & 288 \\
CVII5 & 4.08 & .578 & 288 \\
CVII6 & 3.80 & .684 & 288 \\
\hline
\end{tabular}

\begin{tabular}{|l|c|c|c|c|c|c|}
\hline \multicolumn{7}{|c|}{ Inter-Item Correlation Matrix } \\
\hline & CVII1 & CVII2 & CVII3 & CVII4 & CVII5 & CVII6 \\
\hline CVII1 & 1.000 & .660 & .019 & -.048 & .407 & .261 \\
CVII2 & .660 & 1.000 & -.030 & -.102 & .358 & .291 \\
CVII3 & .019 & -.030 & 1.000 & .584 & -.247 & -.136 \\
CVII4 & -.048 & -.102 & .584 & 1.000 & -.254 & -.265 \\
CVII5 & .407 & .358 & -.247 & -.254 & 1.000 & .500 \\
CVII6 & .261 & .291 & -.136 & -.265 & .500 & 1.000 \\
\hline
\end{tabular}

\begin{tabular}{|l|c|c|c|c|c|c|c|}
\hline & Mean & Minimum & Maximum & Range & $\begin{array}{c}\text { Maximum / } \\
\text { Minimum }\end{array}$ & Variance & No. of Items \\
& 3.405 & 2.292 & 4.083 & 1.792 & 1.782 & .666 & 6 \\
Item Means & .133 & -.265 & .660 & .925 & -2.497 & .099 & 6 \\
\hline
\end{tabular}

\begin{tabular}{|c|c|c|c|c|c|}
\hline & $\begin{array}{c}\text { Scale Mean if } \\
\text { Item Deleted }\end{array}$ & $\begin{array}{c}\text { Scale Variance } \\
\text { if Item Deleted }\end{array}$ & $\begin{array}{c}\text { Corrected Item- } \\
\text { Total } \\
\text { Correlation }\end{array}$ & $\begin{array}{c}\text { Squared Multiple } \\
\text { Correlation }\end{array}$ & $\begin{array}{c}\text { Cronbach's } \\
\text { Alpha if Item } \\
\text { Deleted }\end{array}$ \\
\hline CVII1 & 16.52 & 3.463 & .448 & .477 & .252 \\
CVII2 & 16.51 & 3.686 & .399 & .455 & .292 \\
CVII3 & 18.00 & 3.902 & .122 & .365 & .465 \\
CVII4 & 18.14 & 4.313 & .048 & .377 & .500 \\
CVII5 & 16.34 & 4.324 & .211 & .374 & .400 \\
CVII6 & 16.63 & 4.248 & .159 & .290 & .424 \\
\hline
\end{tabular}

Scale Statistics

\begin{tabular}{|c|c|c|c|}
\hline Mean & Variance & Std. Deviation & No. of Items \\
\hline 20.43 & 5.165 & 2.273 & 6 \\
\hline
\end{tabular}




\section{TOTAL VIII - Financial - inter-item correlation}

\begin{tabular}{|c|c|c|c|}
\hline \multicolumn{4}{|c|}{ Case Processing Summary } \\
\hline & & $\mathrm{N}$ & $\%$ \\
\hline \multirow{3}{*}{ Cases } & Valid & 288 & 100 \\
\hline & Excludeda & 0 & 0 \\
\hline & Total & 288 & 100 \\
\hline
\end{tabular}

a. Listwise deletion based on all variables in the procedure.

Reliability Statistics

\begin{tabular}{|c|c|c|}
\hline Cronbach's Alpha & $\begin{array}{c}\text { Cronbach's Alpha Based } \\
\text { on Standardised Items }\end{array}$ & No. of Items \\
\hline .626 & .610 & 7 \\
\hline
\end{tabular}

\begin{tabular}{|c|c|c|c|}
\hline \multicolumn{3}{|c|}{ Item Statistics } \\
\hline & Mean & Std. Deviation & $\mathrm{N}$ \\
\hline CVIII1 & 1.85 & 1.123 & 288 \\
CVIII2 & 2.67 & 1.129 & 288 \\
CVIII3 & 2.69 & 1.117 & 288 \\
CVIII4 & 2.92 & 1.056 & 288 \\
CVIII5 & 3.86 & .781 & 288 \\
CVIII6 & 3.22 & .893 & 288 \\
CVIII7 & 3.38 & .867 & 288 \\
\hline
\end{tabular}

Inter-Item Correlation Matrix

\begin{tabular}{|l|c|c|c|c|c|c|c|}
\hline & CVIII1 & CVIII2 & CVIII3 & CVIII4 & CVIII5 & CVIII6 & CVIII7 \\
\hline CVIII1 & 1.000 & .498 & .311 & .258 & .115 & .042 & -.026 \\
CVIII2 & .498 & 1.000 & .609 & .236 & .168 & .098 & .016 \\
CVIII3 & .311 & .609 & 1.000 & .310 & .200 & .050 & .028 \\
CVIII4 & .258 & .236 & .310 & 1.000 & .253 & -.031 & -.003 \\
CVIII5 & .115 & .168 & .200 & .253 & 1.000 & -.026 & .064 \\
CVIII6 & .042 & .098 & .050 & -.031 & -.026 & 1.000 & .661 \\
CVIII7 & -.026 & .016 & .028 & -.003 & .064 & .661 & 1.000 \\
\hline
\end{tabular}

Summary Item Statistics

\begin{tabular}{|l|c|c|c|c|c|c|c|}
\hline & Mean & Minimum & Maximum & Range & $\begin{array}{c}\text { Maximum / } \\
\text { Minimum }\end{array}$ & Variance & No. of Items \\
\hline Item Means & 2.940 & 1.854 & 3.858 & 2.003 & 2.081 & .404 & 7 \\
Inter-Item Correlations & .183 & -.031 & .661 & .692 & -21.650 & .041 & 7 \\
\hline
\end{tabular}

Item-Total Statistics

\begin{tabular}{|l|c|c|c|c|c|}
\hline & $\begin{array}{c}\text { Scale Mean if } \\
\text { Item Deleted }\end{array}$ & $\begin{array}{c}\text { Scale Variance if } \\
\text { Item Deleted }\end{array}$ & $\begin{array}{c}\text { Corrected Item-Total } \\
\text { Correlation }\end{array}$ & $\begin{array}{c}\text { Squared Multiple } \\
\text { Correlation }\end{array}$ & $\begin{array}{c}\text { Cronbach's Alpha } \\
\text { if Item Deleted }\end{array}$ \\
\hline CVIII1 & 18.73 & 11.083 & .386 & .271 & .573 \\
CVIII2 & 17.91 & 10.073 & .542 & .483 & .511 \\
CVIII3 & 17.90 & 10.435 & .492 & .405 & .532 \\
CVIII4 & 17.66 & 11.856 & .311 & .162 & .599 \\
CVIII5 & 16.73 & 13.308 & .231 & .095 & .619 \\
CVIII6 & 17.37 & 13.125 & .203 & .454 & .628 \\
CVIII7 & 17.21 & 13.343 & .180 & .450 & .633 \\
\hline
\end{tabular}

Scale Statistics

\begin{tabular}{|c|c|c|c|}
\hline Mean & Variance & Std. Deviation & No. of Items \\
\hline 20.58 & 15.233 & 3.903 & 7 \\
\hline
\end{tabular}




\section{Appendix 3: Factor analysis}

Part 1: TOTAL II - online learning style readiness

Correlation Matrix

\begin{tabular}{|c|c|c|c|c|c|c|c|c|c|c|}
\hline & & Cll1 & $\mathrm{Cll} 2$ & $\mathrm{ClI3}$ & $\mathrm{Cll} 4$ & Cll5 & Cll6 & CII7 & $\mathrm{Cll}$ & CII9 \\
\hline \multirow[t]{9}{*}{ Correlation } & Cll1 & 1.000 & .602 & .410 & .515 & .076 & .256 & .000 & -.057 & .131 \\
\hline & ClI2 & .602 & 1.000 & .454 & .547 & .080 & .291 & .012 & -.047 & .151 \\
\hline & CII3 & .410 & .454 & 1.000 & .475 & -.015 & .137 & -.047 & -.061 & .102 \\
\hline & Cll4 & .515 & .547 & .475 & 1.000 & .024 & .267 & .028 & -.084 & .162 \\
\hline & ClI5 & .076 & .080 & -.015 & .024 & 1.000 & .054 & .244 & .101 & -.041 \\
\hline & CII6 & .256 & .291 & .137 & .267 & .054 & 1.000 & .090 & .042 & .006 \\
\hline & ClI7 & .000 & .012 & -.047 & .028 & .244 & .090 & 1.000 & .522 & -.445 \\
\hline & Cll8 & -.057 & -.047 & -.061 & -.084 & .101 & .042 & .522 & 1.000 & -.496 \\
\hline & Cll9 & .131 & .151 & .102 & .162 & -.041 & .006 & -.445 & -.496 & 1.000 \\
\hline
\end{tabular}

KMO and Bartlett's Test

\begin{tabular}{|c|c|c|}
\hline \multicolumn{2}{|c|}{ Kaiser-Meyer-Olkin Measure of Sampling Adequacy. } & .757 \\
\hline Bartlett's Test of Sphericity & $\begin{array}{l}\text { Approx. Chi-Square } \\
\text { df } \\
\text { Siq. }\end{array}$ & $\begin{array}{r}608.893 \\
36.000 \\
.000\end{array}$ \\
\hline
\end{tabular}

\begin{tabular}{|c|c|c|}
\multicolumn{3}{|c|}{ Communalities } \\
\hline & Initial & Extraction \\
\hline CII1 & 1.000 & .632 \\
CII2 & 1.000 & .686 \\
CII3 & 1.000 & .470 \\
CII4 & 1.000 & .635 \\
CII5 & 1.000 & .122 \\
CII6 & 1.000 & .240 \\
CII7 & 1.000 & .687 \\
CII8 & 1.000 & .662 \\
CII9 & 1.000 & .592 \\
\hline
\end{tabular}

Extraction Method: Principal Component Analysis.

Total Variance Explained

\begin{tabular}{|c|c|c|c|c|c|c|}
\hline \multirow{2}{*}{ Component } & \multicolumn{3}{|c|}{ Initial Eigenvalues } & \multicolumn{2}{|c|}{ Extraction Sums of Squared Loadings } \\
\cline { 2 - 7 } & Total & $\begin{array}{c}\% \text { of } \\
\text { Variance }\end{array}$ & $\begin{array}{c}\text { Cumulative } \\
\%\end{array}$ & Total & $\begin{array}{c}\% \text { of } \\
\text { Variance }\end{array}$ & Cumulative \% \\
\hline 1 & 2.722 & 30.248 & 30.248 & 2.722 & 30.248 & 30.248 \\
2 & 2.004 & 22.268 & 52.517 & 2.004 & 22.268 & 52.517 \\
3 & .989 & 10.990 & 63.507 & & & \\
4 & .868 & 9.647 & 73.153 & & & \\
5 & .590 & 6.551 & 79.705 & & & \\
6 & .534 & 5.935 & 85.640 & & & \\
7 & .486 & 5.404 & 91.044 & & & \\
8 & .416 & 4.627 & 95.670 & & & \\
9 & .390 & 4.330 & 100.000 & & & \\
\hline
\end{tabular}

Extraction Method: Principal Component Analysis. 
Component Matrix

\begin{tabular}{|c|c|c|}
\hline \multirow{2}{*}{} & \multicolumn{2}{|c|}{ Component } \\
\cline { 2 - 3 } CII2 & 1 & 2 \\
Cll4 & .811 & \\
CII1 & .786 & \\
CII3 & .780 & \\
Cll6 & .682 & \\
CII7 & .419 & \\
CII8 & & .818 \\
CII9 & .348 & .778 \\
Cll5 & & -.686 \\
\hline
\end{tabular}

Extraction Method: Principal Component Analysis.

a. 2 components extracted.

Part 2 (factor rotation): TOTAL II - online learning style readiness

Rotated Component Matrix

\begin{tabular}{|c|c|c|}
\hline \multirow{2}{*}{} & \multicolumn{2}{|c|}{ Component } \\
\cline { 2 - 3 } CII2 & 1 & 2 \\
CII4 & .828 & \\
CII1 & .796 & \\
CII3 & .795 & \\
CII6 & .682 & \\
CII7 & .464 & \\
CII8 & & .827 \\
CII9 & & .811 \\
CII5 & & -.746 \\
\hline
\end{tabular}

Extraction Method: Principal Component Analysis.

Rotation Method: Varimax with Kaiser Normalisation.

a. Rotation converged in three iterations.

Total Variance Explained

\begin{tabular}{|c|c|c|c|}
\hline \multirow{2}{*}{ Component } & \multicolumn{3}{|c|}{ Rotation Sums of Squared Loadings } \\
\cline { 2 - 4 } & Total & \% of Variance & Cumulative \% \\
\hline 1 & 2.688 & 29.871 & 29.871 \\
2 & 2.038 & 22.645 & 52.517 \\
\hline
\end{tabular}

Extraction Method: Principal Component Analysis.

Component Transformation Matrix

\begin{tabular}{|c|c|c|}
\hline Component & 1 & 2 \\
\hline 1 & .976 & -.217 \\
2 & .217 & .976 \\
\hline
\end{tabular}

Extraction Method: Principal Component Analysis.

Rotation Method: Varimax with Kaiser Normalisation. 
Part 1: TOTAL VII - Cultural readiness

Correlation Matrix

\begin{tabular}{|c|c|c|c|c|c|c|c|}
\hline & & CVII1 & CVII2 & CVII3 & CVII4 & CVII5 & CVII6 \\
\hline \multirow[t]{6}{*}{ Correlation } & CVII1 & 1.000 & .660 & .019 & -.048 & .407 & .261 \\
\hline & CVII2 & .660 & 1.000 & -.030 & -.102 & .358 & .291 \\
\hline & CVII3 & .019 & -.030 & 1.000 & .584 & -.247 & -.136 \\
\hline & CVII4 & -.048 & -.102 & .584 & 1.000 & -.254 & -.265 \\
\hline & CVII5 & .407 & .358 & -.247 & -.254 & 1.000 & .500 \\
\hline & CVIII & .261 & .291 & -.136 & -.265 & .500 & 1.000 \\
\hline
\end{tabular}

KMO and Bartlett's Test

\begin{tabular}{|c|c|c|}
\hline \multicolumn{2}{|c|}{ Kaiser-Meyer-Olkin Measure of Sampling Adequacy. } & .632 \\
\hline Bartlett's Test of Sphericity & $\begin{array}{l}\text { Approx. Chi-Square } \\
\text { df } \\
\text { Sig. }\end{array}$ & $\begin{array}{r}464.688 \\
15.000 \\
.000\end{array}$ \\
\hline
\end{tabular}

\begin{tabular}{|l|c|c|}
\multicolumn{3}{|c|}{ Communalities } \\
\hline & Initial & Extraction \\
\hline CVII1 & 1.000 & .736 \\
CVII2 & 1.000 & .694 \\
CVII3 & 1.000 & .737 \\
CVII4 & 1.000 & .739 \\
CVII5 & 1.000 & .594 \\
CVII6 & 1.000 & .454 \\
\hline
\end{tabular}

Extraction Method: Principal Component Analysis.

Total Variance Explained

\begin{tabular}{|c|c|c|c|c|c|c|}
\hline \multirow{2}{*}{ Component } & \multicolumn{3}{|c|}{ Initial Eigenvalues } & \multicolumn{2}{c|}{ Extraction Sums of Squared Loadings } \\
\cline { 2 - 7 } & Total & $\begin{array}{c}\% \text { of } \\
\text { Variance }\end{array}$ & $\begin{array}{c}\text { Cumulative } \\
\%\end{array}$ & Total & \% of Variance & Cumulative \% \\
\hline 1 & 2.419 & 40.309 & 40.309 & 2.419 & 40.309 & 40.309 \\
2 & 1.536 & 25.608 & 65.917 & 1.536 & 25.608 & 65.917 \\
3 & .821 & 13.685 & 79.602 & & & \\
4 & .522 & 8.692 & 88.294 & & & \\
5 & .380 & 6.338 & 94.632 & & & \\
6 & .322 & 5.368 & 100.000 & & & \\
\hline
\end{tabular}

Extraction Method: Principal Component Analysis.

\begin{tabular}{|c|c|c|}
\hline \multicolumn{3}{|c|}{ Component Matrix ${ }^{a}$} \\
\hline & \multicolumn{2}{|c|}{ Component } \\
\hline & 1 & 2 \\
\hline CVII5 & .771 & \\
\hline CVII2 & .695 & .460 \\
\hline CVII1 & .680 & .523 \\
\hline CVII6 & .673 & \\
\hline CVII3 & -.413 & .753 \\
\hline CVII4 & -.506 & .695 \\
\hline
\end{tabular}

Extraction Method: Principal Component Analysis.

a. 2 components extracted. 
Part 2 (factor rotation): TOTAL VII - cultural readiness

Rotated Component Matrix ${ }^{\mathrm{a}}$
\begin{tabular}{|l|c|c|}
\hline \multirow{2}{*}{} & \multicolumn{2}{|c|}{ Component } \\
\cline { 2 - 3 } & 1 & \multicolumn{1}{|c|}{2} \\
\hline CVII1 & .849 & \\
CVII2 & .831 & \\
CVII5 & .674 & -.374 \\
CVII6 & .574 & -.352 \\
CVII3 & & .859 \\
CVII4 & & .854 \\
\hline
\end{tabular}

Extraction Method: Principal Component Analysis.

Rotation Method: Varimax with Kaiser Normalisation.

a. Rotation converged in three iterations.

Total Variance Explained

\begin{tabular}{|c|c|c|c|}
\hline \multirow{2}{*}{ Component } & \multicolumn{3}{|c|}{ Rotation Sums of Squared Loadings } \\
\cline { 2 - 4 } & Total & \% of Variance & Cumulative \% \\
\hline 1 & 2.206 & 36.759 & 36.759 \\
2 & 1.749 & 29.157 & 65.917 \\
\hline
\end{tabular}

Extraction Method: Principal Component Analysis.

\section{Component Transformation Matrix}

\begin{tabular}{|c|c|c|}
\hline Component & 1 & 2 \\
\hline 1 & .871 & -.491 \\
2 & .491 & .871 \\
\hline
\end{tabular}

Extraction Method: Principal Component Analysis.

Rotation Method: Varimax with Kaiser Normalisation.

\section{Part 1: TOTAL VIII - Financial readiness}

Correlation Matrix

\begin{tabular}{|rr|r|r|r|r|r|r|r|}
\hline & & CVIII1 & \multicolumn{1}{c|}{ CVIII2 } & CVIII3 & CVIII4 & CVIII5 & CVIII6 & CVIII7 \\
\hline Correlation & CVIII1 & 1.000 & .498 & .311 & .258 & .115 & .042 & -.026 \\
& CVIII2 & .498 & 1.000 & .609 & .236 & .168 & .098 & .016 \\
& CVIII3 & .311 & .609 & 1.000 & .310 & .200 & .050 & .028 \\
& CVIII4 & .258 & .236 & .310 & 1.000 & .253 & -.031 & -.003 \\
CVIII5 & .115 & .168 & .200 & .253 & 1.000 & -.026 & .064 \\
CVIII6 & .042 & .098 & .050 & -.031 & -.026 & 1.000 & .661 \\
CVIII7 & -.026 & .016 & .028 & -.003 & .064 & .661 & 1.000 \\
\hline
\end{tabular}

KMO and Bartlett's Test

\begin{tabular}{|c|c|c|}
\hline \multicolumn{2}{|c|}{ Kaiser-Meyer-Olkin Measure of Sampling Adequacy. } & .595 \\
\hline Bartlett's Test of Sphericity & $\begin{array}{l}\text { Approx. Chi-Square } \\
\text { df } \\
\text { Sig. }\end{array}$ & $\begin{array}{r}448.288 \\
21.000 \\
.000\end{array}$ \\
\hline
\end{tabular}




\begin{tabular}{|l|c|c|}
\multicolumn{3}{|c|}{ Communalities } \\
\hline & Initial & Extraction \\
\hline CVIII1 & 1.000 & .559 \\
CVIII2 & 1.000 & .773 \\
CVIII3 & 1.000 & .624 \\
CVIII4 & 1.000 & .526 \\
CVIII5 & 1.000 & .761 \\
CVIII6 & 1.000 & .837 \\
CVIII7 & 1.000 & .840 \\
\hline
\end{tabular}

Extraction Method: Principal Component Analysis.

Total Variance Explained

\begin{tabular}{|c|c|c|c|c|c|c|}
\hline \multirow{2}{*}{ Component } & \multicolumn{3}{|c|}{ Initial Eigenvalues } & \multicolumn{2}{c|}{ Extraction Sums of Squared Loadings } \\
\cline { 2 - 7 } & Total & $\begin{array}{c}\% \text { of } \\
\text { Variance }\end{array}$ & $\begin{array}{c}\text { Cumulative } \\
\%\end{array}$ & Total & $\begin{array}{c}\% \text { of } \\
\text { Variance }\end{array}$ & Cumulative \% \\
\hline 1 & 2.256 & 32.225 & 32.225 & 2.256 & 32.225 & 32.225 \\
2 & 1.657 & 23.670 & 55.895 & 1.657 & 23.670 & 55.895 \\
3 & 1.007 & 14.392 & 70.287 & 1.007 & 14.392 & 70.287 \\
4 & .737 & 10.522 & 80.809 & & & \\
5 & .683 & 9.755 & 90.564 & & & \\
6 & .346 & 4.943 & 95.507 & & & \\
7 & .315 & 4.493 & 100.000 & & & \\
\hline
\end{tabular}

Extraction Method: Principal Component Analysis.

Component Matrix ${ }^{\mathrm{a}}$

\begin{tabular}{|l|c|c|c|}
\hline \multirow{2}{*}{} & \multicolumn{3}{|c|}{ Component } \\
\cline { 2 - 4 } & 1 & 2 & 3 \\
\hline CVIIII & .819 & -.038 & -.317 \\
CVIII3 & .778 & -.059 & -.123 \\
CVIII1 & .674 & -.100 & -.308 \\
CVIII4 & .563 & -.145 & .433 \\
CVIII7 & .116 & .901 & .125 \\
CVIII6 & .157 & .899 & -.070 \\
CVIII5 & .412 & -.049 & .767 \\
\hline
\end{tabular}

Extraction Method: Principal Component Analysis. a. 3 components extracted.

\section{Part 2 (factor rotation): TOTAL VIII - Financial readiness}

Rotated Component Matrix ${ }^{\mathrm{a}}$

\begin{tabular}{|c|c|c|c|}
\hline \multirow{2}{*}{} & \multicolumn{3}{|c|}{ Component } \\
\cline { 2 - 4 } & 1 & 2 & 3 \\
\hline CVIII2 & .873 & .055 & .093 \\
CVIII3 & .749 & .037 & .249 \\
CVIII1 & .746 & & .041 \\
CVIII7 & -.040 & .913 & .074 \\
CVIII6 & .086 & .907 & -.080 \\
CVIII5 & & .039 & .871 \\
CVIII4 & .311 & -.051 & .653 \\
\hline
\end{tabular}

Extraction Method: Principal Component Analysis.

Rotation Method: Varimax with Kaiser Normalisation.

a. Rotation converged in four iterations. 
Total Variance Explained

\begin{tabular}{|c|c|c|c|}
\hline \multirow{2}{*}{ Component } & \multicolumn{3}{|c|}{ Rotation Sums of Squared Loadings } \\
\cline { 2 - 4 } & Total & \% of Variance & Cumulative \% \\
\hline 1 & 1.985 & 28.357 & 28.357 \\
2 & 1.666 & 23.795 & 52.152 \\
3 & 1.269 & 18.135 & 70.287 \\
\hline
\end{tabular}

Extraction Method: Principal Component Analysis.

\section{Component Transformation Matrix}

\begin{tabular}{|c|c|c|c|}
\hline Component & 1 & 2 & 3 \\
\hline 1 & .882 & .130 & .453 \\
2 & -.095 & .991 & -.099 \\
3 & -.461 & .045 & .886 \\
\hline
\end{tabular}

Extraction Method: Principal Component Analysis.

Rotation Method: Varimax with Kaiser Normalisation. 


\section{Appendix 4: Fourth reliability test - after factor analysis}

\section{TOTAL II - positive online learning style readiness}

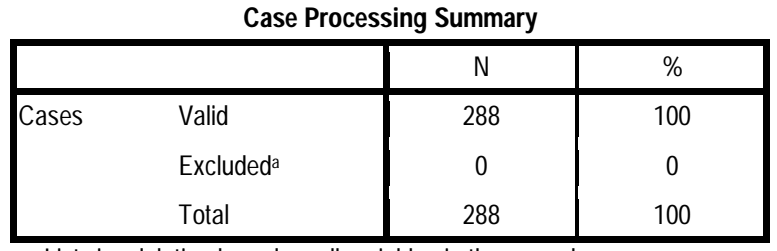

a. Listwise deletion based on all variables in the procedure.

Reliability Statistics

\begin{tabular}{|c|c|c|}
\hline Cronbach's Alpha & $\begin{array}{c}\text { Cronbach's Alpha Based } \\
\text { on Standardised Items }\end{array}$ & No. of Items \\
\hline .725 & .720 & 6 \\
\hline
\end{tabular}

Item Statistics

\begin{tabular}{|c|c|c|c|}
\hline & Mean & Std. Deviation & $\mathrm{N}$ \\
\hline CII1 & 4.09 & .732 & 288 \\
CII2 & 3.70 & .765 & 288 \\
CII3 & 3.72 & .746 & 288 \\
CII4 & 3.77 & .757 & 288 \\
CII6 & 3.30 & .857 & 288 \\
CII9 & 4.14 & .597 & 288 \\
\hline
\end{tabular}

Summary Item Statistics

\begin{tabular}{|l|c|c|c|c|c|c|c|}
\hline & Mean & Minimum & Maximum & Range & Maximum / Minimum & Variance & No. of Items \\
\hline Item Means & 3.789 & 3.302 & 4.139 & .837 & 1.253 & .092 & 6 \\
Inter-Item Correlations & .300 & .006 & .602 & .596 & 96.467 & .034 & 6 \\
\hline
\end{tabular}

Item-Total Statistics

\begin{tabular}{|c|c|c|c|c|c|}
\hline & $\begin{array}{c}\text { Scale Mean if Item } \\
\text { Deleted }\end{array}$ & $\begin{array}{c}\text { Scale Variance if Item } \\
\text { Deleted }\end{array}$ & $\begin{array}{c}\text { Corrected Item-Total } \\
\text { Correlation }\end{array}$ & $\begin{array}{c}\text { Squared Multiple } \\
\text { Correlation }\end{array}$ & $\begin{array}{c}\text { Cronbach's Alpha if } \\
\text { Item Deleted }\end{array}$ \\
\hline Cll1 & 18.64 & 5.778 & .605 & .424 & .643 \\
CII2 & 19.03 & 5.511 & .653 & .471 & .625 \\
CII3 & 19.01 & 6.115 & .479 & .291 & .681 \\
CII4 & 18.96 & 5.636 & .621 & .412 & .636 \\
Cll6 & 19.43 & 6.476 & .282 & .110 & .747 \\
CII9 & 18.59 & 7.587 & .151 & .035 & .758 \\
\hline
\end{tabular}

Scale Statistics

\begin{tabular}{|c|c|c|c|}
\hline Mean & Variance & Std. Deviation & No. of Items \\
\hline 22.73 & 8.440 & 2.905 & 6 \\
\hline
\end{tabular}




\section{TOTAL II - negative online learning style readiness}

\begin{tabular}{|c|c|c|c|}
\hline \multicolumn{4}{|c|}{ Case Processing Summary } \\
\hline & & $\mathrm{N}$ & $\%$ \\
\hline \multirow[t]{3}{*}{ Cases } & Valid & 288 & 100 \\
\hline & Excludeda & 0 & 0 \\
\hline & Total & 288 & 100 \\
\hline
\end{tabular}

a. Listwise deletion based on all variables in the procedure.

Reliability Statistics

\begin{tabular}{|c|c|c|}
\hline Cronbach's Alpha & $\begin{array}{c}\text { Cronbach's Alpha Based } \\
\text { on Standardised Items }\end{array}$ & No. of Items \\
\hline .534 & .550 & 3 \\
\hline
\end{tabular}

\begin{tabular}{|c|c|c|c|}
\hline \multicolumn{4}{|c|}{ Item Statistics } \\
\hline & Mean & Std. Deviation & $\mathrm{N}$ \\
\hline CII5 & 2.78 & .859 & 288 \\
CII7 & 2.12 & .756 & 288 \\
CII8 & 1.98 & .720 & 288 \\
\hline
\end{tabular}

\begin{tabular}{|c|c|c|c|}
\multicolumn{4}{|c|}{ Inter-Item Correlation Matrix } \\
\hline & Cll5lear & ClI7lear & Cll8lear \\
\hline CII5 & 1.000 & .244 & .101 \\
CII7 & .244 & 1.000 & .522 \\
CII8 & .101 & .522 & 1.000 \\
\hline
\end{tabular}

Summary Item Statistics

\begin{tabular}{|l|c|c|c|c|c|c|c|}
\hline & Mean & Minimum & Maximum & Range & Maximum / Minimum & Variance & No. of Items \\
\hline Item Means & 2.293 & 1.983 & 2.778 & .795 & 1.401 & .181 & 3 \\
Inter-Item Correlations & .289 & .101 & .522 & .421 & 5.183 & .037 & 3 \\
\hline
\end{tabular}

Item-Total Statistics

\begin{tabular}{|c|c|c|c|c|c|}
\hline & $\begin{array}{c}\text { Scale Mean if } \\
\text { Item Deleted }\end{array}$ & $\begin{array}{c}\text { Scale Variance if Item } \\
\text { Deleted }\end{array}$ & $\begin{array}{c}\text { Corrected Item- } \\
\text { Total Correlation }\end{array}$ & $\begin{array}{c}\text { Squared Multiple } \\
\text { Correlation }\end{array}$ & $\begin{array}{c}\text { Cronbach's Alpha if } \\
\text { Item Deleted }\end{array}$ \\
\hline Cll5 & 4.10 & 1.659 & .200 & .061 & .686 \\
CII7 & 4.76 & 1.381 & .499 & .310 & .180 \\
CII8 & 4.90 & 1.627 & .377 & .273 & .390 \\
\hline
\end{tabular}

Scale Statistics

\begin{tabular}{|c|c|c|c|}
\hline Mean & Variance & Std. Deviation & No. of Items \\
\hline 6.88 & 2.839 & 1.685 & 3 \\
\hline
\end{tabular}




\section{TOTAL VII - Positive cultural readiness}

\begin{tabular}{|c|c|c|c|}
\hline \multicolumn{4}{|c|}{ Case Processing Summary } \\
\hline & & $\mathrm{N}$ & $\%$ \\
\hline \multirow[t]{3}{*}{ Cases } & Valid & 288 & 100 \\
\hline & Excludeda & 0 & 0 \\
\hline & Total & 288 & 100 \\
\hline
\end{tabular}

a. Listwise deletion based on all variables in the procedure.

Reliability Statistics

\begin{tabular}{|c|c|c|}
\hline Cronbach's Alpha & $\begin{array}{c}\text { Cronbach's Alpha Based } \\
\text { on Standardised Items }\end{array}$ & No. of Items \\
\hline .735 & .738 & 4 \\
\hline
\end{tabular}

\begin{tabular}{|c|c|c|c|}
\hline \multicolumn{4}{|c|}{ Item Statistics } \\
\hline & Mean & Std. Deviation & $\mathrm{N}$ \\
\hline CVII1 & 3.91 & .714 & 288 \\
CVII2 & 3.92 & .671 & 288 \\
CVII5 & 4.08 & .578 & 288 \\
CVII6 & 3.80 & .684 & 288 \\
\hline
\end{tabular}

\begin{tabular}{|c|c|c|c|c|}
\hline \multicolumn{5}{|c|}{ Inter-Item Correlation Matrix } \\
\hline & CVII1 & CVII2 & CVII5 & CVII6 \\
\hline CVII1 & 1.000 & .660 & .407 & .261 \\
CVII2 & .660 & 1.000 & .358 & .291 \\
CVII5 & .407 & .358 & 1.000 & .500 \\
CVII6 & .261 & .291 & .500 & 1.000 \\
\hline
\end{tabular}

Summary Item Statistics

\begin{tabular}{|l|c|c|c|c|c|c|c|}
\hline & Mean & Minimum & Maximum & Range & Maximum / Minimum & Variance & No. of Items \\
\hline Item Means & 3.927 & 3.799 & 4.083 & .285 & 1.075 & .014 & 4 \\
Inter-Item Correlations & .413 & .261 & .660 & .400 & 2.534 & .020 & 4 \\
\hline
\end{tabular}

Item-Total Statistics

\begin{tabular}{|c|c|c|c|c|c|}
\hline & $\begin{array}{c}\text { Scale Mean if Item } \\
\text { Deleted }\end{array}$ & $\begin{array}{c}\text { Scale Variance if } \\
\text { Item Deleted }\end{array}$ & $\begin{array}{c}\text { Corrected Item- } \\
\text { Total Correlation }\end{array}$ & $\begin{array}{c}\text { Squared Multiple } \\
\text { Correlation }\end{array}$ & $\begin{array}{c}\text { Cronbach's Alpha if } \\
\text { Item Deleted }\end{array}$ \\
\hline CVII1 & 11.80 & 2.194 & .578 & .470 & .644 \\
CVII2 & 11.79 & 2.300 & .579 & .454 & .644 \\
CVII5 & 11.62 & 2.584 & .543 & .335 & .671 \\
CVII6 & 11.91 & 2.542 & .421 & .265 & .736 \\
\hline
\end{tabular}

Scale Statistics

\begin{tabular}{|c|c|c|c|}
\hline Mean & Variance & Std. Deviation & No. of Items \\
\hline 15.71 & 3.929 & 1.982 & 4 \\
\hline
\end{tabular}




\section{TOTAL VII - Negative cultural readiness}

\begin{tabular}{|c|c|c|c|}
\hline \multicolumn{4}{|c|}{ Case Processing Summary } \\
\hline & & $\mathrm{N}$ & $\%$ \\
\hline \multirow[t]{3}{*}{ Cases } & Valid & 288 & 100 \\
\hline & Excludeda & 0 & 0 \\
\hline & Total & 288 & 100 \\
\hline
\end{tabular}

a. Listwise deletion based on all variables in the procedure.

Reliability Statistics

\begin{tabular}{|c|c|c|}
\hline Cronbach's Alpha & $\begin{array}{c}\text { Cronbach's Alpha Based } \\
\text { on Standardised Items }\end{array}$ & No. of Items \\
\hline .735 & .737 & 2 \\
\hline
\end{tabular}

\begin{tabular}{|c|c|c|c|}
\hline \multicolumn{4}{|c|}{ Item Statistics } \\
\hline & Mean & Std. Deviation & $\mathrm{N}$ \\
\hline CVII3 & 2.43 & .908 & 288 \\
CVII4 & 2.29 & .830 & 288 \\
\hline
\end{tabular}

\begin{tabular}{|c|c|c|}
\multicolumn{3}{|c|}{ Inter-Item Correlation Matrix } \\
\hline & CVII3 & CVII4 \\
\hline CVII3 & 1.000 & .584 \\
CVII4 & .584 & 1.000 \\
\hline
\end{tabular}

Summary Item Statistics

\begin{tabular}{|l|c|c|c|c|c|c|c|}
\hline & Mean & Minimum & Maximum & Range & Maximum / Minimum & Variance & No. of Items \\
\hline Item Means & 2.359 & 2.292 & 2.427 & .135 & 1.059 & .009 & 2 \\
Inter-Item Correlations & .584 & .584 & .584 & .000 & 1.000 & .000 & 2 \\
\hline
\end{tabular}

Item-Total Statistics

\begin{tabular}{|c|c|c|c|c|c|}
\hline & $\begin{array}{c}\text { Scale Mean if Item } \\
\text { Deleted }\end{array}$ & $\begin{array}{c}\text { Scale Variance if } \\
\text { Item Deleted }\end{array}$ & $\begin{array}{c}\text { Corrected Item-Total } \\
\text { Correlation }\end{array}$ & $\begin{array}{c}\text { Squared Multiple } \\
\text { Correlation }\end{array}$ & $\begin{array}{c}\text { Cronbach's Alpha if } \\
\text { Item Deleted }\end{array}$ \\
\hline CVII3 & 2.29 & .688 & .584 & .341 & .3 \\
CVII4 & 2.43 & .824 & .584 & .341 &. $\mathrm{a}$ \\
\hline
\end{tabular}

a. The value is negative due to a negative average covariance among items. This violates reliability model assumptions. You may want to check item codings.

Scale Statistics

\begin{tabular}{|c|c|c|c|}
\hline Mean & Variance & Std. Deviation & No. of Items \\
\hline 4.72 & 2.391 & 1.546 & 2 \\
\hline
\end{tabular}




\section{TOTAL VIlla - Financial affordability}

\begin{tabular}{|c|c|c|c|}
\hline \multicolumn{4}{|c|}{ Case Processing Summary } \\
\hline \multirow[t]{3}{*}{ Cases } & Valid & 288 & 100 \\
\hline & Excludeda & 0 & 0 \\
\hline & Total & 288 & 100 \\
\hline
\end{tabular}

a. Listwise deletion based on all variables in the procedure.

Reliability Statistics

\begin{tabular}{|c|c|c|}
\hline Cronbach's Alpha & $\begin{array}{c}\text { Cronbach's Alpha Based } \\
\text { on Standardised Items }\end{array}$ & No. of Items \\
\hline .729 & .729 & 3 \\
\hline
\end{tabular}

Item Statistics

\begin{tabular}{|c|c|c|c|}
\hline & Mean & Std. Deviation & $\mathrm{N}$ \\
\hline CVIII1 & 1.85 & 1.123 & 288 \\
CVIII2 & 2.67 & 1.129 & 288 \\
CVIII3 & 2.69 & 1.117 & 288 \\
\hline
\end{tabular}

Inter-Item Correlation Matrix

\begin{tabular}{|c|c|c|c|}
\hline & CVIII1 & CVIII2 & CVIII3 \\
\hline CVIII1 & 1.000 & .498 & .311 \\
CVIII2 & .498 & 1.000 & .609 \\
CVIII3 & .311 & .609 & 1.000 \\
\hline
\end{tabular}

Summary Item Statistics

\begin{tabular}{|l|c|c|c|c|c|c|c|}
\hline & Mean & Minimum & Maximum & Range & Maximum / Minimum & Variance & No. of Items \\
\hline Item Means & 2.404 & 1.854 & 2.688 & .833 & 1.449 & .227 & 3 \\
Inter-Item Correlations & .473 & .311 & .609 & .298 & 1.959 & .018 & 3 \\
\hline
\end{tabular}

Item-Total Statistics

\begin{tabular}{|c|c|c|c|c|c|}
\hline & $\begin{array}{c}\text { Scale Mean if Item } \\
\text { Deleted }\end{array}$ & $\begin{array}{c}\text { Scale Variance if Item } \\
\text { Deleted }\end{array}$ & $\begin{array}{c}\text { Corrected Item- } \\
\text { Total Correlation }\end{array}$ & $\begin{array}{c}\text { Squared Multiple } \\
\text { Correlation }\end{array}$ & $\begin{array}{c}\text { Cronbach's Alpha if } \\
\text { Item Deleted }\end{array}$ \\
\hline CVIII1 & 5.36 & 4.056 & .451 & .248 & .757 \\
CVIII2 & 4.54 & 3.287 & .683 & .476 & .474 \\
CVIII3 & 4.52 & 3.797 & .532 & .371 & .665 \\
\hline
\end{tabular}

Scale Statistics

\begin{tabular}{|c|c|c|c|}
\hline Mean & Variance & Std. Deviation & No. of Items \\
\hline 7.21 & 7.359 & 2.713 & 3 \\
\hline
\end{tabular}




\section{TOTAL VIIllb - Financial availability}

Case Processing Summary

\begin{tabular}{|ll|c|c|}
\hline & & $\mathrm{N}$ & $\%$ \\
\hline Cases & Valid & 288 & 100 \\
& Excludeda & 0 & 0 \\
& Total & 288 & 100 \\
\hline
\end{tabular}

a. Listwise deletion based on all variables in the procedure.

Reliability Statistics

\begin{tabular}{|c|c|c|}
\hline Cronbach's Alpha & $\begin{array}{c}\text { Cronbach's Alpha Based } \\
\text { on Standardised Items }\end{array}$ & No. of Items \\
\hline .389 & .404 & 2 \\
\hline
\end{tabular}

\begin{tabular}{|c|c|c|c|}
\hline \multicolumn{4}{|c|}{ Item Statistics } \\
\hline & Mean & Std. Deviation & $\mathrm{N}$ \\
\hline CVIII4 & 2.92 & 1.056 & 288 \\
CVIII5 & 3.86 & .781 & 288 \\
\hline
\end{tabular}

Inter-Item Correlation Matrix

\begin{tabular}{|r|r|r|}
\hline & \multicolumn{1}{|c|}{ CVIII4 } & \multicolumn{1}{|c|}{ CVIII5 } \\
\hline CVIII4 & 1.000 & .253 \\
CVIII5 & .253 & 1.000 \\
\hline
\end{tabular}

\begin{tabular}{|l|c|c|c|c|c|c|c|}
\hline & & & & & & Summary Item Statistics & $\begin{array}{c}\text { No. of } \\
\text { Items }\end{array}$ \\
\hline & Mean & Minimum & Maximum & Range & Maximum / Minimum & Variance \\
\hline Item Means & 3.391 & 2.924 & 3.858 & .934 & 1.319 & .436 & 2 \\
Inter-Item Correlations & .253 & .253 & .253 & .000 & 1.000 & .000 & 2 \\
\hline
\end{tabular}

Item-Total Statistics

\begin{tabular}{|c|c|c|c|c|c|}
\hline & $\begin{array}{c}\text { Scale Mean if Item } \\
\text { Deleted }\end{array}$ & $\begin{array}{c}\text { Scale Variance if } \\
\text { Item Deleted }\end{array}$ & $\begin{array}{c}\text { Corrected Item- } \\
\text { Total Correlation }\end{array}$ & $\begin{array}{c}\text { Squared Multiple } \\
\text { Correlation }\end{array}$ & $\begin{array}{c}\text { Cronbach's Alpha if Item } \\
\text { Deleted }\end{array}$ \\
\hline CVIII4 & 3.86 & .610 & .253 & .064 &..$^{\mathrm{a}}$ \\
CVIII5 & 2.92 & 1.116 & .253 & .064 &..$^{\mathrm{a}}$ \\
\hline
\end{tabular}

a. The value is negative due to a negative average covariance among items. This violates reliability model assumptions. You may want to check item codings.

Scale Statistics

\begin{tabular}{|c|c|c|c|}
\hline Mean & Variance & Std. Deviation & No. of Items \\
\hline 6.78 & 2.144 & 1.464 & 2 \\
\hline
\end{tabular}




\section{TOTAL VIIIC - Financial possibility}

Case Processing Summary

\begin{tabular}{|c|c|c|c|}
\hline & & $\mathrm{N}$ & $\%$ \\
\hline \multirow[t]{3}{*}{ Cases } & Valid & 288 & 100 \\
\hline & Excludeda & 0 & 0 \\
\hline & Total & 288 & 100 \\
\hline
\end{tabular}

a. Listwise deletion based on all variables in the procedure.

Reliability Statistics

\begin{tabular}{|c|c|c|}
\hline Cronbach's Alpha & $\begin{array}{c}\text { Cronbach's Alpha Based } \\
\text { on Standardised Items }\end{array}$ & No. of Items \\
\hline .796 & .796 & 2 \\
\hline
\end{tabular}

Item Statistics

\begin{tabular}{|c|c|c|c|}
\hline & Mean & Std. Deviation & $\mathrm{N}$ \\
\hline CVIII6 & 3.22 & .893 & 288 \\
CVIII7 & 3.38 & .867 & 288 \\
\hline
\end{tabular}

Inter-Item Correlation Matrix

\begin{tabular}{|c|c|c|}
\hline & CVIII6 & CVIII7 \\
\hline CVIII6 & 1.000 & .661 \\
CVIII7 & .661 & 1.000 \\
\hline
\end{tabular}

Summary Item Statistics

\begin{tabular}{|l|c|c|c|c|c|c|c|}
\hline & Mean & Minimum & Maximum & Range & Maximum / Minimum & Variance & No. of Items \\
\hline Item Means & 3.295 & 3.215 & 3.375 & .160 & 1.050 & .013 & 2 \\
Inter-Item Correlations & .661 & .661 & .661 & .000 & 1.000 & .000 & 2 \\
\hline
\end{tabular}

Item-Total Statistics

\begin{tabular}{|c|c|c|c|c|c|}
\hline & $\begin{array}{c}\text { Scale Mean if Item } \\
\text { Deleted }\end{array}$ & $\begin{array}{c}\text { Scale Variance if Item } \\
\text { Deleted }\end{array}$ & $\begin{array}{c}\text { Corrected Item-Total } \\
\text { Correlation }\end{array}$ & $\begin{array}{c}\text { Squared Multiple } \\
\text { Correlation }\end{array}$ & $\begin{array}{c}\text { Cronbach's Alpha if } \\
\text { Item Deleted }\end{array}$ \\
\hline CVIII6 & 3.38 & .751 & .661 & .437 &..$^{\mathrm{a}}$ \\
CVIII7 & 3.22 & .797 & .661 & .437 &.$^{\mathrm{a}}$ \\
\hline
\end{tabular}

a. The value is negative due to a negative average covariance among items. This violates reliability model assumptions. You may want to check item codings.

Scale Statistics

\begin{tabular}{|c|c|c|c|}
\hline Mean & Variance & Std. Deviation & No. of Items \\
\hline 6.59 & 2.570 & 1.603 & 2 \\
\hline
\end{tabular}


Appendix 5: Summary of mean scores

Mean scores for each scale

\begin{tabular}{|l|r|r|c|c|c|c|}
\hline $\begin{array}{c}\text { Dependent } \\
\text { variable }\end{array}$ & Mean & Std. Dev. & Min & Max & Range & $\begin{array}{c}\% \text { of } \\
\text { readiness }\end{array}$ \\
\hline TOTAL I & 19.6875 & 5.57804 & 6.00 & 30.00 & 24.00 & 65.63 \\
\hline TOTAL IIa & 19.0104 & 2.47283 & 9.00 & 25.00 & 16.00 & 76.04 \\
\hline TOTAL IIb & 6.8785 & 1.68489 & 3.00 & 13.00 & 10.00 & 45.86 \\
\hline TOTAL III & 31.3715 & 5.71728 & 14.00 & 40.00 & 26.00 & 78.43 \\
\hline TOTAL IV & 89.5347 & 9.24495 & 64.00 & 114.00 & 50.00 & 77.86 \\
\hline TOTAL V & 29.3611 & 5.85494 & 16.00 & 51.00 & 35.00 & 53.38 \\
\hline TOTAL VI & 13.3924 & 1.47294 & 9.00 & 20.00 & 11.00 & 66.96 \\
\hline TOTAL VIII & 15.7083 & 1.98206 & 10.00 & 20.00 & 10.00 & 78.54 \\
\hline TOTAL VIIb & 4.7188 & 1.54629 & 2.00 & 8.00 & 6.00 & 47.19 \\
\hline TOTAL VIIIa & 7.2118 & 2.71278 & 3.00 & 15.00 & 12.00 & 48.08 \\
\hline TOTAL VIIIb & 6.7813 & 1.46411 & 2.00 & 10.00 & 8.00 & 67.81 \\
\hline TOTAL VIIIC & 6.5903 & 1.60319 & 2.00 & 10.00 & 8.00 & 65.90 \\
\hline
\end{tabular}

Mean scores - gender

\begin{tabular}{|c|c|c|c|c|}
\hline \multirow{3}{*}{$\begin{array}{c}\text { Dependent } \\
\text { variable }\end{array}$} & \multicolumn{4}{|c|}{ GENDER } \\
\hline & \multicolumn{2}{|c|}{$\begin{array}{l}\text { Females } \\
(\mathrm{N}=84)\end{array}$} & \multicolumn{2}{|c|}{$\begin{array}{c}\text { Males } \\
(\mathrm{N}=204)\end{array}$} \\
\hline & Mean & Std. Dev. & Mean & Std. Dev. \\
\hline TOTAL I & 18.9167 & 5.04488 & 20.0049 & 5.76510 \\
\hline TOTAL Ila & 19.0238 & 2.44445 & 19.0049 & 2.49037 \\
\hline TOTAL Ilb & 6.7738 & 1.63804 & 6.9216 & 1.70589 \\
\hline TOTAL III & 31.0952 & 5.34952 & 31.4853 & 5.87093 \\
\hline TOTAL IV & 88.4048 & 8.56319 & 90.0000 & 9.49254 \\
\hline TOTAL V & 28.3095 & 5.34131 & 29.7941 & 6.01286 \\
\hline TOTAL VI & 13.2024 & 1.25899 & 13.4706 & 1.54859 \\
\hline TOTAL VIla & 15.7262 & 2.13040 & 15.7010 & 1.92310 \\
\hline TOTAL VIIb & 4.8690 & 1.59653 & 4.6569 & 1.52480 \\
\hline TOTAL VIIIa & 7.6190 & 2.66573 & 7.0441 & 2.72066 \\
\hline TOTAL VIIIlb & 7.0476 & 1.34348 & 6.6716 & 1.50040 \\
\hline TOTAL VIIIC & 6.9643 & 1.50858 & 6.4363 & 1.61912 \\
\hline
\end{tabular}

Mean scores - age groups

\begin{tabular}{|c|c|c|c|c|c|c|c|c|}
\hline \multirow{3}{*}{$\begin{array}{l}\text { Dependent } \\
\text { variable }\end{array}$} & \multicolumn{8}{|c|}{ AGE } \\
\hline & \multicolumn{2}{|c|}{$\begin{array}{c}18-25 \\
(N=22)\end{array}$} & \multicolumn{2}{|c|}{$\begin{array}{c}26-35 \\
(N=140)\end{array}$} & \multicolumn{2}{|c|}{$\begin{array}{c}36-45 \\
(N=68)\end{array}$} & \multicolumn{2}{|c|}{$\begin{array}{c}46-55 \\
(N=58)\end{array}$} \\
\hline & Mean & Std. Dev. & Mean & Std. Dev. & Mean & Std. Dev. & Mean & Std. Dev. \\
\hline TOTAL I & 20.1818 & 4.85638 & 21.9000 & 4.61200 & 18.7353 & 5.64264 & 15.2759 & 5.06724 \\
\hline TOTAL Ila & 19.4091 & 1.68068 & 19.6071 & 2.19449 & 18.7941 & 2.81021 & 17.6724 & 2.43071 \\
\hline TOTAL IIb & 6.9091 & 1.74326 & 6.8500 & 1.75444 & 6.9412 & 1.53450 & 6.8621 & 1.70086 \\
\hline TOTAL III & 31.6818 & 4.13333 & 32.5071 & 4.90118 & 31.6176 & 6.14509 & 28.2241 & 6.47815 \\
\hline TOTAL IV & 91.3182 & 7.02639 & 91.3857 & 8.61869 & 89.9559 & 9.56741 & 83.8966 & 9.01206 \\
\hline TOTAL V & 28.3636 & 5.80267 & 29.7786 & 5.89728 & 30.0147 & 6.25692 & 27.9655 & 5.10578 \\
\hline TOTAL VI & 13.3182 & 1.28680 & 13.4643 & 1.64649 & 13.3088 & 1.28432 & 13.3448 & 1.31858 \\
\hline TOTAL VIla & 15.2727 & 2.11979 & 15.8929 & 2.00250 & 16.0441 & 1.83200 & 15.0345 & 1.90995 \\
\hline TOTAL VIIb & 4.5909 & 1.46902 & 4.8000 & 1.62799 & 4.5735 & 1.51919 & 4.7414 & 1.42115 \\
\hline TOTAL VIIIIa & 7.0909 & 2.44772 & 7.4643 & 2.75475 & 7.3382 & 2.95039 & 6.5000 & 2.32643 \\
\hline TOTAL VIIIb & 6.5909 & 1.46902 & 6.7929 & 1.42181 & 6.8088 & 1.57648 & 6.7931 & 1.46010 \\
\hline TOTAL VIIIIC & 6.7727 & 1.30683 & 6.4500 & 1.70199 & 6.7206 & 1.69147 & 6.7069 & 1.33783 \\
\hline
\end{tabular}

Mean scores - education groups

\begin{tabular}{|c|c|c|c|c|c|c|}
\hline \multirow{3}{*}{$\begin{array}{l}\text { Dependent } \\
\text { variable }\end{array}$} & \multicolumn{6}{|c|}{ EDUCATION } \\
\hline & \multicolumn{2}{|c|}{$\begin{array}{l}\text { High School or less } \\
\qquad(N=65)\end{array}$} & \multicolumn{2}{|c|}{$\begin{array}{c}\text { Bachelors degrees \& } \\
\text { Diplomas } \\
(\mathrm{N}=154)\end{array}$} & \multicolumn{2}{|c|}{$\begin{array}{l}\text { Postgraduates } \\
\qquad(\mathrm{N}=69)\end{array}$} \\
\hline & Mean & Std. Dev. & Mean & Std. Dev. & Mean & Std. Dev. \\
\hline TOTAL I & 17.0308 & 5.79323 & 20.0455 & 5.20977 & 21.3913 & 5.35564 \\
\hline TOTAL Ila & 17.9692 & 2.70399 & 19.1558 & 2.16970 & 19.6667 & 2.61031 \\
\hline TOTAL Ilb & 6.9692 & 1.75863 & 6.9091 & 1.61028 & 6.7246 & 1.78955 \\
\hline TOTAL III & 28.8462 & 6.27801 & 31.7143 & 5.45177 & 32.9855 & 4.99851 \\
\hline TOTAL IV & 85.1231 & 8.36568 & 90.1104 & 9.08389 & 92.4058 & 9.02663 \\
\hline TOTAL V & 27.2923 & 6.18648 & 29.5844 & 5.70139 & 30.8116 & 5.40227 \\
\hline TOTAL VI & 13.0923 & 1.41115 & 13.4156 & 1.38480 & 13.6232 & 1.68122 \\
\hline TOTAL VIla & 14.6154 & 1.84300 & 15.8766 & 1.91768 & 16.3623 & 1.86272 \\
\hline TOTAL VIIb & 4.5077 & 1.65003 & 4.7857 & 1.59642 & 4.7681 & 1.31892 \\
\hline TOTAL VIIIa & 6.1231 & 2.33524 & 7.4416 & 2.88106 & 7.7246 & 2.39414 \\
\hline TOTAL VIIIb & 6.0615 & 1.36790 & 7.0065 & 1.34065 & 6.9565 & 1.61277 \\
\hline TOTAL VIIIC & 6.4154 & 1.46727 & 6.5584 & 1.68417 & 6.8261 & 1.53351 \\
\hline
\end{tabular}


Mean scores - respondents having Internet access at work

\begin{tabular}{|l|c|c|c|c|}
\hline \multirow{3}{*}{$\begin{array}{c}\text { Dependent } \\
\text { variable }\end{array}$} & \multicolumn{2}{|c|}{$\begin{array}{c}\text { YES } \\
(\mathbf{N}=\mathbf{2 5 0})\end{array}$} & \multicolumn{2}{c|}{$\begin{array}{c}\text { NO } \\
\mathbf{N}=\mathbf{3 8})\end{array}$} \\
\cline { 2 - 5 } & Mean & Std. Dev. & Mean & Std. Dev. \\
\cline { 2 - 5 } & 20.4760 & 5.14698 & 14.5000 & 5.58836 \\
\hline TOTAL I & 19.2160 & 2.43167 & 17.6579 & 2.33974 \\
\hline TOTAL Ila & 6.9240 & 1.67854 & 6.5789 & 1.71845 \\
\hline TOTAL IIb & 32.2640 & 4.96436 & 25.5000 & 6.84875 \\
\hline TOTAL III & 90.4920 & 8.90343 & 83.2368 & 9.08647 \\
\hline TOTAL IV & 29.9360 & 5.80990 & 25.5789 & 4.67097 \\
\hline TOTAL V & 13.4400 & 1.52831 & 13.0789 & .99679 \\
\hline TOTAL VI & 15.9560 & 1.89325 & 14.0789 & 1.79160 \\
\hline TOTAL VIIla & 4.7360 & 1.58631 & 4.6053 & 1.26362 \\
\hline TOTAL VIIlb & 7.4480 & 2.66151 & 5.6579 & 2.56037 \\
\hline TOTAL VIIIIa & 6.8280 & 1.45288 & 6.4737 & 1.51990 \\
\hline TOTAL VIIIb & 6.6360 & 1.64753 & 6.2895 & 1.25004 \\
\hline TOTAL VIIIC & & & & \\
\hline
\end{tabular}

Mean scores - respondents having Internet access at home

\begin{tabular}{|l|c|c|c|c|}
\hline \multirow{3}{*}{$\begin{array}{c}\text { Dependent } \\
\text { variable }\end{array}$} & \multicolumn{2}{|c|}{$\begin{array}{c}\text { YES } \\
(\mathbf{N}=\mathbf{5 6})\end{array}$} & \multicolumn{2}{c|}{$\begin{array}{c}\text { NO } \\
(\mathbf{N}=\mathbf{2 3 2})\end{array}$} \\
\cline { 2 - 5 } & Mean & Std. Dev. & Mean & Std. Dev. \\
\cline { 2 - 5 } & 23.0000 & 4.98361 & 18.8879 & 5.42604 \\
\hline TOTAL I & 20.4107 & 2.34929 & 18.6724 & 2.38602 \\
\hline TOTAL IIa & 6.7321 & 1.79384 & 6.9138 & 1.65965 \\
\hline TOTAL IIb & 33.1607 & 4.89072 & 30.9397 & 5.82692 \\
\hline TOTAL III & 93.5179 & 9.58785 & 88.5733 & 8.91780 \\
\hline TOTAL IV & 30.8393 & 5.62067 & 29.0043 & 5.86611 \\
\hline TOTAL V & 13.2321 & 1.50141 & 13.4310 & 1.46665 \\
\hline TOTAL VI & 16.4821 & 1.99992 & 15.5216 & 1.93609 \\
\hline TOTAL VIII & 5.0893 & 1.59860 & 4.6293 & 1.52344 \\
\hline TOTAL VIIb & 8.3750 & 2.65989 & 6.9310 & 2.65547 \\
\hline TOTAL VIIIa & 7.0000 & 1.53741 & 6.7284 & 1.44434 \\
\hline TOTAL VIIIb & 6.6429 & 1.76252 & 6.5776 & 1.56615 \\
\hline TOTAL VIIIC & & & & \\
\hline
\end{tabular}

Mean scores - job title

\begin{tabular}{|c|c|c|c|c|c|c|c|c|}
\hline \multirow{3}{*}{$\begin{array}{l}\text { Dependent } \\
\text { variable }\end{array}$} & \multicolumn{8}{|c|}{ JOB TITLE } \\
\hline & \multicolumn{2}{|c|}{$\begin{array}{c}\text { Managers } \\
(\mathrm{N}=42)\end{array}$} & \multicolumn{2}{|c|}{$\begin{array}{l}\text { IT professionals and } \\
\text { research assistants } \\
(\mathrm{N}=55)\end{array}$} & \multicolumn{2}{|c|}{$\begin{array}{l}\text { Researchers } \\
\quad(\mathrm{N}=91)\end{array}$} & \multicolumn{2}{|c|}{$\begin{array}{l}\text { General employees } \\
\qquad(N=100)\end{array}$} \\
\hline & Mean & Std. Dev. & Mean & Std. Dev. & Mean & Std. Dev. & Mean & Std. Dev. \\
\hline TOTAL I & 18.3571 & 5.30909 & 19.2727 & 6.21961 & 21.8022 & 4.79402 & 18.5500 & 5.50184 \\
\hline TOTAL \|la & 18.8571 & 1.69031 & 18.8727 & 2.53899 & 19.7802 & 2.42121 & 18.4500 & 2.61068 \\
\hline TOTAL Ilb & 6.6905 & 1.65997 & 7.0909 & 1.73496 & 6.8791 & 1.61820 & 6.8400 & 1.73915 \\
\hline TOTAL III & 31.4524 & 4.52205 & 30.6909 & 6.60492 & 32.5275 & 5.12150 & 30.6600 & 6.05400 \\
\hline TOTAL IV & 88.2381 & 8.54489 & 89.0182 & 9.05434 & 93.1978 & 8.84712 & 87.0300 & 9.06258 \\
\hline TOTAL V & 29.5714 & 4.83959 & 29.0909 & 6.49579 & 30.2857 & 5.20744 & 28.5800 & 6.36782 \\
\hline TOTAL VI & 13.5714 & 1.36405 & 13.2727 & 1.31169 & 13.5824 & 1.70663 & 13.2100 & 1.35807 \\
\hline TOTAL VIIa & 15.7143 & 1.58169 & 15.3091 & 1.98021 & 16.3187 & 1.89666 & 15.3700 & 2.09692 \\
\hline TOTAL VIllb & 4.9048 & 1.33999 & 4.7818 & 1.65206 & 4.7363 & 1.62505 & 4.5900 & 1.50484 \\
\hline TOTAL VIIIa & 7.0714 & 2.70855 & 6.9818 & 2.66313 & 7.8352 & 2.37704 & 6.8300 & 2.95780 \\
\hline TOTAL VIIIb & 7.1429 & 1.44106 & 6.3818 & 1.54549 & 6.9780 & 1.49055 & 6.6700 & 1.35628 \\
\hline TOTAL VIIIC & 6.6905 & 1.25888 & 6.4364 & 1.58422 & 6.6593 & 1.69457 & 6.5700 & 1.67124 \\
\hline
\end{tabular}


Appendix 6: Assumption checking for multiple regression

Outliers - cases with extreme values

\begin{tabular}{|c|c|c|c|c|}
\hline & & & Case Number & Value \\
\hline \multirow{10}{*}{$\begin{array}{l}\text { Mahalanobis } \\
\text { Distance }\end{array}$} & \multirow[t]{5}{*}{ Highest } & 1 & 223 & 20.53352 \\
\hline & & 2 & 149 & 19.11410 \\
\hline & & 3 & 158 & 17.99951 \\
\hline & & 4 & 221 & 17.88178 \\
\hline & & 5 & 214 & 17.28489 \\
\hline & \multirow[t]{5}{*}{ Lowest } & 1 & 282 & 3.84032 \\
\hline & & 2 & 94 & 3.84032 \\
\hline & & 3 & 93 & 3.84032 \\
\hline & & 4 & 49 & 3.84032 \\
\hline & & 5 & 156 & $4.14011^{\mathrm{a}}$ \\
\hline
\end{tabular}

Outliers - cases with extreme values

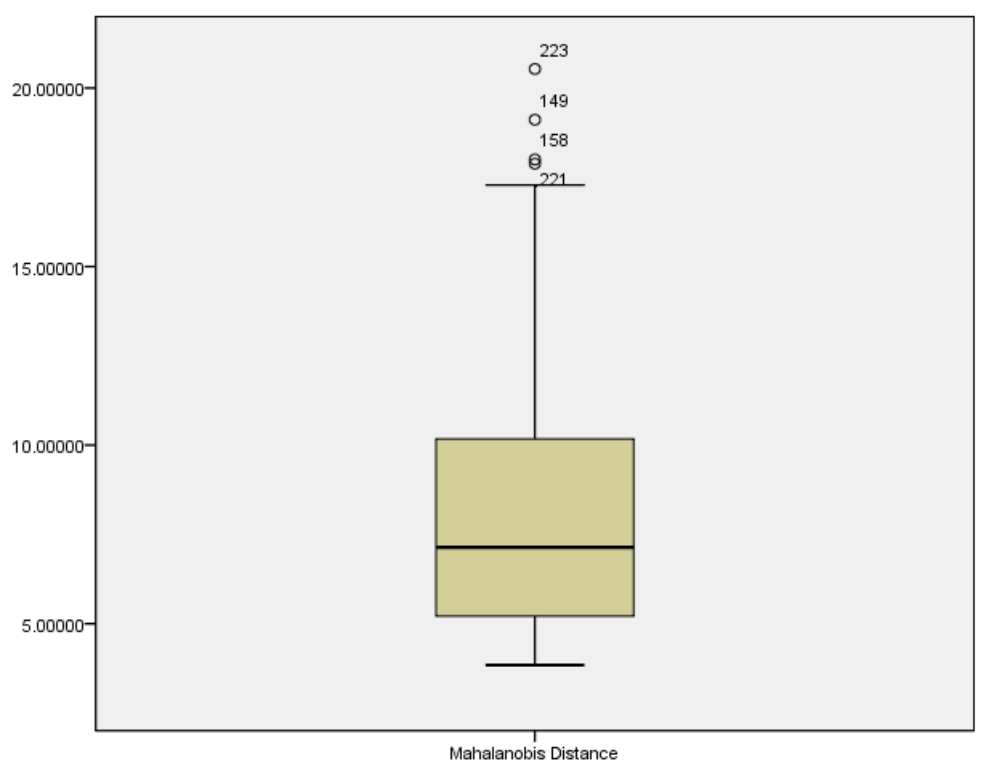

Assumption of no multicollinearity

\begin{tabular}{|l|c|c|}
\hline \multirow{2}{*}{\multicolumn{1}{|c|}{ IVs }} & \multicolumn{2}{c|}{ Collinearity Statistics } \\
\cline { 2 - 3 } & Tolerance & VIF \\
\hline Age & .735 & 1.361 \\
\hline Education & .532 & 1.879 \\
\hline B1atwork & .887 & 1.127 \\
\hline B2athome & .893 & 1.119 \\
\hline Gender & .902 & 1.108 \\
\hline Researchers & .549 & 1.822 \\
\hline Managers & .581 & 1.721 \\
\hline IT personnel and research assistants & .691 & 1.446 \\
\hline
\end{tabular}


Assumption checking for multiple regression

\begin{tabular}{|c|c|c|c|c|c|c|}
\hline \multirow{3}{*}{ DVs } & \multicolumn{2}{|c|}{ Normality of Errors } & \multicolumn{2}{|c|}{$\begin{array}{l}\text { Homoscedasticity and } \\
\text { Independence of Errors }\end{array}$} & \multirow{2}{*}{\multicolumn{2}{|c|}{$\begin{array}{c}\text { Cases with a } \\
\text { standardised residual } \\
\text { less or more than } \pm 3\end{array}$}} \\
\hline & \multirow{2}{*}{$\begin{array}{c}\text { Histogram } \\
\text { shows a normal } \\
\text { distribution }\end{array}$} & \multirow{2}{*}{$\begin{array}{l}\text { The Normal P-P Plot } \\
\text { lies in a reasonably } \\
\text { straight line }\end{array}$} & \multirow{2}{*}{$\begin{array}{l}\text { The Scatterplot } \\
\text { shows a rectangular } \\
\text { distribution }\end{array}$} & \multirow{2}{*}{$\begin{array}{l}\text { Durbin- } \\
\text { Watson } \\
\text { values }\end{array}$} & & \\
\hline & & & & & $\begin{array}{c}\text { Case } \\
\text { number }\end{array}$ & $\begin{array}{l}\text { Std. } \\
\text { residual }\end{array}$ \\
\hline TOTAL I & Yes & Yes & Yes & 1.912 & - & - \\
\hline \multirow[t]{2}{*}{ TOTAL IIa } & \multirow[t]{2}{*}{ Yes } & \multirow[t]{2}{*}{ Yes } & \multirow[t]{2}{*}{ Yes } & \multirow[t]{2}{*}{1.968} & 14 & -3.905 \\
\hline & & & & & 196 & -3.154 \\
\hline TOTAL Ilb & Yes & Yes & Yes & 1.871 & 82 & 3.596 \\
\hline TOTAL III & Yes & Yes & Yes & 1.906 & 171 & -3.054 \\
\hline TOTAL IV & Yes & Yes & Yes & 1.956 & - & - \\
\hline TOTAL V & Yes & Yes & Yes & 2.111 & 125 & 3.452 \\
\hline \multirow[t]{5}{*}{ TOTAL VI } & \multirow[t]{5}{*}{ Yes } & \multirow[t]{5}{*}{ Yes } & \multirow[t]{5}{*}{ Yes } & \multirow[t]{5}{*}{1.581} & 160 & 3.302 \\
\hline & & & & & 228 & -3.188 \\
\hline & & & & & 239 & 4.102 \\
\hline & & & & & 280 & 3.892 \\
\hline & & & & & 284 & 3.603 \\
\hline TOTAL VIIa & Yes & Yes & Yes & 2.021 & - & - \\
\hline TOTAL VIIb & Yes & Yes & Yes & 1.971 & - & - \\
\hline \multirow[t]{2}{*}{ TOTAL VIIIa } & \multirow[t]{2}{*}{ Yes } & \multirow[t]{2}{*}{ Yes } & \multirow[t]{2}{*}{ Yes } & \multirow[t]{2}{*}{1.916} & 19 & 3.125 \\
\hline & & & & & 69 & 3.192 \\
\hline TOTAL VIIIlb & Yes & Yes & Yes & 1.740 & 191 & -3.554 \\
\hline TOTAL VIIIIC & Yes & Yes & Yes & 1.870 & 286 & -3.010 \\
\hline
\end{tabular}




\section{TOTAL I}

Histogram

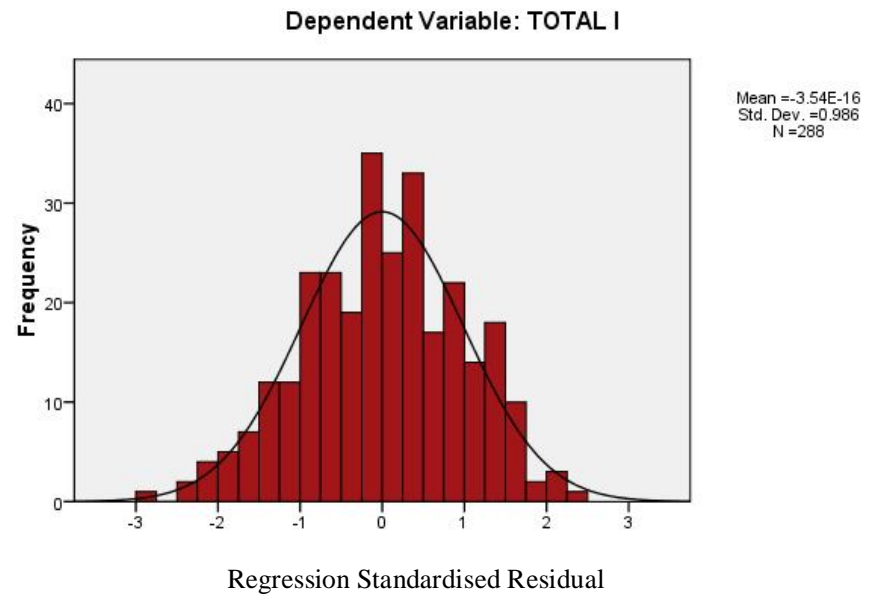

Normal P-P Plot of Regression Standardised Residual

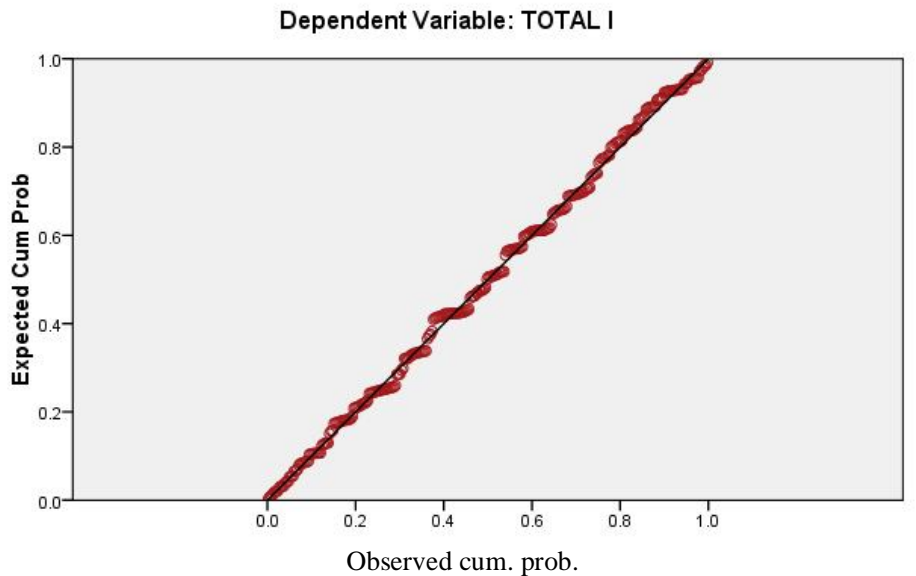

Scatterplot

Dependent Variable: TOTAL I

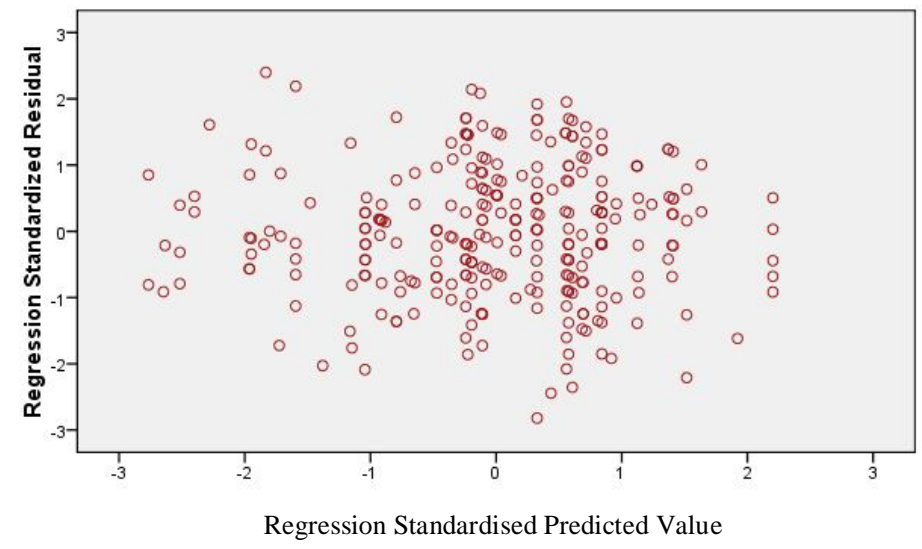




\section{TOTAL IIa}

Histogram

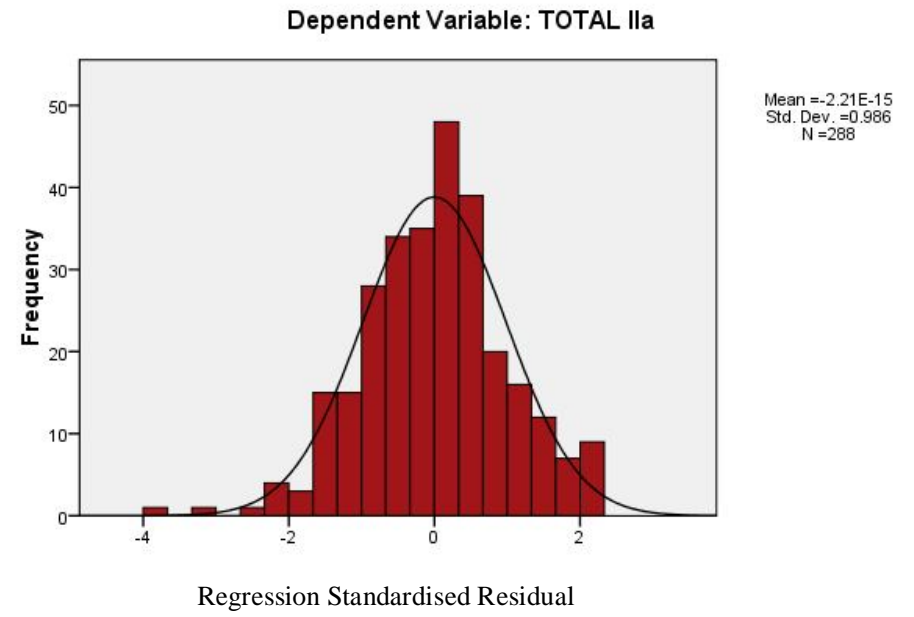

Normal P-P Plot of Regression Standardised Residual

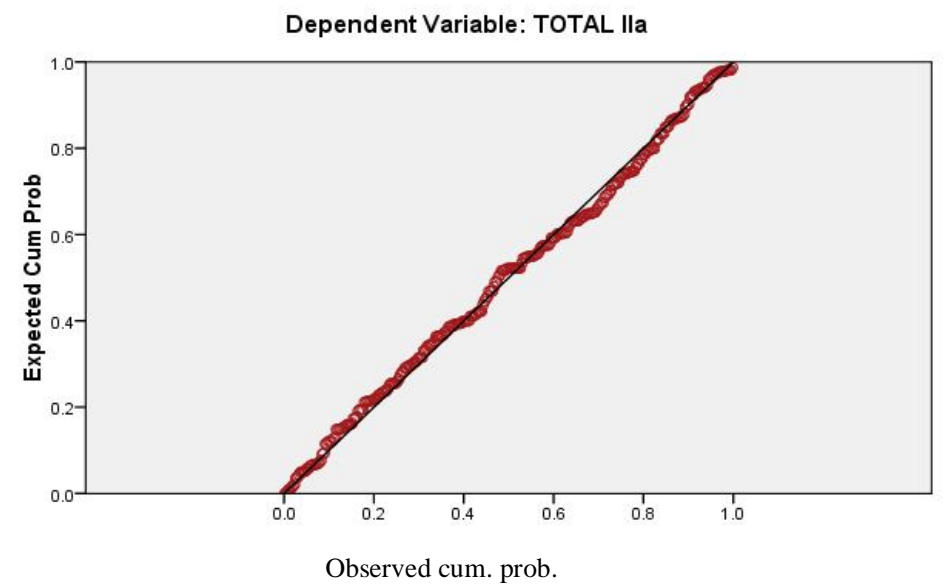

Scatterplot

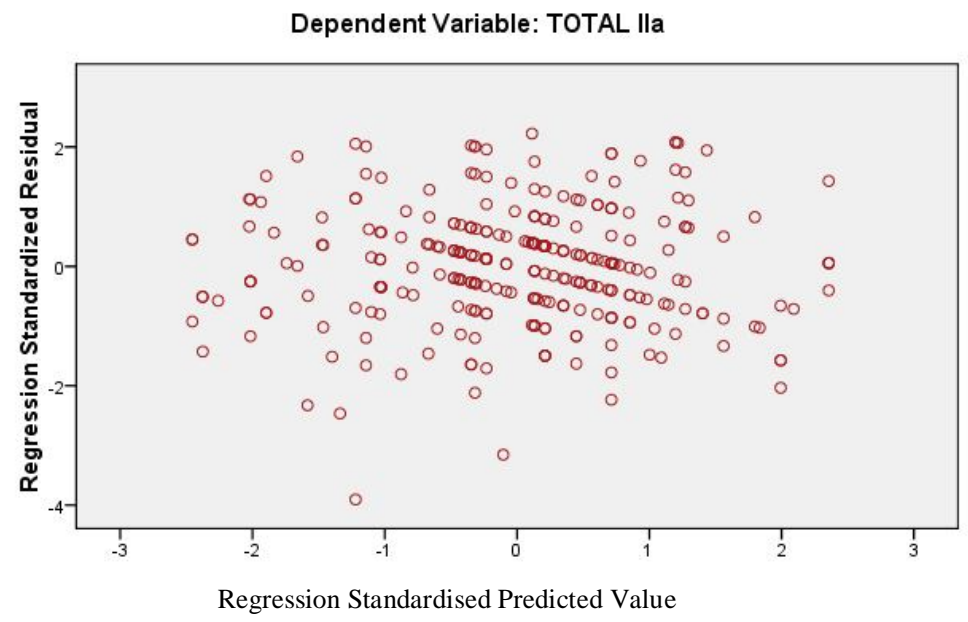




\section{TOTAL IIb}

\section{Histogram}

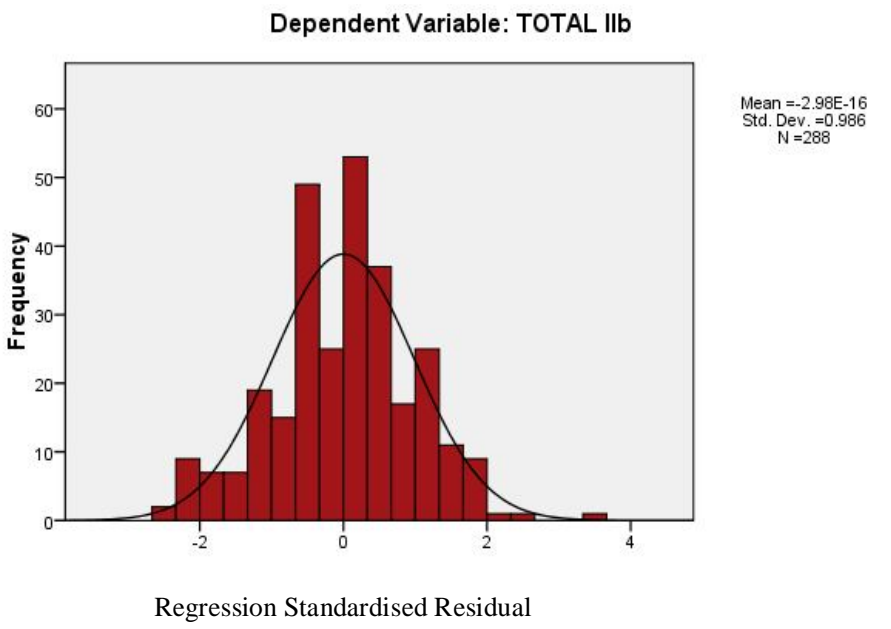

Normal P-P Plot of Regression Standardised Residual

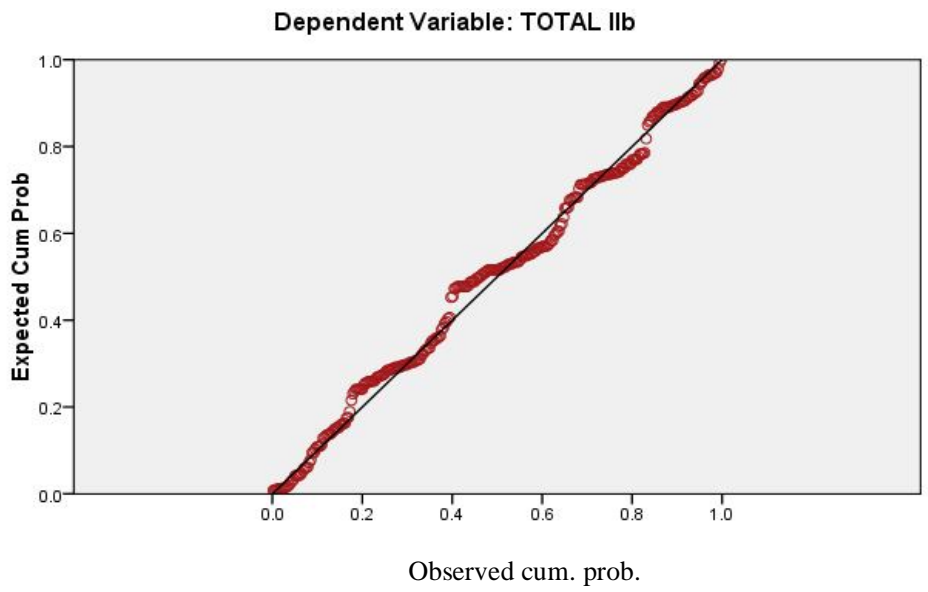

\section{Scatterplot}

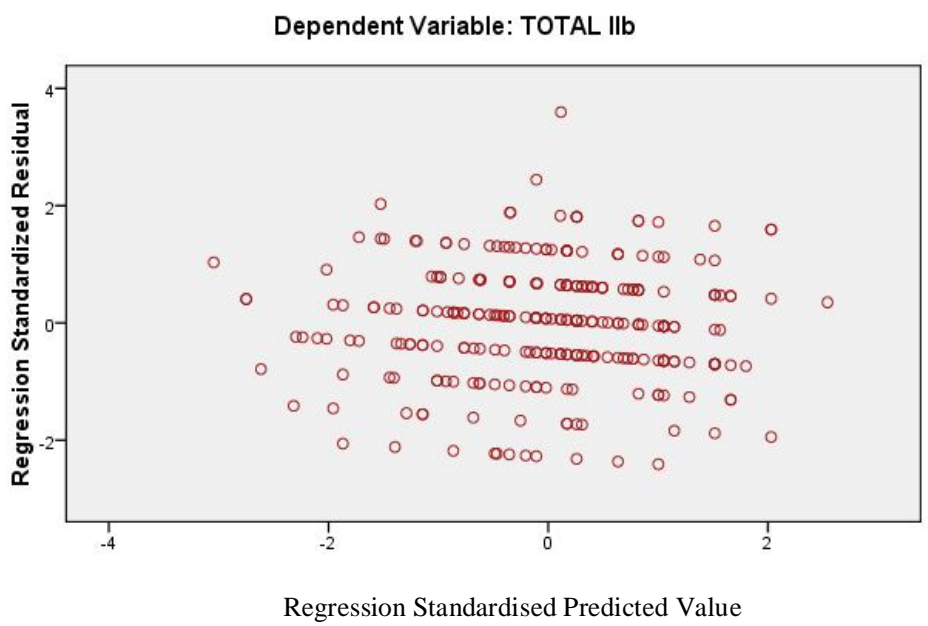




\section{TOTAL III}

Histogram

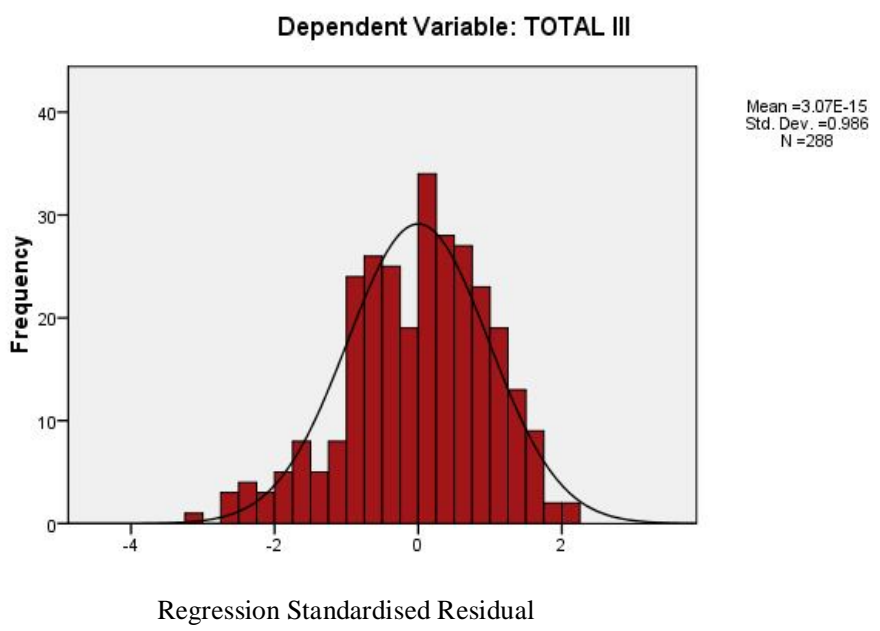

Normal P-P Plot of Regression Standardised Residual

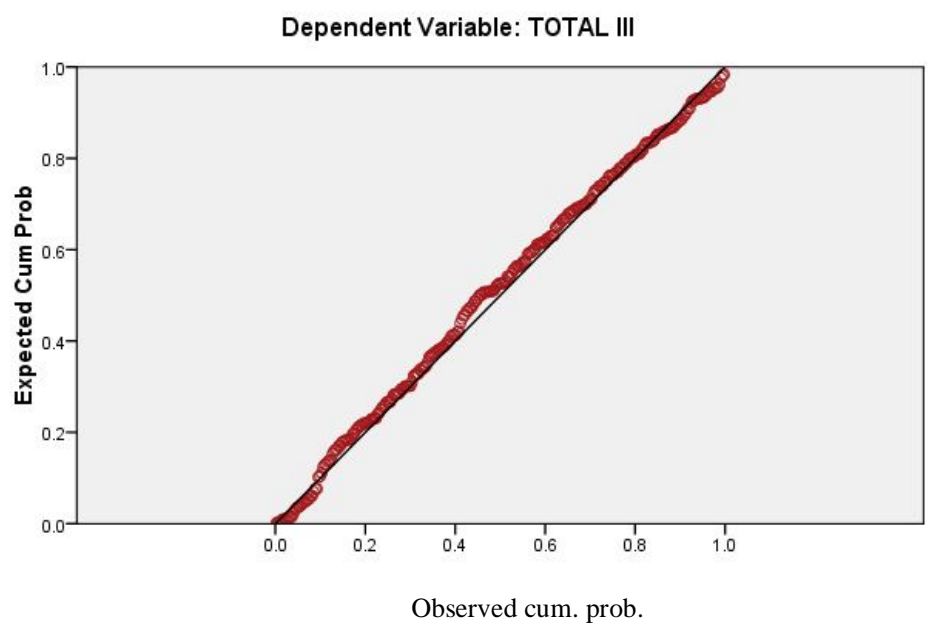

Scatterplot

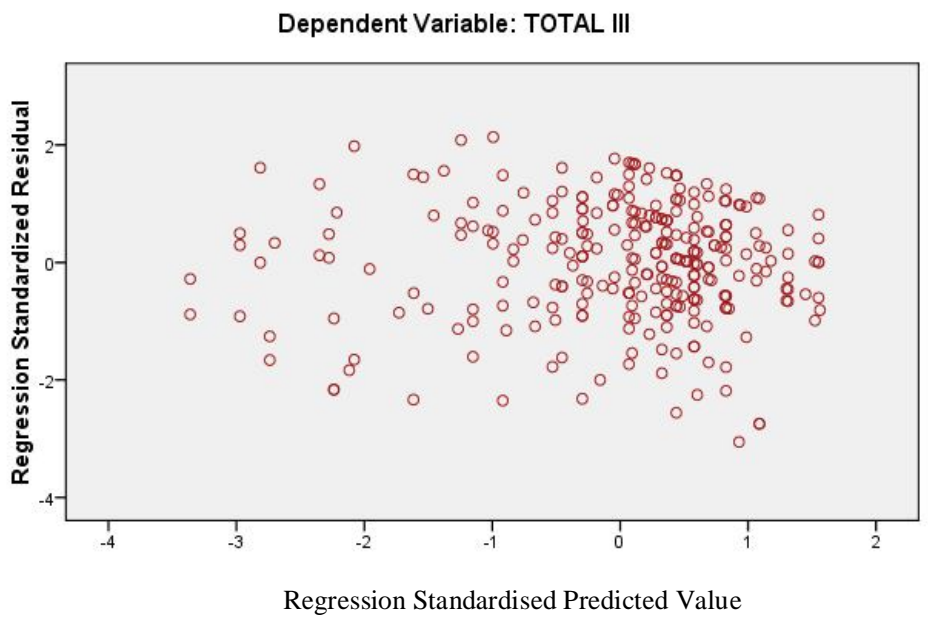




\section{TOTAL IV}

Histogram

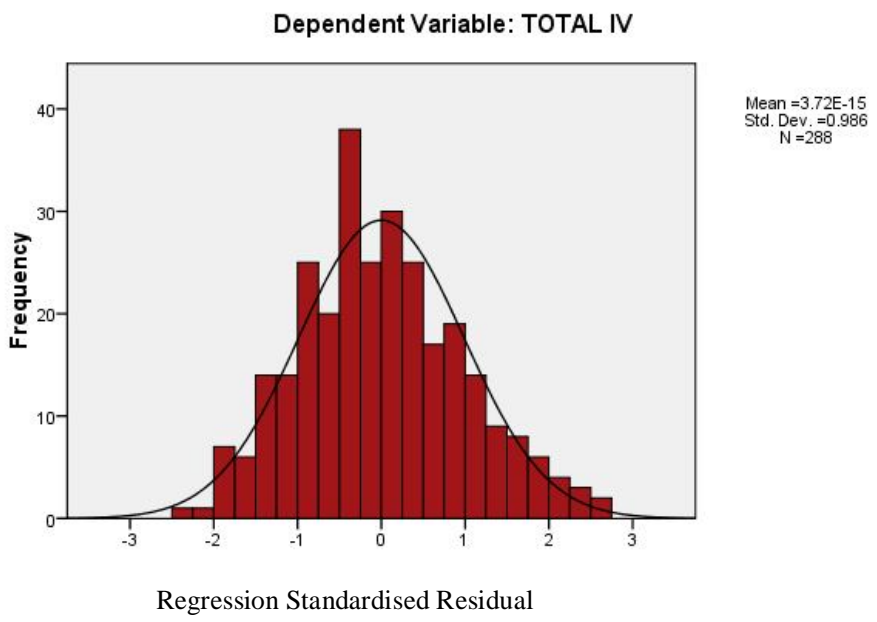

Normal P-P Plot of Regression Standardised Residual

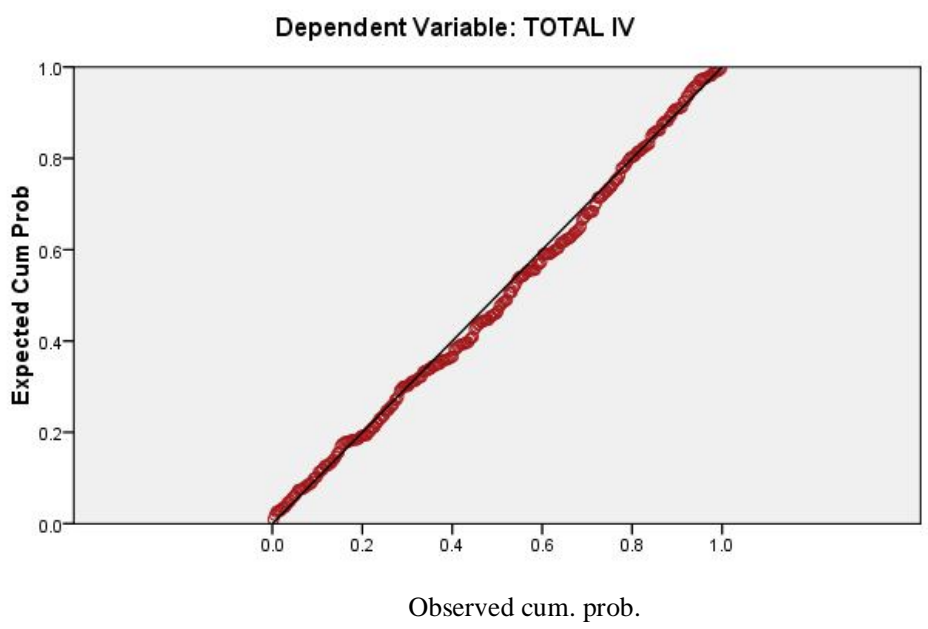

Scatterplot

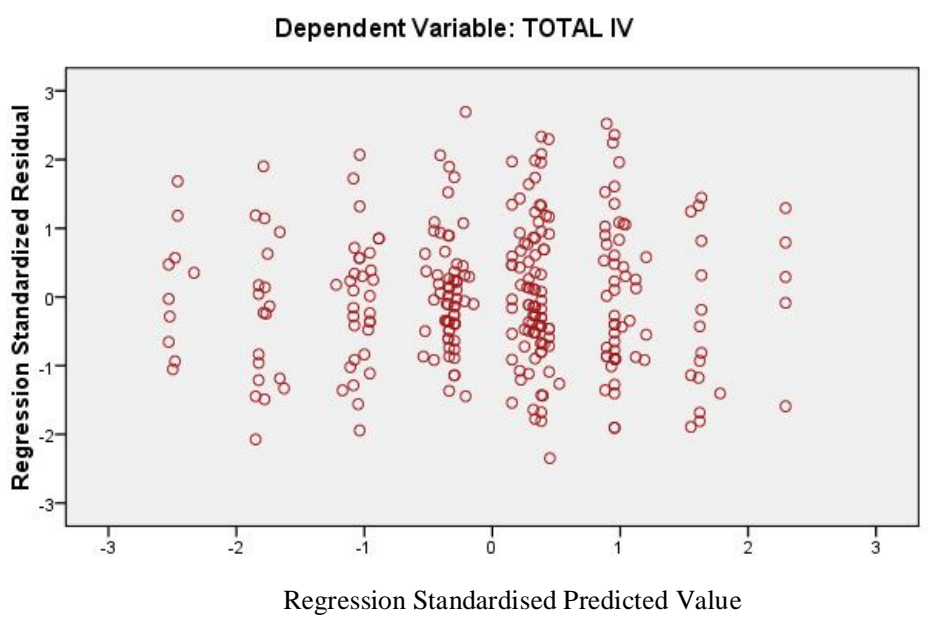




\section{TOTAL V}

Histogram

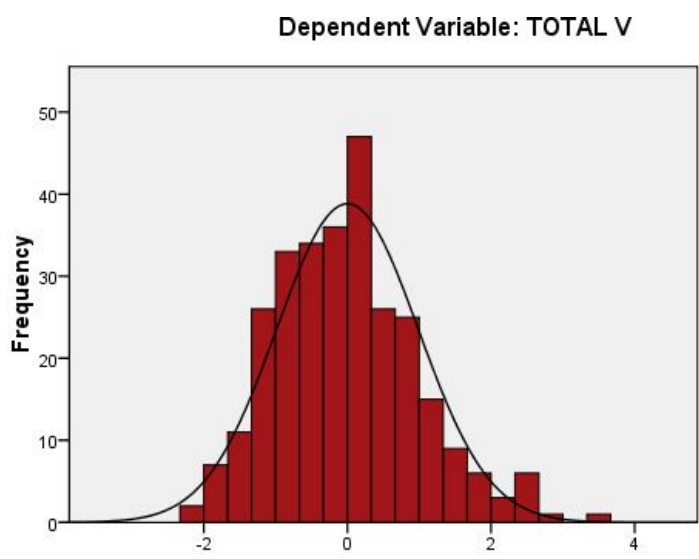

Mean $=6.80 \mathrm{E}-16$
Std. Dev. $=0.986$
$N=288$

Regression Standardised Residual

Normal P-P Plot of Regression Standardised Residual

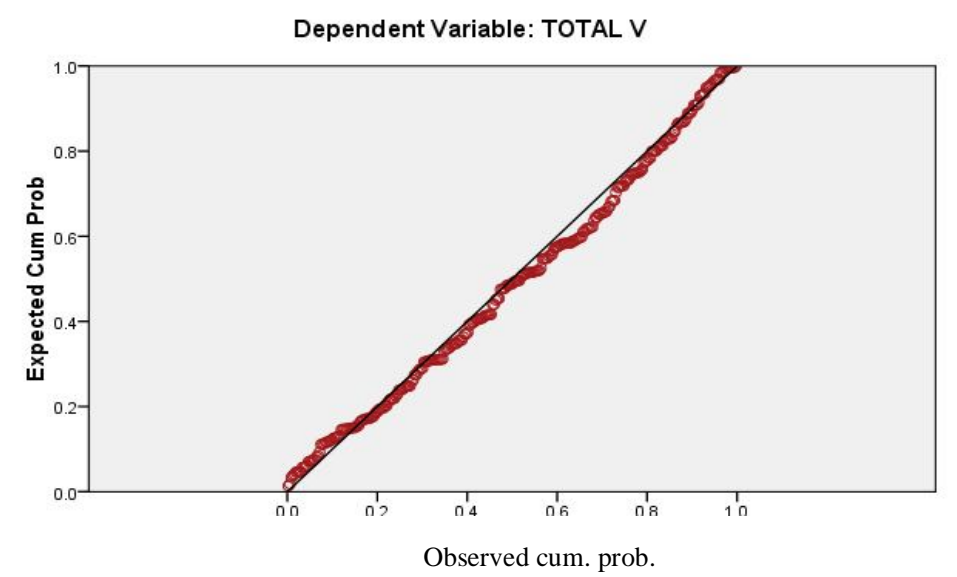

Scatterplot

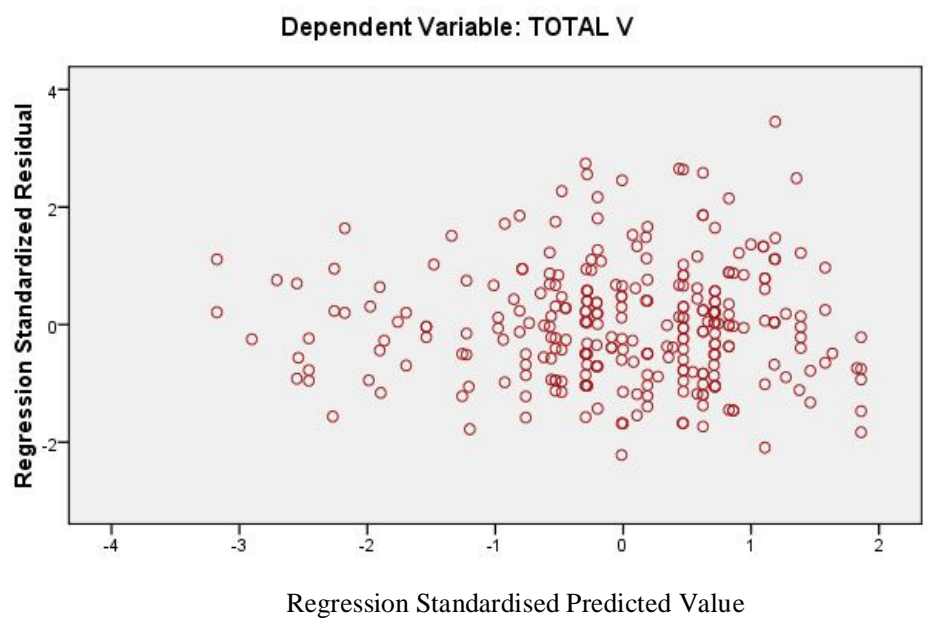




\section{TOTAL VI}

Histogram

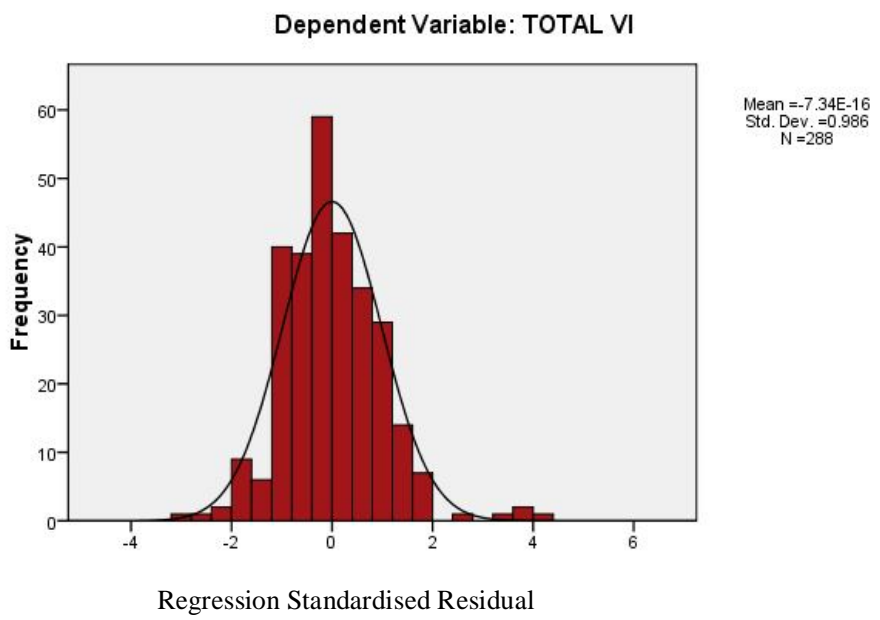

Normal P-P Plot of Regression Standardised Residual

Dependent Variable: TOTAL VI

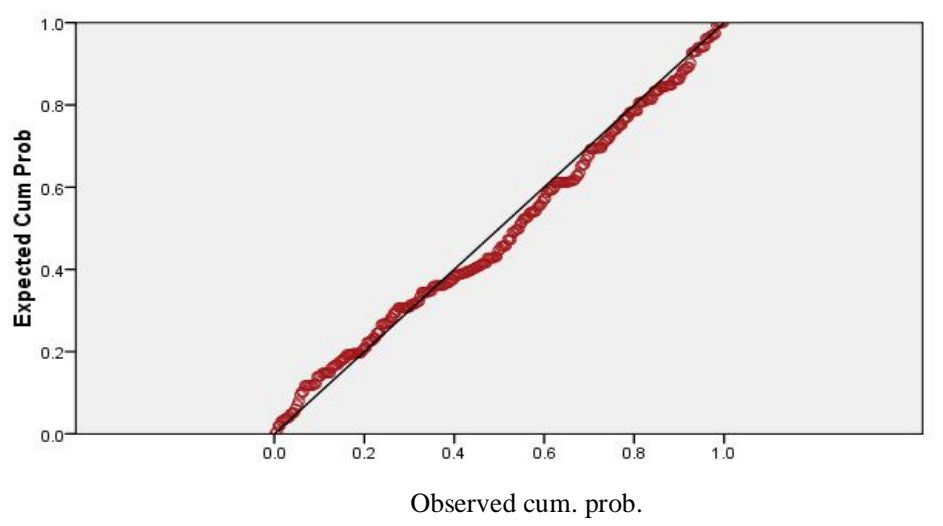

Scatterplot

Dependent Variable: TOTAL VI

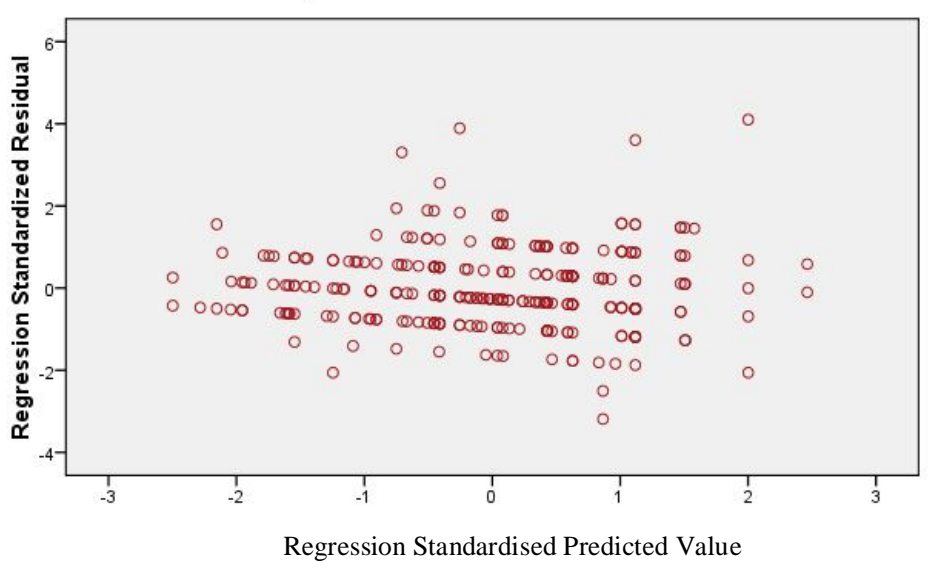




\section{TOTAL VIIa}

Histogram

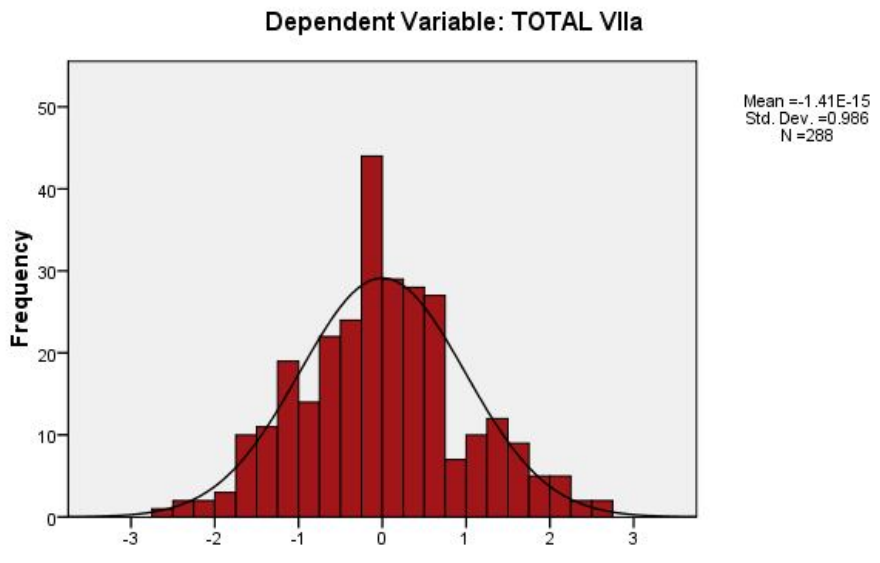

Regression Standardised Residual

Normal P-P Plot of Regression Standardised Residual

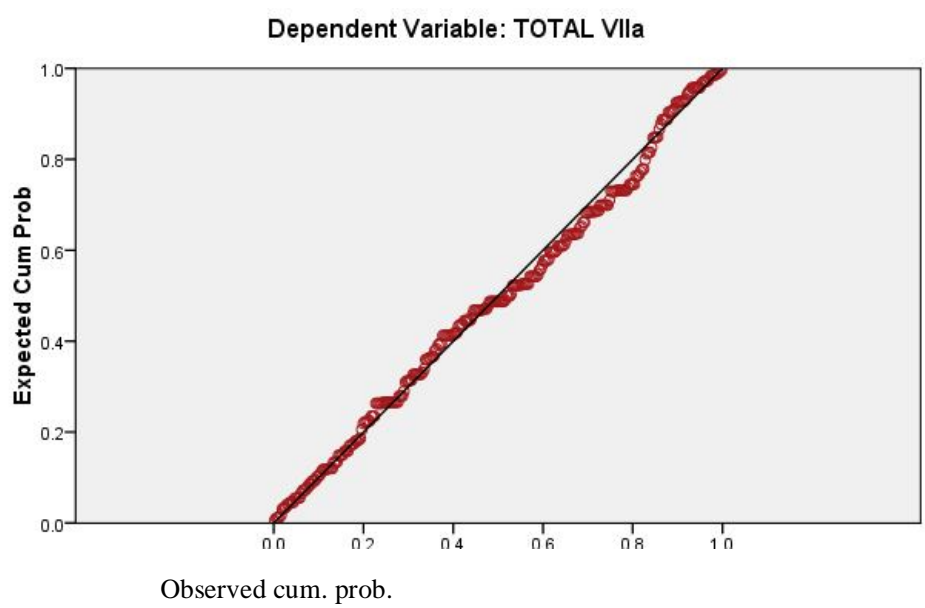

Scatterplot

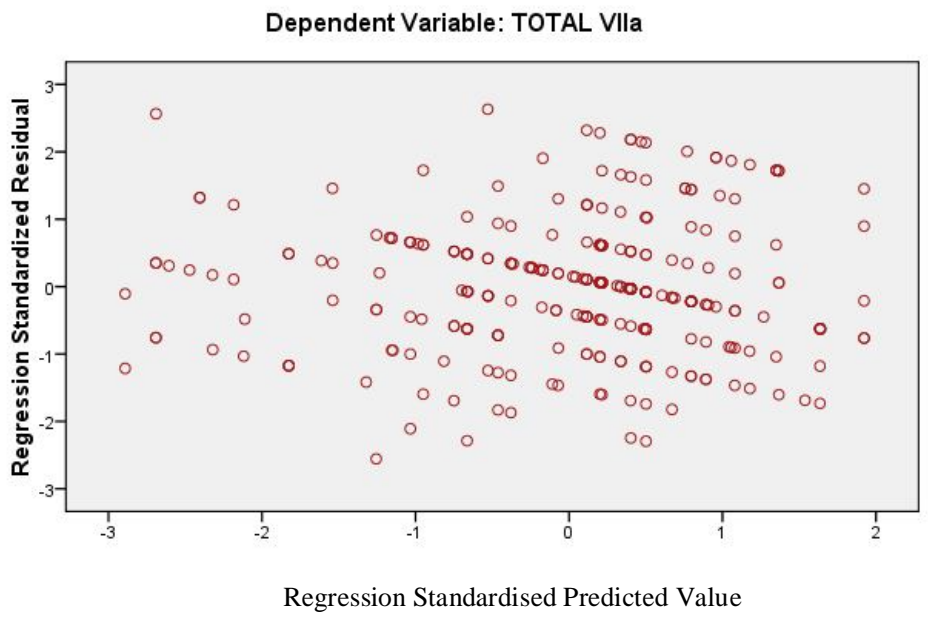




\section{TOTAL VIIb}

Histogram

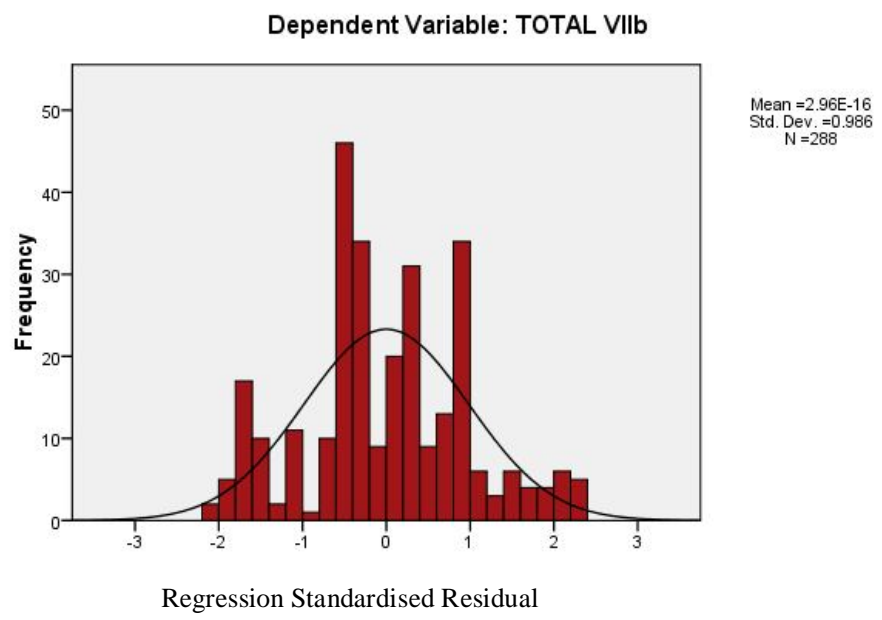

Normal P-P Plot of Regression Standardised Residual

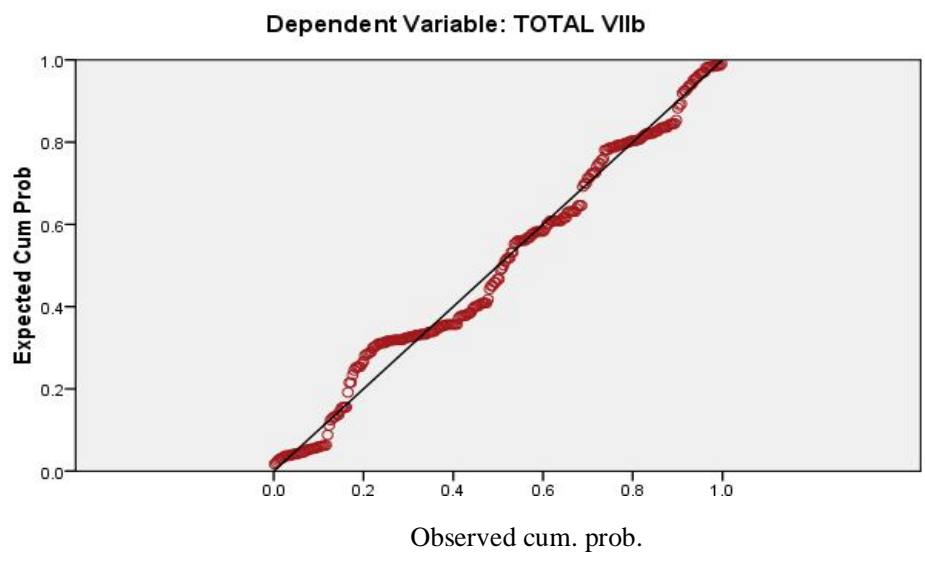

Scatterplot

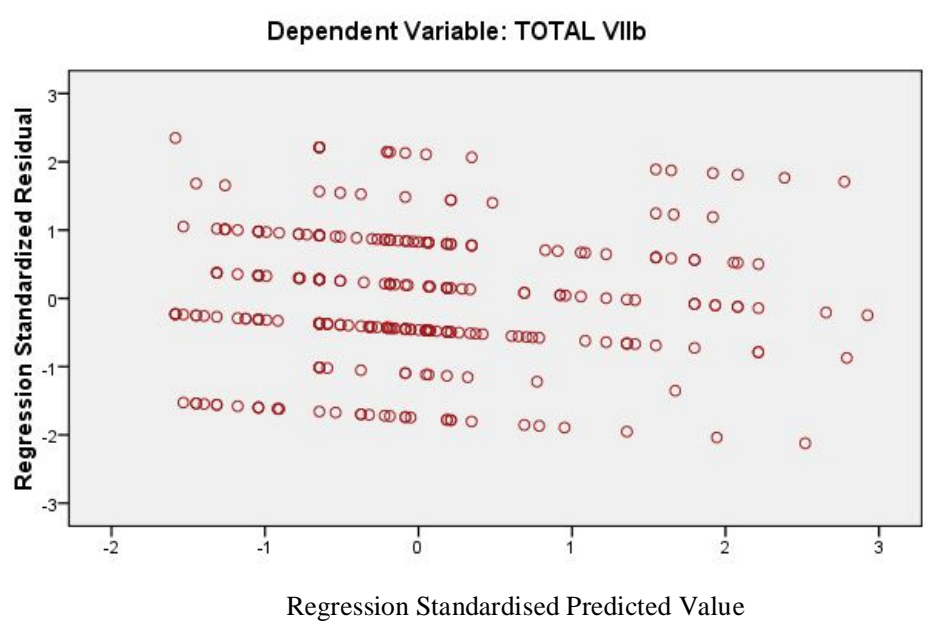




\section{TOTAL VIIIa}

Histogram

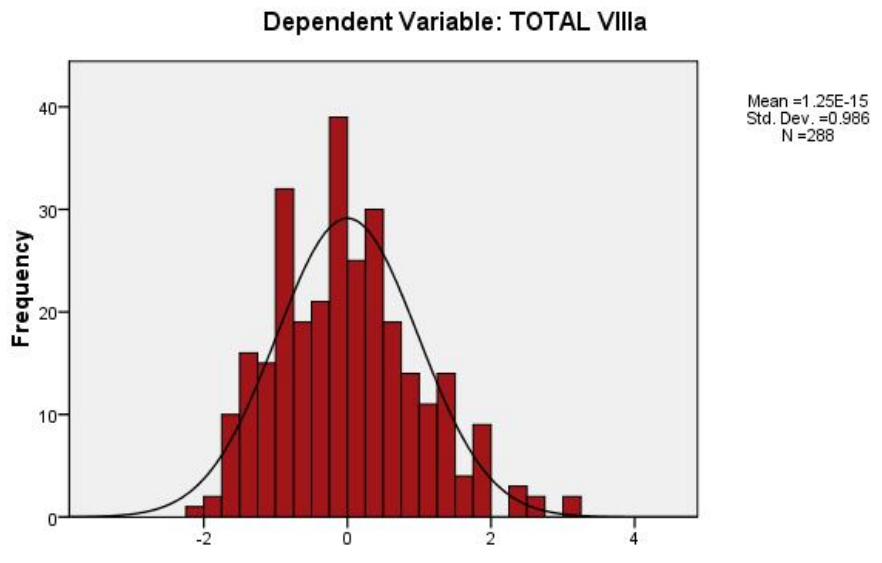

Regression Standardised Residual

Normal P-P Plot of Regression Standardised Residual

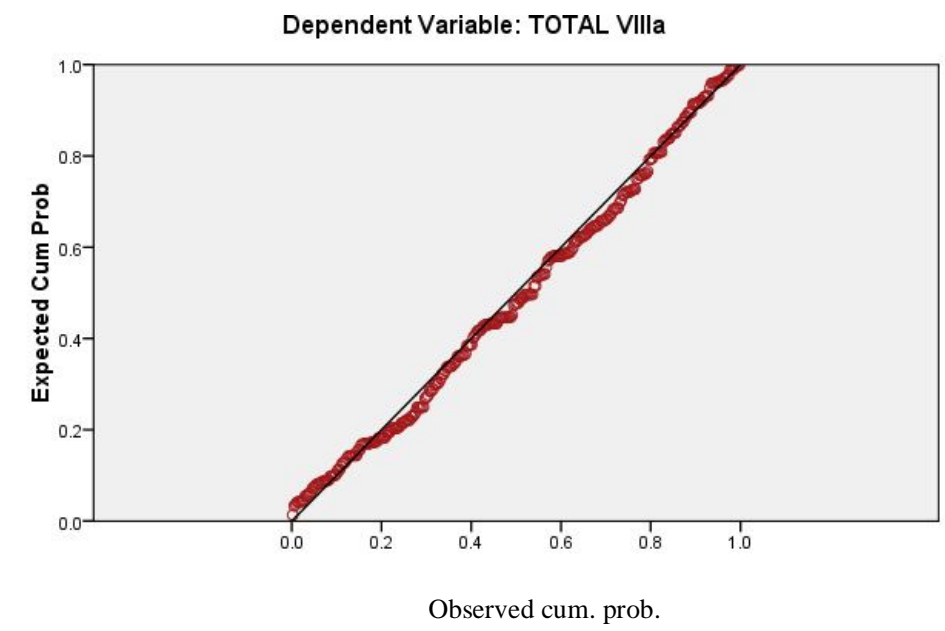

Scatterplot

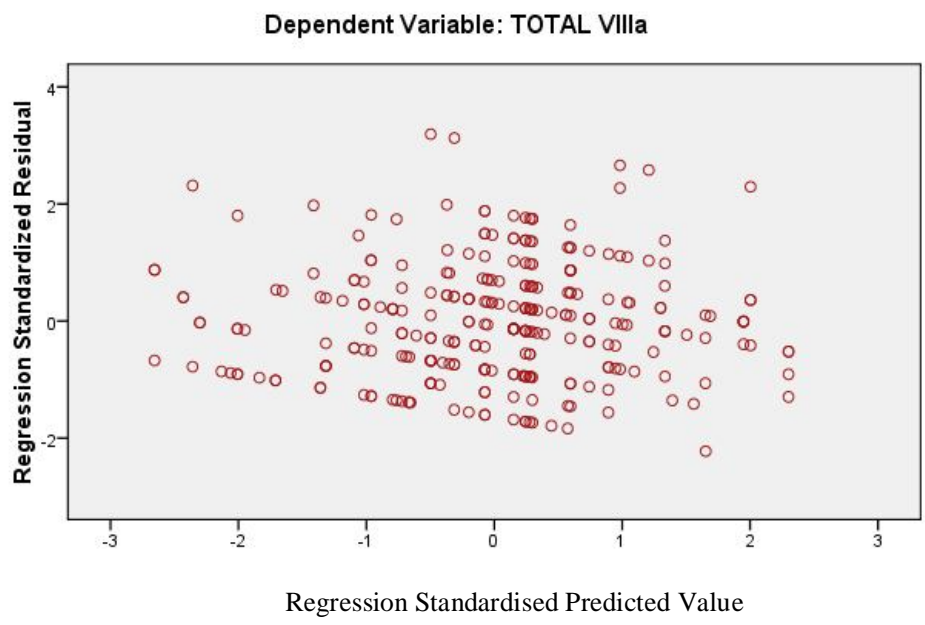




\section{TOTAL VIIIb}

Histogram

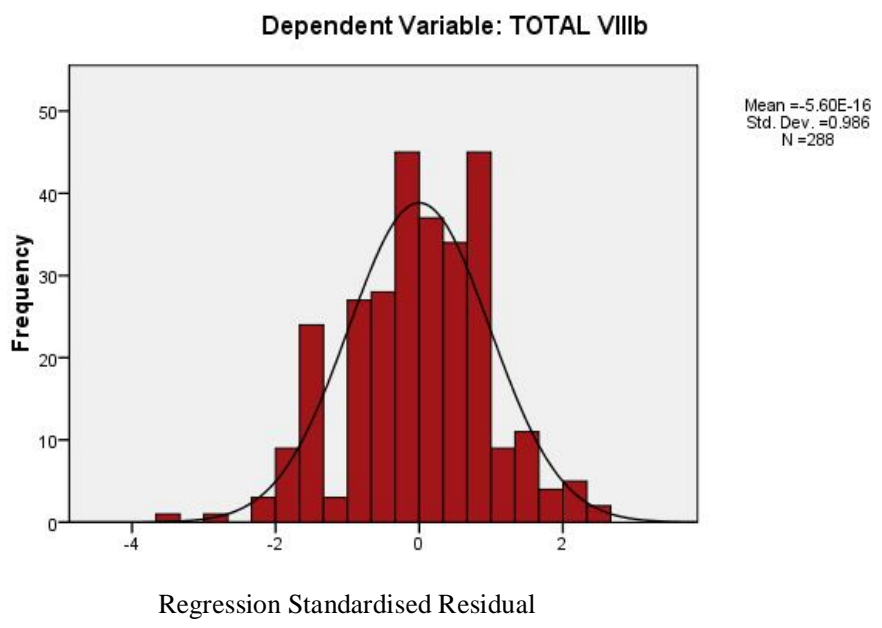

Normal P-P Plot of Regression Standardised Residual

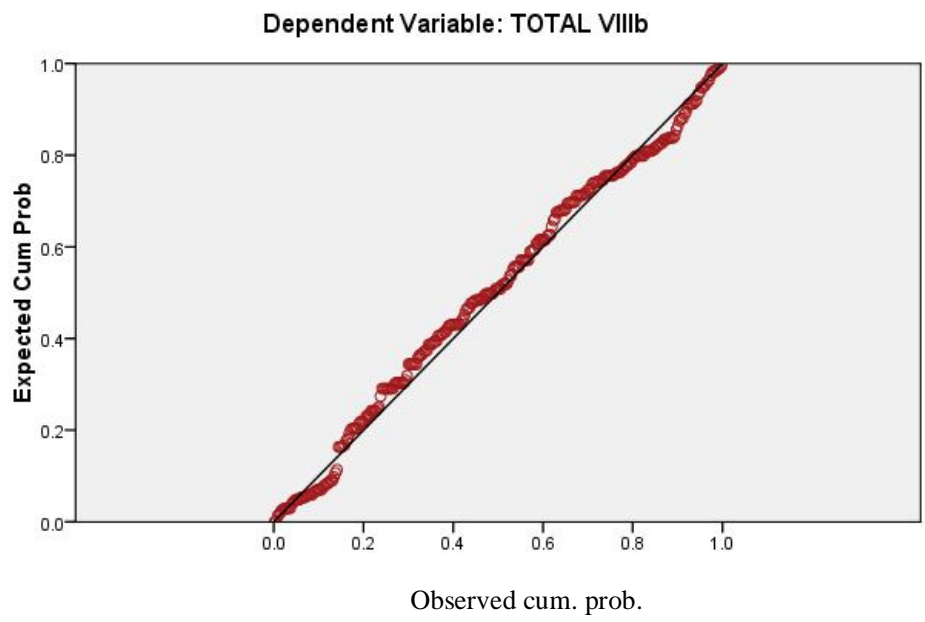

Scatterplot

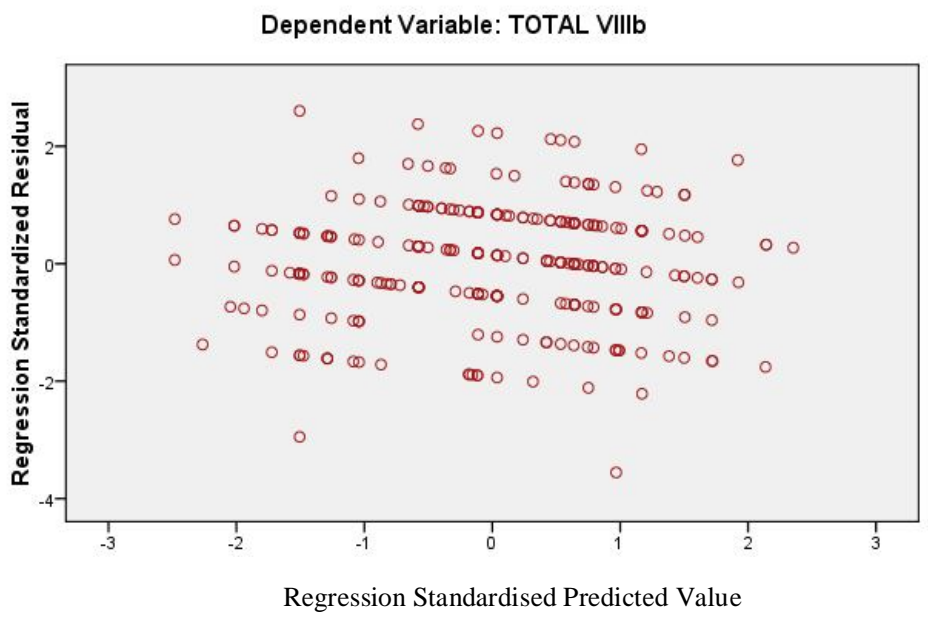




\section{TOTAL VIIIc}

Histogram

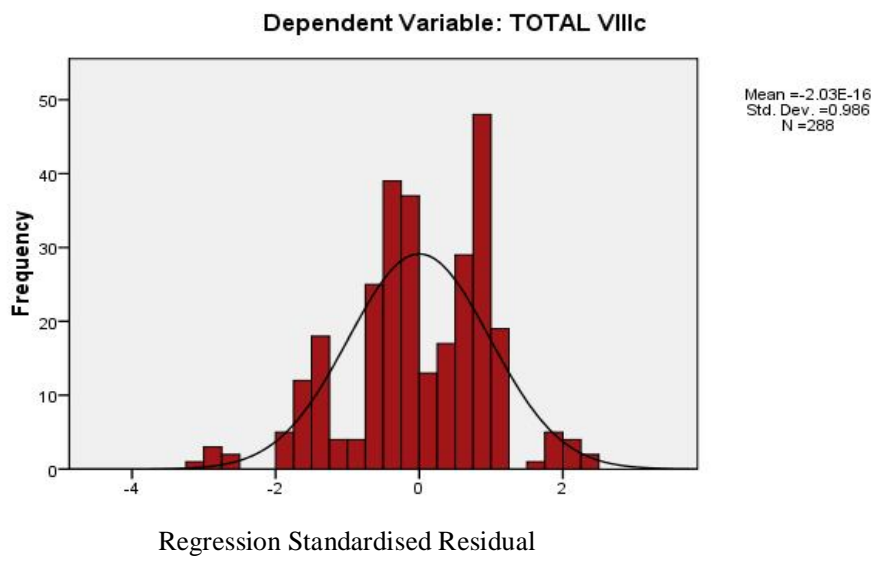

Normal P-P Plot of Regression Standardised Residual

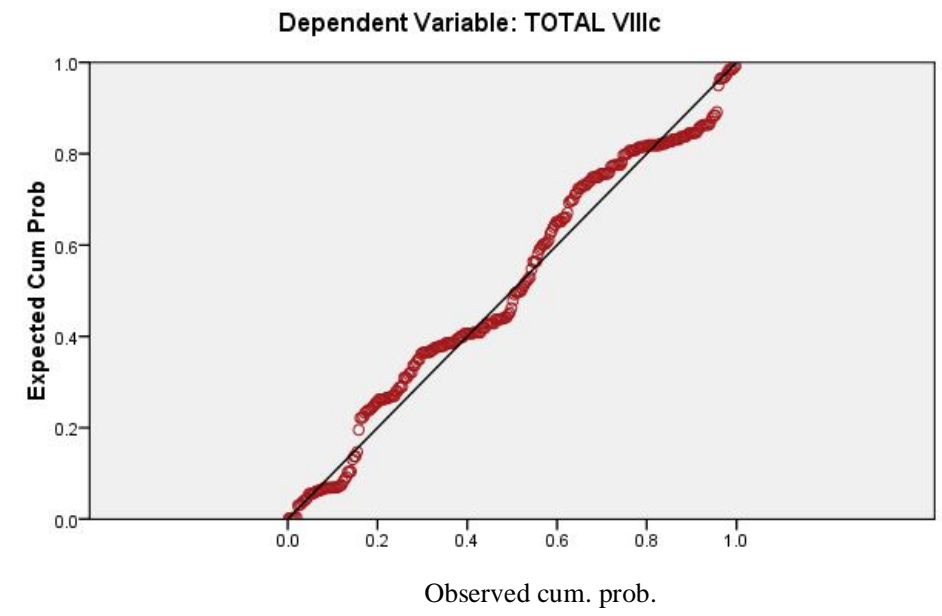

Scatterplot

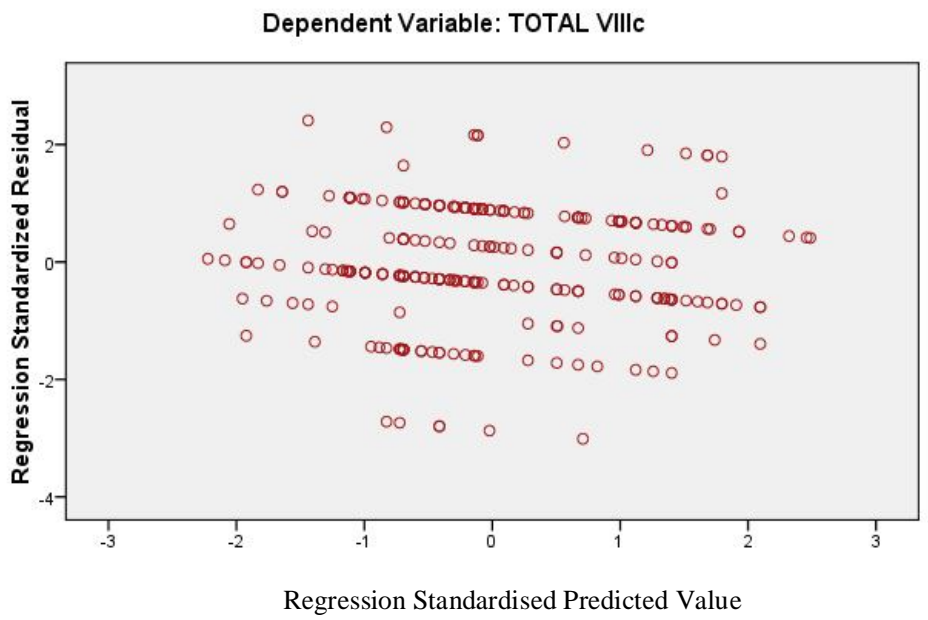




\section{TOTAL all scales (total sum of 12 Scales)}

\section{Histogram}

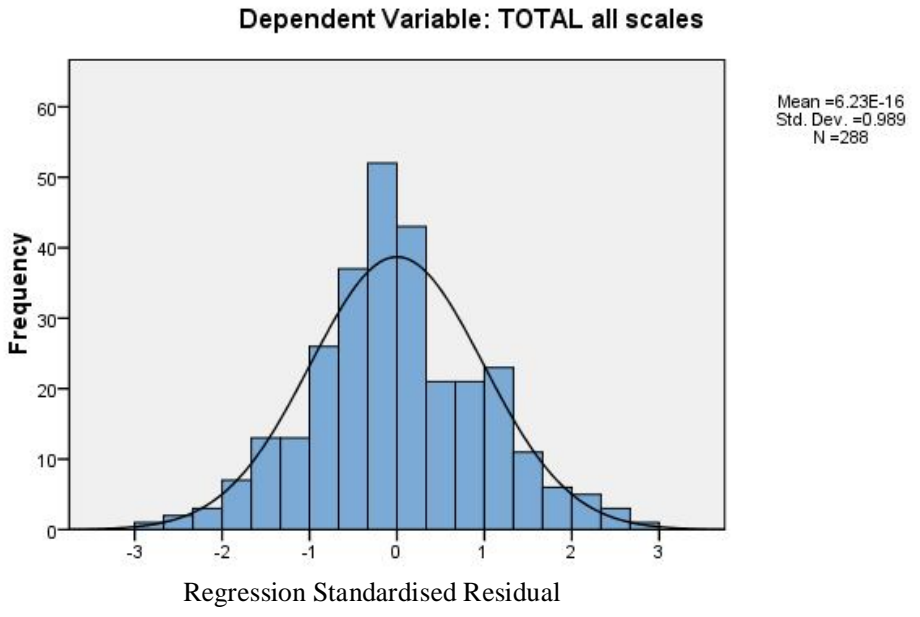

Normal P-P Plot of Regression Standardised Residual

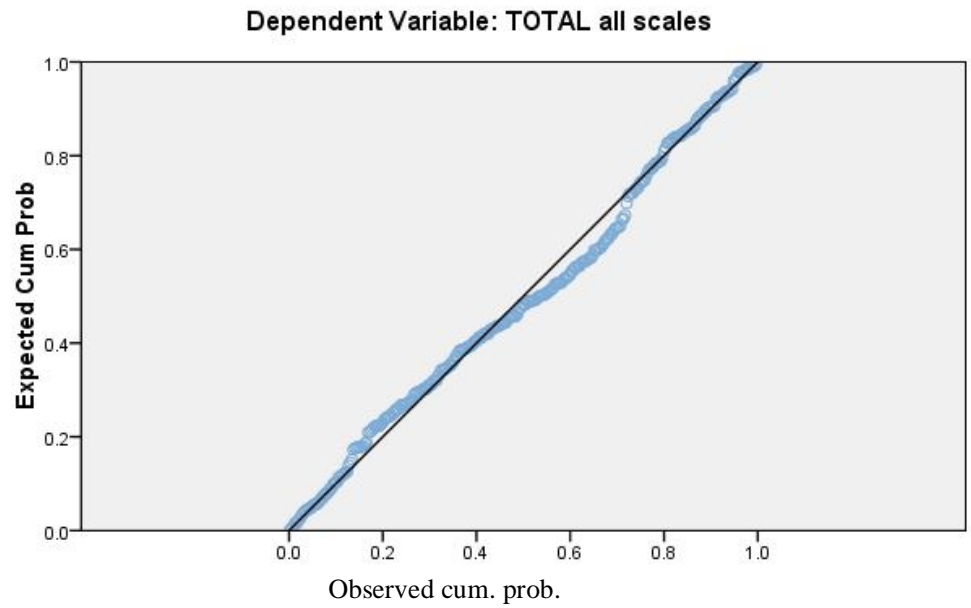

Scatterplot

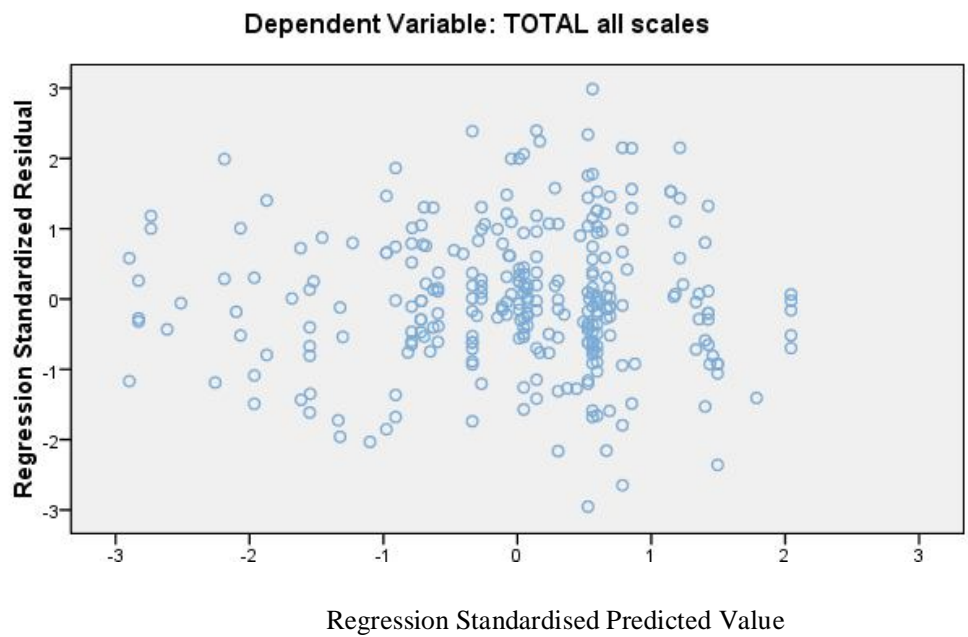




\section{Appendix 7: Multiple regression results}

TOTAL I - Technological skills readiness

\begin{tabular}{|c|c|c|c|c|c|c|c|}
\hline \multirow{2}{*}{ IVs } & \multicolumn{2}{|c|}{$\begin{array}{l}\text { Unstandardised } \\
\text { Coefficients }\end{array}$} & \multirow{2}{*}{$\beta$} & \multirow{2}{*}{$\mathrm{t}$} & \multirow{2}{*}{$\begin{array}{c}p \\
\text { value }\end{array}$} & \multicolumn{2}{|c|}{$\begin{array}{l}\text { 95\% Confidence } \\
\text { Interval for B }\end{array}$} \\
\hline & B & $\begin{array}{l}\text { Std. } \\
\text { Error }\end{array}$ & & & & $\begin{array}{l}\text { Lower } \\
\text { Bound }\end{array}$ & $\begin{array}{l}\text { Upper } \\
\text { Bound }\end{array}$ \\
\hline Age & -2.979 & .324 & -.479 & -9.196 & .000 & -3.617 & -2.341 \\
\hline Education & 2.111 & .500 & .258 & 4.223 & .000 & 1.127 & 3.094 \\
\hline B2athome & -2.947 & .665 & -.209 & -4.433 & .000 & -4.255 & -1.638 \\
\hline B1atwork & -3.422 & .780 & -.208 & -4.387 & .000 & -4.958 & -1.887 \\
\hline Gender & 2.548 & .576 & .208 & 4.425 & .000 & 1.415 & 3.682 \\
\hline Jobtitle1 & -.929 & .761 & -.066 & -1.221 & .223 & -2.427 & .569 \\
\hline Jobtitle2 & .983 & .722 & .082 & 1.362 & .174 & -.438 & 2.405 \\
\hline Jobtitle3 & -.049 & .924 & -.003 & -.053 & .958 & -1.868 & 1.771 \\
\hline
\end{tabular}

TOTAL IIa - Positive online learning style readiness

\begin{tabular}{|l|c|c|c|c|c|c|c|}
\hline \multirow{2}{*}{ IVs } & \multicolumn{2}{|c|}{$\begin{array}{c}\text { Unstandardised } \\
\text { Coefficients }\end{array}$} & \multirow{2}{*}{$\beta$} & \multirow{2}{*}{$\mathbf{t}$} & $\begin{array}{c}\mathbf{p} \\
\text { value }\end{array}$ & \multicolumn{2}{c|}{$\begin{array}{c}\text { 95\% Confidence } \\
\text { Interval for B }\end{array}$} \\
\cline { 2 - 6 } & $\mathbf{B}$ & $\begin{array}{c}\text { Std. } \\
\text { Error }\end{array}$ & & $\begin{array}{c}\text { Lower } \\
\text { Bound }\end{array}$ & $\begin{array}{c}\text { Upper } \\
\text { Bound }\end{array}$ \\
\hline Age & -.969 & .168 & -.351 & -5.781 & .000 & -1.298 & -.639 \\
\hline B2athome & -1.322 & .344 & -.212 & -3.845 & .000 & -1.999 & -.645 \\
\hline Education & .680 & .258 & .188 & 2.633 & .009 & .172 & 1.189 \\
\hline Gender & .443 & .298 & .082 & 1.487 & .138 & -.143 & 1.029 \\
\hline B1atwork & -.535 & .403 & -.073 & -1.326 & .186 & -1.329 & .259 \\
\hline Jobtitle1 & .097 & .393 & .015 & -.245 & .806 & -.678 & .871 \\
\hline Jobtitle2 & .708 & .373 & .133 & 1.896 & .059 & -.027 & 1.443 \\
\hline Jobtitle3 & .515 & .478 & .074 & 1.078 & .282 & -.426 & 1.456 \\
\hline
\end{tabular}

TOTAL IIb - Negative online learning style readiness

\begin{tabular}{|c|c|c|c|c|c|c|c|}
\hline \multirow{2}{*}{ IVs } & \multicolumn{2}{|c|}{$\begin{array}{l}\text { Unstandardised } \\
\text { Coefficients }\end{array}$} & \multirow{2}{*}{$\beta$} & \multirow{2}{*}{$\mathrm{t}$} & \multirow{2}{*}{$\begin{array}{c}p \\
\text { value }\end{array}$} & \multicolumn{2}{|c|}{$\begin{array}{l}\text { 95\% Confidence } \\
\text { Interval for B }\end{array}$} \\
\hline & B & $\begin{array}{l}\text { Std. } \\
\text { Error }\end{array}$ & & & & $\begin{array}{l}\text { Lower } \\
\text { Bound }\end{array}$ & $\begin{array}{l}\text { Upper } \\
\text { Bound }\end{array}$ \\
\hline B1atwork & -.441 & .314 & -.089 & -1.406 & .161 & -1.058 & .176 \\
\hline Age & .106 & .130 & .057 & .817 & .415 & -.150 & .363 \\
\hline Education & -.096 & .201 & -.039 & $\begin{array}{l}-.479 \\
-.49\end{array}$ & .632 & -.492 & .299 \\
\hline B2athome & .136 & .267 & .032 & .510 & .610 & -.390 & .662 \\
\hline Gender & .058 & .231 & .016 & .249 & .803 & -.398 & .513 \\
\hline Jobtitle1 & .183 & .306 & .043 & .597 & .551 & -.419 & .785 \\
\hline Jobtitle2 & .018 & .290 & .005 & .063 & .950 & -.553 & .589 \\
\hline Jobtitle3 & -.225 & .371 & -.047 & -.607 & .545 & -.957 & .506 \\
\hline
\end{tabular}

TOTAL III - Equipment/infrastructure readiness

\begin{tabular}{|c|c|c|c|c|c|c|c|}
\hline \multirow{2}{*}{ IVs } & \multicolumn{2}{|c|}{$\begin{array}{c}\text { Unstandardised } \\
\text { Coefficients }\end{array}$} & \multirow{2}{*}{$\beta$} & \multirow{2}{*}{$t$} & \multirow{2}{*}{$\begin{array}{c}p \\
\text { value }\end{array}$} & \multicolumn{2}{|c|}{$\begin{array}{l}95 \% \text { Confidence } \\
\text { Interval for B }\end{array}$} \\
\hline & B & $\begin{array}{l}\text { Std. } \\
\text { Error }\end{array}$ & & & & $\begin{array}{l}\text { Lower } \\
\text { Bound }\end{array}$ & $\begin{array}{l}\text { Upper } \\
\text { Bound }\end{array}$ \\
\hline B1atwork & -5.411 & .916 & -.321 & -5.908 & .000 & -7.214 & -3.608 \\
\hline Age & -1.847 & .380 & -.290 & -4.856 & .000 & -2.596 & -1.098 \\
\hline Education & 2.182 & .587 & .261 & 3.719 & .000 & 1.027 & 3.337 \\
\hline Gender & 1.375 & .676 & .109 & 2.033 & .043 & .044 & 2.706 \\
\hline B2athome & -.708 & .780 & -.049 & -.907 & .365 & -2.244 & .829 \\
\hline Jobtitle1 & -1.154 & .893 & -.079 & -1.292 & .197 & -2.913 & .604 \\
\hline Jobtitle2 & -.740 & .848 & -.060 & -.873 & .383 & -2.409 & .928 \\
\hline Jobtitle3 & -.115 & 1.085 & -.007 & -.106 & .916 & -2.251 & 2.021 \\
\hline
\end{tabular}

TOTAL IV - Attitude readiness

\begin{tabular}{|l|c|c|c|c|c|c|c|}
\hline \multirow{2}{*}{ IVs } & \multicolumn{2}{|c|}{$\begin{array}{c}\text { Unstandardised } \\
\text { Coefficients }\end{array}$} & \multirow{2}{*}{$\beta$} & \multirow{2}{*}{$\mathbf{f}$} & $\begin{array}{c}\mathbf{p} \\
\text { value }\end{array}$ & \multicolumn{2}{c|}{$\begin{array}{c}\text { 95\% Confidence } \\
\text { Interval for B }\end{array}$} \\
\cline { 2 - 6 } & $\mathbf{B}$ & $\begin{array}{c}\text { Std. } \\
\text { Error }\end{array}$ & & $\begin{array}{c}\text { Lower } \\
\text { Bound }\end{array}$ & $\begin{array}{c}\text { Upper } \\
\text { Bound }\end{array}$ \\
\hline Age & -3.601 & .612 & -.349 & -5.883 & .000 & -4.806 & -2.396 \\
\hline Education & 3.304 & .944 & .244 & 3.498 & .001 & 1.445 & 5.163 \\
\hline Gender & 3.262 & 1.088 & .161 & 2.998 & .003 & 1.120 & 5.404 \\
\hline B2athome & -3.201 & 1.256 & -.137 & -2.548 & .011 & -5.673 & -.728 \\
\hline B1atwork & -3.422 & 1.474 & -.125 & -2.321 & .021 & -6.324 & -.520 \\
\hline Jobtitle1 & .238 & 1.438 & .010 & .165 & .869 & -2.592 & 3.068 \\
\hline Jobtitle2 & 3.022 & 1.364 & .152 & 2.215 & .028 & .336 & 5.707 \\
\hline Jobtitle3 & .922 & 1.747 & .035 & .528 & .598 & -2.516 & 4.361 \\
\hline
\end{tabular}


TOTAL V - Human resource readiness

\begin{tabular}{|l|c|c|c|c|c|c|c|}
\hline \multirow{2}{*}{ IVs } & \multicolumn{2}{|c|}{$\begin{array}{c}\text { Unstandardised } \\
\text { Coefficients }\end{array}$} & \multirow{2}{*}{$\beta$} & \multirow{2}{*}{$\mathbf{t}$} & $\begin{array}{c}\mathbf{p} \\
\text { value }\end{array}$ & \multicolumn{2}{c|}{$\begin{array}{c}95 \% \text { Confidence } \\
\text { Interval for B }\end{array}$} \\
\cline { 2 - 6 } & $\mathbf{B}$ & $\begin{array}{c}\text { Std. } \\
\text { Error }\end{array}$ & & & $\begin{array}{c}\text { Lower } \\
\text { Bound }\end{array}$ & $\begin{array}{c}\text { Upper } \\
\text { Bound }\end{array}$ \\
\hline Education & 1.888 & .658 & .220 & 2.867 & .004 & .592 & 3.184 \\
\hline B1atwork & -3.489 & 1.028 & -.202 & -3.394 & .001 & -5.512 & -1.465 \\
\hline Gender & 2.057 & .759 & .160 & 2.711 & .007 & .563 & 3.550 \\
\hline Age & -.578 & .427 & -.089 & -1.354 & .177 & -1.418 & .262 \\
\hline B2athome & -.971 & .876 & -.066 & -1.108 & .269 & -2.695 & .753 \\
\hline Jobtitle1 & -.193 & 1.002 & -.013 & -.192 & .848 & -2.166 & 1.780 \\
\hline Jobtitle2 & -.509 & .951 & -.040 & -.535 & .593 & -2.381 & 1.364 \\
\hline Jobtitle3 & -.748 & 1.218 & -.045 & -.614 & .540 & -3.145 & 1.649 \\
\hline
\end{tabular}

TOTAL VI - Environmental readiness

\begin{tabular}{|c|c|c|c|c|c|c|c|}
\hline \multirow{2}{*}{ IVs } & \multicolumn{2}{|c|}{$\begin{array}{l}\text { Unstandardised } \\
\text { Coefficients }\end{array}$} & \multirow{2}{*}{$\beta$} & \multirow{2}{*}{$t$} & \multirow{2}{*}{$\begin{array}{c}p \\
\text { value }\end{array}$} & \multicolumn{2}{|c|}{$\begin{array}{l}\text { 95\% Confidence } \\
\text { Interval for B }\end{array}$} \\
\hline & B & $\begin{array}{l}\text { Std. } \\
\text { Error }\end{array}$ & & & & $\begin{array}{l}\text { Lower } \\
\text { Bound }\end{array}$ & $\begin{array}{l}\text { Upper } \\
\text { Bound }\end{array}$ \\
\hline Education & .271 & .173 & .126 & 1.567 & .118 & -.070 & .612 \\
\hline Gender & .319 & .199 & .099 & 1.599 & .111 & -.074 & .711 \\
\hline B2athome & .349 & .230 & .094 & 1.515 & .131 & -.104 & .802 \\
\hline Age & -.152 & .112 & -.093 & -1.356 & .176 & -.373 & .069 \\
\hline B1atwork & -.218 & .270 & -.050 & -.807 & .420 & -.750 & .314 \\
\hline Jobtitle1 & -.061 & .263 & -.016 & -.233 & .816 & -.580 & .457 \\
\hline Jobtitle2 & .151 & .250 & .048 & .603 & .547 & -.341 & .643 \\
\hline Jobtitle3 & .293 & .320 & .070 & .914 & .361 & -.337 & .922 \\
\hline
\end{tabular}

\section{TOTAL VIIa - Positive cultural readiness}

\begin{tabular}{|c|c|c|c|c|c|c|c|}
\hline \multirow{2}{*}{ IVs } & \multicolumn{2}{|c|}{$\begin{array}{l}\text { Unstandardised } \\
\text { Coefficients }\end{array}$} & \multirow{2}{*}{$\beta$} & \multirow{2}{*}{$\mathrm{t}$} & \multirow{2}{*}{$\begin{array}{c}p \\
\text { value }\end{array}$} & \multicolumn{2}{|c|}{$\begin{array}{l}\text { 95\% Confidence } \\
\text { Interval for B }\end{array}$} \\
\hline & B & $\begin{array}{l}\text { Std. } \\
\text { Error }\end{array}$ & & & & $\begin{array}{l}\text { Lower } \\
\text { Bound }\end{array}$ & $\begin{array}{l}\text { Upper } \\
\text { Bound }\end{array}$ \\
\hline Education & .753 & .214 & .259 & 3.518 & .001 & .332 & 1.174 \\
\hline B1atwork & -1.441 & .334 & -.246 & -4.315 & .000 & -2.098 & -.784 \\
\hline Age & -.248 & .139 & -.112 & -1.791 & .074 & -.521 & .025 \\
\hline B2athome & -.483 & .285 & -.097 & -1.697 & .091 & -1.043 & .077 \\
\hline gender & .250 & .247 & .057 & 1.013 & .312 & -.235 & .735 \\
\hline Jobtitle1 & -.175 & .326 & -.035 & -.538 & .591 & -.816 & .466 \\
\hline Jobtitle2 & .086 & .309 & .020 & .279 & .780 & $\begin{array}{l}-.522 \\
\end{array}$ & .695 \\
\hline Jobtitle3 & -.313 & .396 & -.056 & -.790 & .430 & -1.092 & .466 \\
\hline
\end{tabular}

\section{TOTAL VIIb - Negative cultural readiness}

\begin{tabular}{|c|c|c|c|c|c|c|c|}
\hline \multirow{2}{*}{ IVs } & \multicolumn{2}{|c|}{$\begin{array}{l}\text { Unstandardised } \\
\text { Coefficients }\end{array}$} & \multirow{2}{*}{$ß$} & \multirow{2}{*}{$t$} & \multirow{2}{*}{$\begin{array}{c}p \\
\text { value }\end{array}$} & \multicolumn{2}{|c|}{$\begin{array}{l}\text { 95\% Confidence } \\
\text { Interval for B }\end{array}$} \\
\hline & B & $\begin{array}{l}\text { Std. } \\
\text { Error }\end{array}$ & & & & $\begin{array}{l}\text { Lower } \\
\text { Bound }\end{array}$ & $\begin{array}{l}\text { Upper } \\
\text { Bound }\end{array}$ \\
\hline B2athome & -.394 & .244 & -.101 & -1.614 & .108 & -.875 & .087 \\
\hline gender & -.195 & .212 & -.057 & -.921 & .358 & -612 & .222 \\
\hline Education & .062 & .184 & .027 & .336 & .737 & -.300 & .423 \\
\hline Age & -.031 & .119 & -.018 & -.258 & .796 & -.265 & .204 \\
\hline B1atwork & .012 & .287 & .003 & .041 & .967 & -.553 & .576 \\
\hline Jobtitle1 & .280 & .280 & .071 & .999 & .318 & -.271 & .830 \\
\hline Jobtitle2 & .090 & .265 & .027 & .340 & .734 & $\begin{array}{l}-.432 \\
\end{array}$ & .613 \\
\hline Jobtitle3 & .252 & .340 & .058 & .742 & .459 & -.417 & .921 \\
\hline
\end{tabular}

TOTAL VIIIa - Financial affordability readiness

\begin{tabular}{|c|c|c|c|c|c|c|c|}
\hline \multirow{2}{*}{ IVs } & \multicolumn{2}{|c|}{$\begin{array}{l}\text { Unstandardised } \\
\text { Coefficients }\end{array}$} & \multirow{2}{*}{$\beta$} & \multirow{2}{*}{$\mathrm{t}$} & \multirow{2}{*}{$\begin{array}{c}p \\
\text { value }\end{array}$} & \multicolumn{2}{|c|}{$\begin{array}{l}\text { 95\% Confidence } \\
\text { Interval for B }\end{array}$} \\
\hline & B & $\begin{array}{l}\text { Std. } \\
\text { Error }\end{array}$ & & & & $\begin{array}{l}\text { Lower } \\
\text { Bound }\end{array}$ & $\begin{array}{l}\text { Upper } \\
\text { Bound }\end{array}$ \\
\hline B1atwork & -1.245 & .478 & -.156 & -2.606 & .010 & -2.186 & -.305 \\
\hline Education & .602 & .306 & .152 & 1.968 & .050 & .000 & 1.205 \\
\hline B2athome & -.982 & .407 & -.144 & -2.413 & .016 & -1.784 & -.181 \\
\hline Age & -.277 & .198 & -.091 & -1.394 & .165 & -.667 & .114 \\
\hline Gender & -.327 & .353 & -.055 & -.928 & .354 & -1.022 & .367 \\
\hline Jobtitle1 & .209 & .466 & .030 & .449 & .654 & -.708 & 1.126 \\
\hline Jobtitle2 & .296 & .442 & .051 & .669 & .504 & -.575 & 1.166 \\
\hline Jobtitle3 & -.273 & .566 & -.036 & -.481 & .631 & -1.387 & .842 \\
\hline
\end{tabular}


TOTAL VIIIb - Financial availability readiness

\begin{tabular}{|c|c|c|c|c|c|c|c|}
\hline \multirow{2}{*}{ IVs } & \multicolumn{2}{|c|}{$\begin{array}{l}\text { Unstandardised } \\
\text { Coefficients }\end{array}$} & \multirow{2}{*}{$\beta$} & \multirow{2}{*}{$\mathrm{t}$} & \multirow{2}{*}{$\begin{array}{c}\mathrm{p} \\
\text { value }\end{array}$} & \multicolumn{2}{|c|}{$\begin{array}{c}95 \% \text { Confidence } \\
\text { Interval for B }\end{array}$} \\
\hline & B & $\begin{array}{l}\text { Std. } \\
\text { Error }\end{array}$ & & & & $\begin{array}{l}\text { Lower } \\
\text { Bound }\end{array}$ & $\begin{array}{l}\text { Upper } \\
\text { Bound }\end{array}$ \\
\hline Education & .328 & .171 & .153 & 1.921 & .056 & -.008 & .664 \\
\hline Gender & -.266 & .197 & -.083 & -1.351 & .178 & -.653 & .122 \\
\hline Age & -.076 & .111 & -.047 & -.691 & .490 & -.294 & .141 \\
\hline B1atwork & -.191 & .266 & -.044 & -.716 & .475 & -.715 & .334 \\
\hline B2athome & .002 & .227 & .001 & .009 & .993 & -.445 & .449 \\
\hline Jobtitle1 & -.164 & .260 & -.044 & -.630 & .529 & -.675 & .348 \\
\hline Jobtitle2 & .052 & .247 & .017 & .213 & .832 & -.433 & .538 \\
\hline Jobtitle3 & .277 & .316 & .067 & .876 & .382 & -.345 & .898 \\
\hline
\end{tabular}

TOTAL VIIIc - Financial possibility readiness

\begin{tabular}{|c|c|c|c|c|c|c|c|}
\hline \multirow{2}{*}{ IVs } & \multicolumn{2}{|c|}{$\begin{array}{l}\text { Unstandardised } \\
\text { Coefficients }\end{array}$} & \multirow{2}{*}{ ß } & \multirow{2}{*}{$\mathrm{t}$} & \multirow{2}{*}{$\begin{array}{c}p \\
\text { value }\end{array}$} & \multicolumn{2}{|c|}{$\begin{array}{l}95 \% \text { Confidence } \\
\text { Interval for B }\end{array}$} \\
\hline & B & $\begin{array}{l}\text { Std. } \\
\text { Error }\end{array}$ & & & & $\begin{array}{l}\text { Lower } \\
\text { Bound }\end{array}$ & $\begin{array}{l}\text { Upper } \\
\text { Bound }\end{array}$ \\
\hline Gender & -.552 & .218 & -.157 & -2.533 & .012 & -.981 & -.123 \\
\hline B1atwork & -.373 & .295 & -.079 & -1.265 & .207 & -.954 & .208 \\
\hline Education & .177 & .189 & .075 & .934 & .351 & -196 & .549 \\
\hline Age & .119 & .123 & .067 & .972 & .332 & -.122 & .360 \\
\hline B2athome & .126 & .252 & .031 & .499 & .618 & -.370 & .621 \\
\hline Jobtitle1 & .082 & .288 & .020 & .286 & .775 & -.484 & .649 \\
\hline Jobtitle2 & -.085 & .273 & -.025 & -.313 & .755 & -.623 & .452 \\
\hline Jobtitle3 & -.136 & .350 & -.030 & -.389 & .697 & -.825 & .552 \\
\hline
\end{tabular}




\section{Appendix 8: Summary of survey results}

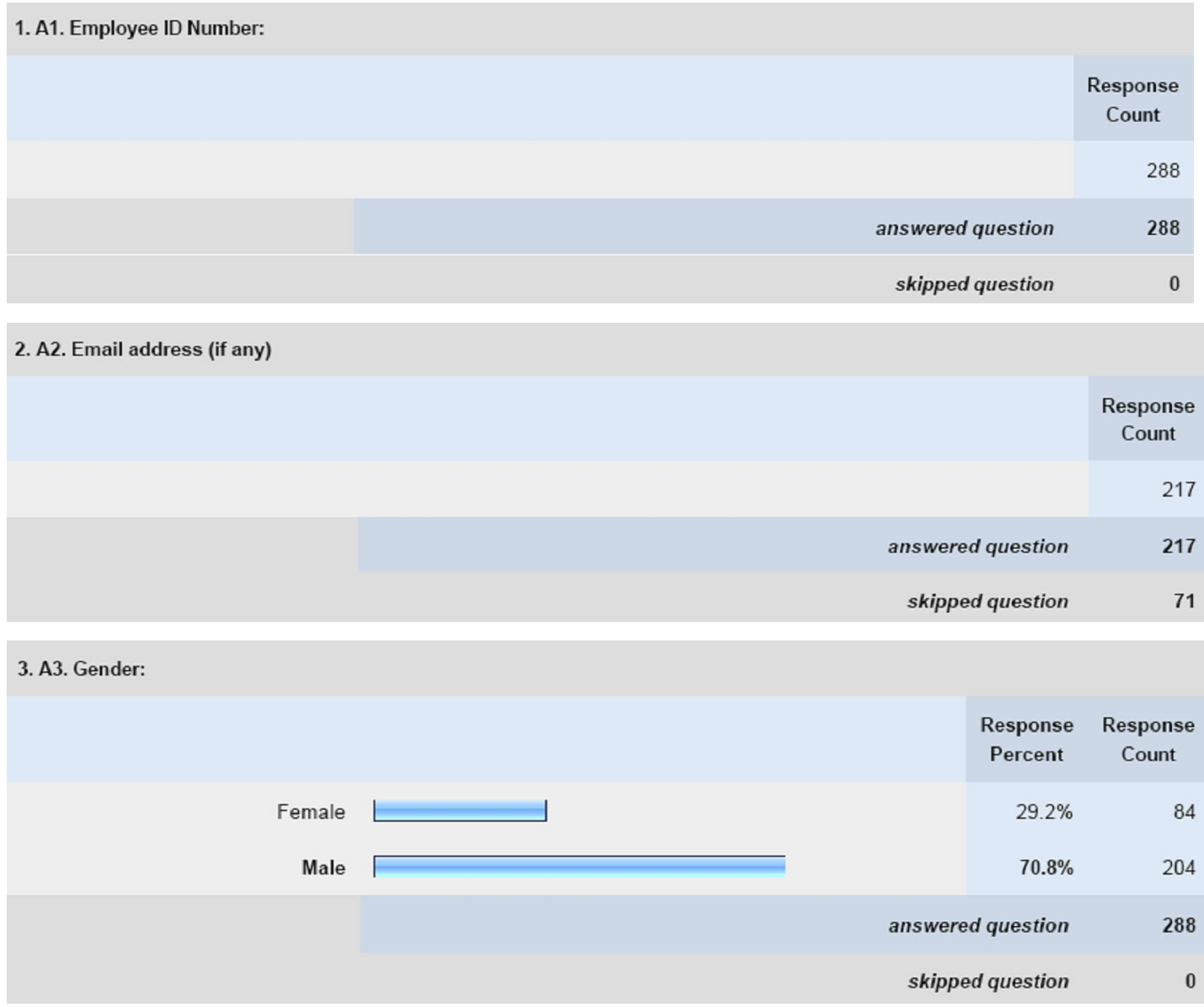

4. A4. Age:

Response
Rercent $\begin{gathered}\text { Count } \\ \text { Perponse }\end{gathered}$




\begin{tabular}{|c|c|c|c|}
\hline & & $\begin{array}{c}\text { Response } \\
\text { Percent }\end{array}$ & $\begin{array}{c}\text { Response } \\
\text { Count }\end{array}$ \\
\hline High school & & $22.6 \%$ & 65 \\
\hline Certificate/Diploma & П & $3.8 \%$ & 11 \\
\hline Bachelors degree & ${ }^{L}$ & $49.7 \%$ & 143 \\
\hline Postgraduate diploma & $\sqcap$ & $3.8 \%$ & 11 \\
\hline Master & $\rightleftarrows$ & $18.4 \%$ & 53 \\
\hline $\mathrm{PhD}$ & 月 & $1.4 \%$ & 4 \\
\hline other, please specify: & 『 & $0.3 \%$ & 1 \\
\hline \multicolumn{3}{|c|}{ answered question } & 288 \\
\hline \multicolumn{3}{|c|}{ skipped question } & $\mathbf{0}$ \\
\hline
\end{tabular}




\begin{tabular}{|c|c|c|c|}
\hline & & $\begin{array}{l}\text { Response } \\
\text { Percent }\end{array}$ & $\begin{array}{l}\text { Response } \\
\text { Count }\end{array}$ \\
\hline 1 Secretariat of FORDA, JAKARTA & $1-1$ & $9.4 \%$ & 27 \\
\hline $\begin{array}{r}2 \text { Centre for Forest and Nature } \\
\text { Conservation Research \& } \\
\text { Development, BOGOR }\end{array}$ & $\square$ & $5.6 \%$ & 16 \\
\hline $\begin{array}{l}3 \text { Centre for Forest Products } \\
\text { Research \& Development, BOGOR }\end{array}$ & & $0.0 \%$ & 0 \\
\hline $\begin{array}{l}4 \text { Centre for Socio-Economic and } \\
\text { Forestry Policy Research, BOGOR }\end{array}$ & 1 & $3.1 \%$ & 9 \\
\hline $\begin{array}{l}5 \text { Centre for Plantation Forest } \\
\text { Research and Development, } \\
\text { BOGOR }\end{array}$ & $\sqcap$ & $3.5 \%$ & 10 \\
\hline $\begin{array}{l}6 \text { Centre for Dipterocarp Research, } \\
\text { SAMARINDA }\end{array}$ & $\square$ & $4.2 \%$ & 12 \\
\hline $\begin{array}{l}7 \text { Centre for Biotechnology and } \\
\text { Forest Tree Improvement Research, } \\
\text { YOGYAKARTA }\end{array}$ & 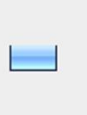 & $6.6 \%$ & 19 \\
\hline $\begin{array}{r}8 \text { Forestry Research Institute of AEK } \\
\text { NAULI }\end{array}$ & $1-1$ & $6.6 \%$ & 19 \\
\hline $\begin{array}{r}9 \text { Forestry Research Institute of } \\
\text { MAKASSAR }\end{array}$ & $\square$ & $9.4 \%$ & 27 \\
\hline $\begin{array}{r}10 \text { Forestry Research Institute of } \\
\text { KUPANG }\end{array}$ & $\square$ & $6.6 \%$ & 19 \\
\hline $\begin{array}{r}11 \text { Forestry Research Institute of } \\
\text { MANOKWARI }\end{array}$ & 1 & $5.2 \%$ & 15 \\
\hline $\begin{array}{r}12 \text { Forestry Research Institute of } \\
\text { PALEMBANG }\end{array}$ & $\square$ & $6.6 \%$ & 19 \\
\hline $\begin{array}{r}13 \text { Forestry Research Institute of } \\
\text { BANJARBARU }\end{array}$ & 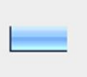 & $8.3 \%$ & 24 \\
\hline $\begin{array}{r}14 \text { Forestry Research Institute of } \\
\text { MATARAM }\end{array}$ & $\sqcap$ & $4.5 \%$ & 13 \\
\hline $\begin{array}{r}15 \text { Forestry Research Institute of } \\
\text { MANADO }\end{array}$ & E & $2.1 \%$ & 6 \\
\hline $\begin{array}{r}16 \text { Forestry Research Institute of } \\
\text { SURAKARTA }\end{array}$ & $L$ & $4.9 \%$ & 14 \\
\hline $\begin{array}{r}17 \text { Forestry Research Institute of } \\
\text { CIAMIS }\end{array}$ & $\square$ & $3.8 \%$ & 11 \\
\hline $\begin{array}{l}18 \text { Forest Breeding Technology } \\
\text { Research Institute of BOGOR }\end{array}$ & U & $1.4 \%$ & 4 \\
\hline $\begin{array}{l}19 \text { Forest Breeding Technology } \\
\text { Research Institute of SAMBOJA }\end{array}$ & $\Gamma$ & $3.1 \%$ & 9 \\
\hline $\begin{array}{r}20 \text { Pulp and Fiber Forest Products } \\
\text { Research Institute of KUOK }\end{array}$ & $\square$ & $5.2 \%$ & 15 \\
\hline & & answered question & 288 \\
\hline & & skipped question & 0 \\
\hline
\end{tabular}




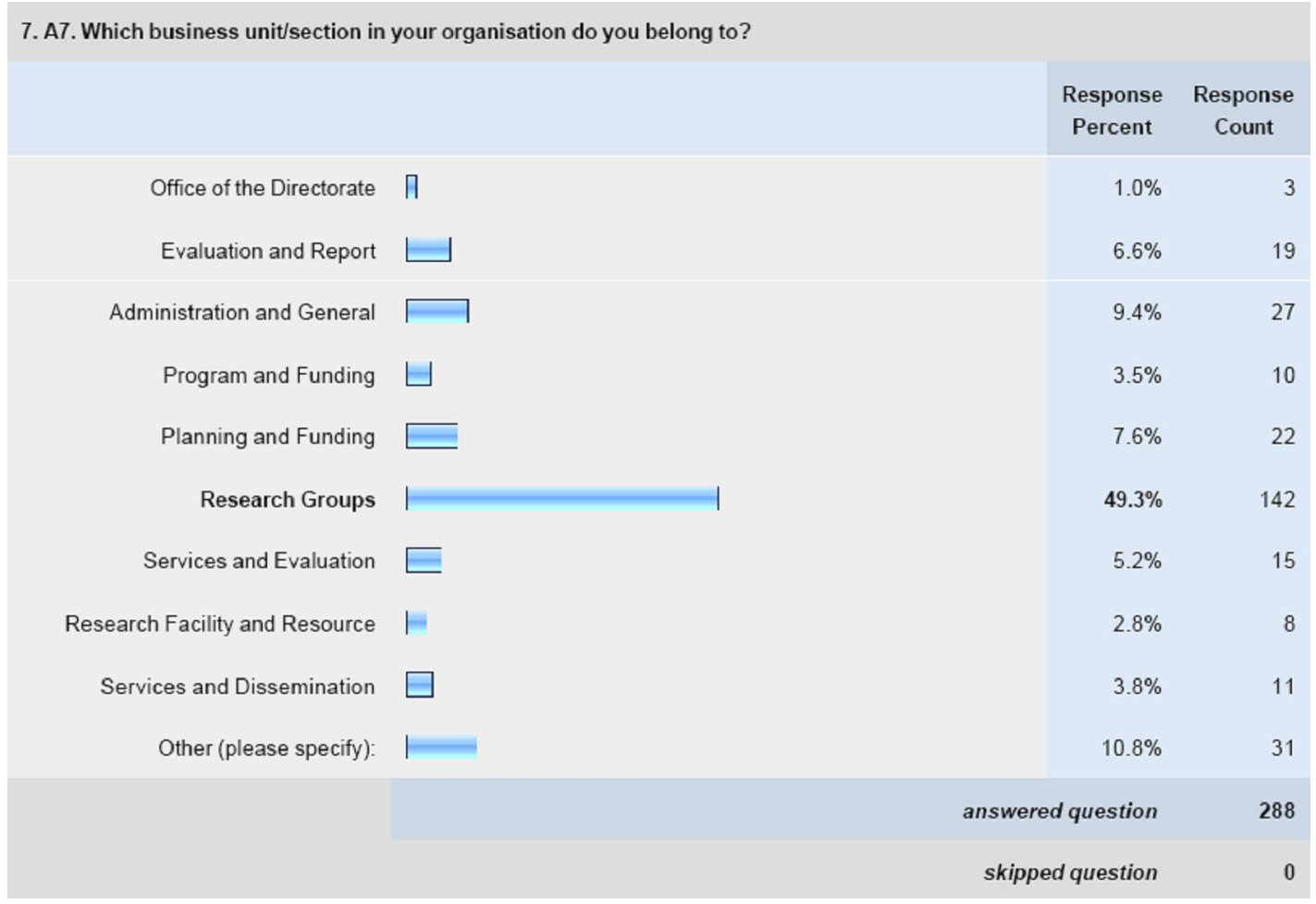

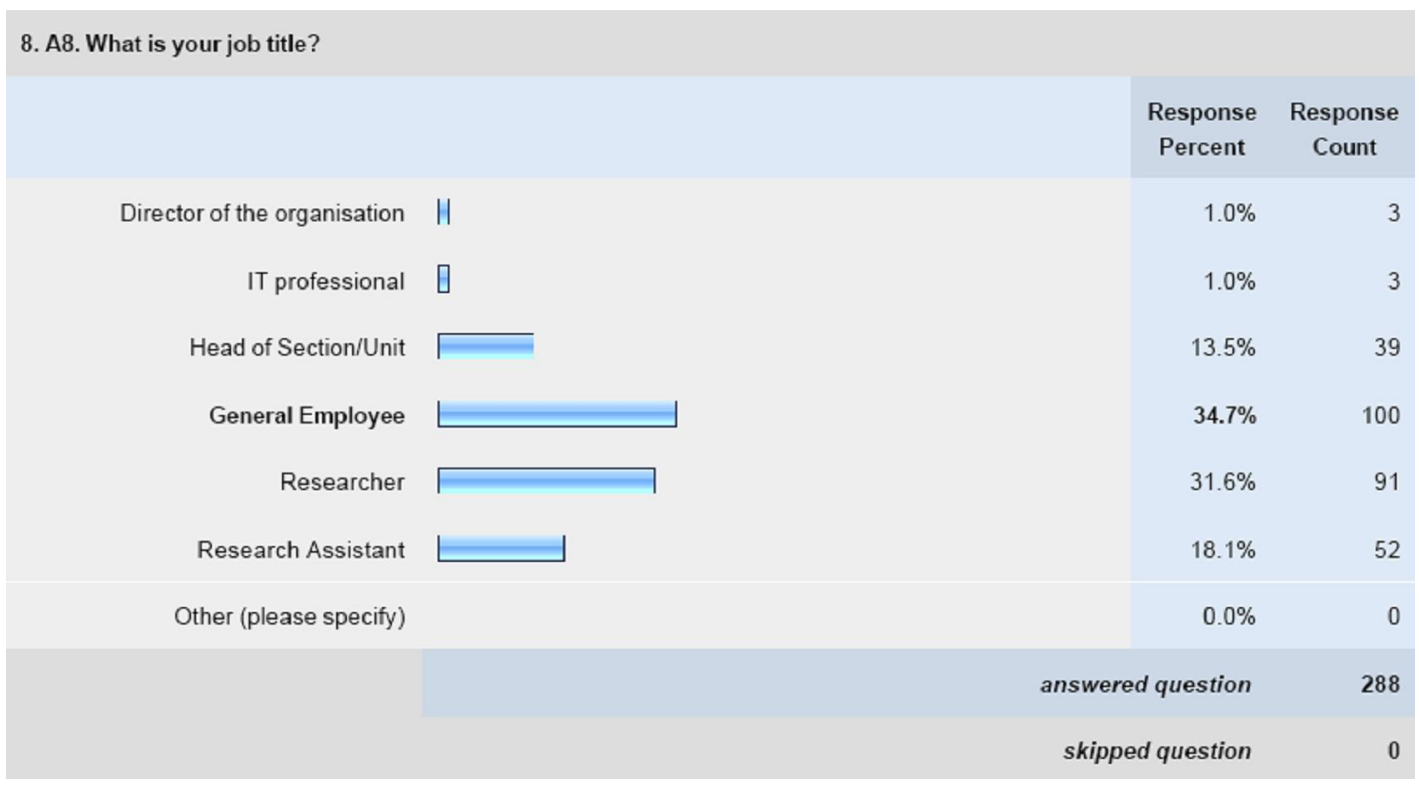

9. A9. Please provide a brief description of your job?

Response
Count
0
0
0
answered question
skipped question




\begin{tabular}{|c|c|c|c|}
\hline & & $\begin{array}{c}\text { Response } \\
\text { Percent }\end{array}$ & $\begin{array}{c}\text { Response } \\
\text { Count }\end{array}$ \\
\hline Yes & $L$ & $86.8 \%$ & 250 \\
\hline No & $\square$ & $13.2 \%$ & 38 \\
\hline \multicolumn{3}{|c|}{ answered question } & 288 \\
\hline \multicolumn{3}{|c|}{ skipped question } & 0 \\
\hline
\end{tabular}

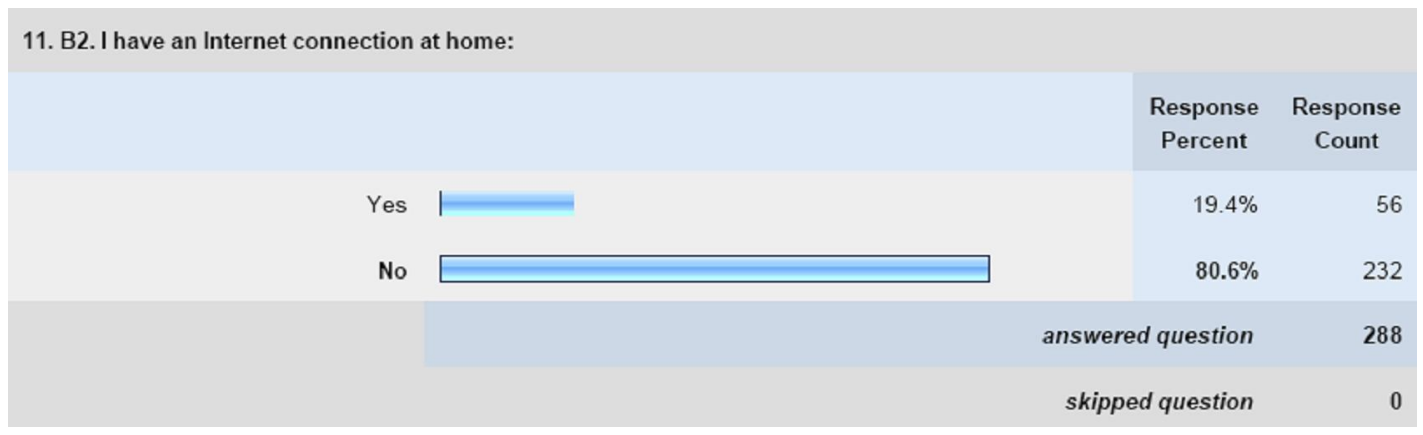

12. B3. I access the Internet mostly from:

\begin{tabular}{|c|c|c|c|}
\hline & & $\begin{array}{c}\text { Response } \\
\text { Percent }\end{array}$ & $\begin{array}{c}\text { Response } \\
\text { Count }\end{array}$ \\
\hline Home & E & $1.7 \%$ & 5 \\
\hline Workplace & 1 & $78.5 \%$ & 226 \\
\hline Cyber/Internet café & $\square$ & $10.8 \%$ & 31 \\
\hline College/university & & $0.0 \%$ & 0 \\
\hline Wi-Fi Hot Spots & E & $2.1 \%$ & 6 \\
\hline Mobile phone/PDA & 』 & $0.3 \%$ & 1 \\
\hline None & $\square$ & $6.6 \%$ & 19 \\
\hline Other please specify & & $0.0 \%$ & 0 \\
\hline \multicolumn{3}{|c|}{ answered question } & 288 \\
\hline \multicolumn{3}{|c|}{ skipped question } & 0 \\
\hline
\end{tabular}

13. B4. Are you a member of any social networking sites?

\begin{tabular}{|c|c|c|c|}
\hline & & $\begin{array}{c}\text { Response } \\
\text { Percent }\end{array}$ & $\begin{array}{c}\text { Response } \\
\text { Count }\end{array}$ \\
\hline No (skip to question B6) & 1 & $66.3 \%$ & 191 \\
\hline Yes, How many: & $\bar{\square}$ & $33.7 \%$ & 97 \\
\hline \multicolumn{3}{|c|}{ answered question } & 288 \\
\hline \multicolumn{3}{|c|}{ skipped question } & 0 \\
\hline
\end{tabular}




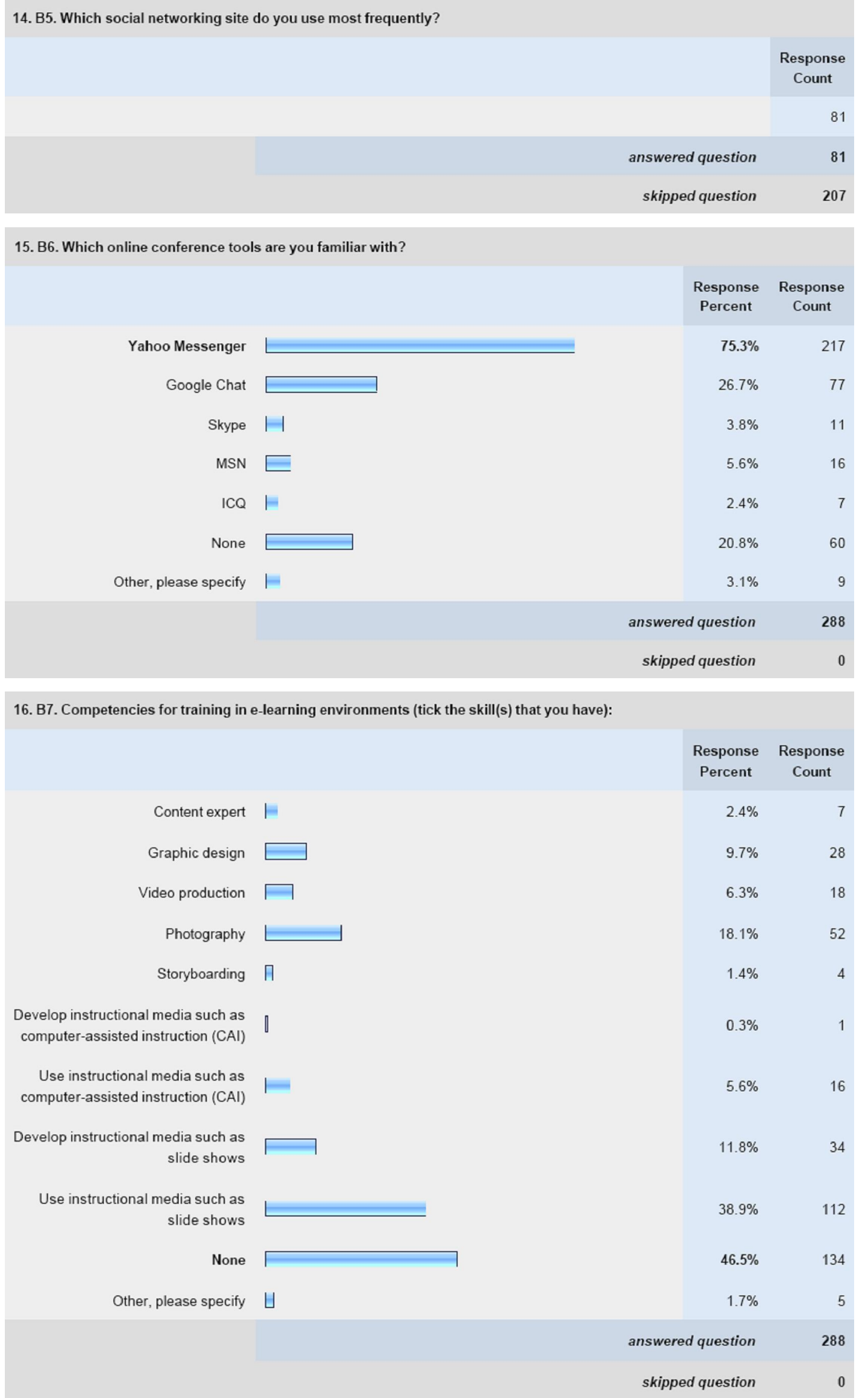




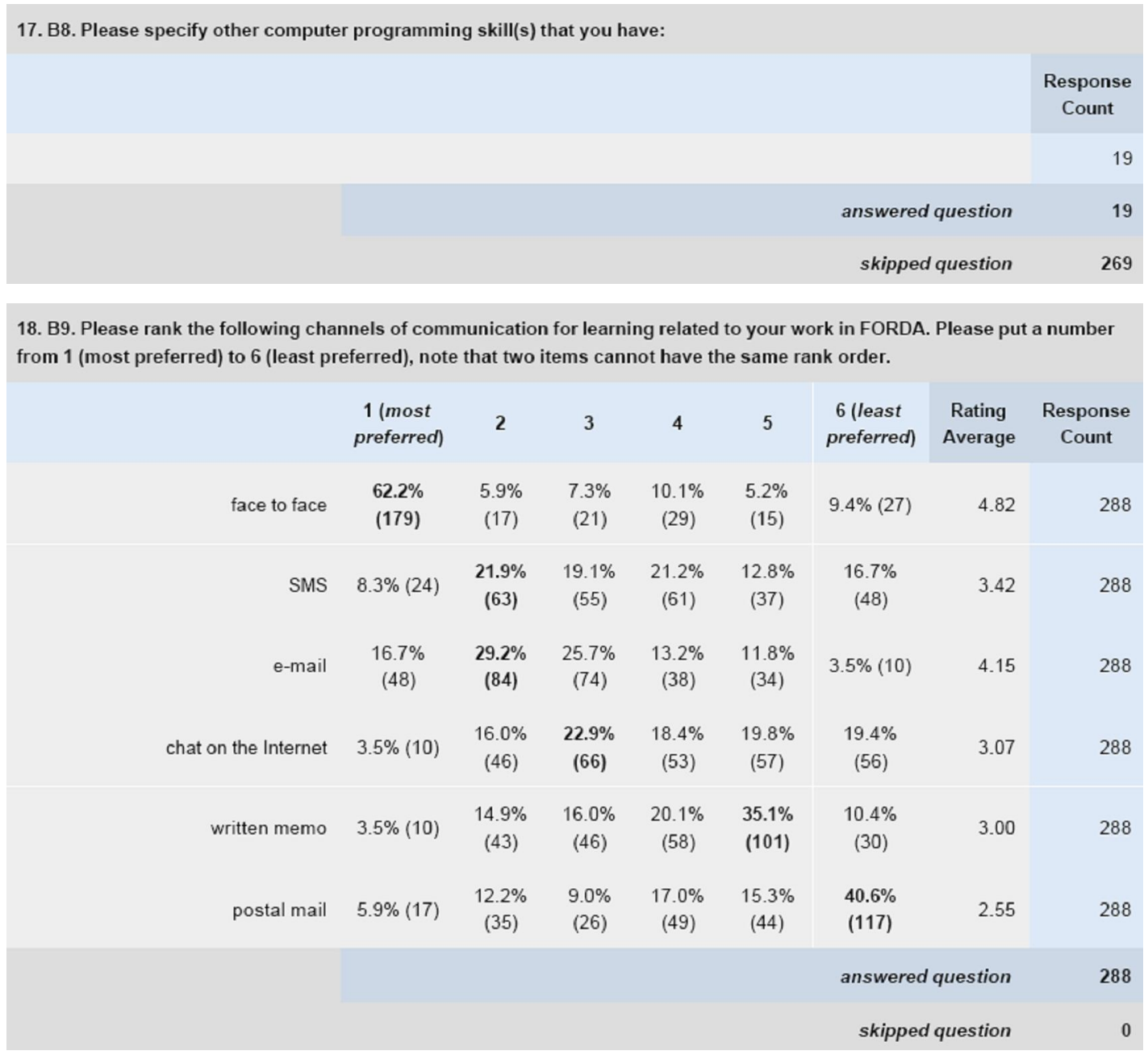




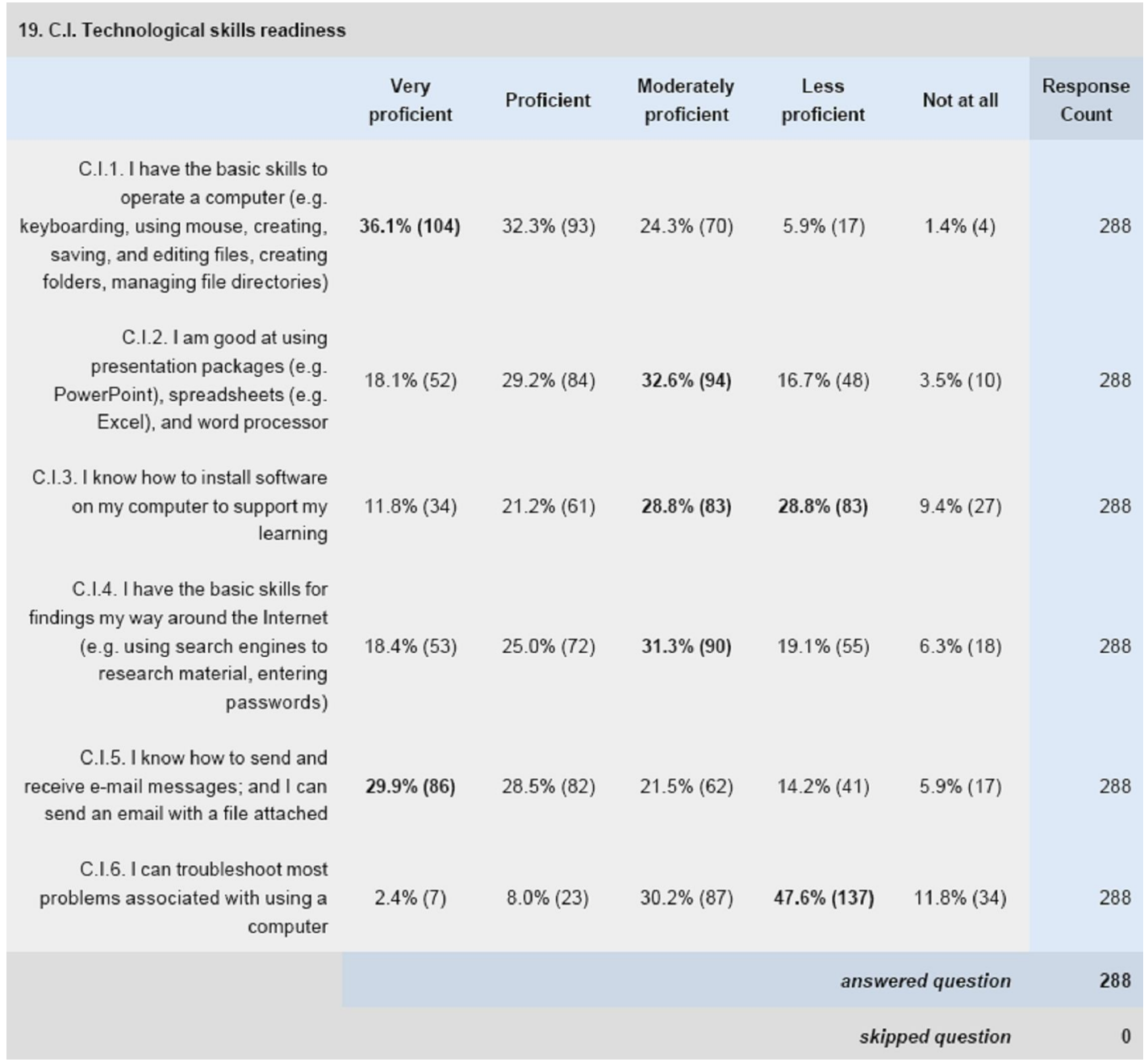




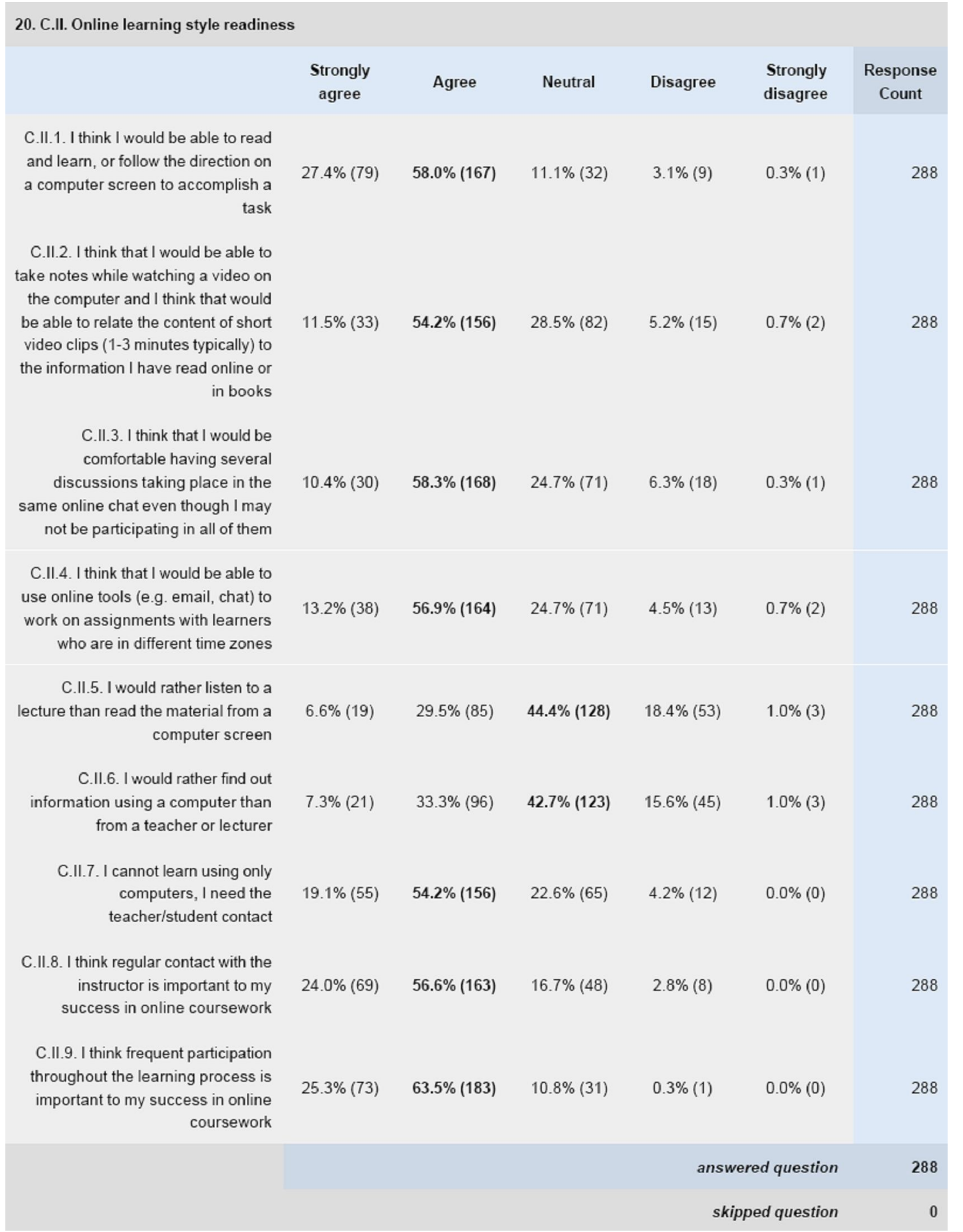




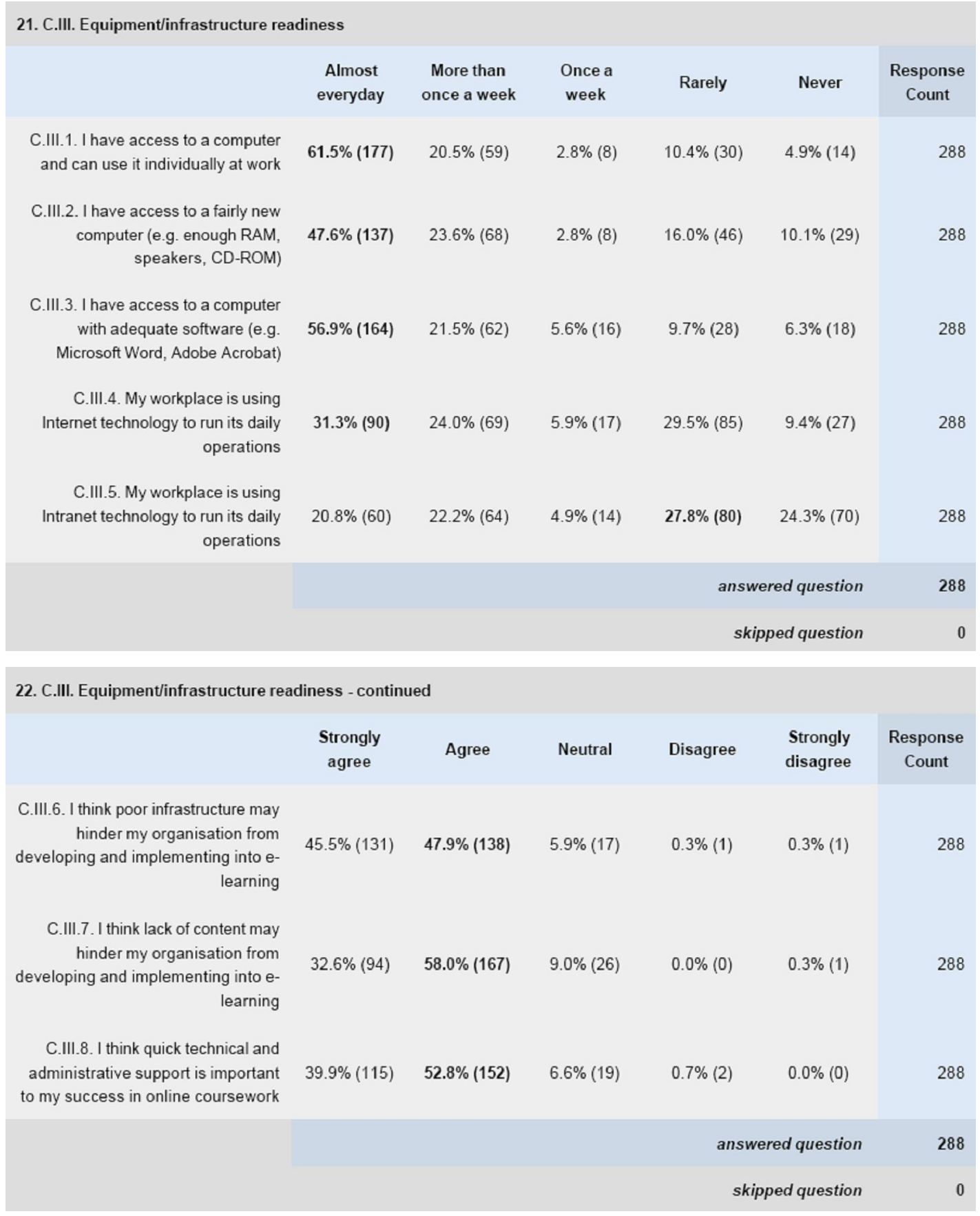




\begin{tabular}{|c|c|c|c|c|c|c|}
\hline \multicolumn{7}{|l|}{ 23. Confidence } \\
\hline & $\begin{array}{l}\text { Strongly } \\
\text { agree }\end{array}$ & Agree & Neutral & Disagree & $\begin{array}{l}\text { Strongly } \\
\text { disagree }\end{array}$ & $\begin{array}{l}\text { Response } \\
\text { Count }\end{array}$ \\
\hline $\begin{array}{r}\text { C.IV.1. I feel confidence in my ability } \\
\text { to use advanced technology in } \\
\text { training and/or learning }\end{array}$ & $22.6 \%(65)$ & $57.6 \%(166)$ & $18.1 \%(52)$ & $1.7 \%(5)$ & $0.0 \%(0)$ & 288 \\
\hline $\begin{array}{l}\text { C.IV.2. I hesitate to use technology } \\
\text { for fear of making mistakes }\end{array}$ & $0.0 \%(0)$ & $4.2 \%(12)$ & $9.4 \%(27)$ & $63.2 \%(182)$ & $23.3 \%(67)$ & 288 \\
\hline $\begin{array}{l}\text { C.IV.3. I can teach myself most of the } \\
\text { things I need to know about using e- } \\
\text { learning technologies }\end{array}$ & $5.2 \%(15)$ & $23.3 \%(67)$ & $40.3 \%(116)$ & $29.2 \%(84)$ & $2.1 \%(6)$ & 288 \\
\hline $\begin{array}{r}\text { C.IV.4. I would feel better about } \\
\text { using technology if I knew more } \\
\text { about them }\end{array}$ & $35.8 \%(103)$ & $55.2 \%(159)$ & $8.0 \%(23)$ & $1.0 \%(3)$ & $0.0 \%(0)$ & 288 \\
\hline $\begin{array}{r}\text { C.IV.5. I feel threatened when I see } \\
\text { others using technology in their } \\
\text { learning/working }\end{array}$ & $1.4 \%(4)$ & $5.6 \%(16)$ & $19.1 \%(55)$ & $50.7 \%(146)$ & $23.3 \%(67)$ & 288 \\
\hline $\begin{array}{r}\text { C.IV.6. Knowing how to use e- } \\
\text { learning technologies will increase } \\
\text { my job possibilities }\end{array}$ & $33.0 \%(95)$ & $58.0 \%(167)$ & $8.7 \%(25)$ & $0.3 \%(1)$ & $0.0 \%(0)$ & 288 \\
\hline & & & & \multicolumn{2}{|c|}{ answered question } & 288 \\
\hline & & & & \multicolumn{2}{|c|}{ skipped question } & 0 \\
\hline \multicolumn{7}{|l|}{ 24. Enjoyment } \\
\hline & $\begin{array}{l}\text { Strongly } \\
\text { agree }\end{array}$ & Agree & Neutral & Disagree & $\begin{array}{l}\text { Strongly } \\
\text { disagree }\end{array}$ & $\begin{array}{l}\text { Response } \\
\text { Count }\end{array}$ \\
\hline $\begin{array}{r}\text { C.IV.7. I like using computers for } \\
\text { research }\end{array}$ & $26.4 \%(76)$ & $51.7 \%(149)$ & $18.8 \%(54)$ & $2.4 \%(7)$ & $0.7 \%(2)$ & 288 \\
\hline $\begin{array}{r}\text { C.IV.8. I like to communicate with } \\
\text { others using e-mail to support my } \\
\text { learning }\end{array}$ & $22.6 \%(65)$ & $53.1 \%(153)$ & $21.9 \%(63)$ & $2.1 \%(6)$ & $0.3 \%(1)$ & 288 \\
\hline $\begin{array}{r}\text { C.IV.9. I spend a lot of time on the } \\
\text { Internet }\end{array}$ & $5.6 \%(16)$ & $19.4 \%(56)$ & $45.1 \%(130)$ & $26.7 \%(77)$ & $3.1 \%(9)$ & 288 \\
\hline $\begin{array}{r}\text { C.IV.10. I enjoy working on tasks on } \\
\text { a computer that I can do by following } \\
\text { directions }\end{array}$ & $15.3 \%(44)$ & $62.8 \%(181)$ & $19.1 \%(55)$ & $2.4 \%(7)$ & $0.3 \%(1)$ & 288 \\
\hline \multirow[t]{3}{*}{$\begin{array}{l}\text { C.IV.11. I like to try new technologies } \\
\text { for teaching, training and/or learning }\end{array}$} & $18.8 \%(54)$ & $56.6 \%(163)$ & $24.0 \%(69)$ & $0.3 \%(1)$ & $0.3 \%(1)$ & 288 \\
\hline & & & & \multicolumn{2}{|c|}{ answered question } & 288 \\
\hline & & & & \multicolumn{2}{|c|}{ skipped question } & $\mathbf{0}$ \\
\hline
\end{tabular}




\begin{tabular}{|c|c|c|c|c|c|c|}
\hline \multicolumn{7}{|l|}{ 25. Importance } \\
\hline & $\begin{array}{c}\text { Strongly } \\
\text { agree }\end{array}$ & Agree & Neutral & Disagree & $\begin{array}{l}\text { Strongly } \\
\text { disagree }\end{array}$ & $\begin{array}{c}\text { Response } \\
\text { Count }\end{array}$ \\
\hline $\begin{array}{l}\text { C.IV.12. It is important to learn how } \\
\text { to use a computer for learning and } \\
\text { training, and how to use the Internet } \\
\text { as a learning resource }\end{array}$ & $47.6 \%(137)$ & $49.0 \%(141)$ & $3.5 \%(10)$ & $0.0 \%(0)$ & $0.0 \%(0)$ & 288 \\
\hline \multirow[t]{3}{*}{$\begin{array}{r}\text { C.IV.13. Learning about how to use } \\
\text { e-learning technologies is a waste } \\
\text { of time }\end{array}$} & $0.0 \%(0)$ & $0.7 \%(2)$ & $6.3 \%(18)$ & $60.8 \%(175)$ & $32.3 \%(93)$ & 288 \\
\hline & & & & \multicolumn{2}{|c|}{ answered question } & 288 \\
\hline & & & & \multicolumn{2}{|c|}{ skipped question } & 0 \\
\hline
\end{tabular}

\begin{tabular}{|c|c|c|c|c|c|c|}
\hline \multicolumn{7}{|l|}{ 26. Motivation } \\
\hline & $\begin{array}{l}\text { Strongly } \\
\text { agree }\end{array}$ & Agree & Neutral & Disagree & $\begin{array}{l}\text { Strongly } \\
\text { disagree }\end{array}$ & $\begin{array}{c}\text { Response } \\
\text { Count }\end{array}$ \\
\hline $\begin{array}{l}\text { C.IV.14. I think that I would be able to } \\
\text { remain motivated even though the } \\
\text { instructor is not online at all times }\end{array}$ & $6.9 \%(20)$ & $54.5 \%(157)$ & $32.6 \%(94)$ & $5.2 \%(15)$ & $0.7 \%(2)$ & 288 \\
\hline $\begin{array}{l}\text { C.IV.15. I think that I would be able to } \\
\text { complete my work even when there } \\
\text { are online distractions (e.g. friends } \\
\text { sending emails or Websites to surf) }\end{array}$ & $4.9 \%(14)$ & $47.9 \%(138)$ & $30.6 \%(88)$ & $15.3 \%(44)$ & $1.4 \%(4)$ & 288 \\
\hline \multirow[t]{3}{*}{$\begin{array}{l}\text { C.IV.16. I think that I would be able to } \\
\text { complete my work even when there } \\
\text { are distractions in my home (e.g. } \\
\text { television, children, and such) }\end{array}$} & $4.5 \%(13)$ & $41.0 \%(118)$ & $36.1 \%(104)$ & $17.0 \%(49)$ & $1.4 \%(4)$ & 288 \\
\hline & & & & \multicolumn{2}{|c|}{ answered question } & 288 \\
\hline & & & & \multicolumn{2}{|c|}{ skipped question } & 0 \\
\hline
\end{tabular}

\begin{tabular}{|c|c|c|c|c|c|c|}
\hline \multicolumn{7}{|l|}{ 27. Self-development } \\
\hline & $\begin{array}{c}\text { Strongly } \\
\text { agree }\end{array}$ & Agree & Neutral & Disagree & $\begin{array}{l}\text { Strongly } \\
\text { disagree }\end{array}$ & $\begin{array}{c}\text { Response } \\
\text { Count }\end{array}$ \\
\hline $\begin{array}{r}\text { C.IV.17. I think I can spend some } \\
\text { time ( } 15,30 \text {, or } 60 \text { minutes) for } \\
\text { improving myself during some part } \\
\text { of the day (morning, afternoon, } \\
\text { evening, or night) }\end{array}$ & $22.2 \%(64)$ & $62.5 \%(180)$ & $13.9 \%(40)$ & $1.4 \%(4)$ & $0.0 \%(0)$ & 288 \\
\hline $\begin{array}{l}\text { C.IV.18. I believe that self- } \\
\text { development of employees may } \\
\text { strengthen the position of the unit in } \\
\text { the organisation }\end{array}$ & $37.8 \%(109)$ & $54.9 \%(158)$ & $7.3 \%(21)$ & $0.0 \%(0)$ & $0.0 \%(0)$ & 288 \\
\hline \multirow[t]{3}{*}{$\begin{array}{r}\text { C.IV.19. I am interested to upgrade } \\
\text { my academic/professional } \\
\text { qualification and/or work } \\
\text { performance through e-learning }\end{array}$} & $34.0 \%(98)$ & $54.9 \%(158)$ & $10.4 \%(30)$ & $0.7 \%(2)$ & $0.0 \%(0)$ & 288 \\
\hline & & & & \multicolumn{2}{|c|}{ answered question } & 288 \\
\hline & & & & \multicolumn{2}{|c|}{ skipped question } & 0 \\
\hline
\end{tabular}




\begin{tabular}{|c|c|c|c|c|c|c|}
\hline \multicolumn{7}{|l|}{ 28. Anxiety } \\
\hline & $\begin{array}{l}\text { Strongly } \\
\text { agree }\end{array}$ & Agree & Neutral & Disagree & $\begin{array}{l}\text { Strongly } \\
\text { disagree }\end{array}$ & $\begin{array}{l}\text { Response } \\
\text { Count }\end{array}$ \\
\hline $\begin{array}{r}\text { C.IV.20. I think positively toward the } \\
\text { technological interventions in } \\
\text { daily/routine tasks }\end{array}$ & $25.0 \%(72)$ & $63.5 \%(183)$ & $11.1 \%(32)$ & $0.3 \%(1)$ & $0.0 \%(0)$ & 288 \\
\hline $\begin{array}{r}\text { C.IV.21. I feel comfortable with the } \\
\text { thought of using technology to } \\
\text { deliver instruction }\end{array}$ & $18.4 \%(53)$ & $63.9 \%(184)$ & $14.9 \%(43)$ & $2.8 \%(8)$ & $0.0 \%(0)$ & 288 \\
\hline $\begin{array}{r}\text { C.IV.22. The thought of using e- } \\
\text { learning technologies makes me } \\
\text { very nervous }\end{array}$ & $0.0 \%(0)$ & $2.8 \%(8)$ & $28.1 \%(81)$ & $56.3 \%(162)$ & $12.8 \%(37)$ & 288 \\
\hline $\begin{array}{l}\text { C.IV.23. I get a sinking feeling when I } \\
\text { think of trying to use technology for } \\
\text { training trainees and/or learning }\end{array}$ & $0.7 \%(2)$ & $4.9 \%(14)$ & $21.5 \%(62)$ & $57.3 \%(165)$ & $15.6 \%(45)$ & 288 \\
\hline & & & & \multicolumn{2}{|c|}{ answered question } & 288 \\
\hline & & & & \multicolumn{2}{|c|}{ skipped question } & 0 \\
\hline \multicolumn{7}{|l|}{ 29. Management } \\
\hline & $\begin{array}{l}\text { Strongly } \\
\text { agree }\end{array}$ & Agree & Neutral & Disagree & $\begin{array}{l}\text { Strongly } \\
\text { disagree }\end{array}$ & $\begin{array}{l}\text { Response } \\
\text { Count }\end{array}$ \\
\hline $\begin{array}{l}\text { C.V.1. My workplace has a } \\
\text { vision/mission on e-learning }\end{array}$ & $12.8 \%(37)$ & $44.1 \%(127)$ & $36.8 \%(106)$ & $5.6 \%(16)$ & $0.7 \%(2)$ & 288 \\
\hline $\begin{array}{r}\text { C.V.2. The high and mid level } \\
\text { managers think positively toward the } \\
\text { technological interventions in } \\
\text { daily/routine tasks }\end{array}$ & $10.1 \%(29)$ & $56.3 \%(162)$ & $29.9 \%(86)$ & $3.8 \%(11)$ & $0.0 \%(0)$ & 288 \\
\hline $\begin{array}{l}\text { C.V.3. The majority of high and mid } \\
\text { level managers have accepted any } \\
\text { changes that required the use of } \\
\text { technology in daily/routine tasks }\end{array}$ & $9.4 \%(27)$ & $54.5 \%(157)$ & $30.9 \%(89)$ & $4.9 \%(14)$ & $0.3 \%(1)$ & 288 \\
\hline \multirow[t]{3}{*}{$\begin{array}{l}\text { C.V.4. My employer will give me the } \\
\text { time off to study via e-learning and } \\
\text { will let me use the facilities at work } \\
\text { outside office hours for e-learning }\end{array}$} & $14.6 \%(42)$ & $48.3 \%(139)$ & $31.6 \%(91)$ & $3.8 \%(11)$ & $1.7 \%(5)$ & 288 \\
\hline & & & & \multicolumn{2}{|c|}{ answered question } & 288 \\
\hline & & & & \multicolumn{2}{|c|}{ skipped question } & 0 \\
\hline
\end{tabular}




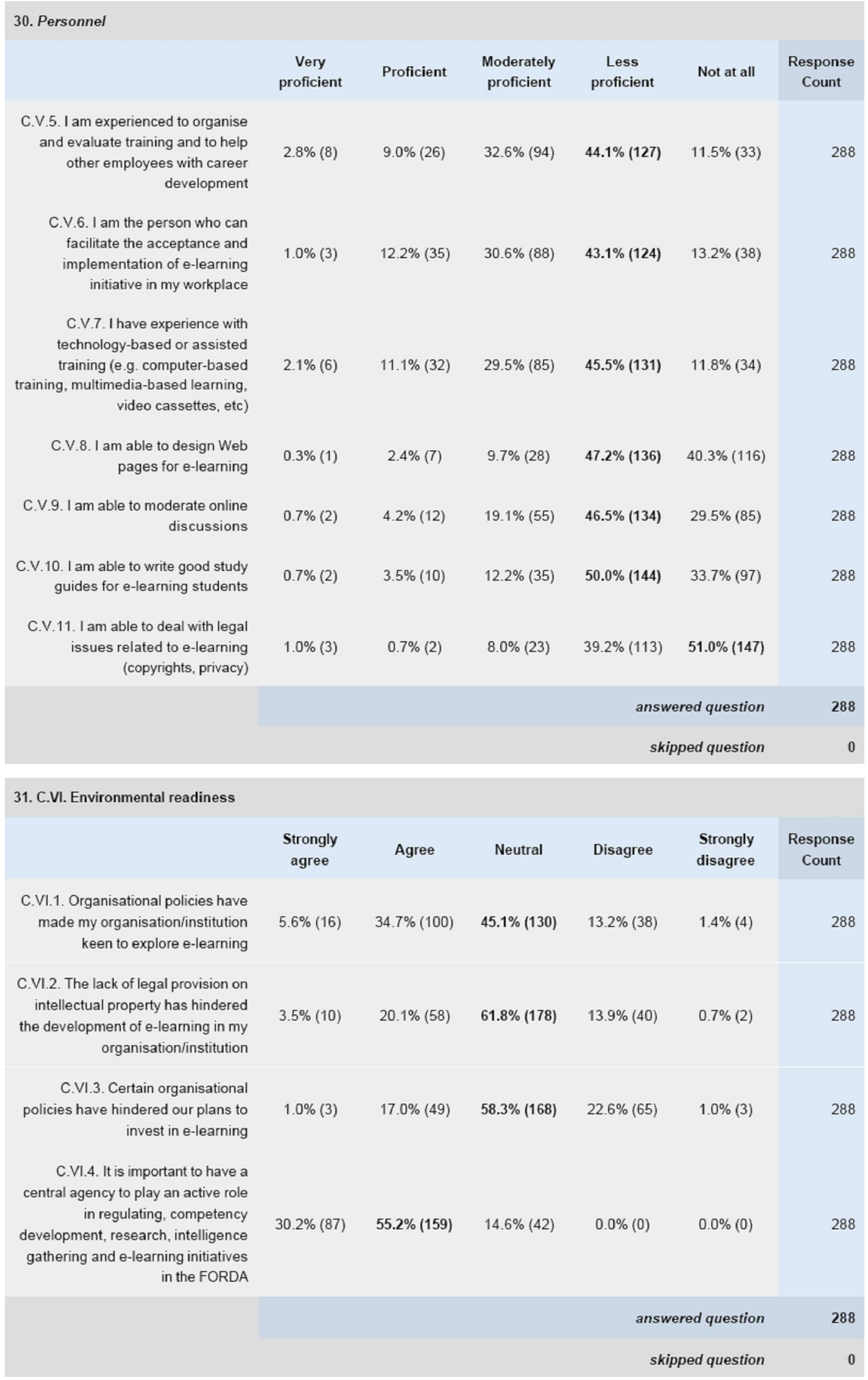




\begin{tabular}{|c|c|c|c|c|c|c|}
\hline \multicolumn{7}{|l|}{ 32. C.VII. Cultural readiness } \\
\hline & $\begin{array}{l}\text { Strongly } \\
\text { agree }\end{array}$ & Agree & Neutral & Disagree & $\begin{array}{l}\text { Strongly } \\
\text { disagree }\end{array}$ & $\begin{array}{l}\text { Response } \\
\text { Count }\end{array}$ \\
\hline $\begin{array}{r}\text { C.VII.1. I accepted technological } \\
\text { innovation (e.g. start using digital } \\
\text { documents instead of hard copies) } \\
\text { in routine/daily tasks }\end{array}$ & $18.4 \%(53)$ & $56.3 \%(162)$ & $23.3 \%(67)$ & $1.7 \%(5)$ & $0.3 \%(1)$ & 288 \\
\hline $\begin{array}{l}\text { C.VII.2. I have accepted any changes } \\
\text { that required the use of technology } \\
\text { in daily/routine tasks }\end{array}$ & $16.3 \%(47)$ & $61.5 \%(177)$ & $20.5 \%(59)$ & $1.4 \%(4)$ & $0.3 \%(1)$ & 288 \\
\hline $\begin{array}{l}\text { C.VII.3. The most effective method of } \\
\text { learning is face-to-face }\end{array}$ & $16.0 \%(46)$ & $37.5 \%(108)$ & $35.1 \%(101)$ & $10.8 \%(31)$ & $0.7 \%(2)$ & 288 \\
\hline $\begin{array}{l}\text { C.VII. } 4 \text {. The teacher/trainer/instructor } \\
\text { is still the best information provider }\end{array}$ & $14.9 \%(43)$ & $50.0 \%(144)$ & $26.0 \%(75)$ & $9.0 \%(26)$ & $0.0 \%(0)$ & 288 \\
\hline $\begin{array}{l}\text { C.VII.5. E-learning is an efficient } \\
\text { means of disseminating information }\end{array}$ & $21.2 \%(61)$ & $66.0 \%(190)$ & $12.8 \%(37)$ & $0.0 \%(0)$ & $0.0 \%(0)$ & 288 \\
\hline \multirow[t]{3}{*}{$\begin{array}{l}\text { C.VII.6. E-learning enables learners } \\
\text { and instructor to communicate and } \\
\text { interact better with one another }\end{array}$} & $11.8 \%(34)$ & $59.7 \%(172)$ & $25.0 \%(72)$ & $3.5 \%(10)$ & $0.0 \%(0)$ & 288 \\
\hline & & & & \multicolumn{2}{|c|}{ answered question } & 288 \\
\hline & & & & & questio & 0 \\
\hline
\end{tabular}

\begin{tabular}{|c|c|c|c|c|c|c|}
\hline \multicolumn{7}{|l|}{ 33. C.VIII. Financial readiness } \\
\hline & $\begin{array}{c}\text { Very } \\
\text { feasible }\end{array}$ & Feasible & $\begin{array}{c}\text { Somewhat } \\
\text { feasible }\end{array}$ & $\begin{array}{l}\text { Less } \\
\text { feasible }\end{array}$ & Not at all & $\begin{array}{l}\text { Response } \\
\text { Count }\end{array}$ \\
\hline $\begin{array}{r}\text { C.VIII.1. My organisation/institution } \\
\text { provides a computer loan to the } \\
\text { employees }\end{array}$ & $1.7 \%(5)$ & $10.1 \%(29)$ & $16.0 \%(46)$ & $16.3 \%(47)$ & $55.9 \%(161)$ & 288 \\
\hline $\begin{array}{l}\text { C.VIII.2. I can take a loan to buy a } \\
\text { computer for e-learning purposes }\end{array}$ & $4.2 \%(12)$ & $20.5 \%(59)$ & $33.0 \%(95)$ & $22.9 \%(66)$ & $19.4 \%(56)$ & 288 \\
\hline $\begin{array}{r}\text { C.VIII.3. I can afford to buy a } \\
\text { computer and pay for Internet } \\
\text { access }\end{array}$ & $5.9 \%(17)$ & $17.4 \%(50)$ & $32.6 \%(94)$ & $27.8 \%(80)$ & $16.3 \%(47)$ & 288 \\
\hline \multirow[t]{3}{*}{$\begin{array}{l}\text { C.VIII.4. My organisation/institution } \\
\text { provides funds for employees to } \\
\text { attend conferences and training }\end{array}$} & $4.9 \%(14)$ & $26.7 \%(77)$ & $35.1 \%(101)$ & $22.6 \%(65)$ & $10.8 \%(31)$ & 288 \\
\hline & & & & \multicolumn{2}{|c|}{ answered question } & 288 \\
\hline & & & & \multicolumn{2}{|c|}{ skipped question } & 0 \\
\hline
\end{tabular}




\begin{tabular}{|c|c|c|c|c|c|c|}
\hline & $\begin{array}{c}\text { Strongly } \\
\text { agree }\end{array}$ & Agree & Neutral & Disagree & $\begin{array}{l}\text { Strongly } \\
\text { disagree }\end{array}$ & $\begin{array}{c}\text { Response } \\
\text { Count }\end{array}$ \\
\hline $\begin{array}{l}\text { C.VIII.5. I think, my } \\
\text { organisation/institution can create a } \\
\text { budget for implementing e-learning }\end{array}$ & $20.1 \%(58)$ & $49.0 \%(141)$ & $28.1 \%(81)$ & $2.1 \%(6)$ & $0.7 \%(2)$ & 288 \\
\hline $\begin{array}{l}\text { C.VIII.6. I think high initial investment } \\
\text { may hinder my organisation from } \\
\text { developing and implementing into e- } \\
\text { learning }\end{array}$ & $4.9 \%(14)$ & $35.8 \%(103)$ & $37.8 \%(109)$ & $19.1 \%(55)$ & $2.4 \%(7)$ & 288 \\
\hline $\begin{array}{l}\text { C.VIII.7. I think high operating costs } \\
\text { may hinder my organisation from } \\
\text { developing and implementing into e- } \\
\text { learning }\end{array}$ & $5.6 \%(16)$ & $44.1 \%(127)$ & $35.1 \%(101)$ & $12.8 \%(37)$ & $2.4 \%(7)$ & 288 \\
\hline & & & & \multicolumn{2}{|c|}{ answered question } & 288 \\
\hline & & & & \multicolumn{2}{|c|}{ skipped question } & 0 \\
\hline
\end{tabular}

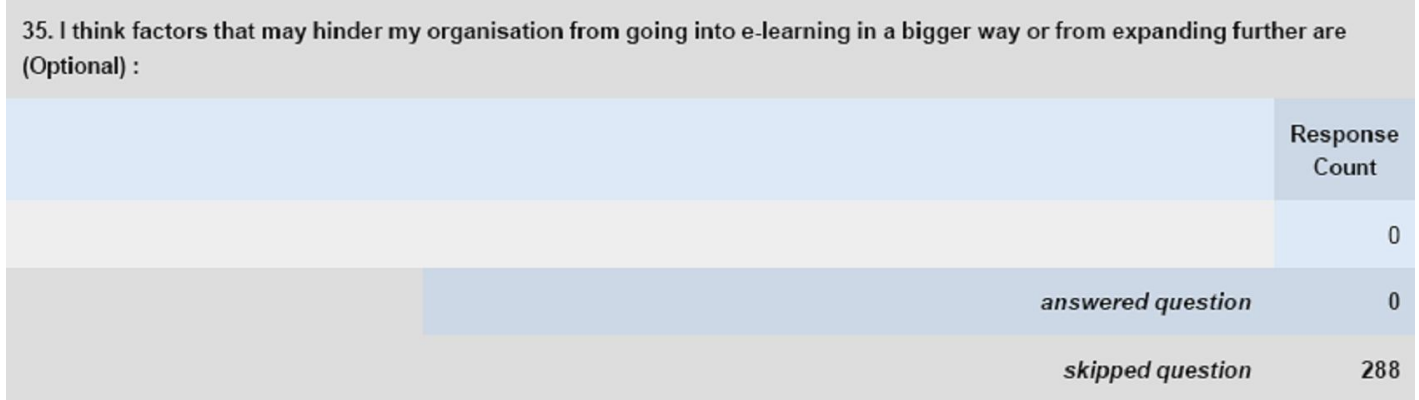

36. Other Comments - your other opinions, perceptions and suggestions relating to e-learning (Optional):

Response
Count
0
0
skipped question
answered question




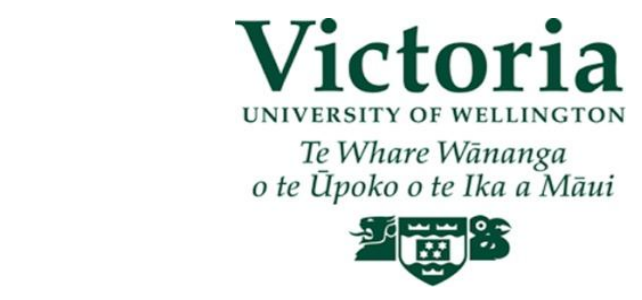

SIM HUMAN ETHICS COMMITTEE

Application for Approval of Research Projects

Please email applications to your supervisor, who will then email it to a SIM HEC member for a preliminary review.

Note: The Human Ethics Committee attempts to have all applications approved within 6 working days, but a longer period may be necessary if applications require substantial revision.

1 NATURE OF PROPOSED RESEARCH:

(a) Student Research

(b) If Student Research $\quad$ Degree MCA Course Code INFO591

(c) Project Title:

Measuring E-Learning Readiness across the Forestry Research and Development Agency (FORDA) of Indonesia

2 INVESTIGATORS:

(a) Principal Investigator

Name ..... Retisa Mutiaradevi (Student ID: 300101569).

e-mail address ... retisa.mutiaradevi@gmail.com

School/Dept/Group

School of Information Management

Faculty of Commerce \& Administration

(b) Other Researchers Name Position

None.

(c) Supervisor (in the case of student research projects)

Associate Professor Pak Yoong 
(a) Proposed starting date for data collection - After HEC approval has been granted.

(Note: that NO part of the research requiring ethical approval may commence prior to approval being given)

(b) Proposed date of completion of project as a whole ...11 February 2009...

\section{PROPOSED SOURCE/S OF FUNDING AND OTHER ETHICAL CONSIDERATIONS}

(a) Sources of funding for the project

Please indicate any ethical issues or conflicts of interest that may arise because of sources of funding

e.g. restrictions on publication of results

(b) Is any professional code of ethics to be followed

$\mathbf{N}$

If yes, name

(c) Is ethical approval required from any other body

If yes, name and indicate when/if approval will be given

None

\section{DETAILS OF PROJECT}

Briefly Outline:

(a) The objectives of the project

The research aims to investigate organisational readiness for implementing organisational e-learning systems (OES) in the Forestry Research and Development Agency (FORDA) Indonesia. Subsequently, it intends to determine factors that need to be addressed in order to implement successful OES in the FORDA. This study proposes a comprehensive organisational elearning readiness instrument (COERI) which can be used to assess all target respondents including policy makers, providers (e-learning providers/educational institutions), enablers (tutors, instructors, facilitators, trainers), and receivers (learners, trainees). The COERI is purposely designed to assess OE readiness within an organisation with no prior e-learning experience, specifically in a developing country in which OES are still relatively unknown.

(b) Method of data collection

The research will employ a mixed-method approach. Data collection will be conducted in two phases. Participation in either phase is voluntary for the participants.

In Phase 1, quantitative data will be collected from 20 FORDA branch offices by using a cross-sectional survey. The target population will be employees of FORDA offices. The size of the population is about 1,640. The sample frame will be a list of employees within 20 FORDA branch offices across the various regions. The study will use a stratified sampling design. The reporting unit will be individuals consisting FORDA employees, including managers (forestry directors, mid-level and low-level managers), researchers, research assistants, and general employees (including IT personnel). The sample size will be approximately half of the total number of employees in each office for 
each stratum. The level of observation will be each FORDA branch office and person/employee. The survey will be available to participants as either a paper-based survey or an online survey in order to maximise the number of people who can participate. The survey will be delivered in early August 2008, and are expected to be collected in late October 2008. A cover sheet that provides a brief research description, the directions, and introduction to the researcher will be added to the instrument.

In Phase 2, qualitative data will be collected through 8 or more semistructured interviews to complement the quantitative results. The interview respondents will be top level managers and/or top level IT personnel at FORDA. Interview participants can also participate in the survey.

The Secretary of FORDA has written letters of support which are addressed to all Forestry Directors in 20 FORDA offices across regions. The letters will be attached on the survey instrument and on the information sheet and participation consent form for the interview. There is a possibility for the researcher to meet all Forestry Directors in their annual meeting in early August. Therefore, there will be chances for handing over the survey kit to each branch office and conducting several interviews. The researcher will also visit several adjacent offices to conduct survey and interviews.

(c) The benefits and scientific value of the project

(1) Providing guidance for policy makers in the adoption of organisational elearning (OE) systems by recognising the level of organisation readiness towards OES;

(2) Providing a new system of organisational e-learning that can be implemented in the FORDA to improve organisational practices including providing training, sharing organisational knowledge and information, and promoting dissemination of the research results;

(3) Providing an initial internal assessment of OE readiness in the FORDA in order to develop successful OES in the future; and

(4) Enriching the existing body of knowledge in information systems field by providing a comprehensive organisational e-learning readiness instrument (COERI) and promoting 8 dimensions of OE readiness to measure the level of OE readiness in the developing countries context with no prior knowledge of e-learning.

(d) Characteristics of the participants

Participants for the survey will be FORDA employees across the regions (20 branch offices of FORDA in Indonesia) including managers, researchers, research assistants, and general employees (including IT personnel). Participants for the interview will be top level managers and/or top IT personnel (CIOs) at FORDA.

(e) Method of recruitment

The researcher is an employee of the FORDA and has been appointed by the organisation to be the leader of this research project. The researcher is able to make use of available resources in the FORDA including valuable information, research participants, and survey respondents.

The researcher has sought support from the main office, the Secretariat of FORDA, Jakarta. The Secretary of FORDA has provided a letter of support and permission for the researcher to conduct study in the FORDA across regions (the permission letter is attached to this document). The Secretary of FORDA also provided a letter addressed to each Forestry Director of FORDA to be attached to both the survey and the interview information sheets. 
Personal approach to interview participants will be made by sending confidential email followed by phone calls.

(f) Payments that are to be made/expenses to be reimbursed to participants

none.

(g) Other assistance (e.g. meals, transport) that is to be given to participants

none

(h) Any special hazards and/or inconvenience (including deception) that participants will encounter

No deception. Participants may be inconvenienced by giving their time for the survey and interviews.

(i) State whether consent is for: (Please indicate as many as it applies)
(i) the collection of data
$\mathbf{Y}$
(ii) attribution of opinions or information
$\mathbf{N}$
(iii) release of data to others
$\mathbf{N}$
(iv) use for a conference report or a publication $\mathbf{Y}$
(v) use for some particular purpose (specify) $\mathbf{Y}$

INFO591 - Information Systems Thesis

Attach a copy of any questionnaire or interview schedule to the application

(j) How is informed consent to be obtained (see paragraphs 4.31(g), 5.2, 5.5 and 5.61 of the Guidelines)

(i) the research is strictly anonymous, an information sheet is supplied and informed consent is implied by voluntary participation in filling out a questionnaire for example (include a copy of the information sheet)

(ii) the research is not anonymous but is confidential and informed consent will be obtained through a signed consent form (include a copy of the consent form and information sheet) $\quad \mathbf{Y}$

(iii) the research is neither anonymous nor confidential and informed consent will be obtained through a signed consent form (include a copy of the consent form and information sheet)

$\mathbf{N}$

(iv) informed consent will be obtained by some other method (please specify and provide details)

$\mathbf{N}$

With the exception of anonymous research as in (i), if it is proposed that written consent will not be obtained, please explain why 
(k)If the research will not be conducted on a strictly anonymous basis state how issues of confidentiality of participants are to be ensured if this is intended. (See paragraph 4.3.1(e) of the Guidelines). (e.g. who will listen to tapes, see questionnaires or have access to data). Please ensure that you distinguish clearly between anonymity and confidentiality. Indicate which of these are applicable.

(i) access to the research data will be restricted to the investigator

$\mathbf{N}$

(ii) access to the research data will be restricted to the investigator and their supervisor (student research) $\mathbf{Y}$

(iii) all opinions and data will be reported in aggregated form in such a way that individual persons or organisations are not identifiable

Y

(iv) Other (please specify)

(1) Procedure for the storage of, access to and disposal of data, both during and at the conclusion of the research. (see section 7 of the guidelines). Indicate which are applicable:

(i) all written material (questionnaires, interview notes, etc) will be kept in a locked file and access is restricted to the investigator $\mathbf{Y}$

(ii) all electronic information will be kept in a password-protected file and access will be restricted to the investigator Y

(iii) all questionnaires, interview notes and similar materials will be destroyed:

(a) at the conclusion of the research

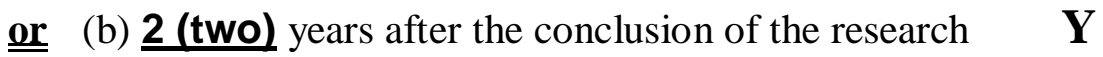

(iv) any audio or video recordings will be returned to participants and/or electronically wiped

$\mathbf{Y}$

(v) other procedures (please specify):

If data and material are not to be destroyed please indicate why and the procedures envisaged for ongoing storage and security

N/A

(m)Feedback procedures (See section 8 of the Guidelines). You should indicate whether feedback will be provided to participants and in what form. If feedback will not be given, indicate the reasons why.

The interview transcripts will be returned to each interview participant for checking of accuracy. A summary of the final research report will be available for all participants.

(n)Reporting and publication of results. Please indicate which of the following are appropriate. The proposed form of publications should be indicated on the information sheet and/or consent form. 
(i) publication in academic or professional journals

(ii) dissemination at academic or professional conferences

(iii) deposit of the research paper or thesis in the University Library (student research)

(iv) a case study used for teaching purposes

(v) other (please specify) 
Signature of investigators as listed on page 1 (including supervisors) and Chair of SIM HEC.

NB: All investigators and the Chair of SIM HEC must sign the form, then send it to the SIM HEC administrator for filing once the electronic application has been approved.

Date

Date.

Date.

Supervisors:

Date. ...

Date. . .

Chair of SIM HEC:

Date 


\section{APPLICATIONS FOR HUMAN ETHICS APPROVAL}

\section{CHECKLIST}

Have you read the Human Ethics Committee Policy?

Have you read the Faculty of Commerce and Administration's HEC Guide?

Is ethical approval required for your project?

Have you established whether informed consent needs to be obtained for your project?

In the case of student projects, have you consulted your supervisor about any human ethics implications of your research?

Have you included an information sheet for participants which explains the nature and purpose of your research, the proposed use of the material collected, who will have access to it, whether the data will be kept confidential to you, how anonymity or confidentiality is to be guaranteed?

Have you included a written consent form?

If not, have you explained on the application form why you do not need to get written consent?

Are you asking participants to give consent to:

$\square$ collect data from them

$\square$ attribute information to them

$\square$ release that information to others

$\square$ use the data for particular purposes

Have you indicated clearly to participants on the information sheet and/or consent form how they will be able to get feedback on the research from you (e.g. they may tick a box on the consent form indicating that they would like to be sent a summary), and how the data will be stored or disposed of at the conclusion of the research?

Have you included a copy of any questionnaire or interview checklist you propose using?

\section{POINTERS TO AVOID HAVING APPLICATIONS RETURNED BEFORE HEC REVIEW}

- The approval process is speeded up by not requiring the hard copy of your application form with the signatures on it at the initial review process. The complete application (HEC application form, info sheet, consent form, covering letter, questionnaire etc.) is to be emailed as an attachment in one file to your supervisor who will email it to an SIM HEC member for a preliminary review.

- Do not insert a date into item $3 \mathrm{a}$.

- Delete the "Y" or "N" option that is not required. DO NOT remove any other text from the application form.

- BOLD your answers if you wish but do not alter the font anywhere else in the form. 
Appendix 10: Information sheet - Survey

\author{
Victoria \\ UNIVERSITY OF WELLINGTON \\ Te Whare Wānanga \\ o te Ūpoko o te Ika a Māui \\ sq \\ SCHOOL OF INFORMATION MANAGEMENT \\ INFO591 -Information Systems Thesis \\ INFORMATION SHEET - Survey \\ Measuring E-Learning Readiness across \\ the Forestry Research and Development Agency (FORDA) of Indonesia
}

\begin{abstract}
About the Research
The research mainly focuses on assessing readiness towards organisational e-learning systems (OES) and identifying critical success factors for implementing successful OES in the FORDA. This study proposes a comprehensive organisational e-learning readiness instrument (COERI) which is purposely designed to assess OE readiness within an organisation with no prior e-learning experience specifically in an emerging country in which OES are still relatively unknown. The research is also intended to be undertaken in the completion of an MCA degree in Information Systems. The researcher is an employee of FORDA who is currently on a study leave for completing a Masters degree in Commerce and Administration (MCA) majoring in Information Systems at Victoria University of Wellington, New Zealand. Her previous duty was to organise the communication and dissemination of the research results in the Forestry Research Institute (FRI) of Surakarta.
\end{abstract}

The main benefits of this study are: (1) Providing guidance for policy makers to adopt OES by recognising the level of organisation readiness towards OES; (2) Introducing a new system of organisational e-learning that can be implemented in the FORDA to improve organisational practices including providing training, sharing organisational knowledge and information, and promoting dissemination of the research results; (3) Providing an initial internal assessment of OE readiness in the FORDA in order to develop successful OES in the future; and (4) Enriching the existing body of knowledge in information systems field by providing a comprehensive organisational e-learning readiness instrument (COERI) and promoting 8 dimensions of OE readiness to measure the level of OE readiness in the developing countries context with no prior knowledge of e-learning.

\title{
Confidentiality
}

- This questionnaire has been approved by Victoria University's Human Ethics Committee.

- All information collected will be considered confidential (no one except the researcher and the research supervisor will be permitted to see it).

- The participants will not be identified in the final report.

- Information collected will be kept in a locked filling cabinet and will be destroyed within two years after the completion of the research project.

- No copies will be made.

- No data will be passed to the employer.

- The results of this study might be published and disseminated as a conference report at academic or professional conferences or as a publication in academic or professional journals.

\section{Procedure}

Your participation is entirely voluntary. The questionnaire consists of three sections. The questionnaire will take approximately 30 minutes to complete, and can be completed either using the paper survey or online. By submitting the completed questionnaire, you are indicating your consent to participate in this research. If you have any queries about the research project, or your participation, please do not hesitate to contact me or my supervisor. The questionnaire is attached to this document.

Yours sincerely,

Ms. Retisa Mutiaradevi 


\title{
Appendix 11: Information sheet - Semi-structured interview
}

\author{
Victoria \\ UNIVERSITY OF WELLINGTON \\ Te Whare Wānanga \\ o te Ūpoko o te Ika a Māui

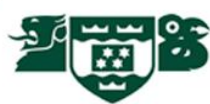 \\ SCHOOL OF INFORMATION MANAGEMENT \\ INFO591 -Information Systems Thesis \\ INFORMATION SHEET - Interview \\ Measuring E-Learning Readiness across \\ the Forestry Research and Development Agency (FORDA) of Indonesia
}

\begin{abstract}
About the Research
The research mainly focuses on assessing readiness towards organisational e-learning systems (OES) and identifying critical success factors for implementing successful OES in the FORDA. This study proposes a comprehensive organisational e-learning readiness instrument (COERI) which is purposely designed to assess OE readiness within an organisation with no prior e-learning experience specifically in an emerging country in which OES are still relatively unknown. The research is also intended to be undertaken in the completion of an MCA degree in Information Systems. The researcher is an employee of FORDA who is currently on a study leave for completing a Masters degree in Commerce and Administration (MCA) majoring in Information Systems at Victoria University of Wellington, New Zealand. Her previous duty was to organise the communication and dissemination of the research results in the Forestry Research Institute (FRI) of Surakarta.
\end{abstract}

The main benefits of this study are: (1) Providing guidance for policy makers to adopt organisational e-learning systems by recognising the level of organisation readiness towards OES; (2) Introducing a new system of organisational e-learning that can be implemented in the FORDA to improve organisational practices including providing training, sharing organisational knowledge and information, and promoting dissemination of the research results; (3) Providing an initial internal assessment of OE readiness in the FORDA in order to develop successful OES in the future; and (4) Enriching the existing body of knowledge in information systems field by providing a comprehensive organisational e-learning readiness instrument (COERI) and promoting 8 dimensions of OE readiness to measure the level of OE readiness in the developing countries context with no prior knowledge of e-learning.

\section{Confidentiality}

- This interview has been approved by Victoria University's Human Ethics Committee.

- All information collected will be considered confidential (no one except the researcher and the research supervisor will be permitted to see it).

- The participants will not be identified in the final report.

- Information collected will be kept in a locked filling cabinet and will be destroyed within two years after the completion of the research project.

- No copies will be made

- No data will be passed to the employer

- The results of this study might be published and disseminated as a conference report at academic or professional conferences or as a publication in academic or professional journals.

\section{Procedure}

The interview participation is entirely voluntary. The interview will last approximately 60 minutes and will be recorded by the interviewer using a recording device. The interview will be transcribed by the researcher and returned to the participant to be checked and validated for accuracy. You may withdraw from the project before 31 December 2008 without explanation, and any data have been provided will be returned to you or destroyed. The consent form is attached to this document. If you have any queries about the research project, or your participation, please do not hesitate to contact me or my supervisor.

Yours sincerely, Ms. Retisa Mutiaradevi 


\section{Victoria \\ UNIVERSITY OF WELLINGTON}

Te Whare Wānanga

o te Üpoko o te Ika a Māui

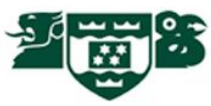

\section{SCHOOL OF INFORMATION MANAGEMENT \\ INF0591 -Information Systems Thesis \\ CONSENT TO PARTICIPATION IN RESEARCH \\ - Interview Participant -}

\section{Measuring E-Learning Readiness across \\ the Forestry Research and Development Agency (FORDA) of Indonesia}

Please tick ( $\square$ ) each box to indicate your agreement.

1. I I have been given and have understood the explanation of this research project. I have had an opportunity to ask questions and have them answered to my satisfaction.

2. I I understand that I may withdraw myself (or any information I have provided) from this project (before data collection and analysis is completed) without having to give reasons. Participation in this research project may be withdrawn before 31 December 2008 and any data I have provided will be returned to me or destroyed.

3. I understand that any information I provide will be kept confidential to the investigator (Retisa Mutiaradevi) and her research supervisor (Associate Professor Pak Yoong). The published results will not use my name, and no opinions will be attributed to me in any way that will identify me.

4. I I understand that the information I have provided will be used only for this research project and that any further use will require my written consent.

5. I understand that when this research is completed, the information obtained will be destroyed within two years after completion of the research project.

6. I agree to be interviewed by Retisa Mutiaradevi for the purpose of this research, and I consent to the collection and use of my perceptions, experiences, opinions, and information in this research.

7. I understand that the results of this study might be published and disseminated as a conference report at academic or professional conferences or as a publication in academic or professional journals

8. I would like a copy of the completed summary of the research results

9. I agree that the interview will be tape/video recorded

10. I confirm that I have approval of my employer to participate in this research project

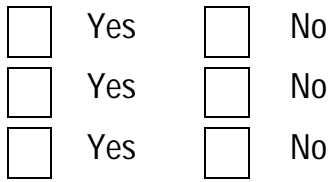

Name of Interview Participant

Signature of Interview Participant

$\frac{1}{\text { Date }}=\frac{12008}{2}$




\section{Comprehensive Organisational E-Learning Readiness Instrument (COERI)}

\section{SECTION A: Demographic}

A1. Employee ID Number

A2. Email address (if any)

A3. Gender:

$$
\square \text { Female } \quad \text { Male }
$$

A4. Age:

$$
\square \text { 18-25 } \square 26-30 \quad \square \text { 31-35 } \square 36-40 \quad \square 41-45 \quad \square 46-50 \quad \square 51-55 \quad \square>55
$$

A5. Level of education:

$$
\begin{array}{ll}
\square \text { High school } & \square \text { Postgraduate diploma } \\
\square \text { Certificate/Diploma } & \square \text { Master } \\
\square \text { Bachelors degree } & \\
\square \text { Other (please specify): } &
\end{array}
$$

A6. Which organisation in FORDA do you belong to?

$\square 1$ Secretariat of FORDA, JAKARTA

$\square 2$ Centre for Forest and Nature Conservation Research \& Development, BOGOR

\ 3 Centre for Forest Products Research \& Development, BOGOR

$\square 4$ Centre for Socio-Economic and Forestry Policy Research, BOGOR

$\square 5$ Centre for Plantation Forest Research and Development, BOGOR

$\square 6$ Centre for Dipterocarp Research, SAMARINDA

$\square 7$ Centre for Biotechnology and Forest Tree Improvement Research, YOGYAKARTA

$\square 8$ Forestry Research Institute of AEK NAUL

$\square 9$ Forestry Research Institute of MAKASSAR

$\square 10$ Forestry Research Institute of KUPANG

$\square 11$ Forestry Research Institute of MANOKWARI

$\square 12$ Forestry Research Institute of PALEMBANG

$\square 13$ Forestry Research Institute of BANJARBARU

$\square 14$ Forestry Research Institute of MATARAM

$\square 15$ Forestry Research Institute of MANADO

$\square 16$ Forestry Research Institute of SURAKARTA

$\square 17$ Forestry Research Institute of CIAMIS

$\square 18$ Forest Breeding Technology Research Institute of BOGOR

$\square$ 19 Forest Breeding Technology Research Institute of SAMBOJA

$\square 20$ Pulp and Fiber Forest Products Research Institute of KUOK

A7. Which business unit/section in your organisation do you belong to?
$\square$ Office of the Directorate
$\square$ Evaluation and Report
$\square$ Administration and General
$\square$ Program and Funding
$\square$ Planning and Funding
$\square$ Research Groups
$\square$ Services and Evaluation
$\square$ Research Facility and Resource
$\square$ Services and Dissemination
$\square$ Other (please specify):

A8. What is your job title?
$\square$ Director of the organisation
$\square$ Head of Section/Unit
$\square$ IT professional
$\square$ Researcher
$\square$ General Employee
$\square$ Research Assistant
$\square$ Other (please specify):

A9. Please provide a brief description of your job? 


\section{Section B: Prior e-learning knowledge}

B1. I have an Internet connection at my workplace:

$$
\square \text { Yes } \square \text { No }
$$

B2. I have an Internet connection at home:

$$
\square \text { Yes } \square \text { No }
$$

B3. I access the Internet mostly from:
$\square$ Home
$\square$ Wi-Fi Hot Spots
$\square$ Workplace
$\square$ Mobile phone/PDA
$\square$ Cyber/Internet café
$\square$ None
$\square$ College/university
$\square$ Other (please specify):

B4. Are you a member of any social networking sites?

$$
\square \text { No (skip to question B6) }
$$

$\square$ Yes, How many:

B5. Which social networking site do you use most frequently?

B6. Which online conference tools are you familiar with?
$\square$ Yahoo Messenger
$\square$ Google Chat
$\square$ MSN
$\square \mathrm{ICQ}$
$\square$ Skype
$\square$ Other, please specify:
$\square$ None

B7. Competencies for training in e-learning environments (tick the skill(s) that you have):
$\square$ Content expert
$\square$ Develop instructional media such as computer-assisted instruction (CAl)
$\square$ Graphic design
$\square$ Use instructional media such as computer-assisted instruction (CAI)
$\square$ Video production
$\square$ Develop instructional media such as slide shows
$\square$ Photography
$\square$ Use instructional media such as slide shows
$\square$ Storyboarding
$\square$ None
$\square$ Other, please specify:

B8. Please specify other computer programming skill(s) that you have:

B9. Please rank the following channels of communication for learning related to your work in FORDA. Please put a number from 1 (most preferred) to 6 (least preferred), note that two items cannot have the same rank order.

\begin{tabular}{|l|l|}
\hline $\begin{array}{l}\text { Rank } \\
\text { Number }\end{array}$ & $\begin{array}{l}\text { Communication } \\
\text { channels }\end{array}$ \\
\hline & Face-to-face \\
\hline & SMS \\
\hline & e-mail \\
\hline & chat on the Internet \\
\hline & written memo \\
\hline & postal mail \\
\hline
\end{tabular}




\section{Section C: E-learning readiness dimensions}

\section{Technological skills readiness}

\begin{tabular}{|l|l|c|c|c|c|c|}
\hline $\begin{array}{c}\text { Item } \\
\text { no }\end{array}$ & \multicolumn{1}{|c|}{ Questions } & $\begin{array}{c}\text { Very } \\
\text { proficient }\end{array}$ & Proficient & $\begin{array}{c}\text { Moderately } \\
\text { proficient }\end{array}$ & $\begin{array}{c}\text { Less } \\
\text { proficient }\end{array}$ & $\begin{array}{c}\text { Not at } \\
\text { all }\end{array}$ \\
\hline C.I.1 & $\begin{array}{l}\text { I have the basic skills to operate a computer (e.g. } \\
\text { keyboarding, using mouse, creating, saving, and } \\
\text { editing files, creating folders, managing file directories) }\end{array}$ & $\square 5$ & $\square 4$ & $\square 3$ & $\square 2$ & $\square 1$ \\
\hline C.I.2 & $\begin{array}{l}\text { I am good at using presentation packages (e.g. } \\
\text { PowerPoint), spreadsheets (e.g. Excel), and word } \\
\text { processor }\end{array}$ & $\square 5$ & $\square 4$ & $\square 3$ & $\square 2$ & $\square 1$ \\
\hline C.I.3 & $\begin{array}{l}\text { I know how to install software on my computer to } \\
\text { support my learning }\end{array}$ & $\square 5$ & $\square 4$ & $\square 3$ & $\square 2$ & $\square 1$ \\
\hline C.I.4 & $\begin{array}{l}\text { I have the basic skills for findings my way around the } \\
\text { Internet (e.g. using search engines to research } \\
\text { material, entering passwords) }\end{array}$ & $\square 5$ & $\square 4$ & $\square 3$ & $\square 2$ & $\square 1$ \\
\hline C.I.5 & $\begin{array}{l}\text { I know how to send and receive e-mail messages; and I } \\
\text { can send an email with a file attached }\end{array}$ & $\square 5$ & $\square 4$ & $\square 3$ & $\square 2$ & $\square 1$ \\
\hline C.I.6 & $\begin{array}{l}\text { I can troubleshoot most problems associated with using } \\
\text { a computer }\end{array}$ & $\square 5$ & $\square 4$ & $\square 3$ & $\square 2$ & $\square 1$ \\
\hline C.1.7 & $\begin{array}{l}\text { I feel that prior experiences with online technologies } \\
\text { (e.g. email, Internet chat, online readings) are important } \\
\text { to my success with online course }\end{array}$ & $\square 5$ & $\square 4$ & $\square 3$ & $\square 2$ & $\square 1$ \\
\hline
\end{tabular}

\section{Online learning style readiness}

\begin{tabular}{|l|l|c|c|c|c|c|}
\hline $\begin{array}{c}\text { Item } \\
\text { no }\end{array}$ & \multicolumn{1}{|c|}{ Questions } & $\begin{array}{c}\text { Strongly } \\
\text { agree }\end{array}$ & Agree & Neutral & Disagree & $\begin{array}{c}\text { Strongly } \\
\text { disagree }\end{array}$ \\
\hline C.II.1 & $\begin{array}{l}\text { I think I would be able to read and learn, or follow the } \\
\text { direction on a computer screen to accomplish a task }\end{array}$ & $\square 5$ & $\square 4$ & $\square 3$ & $\square 2$ & $\square 1$ \\
\hline C.II.2 & $\begin{array}{l}\text { I think that I would be able to take notes while watching a } \\
\text { video on the computer and I think that would be able to } \\
\text { relate the content of short video clips (1-3 minutes } \\
\text { typically) to the information I have read online or in books }\end{array}$ & $\square 5$ & $\square 4$ & $\square 3$ & $\square 2$ & $\square 1$ \\
\hline C.II.3 & $\begin{array}{l}\text { I think that I would be comfortable having several } \\
\text { discussions taking place in the same online chat even } \\
\text { though I may not be participating in all of them }\end{array}$ & $\square 5$ & $\square 4$ & $\square 3$ & $\square 2$ & $\square 1$ \\
\hline C.II.4 & $\begin{array}{l}\text { I think that I would be able to use online tools (e.g. email, } \\
\text { chat) to work on assignments with learners who are in } \\
\text { different time zones }\end{array}$ & $\square 5$ & $\square 4$ & $\square 3$ & $\square 2$ & $\square 1$ \\
\hline C.II.5 & $\begin{array}{l}\text { I would rather listen to a lecture than read the material } \\
\text { from a computer screen }\end{array}$ & $\square 5$ & $\square 4$ & $\square 3$ & $\square 2$ & $\square 1$ \\
\hline C.II.6 & $\begin{array}{l}\text { I would rather find out information using a computer than } \\
\text { from a teacher or lecturer }\end{array}$ & $\square 5$ & $\square 4$ & $\square 3$ & $\square 2$ & $\square 1$ \\
\hline C.II.7 & $\begin{array}{l}\text { I cannot learn using only computers, I need the } \\
\text { teacher/student contact }\end{array}$ & $\square 5$ & $\square 4$ & $\square 3$ & $\square 2$ & $\square 1$ \\
\hline C.II.8 & $\begin{array}{l}\text { I think regular contact with the instructor is important to } \\
\text { my success in online coursework }\end{array}$ & $\square 5$ & $\square 4$ & $\square 3$ & $\square 2$ & $\square 1$ \\
\hline C.II.9 & $\begin{array}{l}\text { I think frequent participation throughout the learning } \\
\text { process is important to my success in online coursework }\end{array}$ & $\square 5$ & $\square 4$ & $\square 3$ & $\square 2$ & $\square 1$ \\
\hline
\end{tabular}




\section{Equipment/infrastructure readiness}

\begin{tabular}{|l|l|c|c|c|c|c|}
\hline $\begin{array}{c}\text { Item } \\
\text { no }\end{array}$ & \multicolumn{1}{|c|}{ Questions } & $\begin{array}{c}\text { Almost } \\
\text { everyday }\end{array}$ & $\begin{array}{c}\text { More } \\
\text { than } \\
\text { once a } \\
\text { week }\end{array}$ & $\begin{array}{c}\text { Once a } \\
\text { week }\end{array}$ & Rarely & Never \\
\hline C.III.1 & $\begin{array}{l}\text { I have access to a computer and can use it individually } \\
\text { at work }\end{array}$ & $\square 5$ & $\square 4$ & $\square 3$ & $\square 2$ & $\square 1$ \\
\hline C.III.2 & $\begin{array}{l}\text { I have access to a fairly new computer (e.g. enough } \\
\text { RAM, speakers, CD-ROM) }\end{array}$ & $\square 5$ & $\square 4$ & $\square 3$ & $\square 2$ & $\square 1$ \\
\hline C.III.3 & $\begin{array}{l}\text { I have access to a computer with adequate software } \\
\text { (e.g. Microsoft Word, Adobe Acrobat) }\end{array}$ & $\square 5$ & $\square 4$ & $\square 3$ & $\square 2$ & $\square 1$ \\
\hline C.III.4 & $\begin{array}{l}\text { My workplace is using Internet technology to run its } \\
\text { daily operations }\end{array}$ & $\square 5$ & $\square 4$ & $\square 3$ & $\square 2$ & $\square 1$ \\
\hline C.III.5 & $\begin{array}{l}\text { My workplace is using Intranet technology to run its } \\
\text { daily operations }\end{array}$ & $\square 5$ & $\square 4$ & $\square 3$ & $\square 2$ & $\square 1$ \\
\hline C.III.6 & $\begin{array}{l}\text { I think poor infrastructure may hinder my organisation } \\
\text { from developing and implementing into e-learning }\end{array}$ & $\square 5$ & $\square 4$ & $\square 3$ & $\square 2$ & $\square 1$ \\
\hline C.III.7 & $\begin{array}{l}\text { I think lack of content may hinder my organisation from } \\
\text { developing and implementing into e-learning }\end{array}$ & $\square 5$ & $\square 4$ & $\square 3$ & $\square 2$ & $\square 1$ \\
\hline C.III.8 & $\begin{array}{l}\text { I think quick technical and administrative support is } \\
\text { important to my success in online coursework }\end{array}$ & $\square 5$ & $\square 4$ & $\square 3$ & $\square 2$ & $\square 1$ \\
\hline
\end{tabular}

\section{Attitude readiness}

\begin{tabular}{|c|c|c|c|c|c|c|}
\hline \multicolumn{2}{|c|}{ Confidence } & \multirow{2}{*}{$\begin{array}{c}\text { Strongly } \\
\text { agree }\end{array}$} & \multirow{2}{*}{$\begin{array}{c}\text { Agree } \\
\square 4\end{array}$} & \multirow{2}{*}{$\begin{array}{c}\text { Neutral } \\
\square 3\end{array}$} & \multirow{2}{*}{$\begin{array}{c}\text { Disagree } \\
\square 2\end{array}$} & \multirow{2}{*}{$\begin{array}{c}\text { Strongly } \\
\text { disagree } \\
\square 1\end{array}$} \\
\hline C.IV.1 & $\begin{array}{l}\text { I feel confidence in my ability to use advanced } \\
\text { technology in training and/or learning }\end{array}$ & & & & & \\
\hline C.IV.2 & I hesitate to use technology for fear of making mistakes & $\square 5$ & $\square 4$ & $\square 3$ & $\square 2$ & $\square 1$ \\
\hline C.IV.3 & $\begin{array}{l}\text { I can teach myself most of the things I need to know } \\
\text { about using e-learning technologies }\end{array}$ & $\square 5$ & $\square 4$ & $\square 3$ & $\square 2$ & $\square 1$ \\
\hline C.IV.4 & $\begin{array}{l}\text { I would feel better about using technology if I knew } \\
\text { more about them }\end{array}$ & $\square 5$ & $\square 4$ & $\square 3$ & $\square 2$ & $\square 1$ \\
\hline C.IV.5 & $\begin{array}{l}\text { I feel threatened when I see others using technology in } \\
\text { their learning/working }\end{array}$ & $\square 5$ & $\square 4$ & $\square 3$ & $\square 2$ & $\square 1$ \\
\hline C.IV.6 & $\begin{array}{l}\text { Knowing how to use e-learning technologies will } \\
\text { increase my job possibilities }\end{array}$ & $\square 5$ & $\square 4$ & $\square 3$ & $\square 2$ & $\square 1$ \\
\hline \multicolumn{2}{|c|}{ Enjoyment } & $\begin{array}{l}\text { Strongly } \\
\text { agree }\end{array}$ & Agree & Neutral & Disagree & $\begin{array}{l}\text { Strongly } \\
\text { disagree }\end{array}$ \\
\hline C.IV.7 & I like using computers for research & $\square 5$ & $\square 4$ & $\square 3$ & $\square 2$ & $\square 1$ \\
\hline C.IV.8 & $\begin{array}{l}\text { I like to communicate with others using e-mail to } \\
\text { support my learning }\end{array}$ & $\square 5$ & $\square 4$ & $\square 3$ & $\square 2$ & $\square 1$ \\
\hline C.IV.9 & I spend a lot of time on the Internet & $\square 5$ & $\square 4$ & $\square 3$ & $\square 2$ & $\square 1$ \\
\hline C.IV.10 & $\begin{array}{l}\text { I enjoy working on tasks on a computer that I can do by } \\
\text { following directions }\end{array}$ & $\square 5$ & $\square 4$ & $\square 3$ & $\square 2$ & $\square 1$ \\
\hline C.IV.11 & $\begin{array}{l}\text { I like to try new technologies for teaching, training } \\
\text { and/or learning }\end{array}$ & $\square 5$ & $\square 4$ & $\square 3$ & $\square 2$ & $\square 1$ \\
\hline \multicolumn{2}{|c|}{ Importance } & $\begin{array}{l}\text { Strongly } \\
\text { agree }\end{array}$ & Agree & Neutral & Disagree & $\begin{array}{l}\text { Strongly } \\
\text { disagree }\end{array}$ \\
\hline C.IV.12 & $\begin{array}{l}\text { It is important to learn how to use a computer for } \\
\text { learning and training, and how to use the Internet as a } \\
\text { learning resource }\end{array}$ & $\square 5$ & $\square 4$ & $\square 3$ & $\square 2$ & $\square 1$ \\
\hline C.IV.13 & $\begin{array}{l}\text { Learning about how to use e-learning technologies is a } \\
\text { waste of time }\end{array}$ & $\square 5$ & $\square 4$ & $\square 3$ & $\square 2$ & $\square 1$ \\
\hline \multicolumn{2}{|c|}{ Motivation } & $\begin{array}{l}\text { Strongly } \\
\text { agree }\end{array}$ & Agree & Neutral & Disagree & $\begin{array}{l}\text { Strongly } \\
\text { disagree }\end{array}$ \\
\hline C.IV.14 & $\begin{array}{l}\text { I think that I would be able to remain motivated even } \\
\text { though the instructor is not online at all times }\end{array}$ & $\square 5$ & $\square 4$ & $\square 3$ & $\square 2$ & $\square 1$ \\
\hline C.IV.15 & $\begin{array}{l}\text { I think that I would be able to complete my work even } \\
\text { when there are online distractions (e.g. friends sending } \\
\text { emails or Websites to surf) }\end{array}$ & $\square 5$ & $\square 4$ & $\square 3$ & $\square 2$ & $\square 1$ \\
\hline C.IV.16 & $\begin{array}{l}\text { I think that I would be able to complete my work even } \\
\text { when there are distractions in my home (e.g. television, } \\
\text { children, and such) }\end{array}$ & $\square 5$ & $\square 4$ & $\square 3$ & $\square 2$ & 口1 \\
\hline
\end{tabular}




\begin{tabular}{|c|c|c|c|c|c|c|}
\hline \multicolumn{2}{|c|}{ Self-development } & \multirow{2}{*}{$\begin{array}{c}\text { Strongly } \\
\text { agree }\end{array}$} & \multirow{2}{*}{$\frac{\text { Agree }}{\square 4}$} & \multirow{2}{*}{$\begin{array}{c}\text { Neutral } \\
3\end{array}$} & \multirow{2}{*}{$\begin{array}{c}\text { Disagree } \\
\square 2\end{array}$} & \multirow{2}{*}{$\begin{array}{c}\begin{array}{c}\text { Strongly } \\
\text { disagree }\end{array} \\
\square 1\end{array}$} \\
\hline C.IV.17 & $\begin{array}{l}\text { I think I can spend some time }(15,30 \text {, or } 60 \text { minutes) } \\
\text { for improving myself during some part of the day } \\
\text { (morning, afternoon, evening, or night) }\end{array}$ & & & & & \\
\hline C.IV.18 & $\begin{array}{l}\text { I believe that self-development of employees may } \\
\text { strengthen the position of the unit in the organisation }\end{array}$ & $\square 5$ & $\square 4$ & $\square 3$ & $\square 2$ & 口1 \\
\hline C.IV.19 & $\begin{array}{l}\text { I am interested to upgrade my academic/professional } \\
\text { qualification and/or work performance through e- } \\
\text { learning }\end{array}$ & $\square 5$ & $\square 4$ & $\square 3$ & $\square 2$ & $\square 1$ \\
\hline \multicolumn{2}{|r|}{ 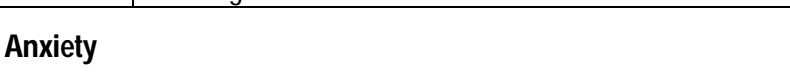 } & $\begin{array}{l}\text { Strongly } \\
\text { agree }\end{array}$ & Agree & Neutral & Disagree & $\begin{array}{l}\text { Strongly } \\
\text { disagree }\end{array}$ \\
\hline C.IV.20 & $\begin{array}{l}\text { I think positively toward the technological interventions } \\
\text { in daily/routine tasks }\end{array}$ & $\square 5$ & $\square 4$ & $\square 3$ & $\square 2$ & $\square 1$ \\
\hline C.IV.21 & $\begin{array}{l}\text { I feel comfortable with the thought of using technology } \\
\text { to deliver instruction }\end{array}$ & $\square 5$ & $\square 4$ & $\square 3$ & $\square 2$ & $\square 1$ \\
\hline C.IV.22 & $\begin{array}{l}\text { The thought of using e-learning technologies makes } \\
\text { me very nervous }\end{array}$ & $\square 5$ & $\square 4$ & $\square 3$ & $\square 2$ & 口1 \\
\hline C.IV.23 & $\begin{array}{l}\text { I get a sinking feeling when I think of trying to use } \\
\text { technology for training trainees and/or learning }\end{array}$ & $\square 5$ & $\square 4$ & $\square 3$ & $\square 2$ & 口1 \\
\hline
\end{tabular}

\section{Human resource readiness}

\begin{tabular}{|c|c|c|c|c|c|c|}
\hline \multicolumn{2}{|c|}{ Management } & \multirow{2}{*}{$\begin{array}{c}\text { Strongly } \\
\text { agree }\end{array}$} & \multirow{2}{*}{$\begin{array}{c}\text { Agree } \\
\square 4 \\
\end{array}$} & \multirow{2}{*}{$\begin{array}{c}\text { Neutral } \\
\square 3 \\
\end{array}$} & \multirow{2}{*}{$\begin{array}{c}\text { Disagree } \\
\square 2 \\
\end{array}$} & \multirow{2}{*}{$\begin{array}{c}\begin{array}{c}\text { Strongly } \\
\text { disagree }\end{array} \\
\square 1\end{array}$} \\
\hline C.V.1 & My workplace has a vision/mission on e-learning & & & & & \\
\hline C.V.2 & $\begin{array}{l}\text { The high and mid level managers think positively } \\
\text { toward the technological interventions in daily/routine } \\
\text { tasks }\end{array}$ & $\square 5$ & $\square 4$ & $\square 3$ & $\square 2$ & $\square 1$ \\
\hline C.V.3 & $\begin{array}{l}\text { The majority of high and mid level managers have } \\
\text { accepted any changes that required the use of } \\
\text { technology in daily/routine tasks }\end{array}$ & $\square 5$ & $\square 4$ & $\square 3$ & $\square 2$ & $\square 1$ \\
\hline C.V.4 & $\begin{array}{l}\text { My employer will give me the time off to study via e- } \\
\text { learning and will let me use the facilities at work } \\
\text { outside office hours for e-learning }\end{array}$ & $\square 5$ & $\square 4$ & $\square 3$ & $\square 2$ & $\square 1$ \\
\hline \multicolumn{2}{|c|}{ Personnel } & $\begin{array}{c}\text { Very } \\
\text { proficient }\end{array}$ & Proficient & $\begin{array}{l}\text { Moderately } \\
\text { proficient }\end{array}$ & $\begin{array}{c}\text { Less } \\
\text { proficient }\end{array}$ & Not at all \\
\hline C.V.5 & $\begin{array}{l}\text { I am experienced to organise and evaluate training } \\
\text { and to help other employees with career development }\end{array}$ & $\square 5$ & $\square 4$ & $\square 3$ & $\square 2$ & $\square 1$ \\
\hline C.V.6 & $\begin{array}{l}\text { I am the person who can facilitate the acceptance and } \\
\text { implementation of e-learning initiative in my workplace }\end{array}$ & $\square 5$ & $\square 4$ & $\square 3$ & $\square 2$ & $\square 1$ \\
\hline C.V.7 & $\begin{array}{l}\text { I have experience with technology-based or assisted } \\
\text { training (e.g. computer-based training, multimedia- } \\
\text { based learning, video cassettes, etc) }\end{array}$ & $\square 5$ & $\square 4$ & $\square 3$ & $\square 2$ & $\square 1$ \\
\hline C.V.8 & I am able to design Web pages for e-learning & $\square 5$ & $\square 4$ & $\square 3$ & $\square 2$ & $\square 1$ \\
\hline C.V.9 & I am able to moderate online discussions & $\square 5$ & $\square 4$ & $\square 3$ & $\square 2$ & $\square 1$ \\
\hline C.V.10 & $\begin{array}{l}\text { I am able to write good study guides for e-learning } \\
\text { students }\end{array}$ & $\square 5$ & $\square 4$ & $\square 3$ & $\square 2$ & $\square 1$ \\
\hline C.V.11 & $\begin{array}{l}\text { I am able to deal with legal issues related to e-learning } \\
\text { (copyrights, privacy) }\end{array}$ & $\square 5$ & $\square 4$ & $\square 3$ & $\square 2$ & $\square 1$ \\
\hline
\end{tabular}

\section{Environmental readiness}

\begin{tabular}{|l|l|c|c|c|c|c|}
\hline \multicolumn{1}{|c|}{ Item no } & \multicolumn{1}{|c|}{ Questions } & $\begin{array}{c}\text { Strongly } \\
\text { agree }\end{array}$ & Agree & Neutral & Disagree & $\begin{array}{c}\text { Strongly } \\
\text { disagree }\end{array}$ \\
\hline C.VI.1 & $\begin{array}{l}\text { Organisational policies have made my } \\
\text { organisation/institution keen to explore e-learning }\end{array}$ & $\square 5$ & $\square 4$ & $\square 3$ & $\square 2$ & $\square 1$ \\
\hline C.VI.2 & $\begin{array}{l}\text { The lack of legal provision on intellectual property has } \\
\text { hindered the development of e-learning in my } \\
\text { organisation/institution }\end{array}$ & $\square 5$ & $\square 4$ & $\square 3$ & $\square 2$ & $\square 1$ \\
\hline C.VI.3 & $\begin{array}{l}\text { Certain organisational policies have hindered our } \\
\text { plans to invest in e-learning }\end{array}$ & $\square 5$ & $\square 4$ & $\square 3$ & $\square 2$ & $\square 1$ \\
\hline C.VI.4 & $\begin{array}{l}\text { It is important to have a central agency to play an } \\
\text { active role in regulating, competency development, } \\
\text { research, intelligence gathering and e-learning } \\
\text { initiatives in the FORDA }\end{array}$ & $\square 5$ & $\square 4$ & $\square 3$ & $\square 2$ & $\square 1$ \\
\hline
\end{tabular}




\section{Cultural readiness}

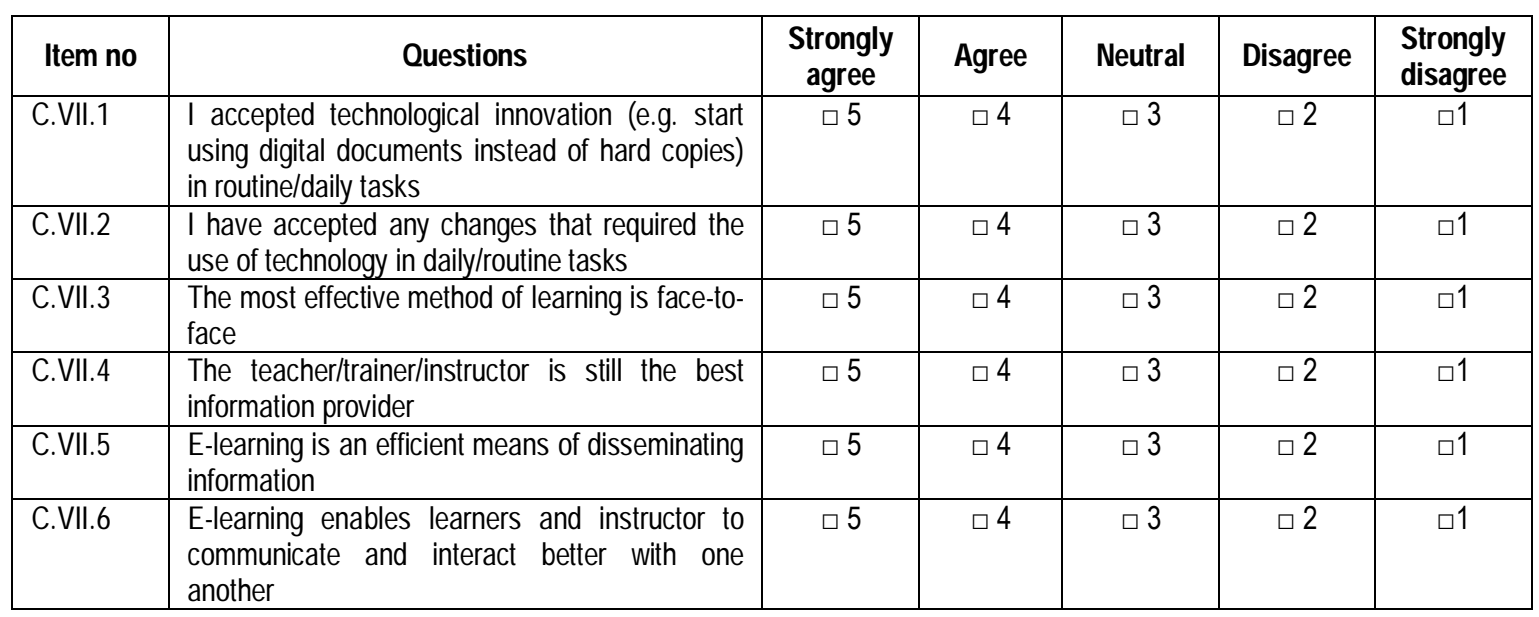

\section{Financial readiness}

\begin{tabular}{|c|c|c|c|c|c|c|}
\hline $\begin{array}{c}\text { Item } \\
\text { no }\end{array}$ & Questions & $\begin{array}{l}\text { Very } \\
\text { feasible }\end{array}$ & Feasible & $\begin{array}{l}\text { Somewhat } \\
\text { feasible }\end{array}$ & $\begin{array}{l}\text { Less } \\
\text { feasible }\end{array}$ & Not at all \\
\hline C.VIII.1 & $\begin{array}{l}\text { My organisation/institution provides a computer } \\
\text { loan to the employees }\end{array}$ & $\square 5$ & $\square 4$ & $\square 3$ & $\square 2$ & $\begin{array}{ll}1 \\
\end{array}$ \\
\hline C.VIII.2 & $\begin{array}{l}\text { I can take a loan to buy a computer for e- } \\
\text { learning purposes }\end{array}$ & $\square 5$ & $\square 4$ & $\square 3$ & $\square 2$ & $\square 1$ \\
\hline C.VIII.3 & $\begin{array}{l}\text { I can afford to buy a computer and pay for } \\
\text { Internet access }\end{array}$ & $\square 5$ & $\square 4$ & $\square 3$ & $\square 2$ & 口1 \\
\hline \multirow[t]{2}{*}{ C.VIII.4 } & $\begin{array}{l}\text { My organisation/institution provides funds for } \\
\text { employees to attend conferences and training }\end{array}$ & $\square 5$ & $\square 4$ & $\square 3$ & $\square 2$ & $\begin{array}{ll}1 \\
\end{array}$ \\
\hline & & $\begin{array}{l}\text { Strongly } \\
\text { agree }\end{array}$ & Agree & Neutral & Disagree & $\begin{array}{l}\text { Strongly } \\
\text { disagree }\end{array}$ \\
\hline C.VIII.5 & $\begin{array}{l}\text { I think, my organisation/institution can create a } \\
\text { budget for implementing e-learning }\end{array}$ & $\square 5$ & $\square 4$ & $\square 3$ & $\square 2$ & $\square 1$ \\
\hline C.VIII.6 & $\begin{array}{l}\text { I think high initial investment may hinder my } \\
\text { organisation from developing and implementing } \\
\text { into e-learning }\end{array}$ & $\square 5$ & $\square 4$ & $\square 3$ & $\square 2$ & $\square 1$ \\
\hline C.VIII.7 & $\begin{array}{l}\text { I think high operating costs may hinder my } \\
\text { organisation from developing and implementing } \\
\text { into e-learning }\end{array}$ & $\square 5$ & $\square 4$ & $\square 3$ & $\square 2$ & $\square 1$ \\
\hline
\end{tabular}

I think factors that may hinder my organisation from going into e-learning in a bigger way or from expanding further are (Optional) :

Other Comments - your other opinions, perceptions and suggestions relating to e-learning (Optional) : 


\section{Appendix 13: Interview questions}

\begin{tabular}{|c|c|}
\hline No & Questions \\
\hline \multirow[t]{4}{*}{1.} & $\begin{array}{l}\text { What do you think about organisational e-learning (OE) being implemented in the } \\
\text { organisation? }\end{array}$ \\
\hline & a) What kind of training have you done in the past 3 years? Has it changed? How? \\
\hline & $\begin{array}{l}\text { b) Have you done any organisational e-learning of flexible e-learning in your organisation } \\
\text { before? Are you planning to do any in the future? (E.g. who are the users, who are the } \\
\text { providers, and what is the range of courses that will be covered through the } \\
\text { organisational e-learning?) }\end{array}$ \\
\hline & $\begin{array}{l}\text { c) Do you see a clear strategic advantage for using organisational e-learning solutions in } \\
\text { the organisation? (e.g. Do you think the use of learning technologies can raise the } \\
\text { standards of an employee's performance?) }\end{array}$ \\
\hline 2. & $\begin{array}{l}\text { In the past employees have been provided with incentives to participate in training / } \\
\text { seminars / etc. Are there any current plans to provide employees with incentives to } \\
\text { participate in organisational e-learning? What kind of incentives? What would qualify people } \\
\text { to receive the incentives? }\end{array}$ \\
\hline \multirow[t]{3}{*}{3.} & $\begin{array}{l}\text { What are the potential challenges that the organisation may face in the setting-up and/or } \\
\text { implementing flexible e-learning? }\end{array}$ \\
\hline & $\begin{array}{l}\text { a) What do you think will be the top } 3 \text { barriers to starting up or implementing } \\
\text { organisational e-learning? }\end{array}$ \\
\hline & $\begin{array}{l}\text { b) What barriers do you think may slow down employees' adoption of organisational e- } \\
\text { learning? }\end{array}$ \\
\hline \multirow[t]{3}{*}{4.} & $\begin{array}{l}\text { What level of investment do you think the organisation has made including IT infrastructure } \\
\text { and human resources in order to support organisational e-learning? }\end{array}$ \\
\hline & a) Do you think organisational e-learning is worth the investment? Why? \\
\hline & $\begin{array}{l}\text { b) Do you believe you will see a return on your investment into organisational e-learning? } \\
\text { What return? (e.g. financial, time, productivity, etc) }\end{array}$ \\
\hline \multirow[t]{4}{*}{5.} & $\begin{array}{l}\text { In your opinion, how ready do you think the organisation is to implement organisational e- } \\
\text { learning? }\end{array}$ \\
\hline & $\begin{array}{l}\text { a) Do you think the existing network technology supports FORDA personnel interacting } \\
\text { with each other in a flexible e-learning environment? Explain? }\end{array}$ \\
\hline & $\begin{array}{l}\text { b) Do you think FORDA personnel are eager and/or well-motivated to get together in an } \\
\text { online, e-learning environment? }\end{array}$ \\
\hline & $\begin{array}{l}\text { c) How well do you think the training policy supports flexible e-lea } \\
\text { provide an example?) }\end{array}$ \\
\hline
\end{tabular}

